JORDI MAS SOLER

ASSESSING THE USE OF A SEMISUBMERSIBLE

OIL PLATFORM AS A MOTION-BASED SEA WAVE SENSOR 
JORDI MAS SOLER

\section{ASSESSING THE USE OF A SEMISUBMERSIBLE OIL PLATFORM AS A MOTION-BASED SEA WAVE SENSOR}

Thesis submitted to the Escola Politécnica da Universidade de São Paulo for the partial fulfillment of the requirements for the degree of Doctor of Science. 


\section{ASSESSING THE USE OF A SEMISUBMERSIBLE OIL PLATFORM AS A MOTION-BASED SEA WAVE SENSOR}

Thesis submitted to the Escola Politécnica da Universidade de São Paulo for the partial fulfillment of the requirements for the degree of Doctor of Science.

Research Area:

Naval Architecture and Ocean Engineering

Surpervisor:

Prof. Alexandre Nicolaos Simos

Co-Supervisor:

Prof. Antonio Souto Iglesias 
Autorizo a reprodução e divulgação total ou parcial deste trabalho, por qualquer meio convencional ou eletrônico, para fins de estudo e pesquisa, desde que citada a fonte.

\section{Catalogação-na-publicação}

\section{MAS SOLER, JORDI}

Assessing the use of a semisubmersible oil platform as a motion-based sea wave sensor / J. MAS SOLER -- São Paulo, 2018.

$227 \mathrm{p}$.

Tese (Doutorado) - Escola Politécnica da Universidade de São Paulo. Departamento de Engenharia Naval e Oceânica.

1.Semisubmersible Platform 2.Viscous Damping 3.Motion-Based Wave Inference 4.Bayesian Inference 5.Prior Distribution I.Universidade de São Paulo. Escola Politécnica. Departamento de Engenharia Naval e Oceânica II.t. 


\section{ACKNOWLEDGMENTS}

First and foremost, I would like to express my deepest and sincere gratitude to my advisor prof. Alexandre Nicolaos Simos, without him the majority of experiences I have had during the last years could have never been possible. I really appreciate his commitment and advice, keeping me always confident about my work. Also, I am thankful for his expertise, understanding, patience, professionalism and wisdom, that outstandingly contributed to my personal and professional progress.

I am also, and I will ever be, indebted to my co-advisor prof. Antonio Souto Iglesias. He has taught me, both consciously and unconsciously, how good research is done. I also appreciate all his contributions of time, ideas and the professional guidance and support along all these years.

I gratefully acknowledge the funding sources that made this work possible. I was financed by the Coordenação de Aperfeiçoamento de Pessoal de Nível Superior - Brasil (CAPES) - Finance Code 001. Also, I would like to express my most sincerely thanks and gratitude to EQUINOR (ex-STATOIL), Petrobras and the Brazilian Petroleum Agency (ANP) for making the experimental campaign possible and providing all the technical resources necessary for this research.

A special thanks go to my colleagues at Numerical Offshore Tank in the University of São Paulo (TPN-USP): Iuri Bispo, Asdrubal Nascimento, Thiago Hallak, prof. Eduardo Tannuri, prof. Guilherme Franzini, prof. André Fujarra and prof. Pedro de Mello for the many discussions and experiences in the last years. I am particularly grateful to my friend Felipe Lopes. We shared several good moments and thanks to him I have grow up significantly. Also, his direct and indirect contributions to this work are unmeasurable.

There is an endless list of people that I had the opportunity to meet during the these years, and that have been of utmost importance to me, each one in a different and significant way. However, I would like to thank my friends at the TPN-USP for making my stay at the university more than just studying and working on my research. In particular, I am grateful to Daniel Kantorowitz, Kam Yu Kang, Lariuss Zago, Fernanda Hille and Lucas Carmo for making my days more interesting, enjoyable and funny.

Last but not the least, special gratitude and love to my caring family, Cèlia, Hèctor, Reme and Alberto, for supporting and motivating me in all my pursuits. They are always in my heart, even if I do not phone them as much as I should. And most of all for my loving Karla, for making me a better person and for whose faithful support and encouragement during all the stages of this work, that are so appreciated and invaluable. Thank you. 
"Probability is orderly opinion and inference from data is nothing other than the revision of such opinion in the light of relevant new information"

-Robert Schlaifer. Probability and Statistics for Business Decisions 


\section{ABSTRACT}

This thesis assesses the use of the measured motions of a semisubmersible oil platform as a basis for estimating on-site wave spectra. The inference method followed is based on the wave buoy analogy, which aims at solving the linear inverse problem: estimate the sea state, given the measured motions and the transfer function of the platform.

Directional wave inference obtained from the records of vessels motions is a technique that has seen its application grow significantly over the last years. As a matter of fact, its applications in ships with forward speed and ship-shaped moored platforms (such as FPSOs) have provided good results. However, little research has been done regarding the use of semisubmersible platforms as wave sensors. This is due to the fact that these platforms are designed to present no significant responses when excited by waves. Notwithstanding this, the semisubmersible platforms are characterized by measurable small motions. Moreover, if compared with ship-shaped motion-based wave sensors, the responses of the semisubmersibles are in better agreement with the response characteristics estimations obtained by means of linear hydrodynamic models. In addition, the eminently linear characteristics of the responses often lasts even for severe wave conditions. This feature results in that the semisubmersible platforms stand as a promising wave sensor even for extreme sea states, conditions in which other types of sensors (i.e. buoys, radars) may face difficulties. Throughout the text, the main results of this work are presented and discussed. These results are mainly based on a dedicated experimental campaign, carried out with a scaled model of the Åsgar-B platform, which is a semisubmersible platform located in the Åsgard field offshore Norway. Regarding the sea states tested during the experiential campaign, they were estimated by means of a motion-based Bayesian inference method, which has been developed for more than then years at the EPUSP. In order to allow the adoption of the semisubmersible platforms as a motion based wave sensors, this thesis provides two significant improvements of the method: first, a method to obtain an estimation of the linearized equivalent external viscous damping is provided. This analytical methodology allows to reduce the uncertainty of the transfer function of the platform close to the resonances of the motions and, as a consequence, it increases the accuracy of the inference approach. The second relevant contribution is the development of an alternative prior distribution, which is adopted to introduce the prior beliefs regarding the sea state in the Bayesian inference approach. It is shown that although some aspects of this novel approach require further evaluation in future work, the prior distribution developed has potential to improve the accuracy of wave estimates, and, at the same time, it significantly simplifies the calibration procedures followed by other state-of-the-art Bayesian wave inference methods.

Summing up, the inference approach proposed in this work provides the bases to use each semisubmersible oil platform, which stand as the most common type of oil platforms operated offshore Brasil, as a motion based wave sensor, thus contributing to the possible broadening of the Brazilian oceanographic measurement network.

Keywords: Semisubmersible Platform. Viscous Damping. Motion-Based Wave Inference. Bayesian Inference. Prior Distribution. Extreme Sea Conditions. 


\section{RESUMO}

A presente tese investiga a adoção de plataformas de petróleo semissubmersíveis como base para inferência das condições de onda através do monitoramento de seus movimentos. O problema em questão consiste na solução do problema inverso de comportamento em ondas; ou seja, uma vez observados os movimentos da unidade flutuante (e conhecidas suas funções de resposta de movimento), estima-se as condições de ondas que os causaram.

Este tipo de método já vem sendo empregado há anos para navios em curso e também para navios convertidos em plataformas de petróleo (os chamados FPSOs) com bons resultados. No entanto, o possível emprego de plataformas semissubmersíveis para o mesmo fim foi muito pouco explorado até o momento. Evidentemente, isso decorre da suposição de que, uma vez que essas estruturas são projetadas com o intuito primeiro de atenuar os movimentos decorrentes das ações de ondas, naturalmente elas não seriam bons sensores para esta finalidade. Os resultados apresentados nesta tese, todavia, contrariam tal suposição. De fato, as semissubmersíveis respondem de forma fraca às ondas, porém esta resposta é mensurável. Não apenas isso, mas, em comparação com os cascos de navios, esta resposta adere melhor às previsões dos modelos hidrodinâmicos lineares a partir dos quais as características da plataforma são estimadas. Ademais, o caráter eminentemente linear da resposta muitas vezes perdura inclusive para condições de ondas severas. Isto, por sua vez, torna as semissubmersíveis promissoras inclusive para a estimação de mares extremos, situação nas quais os outros tipos de sensores (boias, radares) enfrentam dificuldades. Nesta tese, a demonstração destes fatos é sustentada por um extenso conjunto de testes experimentais realizados em tanque de ondas com um modelo em escala reduzida de uma plataforma que hoje opera no Mar do Norte. Para tanto, foi empregado um método de inferência Bayesiana para estimação de ondas em navios que vem sendo desenvolvido na EPUSP há mais de dez anos. Para o estudo das semissubmersíveis o trabalho propõe duas melhorias importantes no método: A primeira consiste em um procedimento analítico para prever o amortecimento hidrodinâmico de origem viscosa dos movimentos observados do casco. Este procedimento permite reduzir as incertezas quanto à função de resposta em condições de ressonância dos movimentos com as ondas e, dessa forma, aumentar a confiabilidade do método. A segunda contribuição relevante é a proposição de uma alternativa para a chamada distribuição a priori originalmente empregada pelo método Bayesiano. Demonstra-se que, embora alguns aspectos desta nova metodologia ainda necessitem de uma avaliação adicional em trabalhos futuros, a nova distribuição tem grande potencial para melhorar a precisão das estimativas de ondas, além de simplificar de maneira significativa os procedimentos atuais de calibração do sistema de inferência.

Em suma, o método de inferência aqui proposto abre caminho para tornar cada unidade flutuante de óleo e gás do tipo semissubmersível, um dos sistemas de produção mais frequentes nas costas brasileiras, um eventual ponto de monitoramento de ondas, contribuindo então para a possível ampliação de nossas bases de medição oceanográficas.

Palavras-Chave: Plataforma Semissubmersível. Amortecimento Hidrodinâmico de Origem Viscosa. Inferência de Onda Baseada nos Movimentos Medidos. Inferência Bayesiana. Distibuição A Priori. Condições de Mares Extremas. 


\section{LIST OF FIGURES}

1.1 Directional wave spectrum. From (LLOYD, 1989) . . . . . . . . . . 2

1.2 Wave monitoring systems: Wave buoy (left) and wave radar (right). From (TECHNOLOGIES, 2018) and (OLJEMUSEUM, 2015), respectively. . . . 4

1.3 Wave buoys stations in the Gulf of Mexico. Data from (NOAA, 2018). . . 5

1.4 Wave buoys stations in the Santos, Campos and Espíritu Santo basins, located in the Southern region of the Brazilian coast. Data from (GOOS-

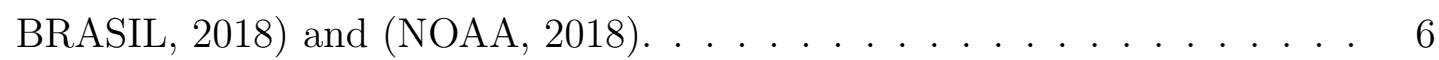

1.5 Wave buoys and O\&G floating offshore paltforms stations in the Santos, Campos and Espítu Santo basins. Data from (GOOS-BRASIL, 2018), (NOAA, 2018) and (PETROBRAS, 2018). . . . . . . . . . 8

1.6 FPSO Cidade de Ilhabela (left) and semisubmersible P-51 (right). From

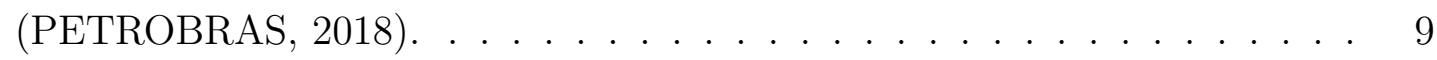

1.7 GUI of the VMB wave estimation software. From (BISPO et al., 2016). . . 11

3.1 Harmonic wave definitions. . . . . . . . . . . . . . . . . 27

3.2 Illustration of the Peak period, Mean wave period and Mean zero upcrossing period. . . . . . . . . . . . . . . . . 35

3.3 Example of cosine-squared directional spread function (left) and the power spectral density (right). . . . . . . . . . . . . . . . . 37

3.4 Example of directional energy spectrum. . . . . . . . . . . . 37

3.5 Wave spectrum models comparison. . . . . . . . . . . . . . . . . . 39

3.6 Encounter $\beta$ angle: given by the difference between the wave direction and the heading of the floating vessel. . . . . . . . . . . . . . . . 44 
3.7 Loading regimes of horizontal forces acting on the columns of the Åsgard-B semisubmersible platform in deepwater. Inspired from (CHAKRABARTI,

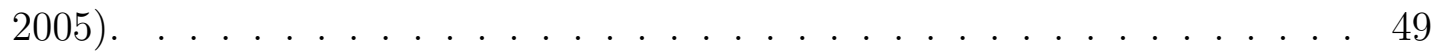

3.8 Heave motion RAO. . . . . . . . . . . . . . . . . . . . . . . . 52

3.9 Keulegan-Carpenter values estimated for the pontoons of the semisubmersible platform near resonance. . . . . . . . . . . . . . . 53

3.10 RAOs of the Åsgard-B semisubmersible platform. From left-to-right then top-to-bottom: surge, sway, heave, roll and pitch. . . . . . . . . . . . 55

3.11 RAOs of a FPSO. From left-to-right then top-to-bottom: surge, sway, heave, roll and pitch. Data from (BISPO, 2011b). . . . . . . . . . . 56

4.1 Illustration of the weighted regularization problem. . . . . . . . . . 63

4.2 Schematic representation of the algorithm adopted to estimate the matrix

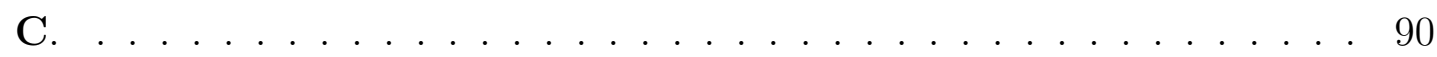

5.1 Åsgard-B floating gas production platform (left) and a sketch showing the position of the Åsgard field in the Norwegian sea (right), from (STATOIL,

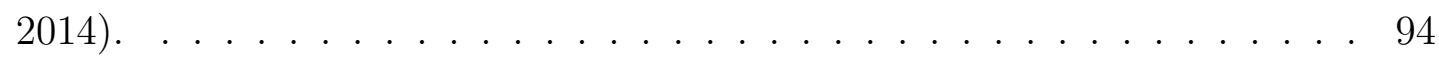

5.2 The 1:120 scaled semisubmersible platform model. . . . . . . . . . . . . 95

5.3 Higher order mesh of the semisubmersible platform. . . . . . . . . . . . . . 97

5.4 RAOs for the motions of heave, roll and pitch. . . . . . . . . . . . . . 98

5.5 Main wave direction relative to the platform. . . . . . . . . . . . . 98

5.6 RAO heave, for the heading of $\beta=180^{\circ} \ldots \ldots \ldots 10 \ldots$

5.7 Sketch of the wave basin and model (not in same scale), including the length and stiffness of the mooring lines. Adapted from Mello (2005). . . . 106

5.8 Time series of the decay tests, for heave, roll and pitch motions. . . . . . . 107

5.9 Estimated damping ratios for heave, roll, pitch and yaw (from left-to-right then top-to-bottom). . . . . . . . . . . . . . . . . 108 
5.10 Linearized heave damping factor (black points) estimated from wave tests confronted with the experimental results of decay test presented in figure 5.9 (blue triangles) which are linearly fitted with the red line.

5.11 Comparison of the (heave, roll \& pitch) RAOs estimated numerically (black line) with the experimental results, both transient (red dashed line) and regular waves with $2 \%$ of steepness (green triangles), for a heading of $135^{\circ} .110$

5.12 Time series of surface elevation of the first realization of sea condition STA-IRR-29, with $H_{s}=14.1 \mathrm{~m}$ and $T_{p}=19.3 \mathrm{~s} \ldots \ldots \ldots 113$

5.13 Picture of the model-scale of the semisubmersible paltform during one irregular wave sea condition in the TPN-USP. . . . . . . . . . . . . . 114

5.14 Recorded heave, roll and pitch motion time series for sea condition STAIRR-29.

5.15 Variability that the sea spectrum generated in the wave basin presents, for each realization, and sea conditions corresponding to tests: 2, 3, 23, 31. . . 115

5.16 Lay-out of the wave probes array (left) and picture of the array before the installation in the wave basin (right) . . . . . . . . . . . . 117

5.17 Irregular sea (full-scale) estimations using MEM for the tests: 2, 14, 25 and 32 (left-to-right then top-to-bottom) . . . . . . . . . . . . 117

5.18 Coefficient of variability of the temporal variance estimator for all the sea conditions. . . . . . . . . . . . . . . . . . . . 120

5.19 Optimal sample time for each sea condition. . . . . . . . . . . . 120

5.20 Comparison between the optimal sample time estimated for each sea conditions as proposed in Fig. 5.19 (Est. Time) and ITTC (ITTC Time). In this case the percentile levels for the results provided in Fig. 5.19 and the ones drawn using the ITTC approach are provided by the point-dashed lines $(25 \%$ and $75 \%)$ and continuous lines (50\%) in blue and black in black and blue, respectively. . . . . . . . . . . . . . . . . . . 122

5.21 Coherence function for beam sea conditions. . . . . . . . . . . . . . 125 
5.22 Coherence function for quartering seas. . . . . . . . . . . . . . . . 125

5.23 Comparison between the sea spectrum (in blue) for tests 2 and 31, top and bottom respectively, the heave coherence function (red) and the heave RAO (black). . . . . . . . . . . . . . . . . . . . 127

5.24 Results of the wave inference method estimated with different sets of RAOs for the heading $90^{\circ}$, concerning the first realization. Blue and red markers indicate whether the waves generated in the basin corresponded to a JONSWAP or Torsethaugen power spectrum, respectively. The relative errors provided in these figures are defined in Eqs. (5.9) and (5.10) . . . . . . . . 131

5.25 Results of the wave inference method estimated with different sets of RAOs for the heading $90^{\circ}$, concerning the second realization. . . . . . . . . . 132

5.26 Results of the wave inference method estimated with different sets of RAOs for the heading $135^{\circ}$, concerning the first realization. . . . . . . . . . 133

5.27 Results of the wave inference method estimated with different sets of RAOs for the heading $135^{\circ}$, concerning the second realization. . . . . . . . . . . 134

5.28 Wave spectra estimated using variable damping for the heading $90^{\circ}$, regarding the first realization of the sea conditions $2,14,25$ and $32 . \ldots 139$

5.29 Wave spectra estimated using variable damping for the heading $135^{\circ}$, regarding the first realization. . . . . . . . . . . . . . . . . . . 140

5.30 $H_{s}$ and $T_{p}$ mean relative errors from Tab. 5.6. Blue markers stand for the JONSWAP sea conditions and the red ones for those sea conditions generated by means of the Torsethaugen wave spectrum. The lines included in the plots stand as the quadratic fitting for each set of points (i.e. blue

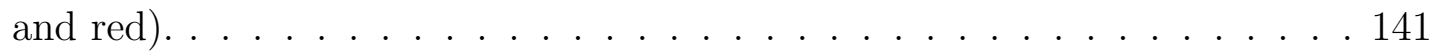

5.31 Box-plot showing the ratio between the estimated and measured mean wave period, obtained by means of the conventional approaches. . . . . . . . . . 143

5.32 Box-plot showing the ratio between the estimated and measured significant wave height, obtained by means of the conventional approach. . . . . . . . 143 
5.33 Box-plot showing the ratio between the estimated and measured mean wave direction, obtained by means of the conventional approach. . . . . . . . . . 144

5.34 Box-plot showing the ratio between the estimated and measured mean wave period, obtained by means of the alternative prior distribution. . . . . . . . 145

5.35 Box-plot showing the ratio between the estimated and measured significant wave height, obtained by means of the alternative prior distribution. . . . . 145

5.36 Box-plot showing the ratio between the estimated and measured mean wave direction, obtained by means of the alternative prior distribution. . . . . . 146

5.37 Expected energy distribution in frequency-domain for sea conditions: 6,12 , 15, 17, 30 and 31 (from left-to-right then top-to-bottom). For a heading equal to $90^{\circ} \ldots \ldots \ldots \ldots \ldots \ldots$. . . . . . . . . . . . . . . . . . . . . . . . . . . .

5.38 Expected energy distribution in frequency-domain for sea conditions: 6,12 , 15, 17, 30 and 31 (from left-to-right then top-to-bottom). For a heading equal to $135^{\circ} \ldots \ldots \ldots \ldots \ldots$. . . . . . . . . . . . . . . . . . . . . . . .

5.39 Comparison of directional wave spectra estimated with three different methods (for a heading equal to 90deg.). From left-to-right: Measured sea spectrum, the ones estimated with the conventional prior distribution and the output obtained with the alternative approach. From top-to-bottom: Sea conditions $4,12,30$ and $31 . \ldots \ldots \ldots$. . . . . . . . . . . . . . . . . .

5.40 Directional wave spectra for a platform heading equal to 135deg., estimated using three different methods. From left-to-right: Measured sea spectrum, the ones estimated with the conventional prior distribution and the output obtained with the alternative approach. From top-to-bottom: Sea conditions $4,12,30$ and $31 . \ldots \ldots \ldots \ldots$

6.1 Wave buoys and semisubmersible offshore paltforms stations in the Santos, Campos and Espíto Santo basins. Information from (GOOS-BRASIL, 2018), (NOAA, 2018) and (PETROBRAS, 2018). . . . . . . . . 158

B.1 Distribution of the surface for the first sea condition. . . . . . . . . . 177

B.2 Distribution of the surface for the once in 100 years sea condition. . . . . . 177 
B.3 Illustration of different types of spectra. Adapted from (EWANS; JONATHAN,

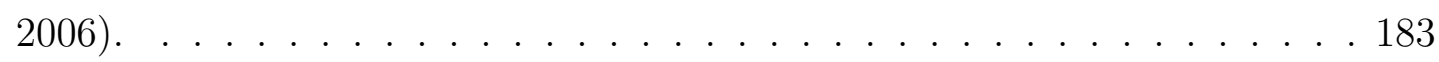

D.1 Illustration of heave RAO of motion. . . . . . . . . . . . . . 197

F.1 ANSYS-AQWA model including the submerged lines. . . . . . . . . . . . . 204

F.2 Normalized heave amplitude, for the test with wave steepness equal to $2 \%$ and including the risers and mooring lines. . . . . . . . . . . . 205

F.3 RAO heave, including the drag effects due to the submerged lines. . . . . . 206 


\section{LIST OF TABLES}

1.1 Summary of the deployment time, cost and energy uncertainty for different monitoring systems. In this table $M$ stands for million and $k$ for thousand. Data from (FILIPPELLI et al., 2015). . . . . . . . . . . 6

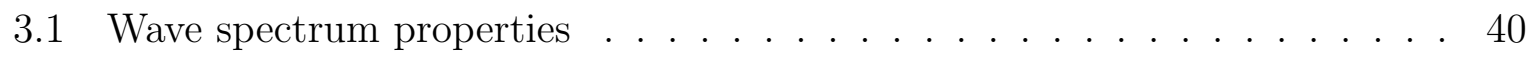

4.1 Hyperparameter values and the corresponding estimated error. . . . . . . . 64

5.1 General geometric and mass properties in full-scale and model-scale of the case study. . . . . . . . . . . . . . . . . . . . . 96

5.2 Annual probability of the input parameter used for some of the sea tests conditions. . . . . . . . . . . . . . . . . . . . 111

5.3 Input parameters used for the sea tests conditions. The wave direction in the tank was considered as $180^{\circ}$ for all the tests conditions. . . . . . . . . . 112

5.4 Comparison between the results obtained using 30min intervals over the entire span of the test (90min) and the time estimated in Fig. 5.19 for test 23 (equal to $79.1 \mathrm{~m}) . \ldots \ldots \ldots \ldots$

5.5 Mean of the relative errors measured during the experimental campaign for $H_{s}$ and $T_{1}$, regarding the ten sea conditions with the largest mean wave periods. . . . . . . . . . . . . . . . . . 137

5.6 Mean of the relative errors measured during the experimental campaign for $H_{s}$ and $T_{1}$, computed using the estimations obtained through the two realizations. . . . . . . . . . . . . . . . . . . . . 141

5.7 Mean absolute values of the mean ratios provided in Figs. 5.31 to 5.36, organized according to the mean wave period. . . . . . . . . . . . . 150

B.1 Estimated skewness for all the sea tests conditions. . . . . . . . . . . . . 179

C.1 Empirical parameter values for Torsethaugen wave spectrum. . . . . . . . . 192 


\section{NOMENCLATURE}

\section{General Rule}

Some of the symbols appear in the text representing more than one quantity. However, their actual meaning should be clear from the context.

\section{Roman Letters}

\begin{tabular}{|c|c|}
\hline A & Response Amplitude Operator matrix \\
\hline$A$ & Real part of the Response Amplitude Operator \\
\hline$A$ & Wave amplitude \\
\hline$A_{z}$ & Significant amplitude of the heave motion \\
\hline $\mathbf{A}^{+}$ & Pseudoinverse of matrix $A$ \\
\hline$a$ & Added mass \\
\hline$a_{33}$ & Heave added mass \\
\hline$B$ & Beam of the platform \\
\hline$B$ & Imaginary part of the Response Amplitude Operator \\
\hline$B$ & Measured motions of the platform \\
\hline$B$ & Potential damping \\
\hline$b$ & Linear damping coefficient \\
\hline$b_{\text {pont }}$ & Beam of the pontoon \\
\hline C & Alternative regularization weights matrix \\
\hline $\mathrm{CoV}(*)$ & Coefficient of variance \\
\hline$C_{a}$ & Added mass coefficient \\
\hline$C_{d}$ & Drag coefficient \\
\hline$c$ & Hydrostatic restoring coefficient \\
\hline$D$ & Directional spread fucntion \\
\hline$D$ & Characteristic length scale \\
\hline $\mathrm{D}$ & Regularization matrix \\
\hline$E$ & Energy density \\
\hline$E[*]$ & Expected value \\
\hline$E_{G}[*]$ & Expected value for $\mathrm{G}$ probability \\
\hline $\mathbf{e}_{j}$ & $j^{\text {th }}$ canonical basis vector \\
\hline$F$ & Force \\
\hline$F_{d}$ & Drag force \\
\hline$F_{h}$ & Radiation forces \\
\hline$F_{p}$ & Pressure forces \\
\hline$F_{h s}$ & Body weight forces \\
\hline$G$ & Generating pdf \\
\hline$G$ & Normalizing directional factor \\
\hline$g$ & Gravity constant \\
\hline $\mathbf{H}$ & Regularization weights matrix \\
\hline$H$ & Water depth \\
\hline$H_{s}$ & Significant wave height \\
\hline$h$ & Wave height \\
\hline
\end{tabular}




\begin{tabular}{|c|c|}
\hline$h(*)$ & Arbitrary probability density function \\
\hline$I$ & LK-divergence \\
\hline$J(*)$ & Objective function \\
\hline$K$ & Number of wave directions \\
\hline $\mathrm{KC}$ & Keulegan-Carpenter number \\
\hline$k$ & Thousand \\
\hline$k$ & Wave number \\
\hline$L(*)$ & Likelihood funtion \\
\hline $\mathbf{M}$ & Matrix of inertia \\
\hline$M$ & Million \\
\hline$m_{n}$ & Moment of order $n$ of $S_{\zeta \zeta}$ \\
\hline $\mathcal{N}$ & Normal distribution \\
\hline$P(*)$ & Probability of an event \\
\hline$p(*)$ & Prior probability distribution \\
\hline$p$ & Pressure \\
\hline $\operatorname{pdf}(*)$ & Probability distribution function \\
\hline$S$ & Cross sectional area of a submerged element \\
\hline$S_{j j}$ & Power spectrum of the $j^{t h}$ vessel mode response \\
\hline$S_{\eta \eta}$ & Wave density spectrum, \\
\hline$S_{\zeta \zeta}$ & Wave power spectral density function \\
\hline$T$ & Period of oscillation \\
\hline$T$ & Wave period \\
\hline$T_{p}$ & Peak period \\
\hline$T_{1}^{p}$ & Mean wave period \\
\hline$T_{2}$ & Zero up-crossing period \\
\hline$T_{\frac{1}{2}}$ & Median wave period \\
\hline$t^{2}$ & Time \\
\hline$u$ & Flow velocity \\
\hline$u_{i}$ & $i^{\text {th }}$ hyperparameter \\
\hline $\operatorname{Var}(*)$ & Variance \\
\hline$x$ & Horizontal space variable \\
\hline $\mathbf{x}$ & Unknown \\
\hline$\hat{\mathbf{x}}$ & Estimation of the unknown \\
\hline$y$ & Horizontal/Vertical space variable \\
\hline$Z$ & Complex Response Amplitude Operator \\
\hline$z$ & Vertical space variable \\
\hline
\end{tabular}

\section{Greek Letters}

$\begin{array}{cl}\beta & \text { Direction of wave incidence } \\ \gamma_{\eta \xi} & \text { Coherence function for } \zeta \text { and } \xi \\ \varepsilon & \text { Sensor noise } \\ \varepsilon_{p} & \text { Model noise } \\ \zeta & \text { Damping ratio } \\ \eta & \text { Water surface elevation } \\ \eta_{H_{s}} & \text { Relative error for } H_{s} \\ \eta_{T_{1}} & \text { Relative error for } T_{1}\end{array}$




$\begin{array}{cl}\eta_{\theta} & \text { Relative error for } \theta \\ \theta & \text { Mean wave direciton } \\ \theta & \text { Rotation around y-axis } \\ \lambda & \text { Hyperparameter } \\ \lambda & \text { Wavelength } \\ \xi & \text { Amplitudes of the vessel motions } \\ \rho & \text { Water density contant } \\ \sigma & \text { Variance } \\ \sigma_{\varepsilon} & \text { Variance of the sensor noise } \\ \sigma_{B} & \text { Variance of the measured motion } \\ \sigma_{p} & \text { Variance of the prior distribution } \\ \sigma_{x} & \text { Variance of the unknown } \\ \phi & \text { Random phase shift } \\ \phi & \text { Rotation around x-axis } \\ \phi & \text { Velocity potential } \\ \varphi & \text { Phase angle of the Response Amplitude Operator } \\ \psi & \text { Rotation around z-axis } \\ \omega & \text { Wave Frequency } \\ \omega_{n} & \text { Natural frequency }\end{array}$

\section{List of Abbreviations and Acronyms}

$\begin{array}{ll}\text { ABIC } & \text { Akaike's Bayesian Information Criterion } \\ \text { ANP } & \text { National Petroleum Agency } \\ \text { BC } & \text { Boundary Conditions } \\ \text { BS } & \text { Bretschneider wave spectrum } \\ \text { dof } & \text { Degree of freedom } \\ \text { EPUSP } & \text { Polytechnic School of university of São Paulo } \\ \text { FFT } & \text { Fast Fourier Transform } \\ \text { FPSO } & \text { Floating Production Storage and Offloading } \\ \text { GEV } & \text { General Extreme Values } \\ \text { GM } & \text { Metacentric height } \\ \text { GUI } & \text { Graphical User Interface } \\ \text { ITTC } & \text { International Towing Tank Conference } \\ \text { KC } & \text { Keulegan-Carpenter } \\ \text { MEM } & \text { Maximum Entropy Method } \\ \text { MLE } & \text { Maximum Likelihood Estimation } \\ \text { Metocean } & \text { Meteorologic and physical oceanographic } \\ \text { O\&G } & \text { Oil and Gas } \\ \text { PDS } & \text { Meteorologic and physical oceanographic } \\ \text { PM } & \text { Pierson-Moskowitz wave spectrum } \\ \text { RAO } & \text { Response Amplitude Operator } \\ \text { SWL } & \text { Still Water Line } \\ \text { TPN-USP } & \text { Numerical Offshore Tank } \\ \text { USP } & \text { Universidade de São Paulo } \\ \text { VMB } & \text { Vessel Motion-Based }\end{array}$




\section{CONTENTS}

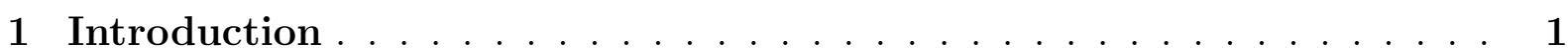

1.1 Motivation . . . . . . . . . . . . . . . . . . . 1

1.1.1 Wave estimation in the sea: The state-of-the-art . . . . . . . . . 2

1.1.2 Relevance of the VMB wave inference method for the industry . . . 7

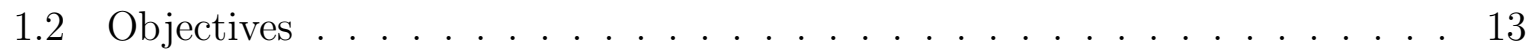

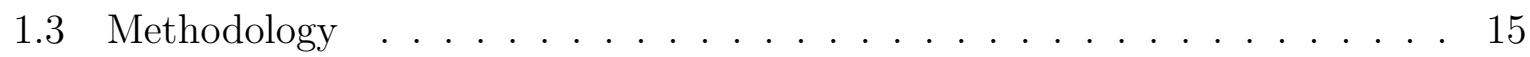

1.3.1 The non-parametric approach: The Bayesian VMB wave inference . 16

1.3.2 The role of the prior distribution in the Bayesian VMB wave inference 16

1.3.3 Modelling non-linearities of the resonant motions . . . . . . . . . . 17

1.3.4 Assessing the accuracy of the VMB wave inference methodology based on a semisubmersible platform . . . . . . . . . . . . . 18

1.4 Text structure . . . . . . . . . . . . . . . . . . . . . . . . . 19

2 Literature Review . . . . . . . . . . . . . . . . . . . . . 20

3 Linear Modelling of Ocean Waves and Wave Induced Responses of Floating Offshore Structures . . . . . . . . . . . . . 26

3.1 Introduction . . . . . . . . . . . . . . . 26

3.2 Description of ocean waves . . . . . . . . . . . . . . . . 26

3.2.1 Regular waves . . . . . . . . . . . . . . . . . . . . . 27

3.2 .2 Irregular waves . . . . . . . . . . . . . . . . 30

3.2.2.1 Note on standard wave spectrum models . . . . . . . . . . 35

3.3 Ship motions in waves . . . . . . . . . . . . . . . . . 41 
3.3.1 Viscous damping effects . . . . . . . . . . . . . . . . . . 45

3.3.2 Note on the motions of semisubmersible platforms in waves . . . . . 47

3.4 The semisubmersible platform as a VMB wave sensor . . . . . . . . . . . 54

4 Bayesian Inference . . . . . . . . . . . . . . . . . . . . . . . . 59

4.1 The Bayesian paradigm . . . . . . . . . . . . . . . . . 59

4.2 Bayesian modelling procedure and ill-conditioned problems . . . . . . . . . 60

4.3 Data distributions . . . . . . . . . . . . . . . . . 66

4.4 Prior distributions . . . . . . . . . . . . . . . . . . . . . 74

4.4 .1 Conventional approach . . . . . . . . . . . . . . . . 75

4.4.2 Alternative prior distribution ............... 81

5 Case Study: The Åsgard-B Semisubmersible Platform . . . . . . . . . . 92

5.1 General description . . . . . . . . . . . . . . . . . . . . 92

5.2 Numerical modelling: Motion RAOs and external damping . . . . . . . . . 96

5.2 .1 Introduction . . . . . . . . . . . . . . . . 96

5.2 .2 Frequency-domain analysis . . . . . . . . . . . . . . . 97

5.2 .3 Time-domain analysis . . . . . . . . . . . . . . . . . 99

5.2.3.1 Drag effects arising from the pontoons . . . . . . . . 99

5.2.4 Viscous drag forces and damping linearization . . . . . . . . . . . 102

5.3 Experimental setup and tests description . . . . . . . . . . . . . 104

5.3 .1 General . . . . . . . . . . . . . . . . . . 104

5.3.2 Motion decay tests . . . . . . . . . . . . . . . . . 105

5.3 .3 Wave tests . . . . . . . . . . . . . . . . 108

5.3.4 Wave spectra estimation from wave probes . . . . . . . . . 115

5.4 Directional wave spectrum estimation based on model test data . . . . . . 118 
5.4.1 Selection of the optimal sample time for each sea condition . . . . . 118

5.4.2 An analysis of the adequacy of the heave-roll-pitch motion basis for VMB wave inference based on coherence functions . . . . . . . . . . 124

5.4.3 Wave inference using the conventional prior distribution . . . . . 128

5.4.4 Wave Inference using the alternative prior distribution . . . . . . . 142

6 Conclusions and Future Work . . . . . . . . . . . . . . 154

6.1 Major findings . . . . . . . . . . . . . . . . . . 159

6.2 Future work . . . . . . . . . . . . . . . . . . 160

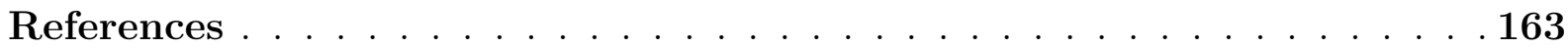

Appendix A - Sinusoidal Wave as a Random Process . . . . . . . . . 171

Appendix B - Statistical Description of Waves . . . . . . . . . . . 175

B.1 Gaussianity . . . . . . . . . . . . . . . . . 175

B.2 Ergodicity . . . . . . . . . . . . . . . . . 178

B.3 Stationarity . . . . . . . . . . . . . . . . 180

B.4 Optimal sample time . . . . . . . . . . . . . . 180

Appendix C - Standard Models for Wave Spectrum . . . . . . . 188

Appendix D - Analysis of the Heave Motion . . . . . . . . . . . . 193

Appendix E - Construction of a Smooth Prior Distribution . . . . . . . 199

Appendix F - Drag Effects arising from Mooring Lines and Risers . . . . 203 


\section{INTRODUCTION}

\subsection{Motivation}

The knowledge of the sea conditions is of central importance for a wide range of offshore and nearshore operations, engineering design and validation of forecasts of extreme wave events. In general, field measurements of the sea conditions are collected by research institutions and governmental organizations. Notwithstanding this, a significant amount of these meteorological and physical oceanographic data (also known as metocean data) is collected by private industry, in particular by Oil and Gas (O\&G) companies.

As a matter of fact, observed metocean data and analyses provide the O\&G industry with essential information and knowledge for the design and engineering of offshore installations, such as platforms and pipelines, and decision support systems. For the sake of illustration of the main benefits related to the use of ocean data, some examples extracted from (SHAW et al., 1999) are provided:

- A $5 \%$ reduction in design wave height translates into a reduction in steel costs that may range between $5 \%$ and $10 \%$;

- Decision support systems (which are crucial for a proper operational management) adopted during the installation and operation of offshore platforms are based on weather forecasts and real-time metocean data;

- The selection of the most convenient platform type for field exploration/production is usually based on historical sea data measurements.

The assessments and discussions regarding the research carried out and presented in this work are expected to represent a small contribution towards the development of the 
a more sophisticated and efficient network of wave monitoring systems, through the use of a semisubmersible platform as a motion based sea wave sensor.

\subsubsection{Wave estimation in the sea: The state-of-the-art}

For the purpose of this thesis, it is important to bear in mind the general principles and theory of wave measuring systems. For readers not familiar with such devices, a general overview of the main technologies is provided, including the wave buoys and wave radars, but this overview is by no means exhaustive. (BARSTOW et al., 2005) stands as a reference for the readers interested in a comprehensive description of the wave measuring technologies assessed below.

Wave conditions in the ocean can be characterized according to the data collected through wave measurement devices. Nevertheless, one must remember that the sea surface elevation cannot be described by means of an explicit mathematical model and, as a consequence, it is assumed to be a time-space random process (DENIS; PIERSON, 1953). Hence, to properly describe the sea state, the directional energy wave density spectrum is commonly adopted. The directional energy wave density spectrum, also know as directional wave spectrum, provides information regarding the distribution of the wave energy in both frequency and direction domain and it is estimated using the data collected through sea wave monitoring systems. Figure 1.1 shows a simple illustration of the main features that a directional wave spectrum presents.

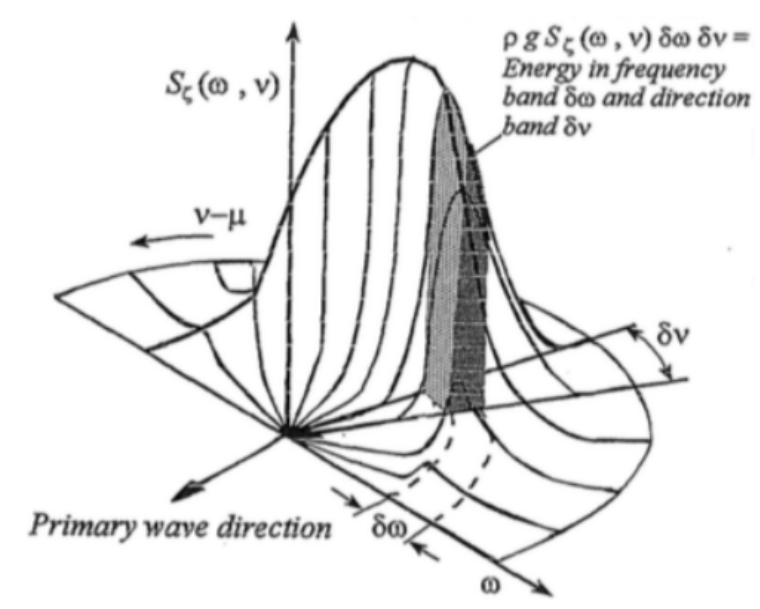

Figure 1.1: Directional wave spectrum. From (LLOYD, 1989). 
In general, on-site measurements of ocean data are obtained through moored buoys, which enclose a wide range of models. In (BARSTOW et al., 2005) the authors followed a categorization based on the variables recorded by these devices (e.g. acceleration, vertical displacement, etc.) resulting in the following types of wave buoys: (i) heave-roll-pitch buoys, (ii) displacement buoys and (iii) GPS buoys. The earliest reference to using a wave buoy for directional wave measurements appeared in (BARBER; URSELL, 1948), but it was not until some years later that Longuet-Higgins, Cartwright and Smith (1963) provided a systematical discussion and characterization of the main fundamentals of these systems, which rely on the use of the measured motions of the buoy as a basis to estimate the wave spectrum and the associated sea state parameters. The relevance of the wave buoys as a wave measurement systems is attested by several works, such as the WADIC project (ALLENDER et al., 1989), that presented the results of a one-year field trial in the North Sea and included a comprehensive analysis of the data obtained with five different wave buoys measurement systems.

During the past decades these devices have been complemented by new technologies. An example of these technologies is the wave radar measuring system, whose general principle is well known. These wave radars are generally classified based on their working frequency band. Thus, radars with HF and VHF bands are usually under $300 \mathrm{MHz}$. Regarding the navigation radars, the preferred band corresponds to the X-band ( 8 to 12 GHz.), which requires very small antennas due to the fact that the size of antennas is related to the length of the signal (higher frequencies require smaller antennas). Several works have been developed using radar data to characterize the wave conditions, such as (MAGNUSSON, 2008), where the author used wave radar data to forecast extreme wave condition in central North Sea. (FUCILE et al., 2016) is another interesting work regarding the wave inference using radar data, in this case the authors provided two analysis techniques based on measurements collected using radar imaging. An illustration of wave buoy and wave radar measurement systems is provided in Fig. 1.2.

At the same time, there are several difficulties associated with the measuring of the wave conditions through these wave monitoring systems. Indeed, a drawback arises in extreme weather conditions when these systems may render inaccurate measurements. For instance, wave buoys may be dragged through or swerve around the 3D peaks of waves (ALLENDER et al., 1989). Furthermore, the accuracy of the buoy displace- 

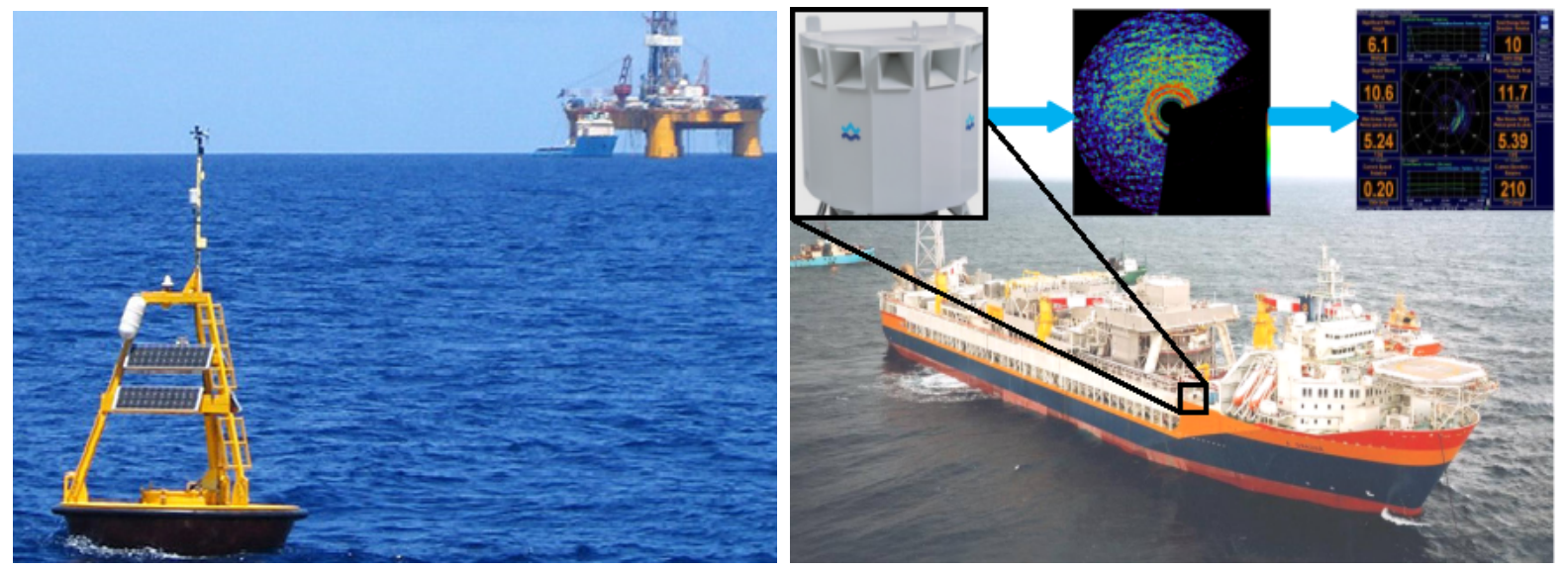

Figure 1.2: Wave monitoring systems: Wave buoy (left) and wave radar (right). From (TECHNOLOGIES, 2018) and (OLJEMUSEUM, 2015), respectively.

ments decreases when long swell wave energy (> 20s) is present (ESSEN; EWANS; MCCONOCHIE, 2018). In addition, eventual presence of a spike in the raw accelerometer data may produce erratic maximum wave height measurements (MACKAY, 2009). Rough sea states and adverse weather conditions are also among the possible sources of errors in wave measurements from wave radar systems, since errors can be attributed to the shadowing effects produced by rain, large waves or sea spray, as well as the inherent bias of the system that can happen to increase under extreme sea conditions (MAGNUSSON, 2008).

Comparisons between estimations obtained with wave buoys and wave radars have shown some important discrepancies,see for example (DURRANT; GREENSLADE; SIMMONDS, 2009; CHEN et al., 2013) and (WIJAYA, 2009), highlighting the inherent biasses and differences in the performance of these sensors in different sea conditions. Therefore, aiming at estimate the directional wave spectrum, several assumptions must be made, which should be different from one instrument to another. This aspect of the wave monitoring systems is well illustrated in (BARSTOW et al., 2005), where the authors pointed out the importance of understanding the main strengths and weaknesses of each measurement system in order to judge which parameters of the wave spectrum can be accurately estimated.

Returning to the wave buoys, nowadays they are in widespread use as they are the most common monitoring technology. This fact is well depicted by the number of these devices deployed within the major O\&G fields. For instance, Fig. 1.3 shows the moored 
wave buoys currently operating in the Gulf of Mexico. This figure includes more than 150 of these devices which are operated by governmental and private institutions. These wave buoys are concentrated in an area equivalent to 1 million square kilometers (the upper half part of the Gulf of Mexico) with a water depth ranging from $300 \mathrm{~m}$ to $2150 \mathrm{~m}$, where the main $\mathrm{O} \& \mathrm{G}$ exploration and production operations are carried out.

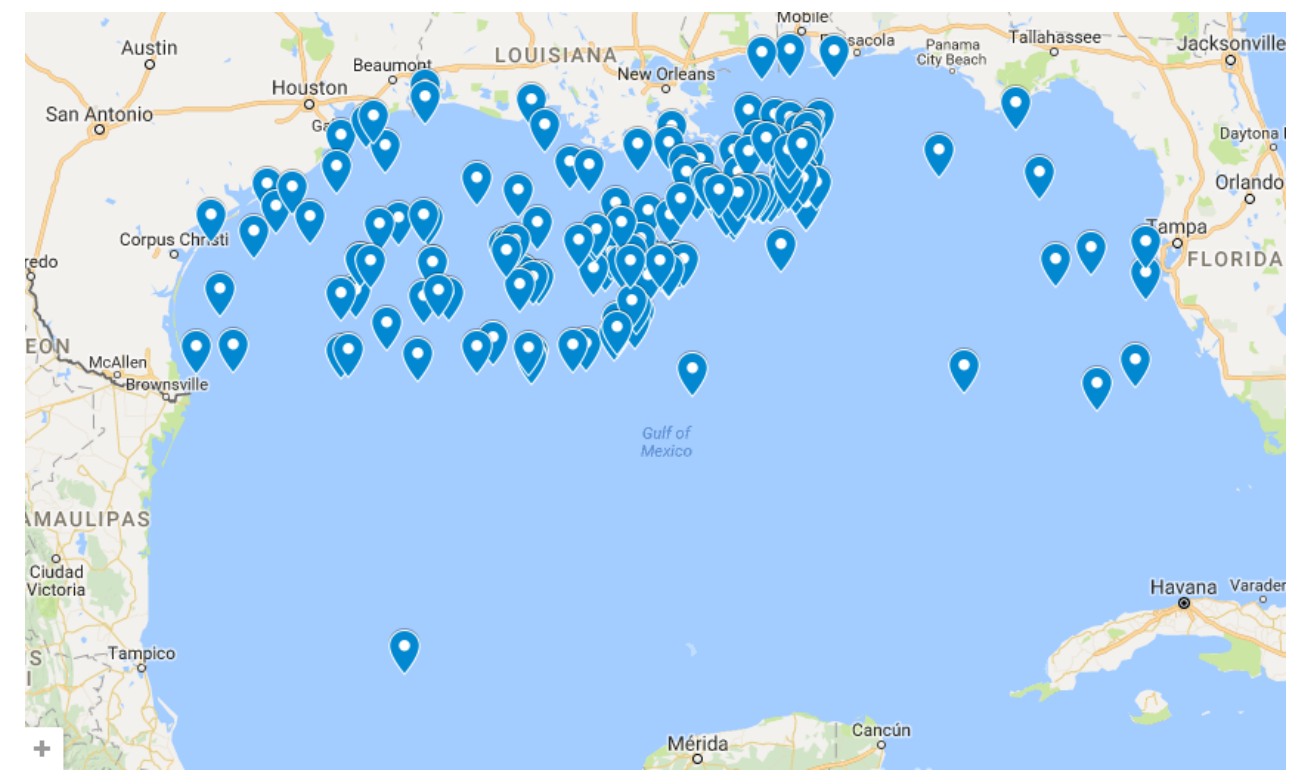

Figure 1.3: Wave buoys stations in the Gulf of Mexico. Data from (NOAA, 2018).

There are several reasons that have had a significant impact on the adoption of these devices. Table 1.1, extracted from (FILIPPELLI et al., 2015), shows the most relevant factors referring to the cost range, estimate deployment time and net energy uncertainty for different wave measuring systems. The values provided in this table include the estimated cost of the systems as well as the installation and operation for a time span of 3 years. Nonetheless, these systems usually require a calibration campaign, that generates an extra-cost that varies between $5 \%$ and $10 \%$ of the total cost of the system (FILIPPELLI et al., 2015). From these data, one may realize that the measurement systems most commonly adopted (wave buoys) present the lower prices and deployment times compared to other systems, although in some cases these advantages may be obtained at the cost of increasing the energy uncertainty.

Even though the advantages of using the wave monitoring systems seem clear (technical decisions are made, based on the collected metocean data, on whether or not spend many millions of dollars while the cost involved in collecting these data are several orders 
Table 1.1: Summary of the deployment time, cost and energy uncertainty for different monitoring systems. In this table $M$ stands for million and $k$ for thousand. Data from (FILIPPELLI et al., 2015).

\begin{tabular}{cccc}
\hline Monitoring System & Deployment time & Cost Range & Energy Uncertainty \\
\hline Hub-Height Tower/Platform & 24 months & $\$ 4.5-13 \mathrm{M}$ & $5.6 \%$ to $7.3 \%$ \\
Buoy-Based Radar & 12 months & $\$ 0.8-2.8 \mathrm{M}$ & $6.4 \%$ to $8.1 \%$ \\
Platform-Based Radar & 6 months & $\$ 300-550 k$ & $5.6 \%$ to $7.3 \%$ \\
Weather Buoy & 4 months & $\$ 150-300 k$ & $2 \%$ to $11.8 \%$ \\
\hline
\end{tabular}

of magnitude lower), some regions in the world containing major oil fields are not yet characterized by a proper network of wave monitoring sensors. As an example, Fig. 1.4 shows the moored buoys installed offshore the Brazilian Southern region. This region includes the Santos, Campos and Espírito Santo basins, which represent the major part of the Brazilian O\&G reserves and account for more than 60 bilion barrels of oil.

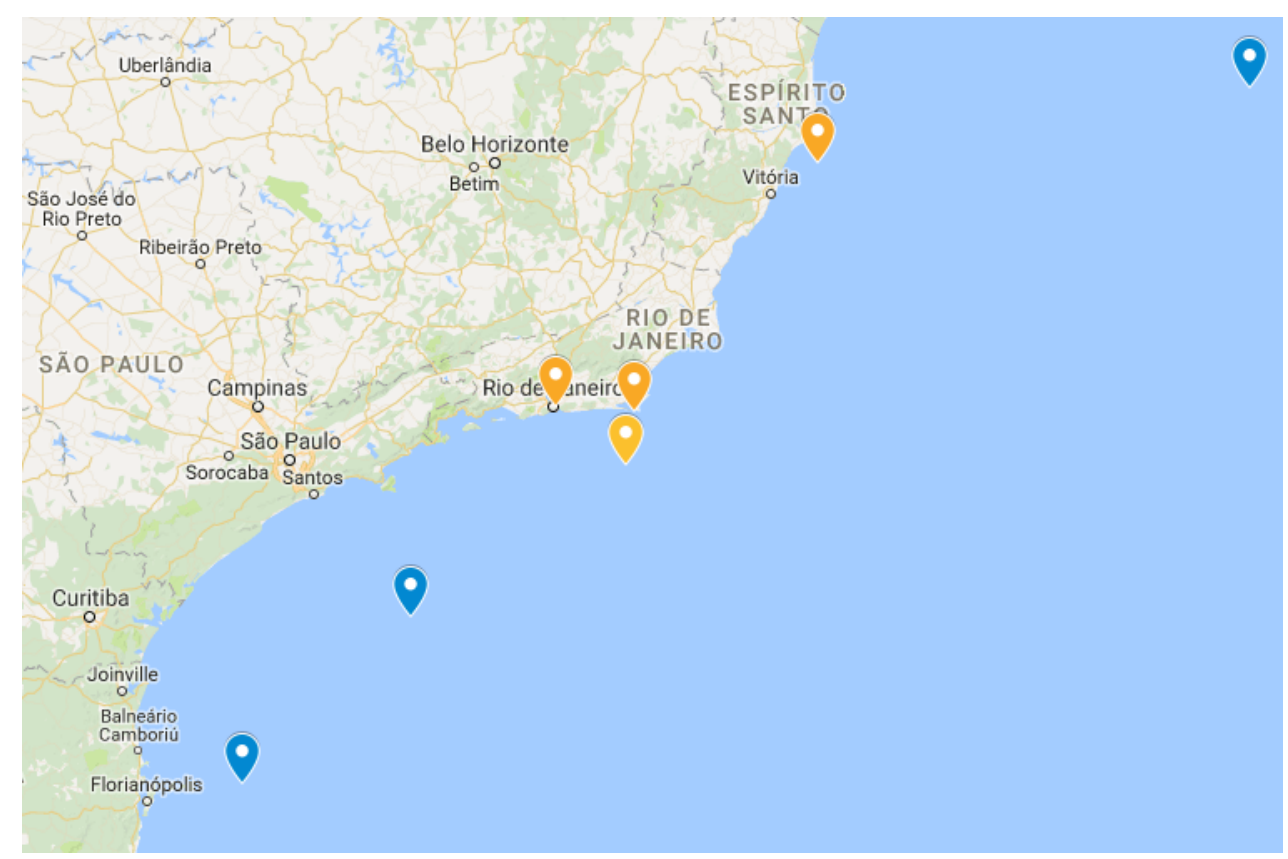

Figure 1.4: Wave buoys stations in the Santos, Campos and Espíritu Santo basins, located in the Southern region of the Brazilian coast. Data from (GOOS-BRASIL, 2018) and (NOAA, 2018).

The offshore area included in Fig. 1.4 is equivalent to 1,2 millions of square kilometers, where the maximum water depth is about $3000 \mathrm{~m}$. This figure provides the moored buoys operated by governmental and private organizations. Differently from the Gulf of Mexico, in this area only seven buoys are currently installed and four of them are under mainte- 
nance operations (orange markers) at the current date (25/08/2018). Thus, only 3 buoys are monitoring an area that is equivalent in extension and relevance to the one assessed previously in the Gulf of Mexico (where more than 150 of these devices are operating). The lack of a proper monitoring network of wave buoys can be explained by several reasons, such as the technical challenges that arise during the installation/manteinance of these devices in deep and ultra-deep water conditions ${ }^{1}$ and the fact that $O \& G$ industries began the development of their deep water activities in part of these fields during the late 90s and early 2000s.

An alternative to overcome the lack of wave measurement systems would be the use of the O\&G floating offshore platforms operating in the Brazilian coast as wave sensors. The main idea here is to complement the wave measurements obtained through the moored buoys with the sea state estimations computed based on the measured motions of the offshore platforms. Thus, for example, if all the floating platforms currently operated by Petrobras in the region assessed previously in Fig. 1.4 were adopted as wave sensors, the resulting network of monitoring systems would be composed by more than 40 sensors. This is illustrated in Fig. 1.4, were the network of waves buoys shown in Fig. 1.4 is complemented by the location of the floating O\&G offshore platforms operated by Petrobras.

The adoption of moored floating offshore platforms as on-site wave sensors to improve the wave measurement network in the Brazilian coast was an idea initially proposed in 1999, by the Naval Architecture and Ocean Engineering Department along with the Mechatronics Engineering Department of the Polytechnic School of University of São Paulo (EPUSP). The relevance and the main characteristics of this approach, also known as vessel motion-based wave inference (or for short, VMB wave inference) are properly addressed in the following section.

\subsubsection{Relevance of the VMB wave inference method for the in- dustry}

In the 1980s the firsts works assessing the VMB wave inference appeared, e.g. (WEBSTER; DILLINGHAM, 1981), mainly using short records of data for the analysis. Other works regarding this research topic were developed during the late 90s and early 2000s,

\footnotetext{
${ }^{1}$ Deep water is considered to be anything more than 1500 meters and the top end of ultra deep water comes at around 2133 meters.
} 


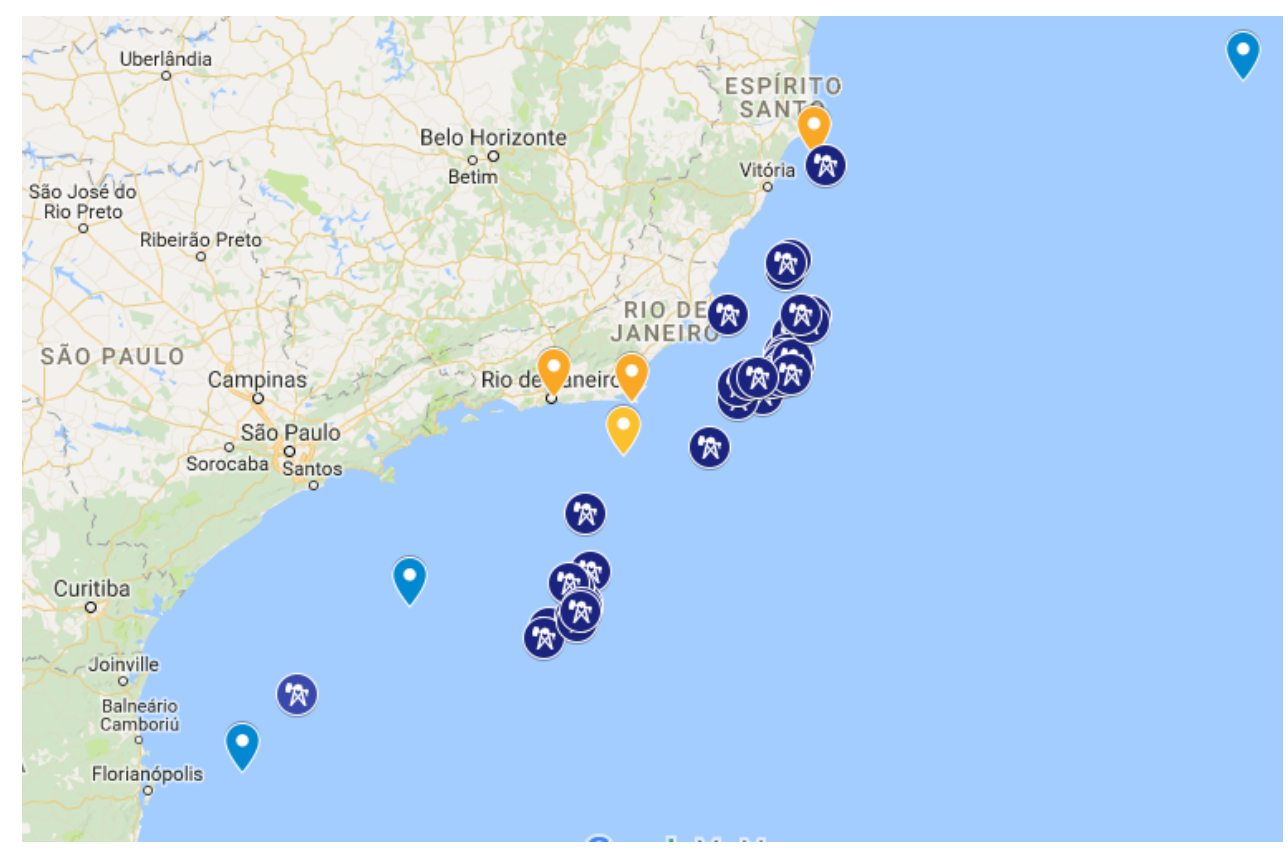

Figure 1.5: Wave buoys and O\&G floating offshore paltforms stations in the Santos, Campos and Espítu Santo basins. Data from (GOOS-BRASIL, 2018), (NOAA, 2018) and (PETROBRAS, 2018).

when the computing power available made the adoption of this method practical. These improvements lead to the first reliable application of the VMB wave inference, carried out using a container ship with forward speed. A detailed and comprehensive description of the application of this technique for wave inference in the shipping industry is provided in (NIELSEN, 2005).

Simultaneously, as it has been pointed out in the previous section, at EPUSP an extensive research program was being done to assess the use of the floating offshore platform for on-site VMB wave inference. Due to the central importance of this long-term research topic, the entire research program was supported by Petrobras in the years followed. In order to carry out this research, Floating Production Storage and Offloading (FPSO) and semisubmersible type platforms were evaluated as bases for VMB wave inference before the starting of the research campaign (Fig. 1.6 provides an illustration of both types of offshore structures). Finally, the former was selected over the later as a consequence of the following reasons:

- The hydrodynamical properties of the semisubmersible platforms as well as their massive dimensions result in motions with small or non-significant amplitudes. 
Hence, it was assumed that this type of platforms will not have the appropriate behavior for being used as wave sensor. Nevertheless, this assumption (which was accepted at the moment) was in fact inaccurate as it is shown in this thesis;

- Preceding works on VMB wave inference had been carried out in ship-shaped vessels, obtaining promising results for a several range of sea conditions;

- At that time, FPSO offshore platforms were already a common type of offshore platform operated by Petrobras in the Brazilian offshore waters, specially in the Campos basin.

As a result of this long term research, several works have been published, e.g. (TANNURI et al., 2003; SIMOS et al., 2010, 2012) and (BISPO et al., 2012), where the authors carried out an assessment of the use of a moored FPSO as a VMB wave sensor. The results provided in these works (obtained during model-scale and full-scale experimental campaigns) show that the FPSO type platforms can be used as reliable wave sensors.
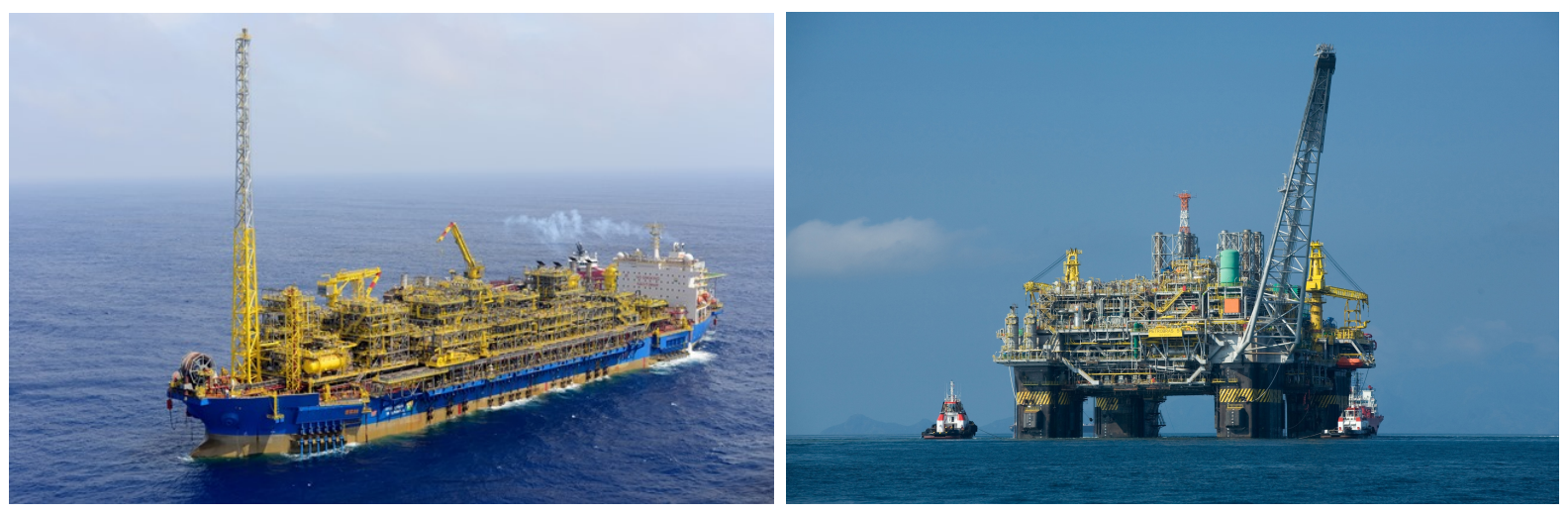

Figure 1.6: FPSO Cidade de Ilhabela (left) and semisubmersible P-51 (right). From (PETROBRAS, 2018).

A common feature of all these VMB wave inference works is the adoption of the Bayesian inference method to solve the inverse problem, which is by nature ill-conditioned. The adoption of this statistical approach allows to take into account measurements and model uncertainties in the inverse problem, avoiding misleading estimations of the unknown (BERNARDO; SMITH, 2006). Hence, the outcome of the problem (the posterior distribution of the quantity of interest, in this case the wave spectrum) combines the information provided by the measured motions of the vessel with relevant available prior information about the sea conditions. In general, the prior information for VMB wave 
inference is limited to: (i) assume that the difference between the spectra of the measured motions and the estimated ones is Gaussian distributed and that (ii) the sea spectrum inferred must be characterized by a certain level of smoothness (ISEKI; OHTSU, 2000). It is important to highlight that the pertinence of the prior information and its relevance on the output of the problem is tuned through a set of prior parameters also known as hyperparameters.

As for the other wave measurement systems, the VMB wave inference is also characterized by some limitations. The major shortcoming of the method is that the vessel acts as a kind of low-pass filter, filtering the wave components that do not excite the vessels responses (mainly high frequency ones). Therefore, the larger the vessel, or the lower its response in waves, the more restricted will be the estimation range. As an illustration of this limitation, when the estimation is based on a very large vessel, such as a FPSO platform with a VLCC hull, previous studies (BISPO et al., 2016) have shown that wave conditions may be well captured by the method only for peak periods above 8 seconds. For this reason, the VMB wave inference approach is not intended for providing broad oceanographic records, but rather to be used as a means for identifying wave conditions that impose considerable motions on the vessel on which the system is based on. A possible overcome to this drawback is assessed in (SOUZA et al., 2018), where the authors combined the Bayesian VMB wave inference and the use of on-board wave probes; following the technique discussed in (PINKSTER et al., 1978) to measure the high-order components of the wave spectrum by means of wave gauges installed around the hull. This combination of different approaches resulted in a novel methodology that aims at extending the range of wave frequencies that can be estimated through VMB wave inference, including the high-frequency ones.

In the year 2014, the VMB wave inference research program carried out at University of São Paulo (USP) consolidated an important milestone with the starting of an extensive research project, supported by EQUINOR (ex-STATOIL) in partnership with Petrobras and the Brazilian National Petroleum Agency (ANP). One of the topics envisioned in this partnership was the assessment of the use of a large semisubmersible platform as a VMB sea wave sensor. This idea proposed by the EQUINOR's department of physical oceanography had as a main objective the inference of extreme sea conditions, that would induce significant motions on a semisubmersible platform increasing its reliability as VMB 
inference sensor. This research project also enclosed the assessment of the use of waveprobes to increase the reliability of the VMB wave inference method in the high frequencydomain and a two years real scale monitoring campaign in the Peregrino field, located in the southwest part of the Campos Basin, using the measured motions onboard of the Peregrino FPSO, which is operated by EQUINOR and its partner Sinochem. Another improvement related to the project was the development of a fully working graphical user interface (GUI), that allows an effective operation and control of the VMB wave inference algorithm. Figure 1.7 provides an example of the developed GUI.

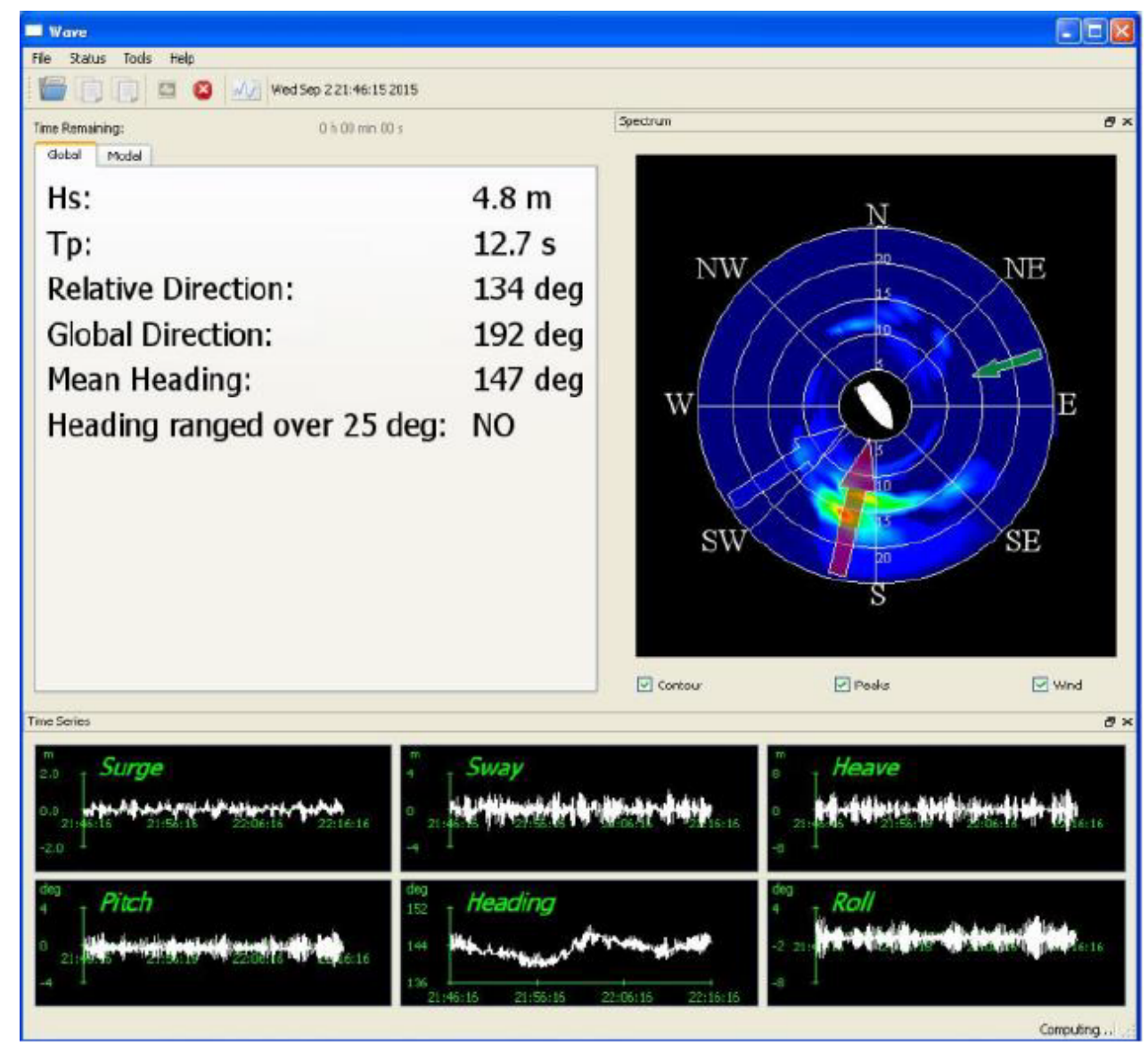

Figure 1.7: GUI of the VMB wave estimation software. From (BISPO et al., 2016).

Regarding the idea proposed by EQUINOR of using a semisubmersible platform as a VMB wave inference sensor, it was assessed by means of a dedicated model-scale experimental campaign carried out in the wave basin of the USP's numerical offshore tank (TPN-USP) using a scaled model of the Åsgard-B semisubmersible platform, currently operating offshore Norway. The results of the tests carried out at the TPN-USP are presented in this thesis, showing the reliability of this type of platforms for VMB wave 
inference not only for severe sea conditions but also for mild sea states.

For the reader interested in the results obtained through this project, some publications already available are (BISPO et al., 2016; MAS-SOLER et al., 2017; SOUZA et al., 2018) and (MAS-SOLER; SIMOS; TANNURI, 2018).

Summing up, it can be stated that the growing interest in the VMB wave inference approach comes from the accuracy of the estimations obtained, the technical advantages of the method as well as the simplicity of the hardware required on-board (mainly composed by an ordinary set of accelerometers connected to a consumer-level PC, hardware that the major part of the O\&G offshore platforms already have installed on-board). These practical aspects guarantee a lower initial investment, compared to other technologies, such as radar systems or wave buoys, and uncomplicated maintenance of the system. From the point of view of the offshore O\&G industry, the use of the VMB wave inference method has several advantages:

- Ships and offshore platforms can work together to provide worldwide information about the sea conditions, even from remote areas, at anytime. This feature could improve the exploration activities in more remote offshore areas as well as in deep or ultra-deep water oil fields, where other measuring devices may presents some limitations regarding their installation and operation. The latter application may have significant importance for the exploration and production activities carried out offshore the Brazilian coast, where the major oil and gas reservoirs are located in deep or ultra-deep water conditions;

- VMB wave inference may provide real-time information about the sea condition, that is crucial for a proper operational management of an offshore platform. In this case, the use of the VMB wave inference may result in significant improvements in many offshore activities, such as drilling operations, shuttle tanker operations, installation of the topsides facilities relative to floating or fixed platforms and in the assessment of the downtime for production facilities.

As a result, the VMB wave inference has become the object of investigation both in shipping and the O\&G sectors, see for example (MAS-SOLER; SIMOS; TANNURI, 2018; NIELSEN; BRODTKORB; SØRENSEN, 2018) and (NIELSEN, 2018), as a reference 
of the last developments attained in this research topic. As a matter of fact, due to the remarkable results obtained using the inference algorithm developed at EPUSP, Petrobras has started a new project aiming to test and implement the VMB wave inference software during its offshore operations.

Finally, it is important to highlight that the main motivations for this thesis not only come from the general practical advantages of the VMB wave inference but also from the fact that although a semisubmersible is designed for responding only weakly to the incoming waves, it will still present significant linear motions in more severe wave conditions and, in these situations, it may perhaps provide an interesting complement to the readings of other devices. This would guarantee the reliability and accuracy of the method even in harsh environmental conditions, that are of important relevance for offshore projects. Nevertheless, one of the challenges arising from the use of the VMB wave inference approach to estimate extreme sea conditions is the possible lack of linearity between the induced motions of the platform and the wave exciting forces. For this reason, non-linear effects and their implications should be carefully analyzed in order to improve the robustness of the estimations and avoid misleading inferred sea statistics.

\subsection{Objectives}

The use of a large semisubmersible platform as a VMB wave sensor is investigated in the present thesis by means of a dedicated model-scale experimental campaign. In order to fully understand the major characteristics of the dynamics of a semisubmersible platform in various sea conditions, ranging from mild to extremely rough sea states, the model of the system has been set as simple as possible, only including wave exciting forces. Accordingly, risers or mooring lines have not been modelled during the experimental campaign. The objectives herein are set aiming at addressing the main motivation referred to in the previous section.

1. Asses if the Bayesian VMB wave inference method can be used in a robust way to accurately infere severe wave conditions based on the measured motions of a large semisubmersible platform.

For this purpose the Bayesian inference approach proposed by Tannuri et al. (2003) 
has been adopted. Answers to the following questions are sought:

(a) Are the assumptions, regarding the linear excitation-responses (NIELSEN, 2005) along with the properties of the wave spectrum (ISEKI; OHTSU, 2000), valid for the use of a semisubmersible platform as a wave sensor?

(b) Is the procedure adopted by Tannuri et al. (2003) to determine the prior distribution sufficiently robust to properly control the uncertainties of the model induced mainly by non-linear effects?

(c) What are the most relevant sea conditions to be tested in the wave basin in order to provide a reliable evaluation of the use of the semisubmersible platform as a VMB wave sensor?

(d) Are the sea conditions tested during the experimental campaign accurately estimated through the method?

2. Discuss the selection of the prior distribution and propose an alternative approach aiming at increasing the accuracy of the estimations.

Regarding this objective, a brief discussion of the different prior distributions has been conducted, including the approaches adopted in (ISEKI; OHTSU, 2000; NIELSEN, 2008) and (TANNURI et al., 2003). Also, a new prior distribution alternative to the previous ones is proposed. Through this, answers to the following questions will be sought:

(a) What are the main characteristics of the various prior distributions in use nowadays for wave inference?

(b) Which is the most appropriate prior distribution, among the ones compared, for the current problem?

(c) How can an alternative prior distribution provide practical improvements for the method?

3. Introduce an appropriate procedure to model the response amplitude operators and deal with the typical non-linearities of the resonant motions.

Previously, it has been highlighted that one of the challenges arising from using a semisubmersible platform as a VMB wave sensor to estimate severe sea conditions is 
that non-linear responses should be properly accounted for. In this regard, answers to the following questions will be sought:

(a) Which motions are expected to be more sensitive to non-linear effects and how do they impact the wave estimations?

(b) What are the sources of such non-linearities?

(c) Can the estimations of the VMB wave inference be improved by means of a simple damping model that account for these non-linearities?

4. Evaluate the limits, in terms of the accuracy of the estimations of the motion-based wave inference method using the measured motions of a model-scale semisubmersible platform.

During the experimental campaign only long crested waves have been simulated in the wave basin. Since these tests do not include current and wind effects, the model of the platform has been kept as simple as possible, without including submerged lines (such as risers and mooring lines) nor the topside. However, in extreme sea conditions, other phenomena, such as wind and current effects, may excite the platform motions. Consequently, the results obtained during the experimental campaign may be not enough representative for extreme weather conditions. Accordingly, the answers to the following questions will be sought:

(a) What are the potential effects of other environmental conditions, such as current or wind, on the responses of the semisubmersible platform?

(b) Do the submerged lines have a substantial influence on the motions of the semisubmersible platform within the frequency range of interest?

(c) Are there other limitations of the Bayesian approach when dealing with the motions of a semisubmersible in a full-scale application, which have not been considered yet?

\subsection{Methodology}

The fulfillment of the objectives enumerated in the previous section results in elaborated and complex procedures. Thus, it is interesting to provide a general idea of the 
approach adopted to meet each one of these topics.

\subsubsection{The non-parametric approach: The Bayesian VMB wave inference}

Since the main interest is the adoption of the Bayesian VMB wave inference approach, it is important to validate the relevance of the hypothesis endorsed in this approach when a semisubmersible platform is adopted as a wave sensor. With this aim, a systematical review of the literature has been carried out. This assessment mainly consists in a comprehensive comparison between the hypothesis in the Bayesian VMB wave inference approach and the theoretical/experimental analysis described in relevant works, such as (GODA, 2000) and (HAVER; MOAN, 1983).

Concerning the sea conditions tested in the wave basin of the TPN-USP, they range from mild to extreme sea states. Thus, the resulting test matrix allows the evaluation of the feasibility of a semisubmersible platform as a wave sensor in a wide range of sea conditions. Moreover, the features of the water surface elevation time series, which have been measured by means of wave probes, are evaluated to determine if they are consistent with the assumptions previously reviewed. It is important to highlight the fact that the adoption of capacitive waves probes to register the water surfaces elevation guarantees accurate registers without disturbing the mechanically generated waves.

This approach ensures that the sea conditions generated during the experimental campaign as well as the model of the semisubmersible paltform are in agreement with the hypotheses adopted to estimate the sea conditions by means of the bayesian VMB wave inference technique.

\subsubsection{The role of the prior distribution in the Bayesian VMB wave inference}

Previous works estimating the sea conditions by means of the Bayesian VMB wave inference approach (e.g. (ISEKI; OHTSU, 2000; NIELSEN, 2005) or (TANNURI et al., 2003)) have in common the model that describes the dynamic responses of the vessel, which linearly relates the excitation (in this case the sea state, that is the unknown of the problem) with the measured motions. However, differences arise between the prior 
distributions presented in the literature. More precisely, these differences appear between the number of the prior parameters adopted to estimate the sea state and the approach followed to estimate their values. In other words, the main difference between these works has its origin in the relevance given to the prior knowledge and the hypothesis adopted regarding the wave spectrum.

A dedicated analysis of these differences have been provided in this thesis. Furthermore, the main conclusions obtained from this assessment are evaluated to determine which prior distribution would be the most appropriate to estimate the sea conditions using the motions of the semisubmersible platform. It is important to remember that this approach envisages the identification of the main shortcomings that characterize each prior distribution, which posteriorly have been taken into account in order to develop a new prior distribution to overcome them.

\subsubsection{Modelling non-linearities of the resonant motions}

It has been pointed out that the techniques based on the wave buoy analogy have in common the approach followed to model the dynamics of the wave sensor, that relates the excitations with the induced responses through a transfer function. Even though the approach adopted in the literature already includes some uncertainties of the model by means of adding a certain amount of noise, it is expected that non-linear effects may result in misleading estimations.

More precisely, the effects of these non-linearities start to become relevant close the resonance of the motions, that corresponds to the frequency range where the damping terms govern the dynamics of the platform. Consequently, the VMB wave inference approach may render inaccurate estimations of the sea conditions for low frequency sea waves, where the resonances of the motions appear.

In order to determine the linear model and also take account of these non-linearities the following approach is followed.

- The transfer functions adopted to model the dynamics of the platform have been obtained in the frequency-domain using a diffraction analysis, which characterizes the primary hydrodynamic parameters of the platform. The resulting transfer func- 
tions can be improved adopting a representative constant damping value for each motion, thus leading to bounded responses of the platform close its resonances. The damping values used in this work have been estimated empirically, using the data obtained during the calibration tests carried out at the wave basin in TPN-USP.

- A sensitive analysis of the possible damping effects arising from the submerged lines (moorings \& risers) has been carried out through numerical simulations in ANSYS-AQWA. These simulations compare the responses of the model without the submerged lines (as it is tested in the wave basin) with the responses of a quasi complete model, that includes the submerged lines (mooring and risers). It is important to remember that due to the nature of the problem being solved, nonlinear effects only have been evaluated for the motions that present their resonance in the wave frequency range of interest.

- A theoretical approach is followed to develop a simple model that estimates the damping effects on the heave motion. This model is based on the hydrodynamic drag forces acting on the pontoons and provides the damping of the motion in accordance with the measured amplitudes. Thus, the response amplitude functions can be upgraded constantly using the amplitude of the motions measured. Also, the reliability of the approach proposed to estimate the external damping has been analyzed by means of a comparison between the results obtained through this methodology and the experimental measurements.

\subsubsection{Assessing the accuracy of the VMB wave inference method- ology based on a semisubmersible platform}

In order to estimate the sea conditions generated in the wave basin in the TPN-USP the measured motions of a model-scale semisubmersible platform have been adopted. These motions are recorded through an optical tracking system, which guarantees accurate recordings of the model motions. Since the responses of all the six degrees of freedom (dofs) have been recorded, the linearity of the responses is assessed by means of the coherence functions to determine the most convenient base of motions for the wave inference.

At the same time, the measurements obtained during the experimental campaign through the wave probes have been used as a paradigm to validate the numerical char- 
acterization of the semisubmersible platform model as well as the estimations obtained through the VMB wave inference.

\subsection{Text structure}

The contents of the thesis have been organized into six chapters, including the present one. Chapter 2 is devoted to a review of the literature concerning the existing motionbased wave inference methodologies. Chapter 3 provides a brief analysis of the stochastic description of the sea states and the hypotheses assumed to compute the motion responses of the platform. The contents of this chapter are also linked to appendices A, B and $\mathrm{C}$, which include a detailed analysis regarding various statistical properties of the sea conditions generated in the wave basin and their extrapolation to real-scale. Moreover, appendix D brings an assessment of the heave responses of a semisubmersible platform. Chapter 4 contains a detailed description of the Bayesian inference approach and the main hypothesis adopted; also, the main inference methods adopted in this work are explained systematically, including the development of an alternative prior distribution for wave inference. The contents of this chapter are associated with appendix E, that aims at providing a broader understanding of the features of the prior distribution. Chapter 5 includes the description of the model-scale experimental campaign carried out in the wave basin of the TPN-USP, using a scaled model of a large semisubmersible platform (content that is related to appendix F). This chapter also presents the results of the estimations obtained using the motion-based Bayesian inference method, that were checked against the measurements obtained by means of an array of wave-probes. Finally, chapter 6 is a summary of the main conclusions reached through this work, that are complemented with recommendations for future work. 


\section{LITERATURE REVIEW}

In general, there are several approaches that may be considered for performing wave inference based on the recorded motions of a vessel (see Nielsen (2017) for a recent review). As a first option, the wave spectrum can be estimated on a parametric approach. This method is used to estimate the sea conditions from directional buoy measurements. The main advantage that this technique has is that it is conceptually simple and it is based on the parametric representation of the directional sea spectrum. Some examples of this method are provided in: (HUA; PALMQUIST, 1994), where the authors adopted a variational method to estimate the optimal values of the spectrum parameters using the full-scale data measurements carried out on a ro/ro ship. Another work based in this approach is presented by Tannuri et al. (2003), who used the data from on-board motion measurements of a moored FPSO and the directional sea spectrum estimations were obtained by means of a parametric model.

The parametric modelling has gained increasing attention in recent years. For example, in (MONTAZERI; NIELSEN, 2014) the authors used the parametric approach to estimate the directional wave spectrum, fitting a standard JONSWAP spectrum, from fullscale response measurements carried out on a container ship. One additional example of the parametric modelling approach can be found in (MONTAZERI; NIELSEN; JENSEN, 2015), which presented a methodology that upgraded the estimation procedure by means of introducing the trends identified in the parameters of the wave spectrum model. These trends were identified in an analysis that was carried out in parallel with the main wave inference estimation routine. In this work the authors obtained good agreement between the estimations and wave radar measurements of the sea state. Finally, (MONTAZERI; NIELSEN; JENSEN, 2016) stands as another recent work in which a parametric model was adopted for estimating wind seas and swells based on measured ship motions. 
Alternatively, another line of works proposes the estimation of the directional wave spectrum based on non-parametric methods. The inference is then made using the wave buoy analogy, which relies on a comparison between measurements of response spectra and calculated ones. (WEBSTER; DILLINGHAM, 1981) is one of the first studies estimating the directional wave spectrum using on-board measured ship motions. In this case the wave spectrum was estimated using a linear programming approach. Thus, the methodology proposed provided accurate estimations in the frequency range where the response amplitude operators of the ship were linear and had some directional dependence. Another example is provided by Hirayama (1987), where the sea spectrum was computed by means of the Maximum Likelihood Method, based on the real-scale measured ship motions. A current example of this approach is provided in (NIELSEN; GALEAZZI; BRODTKORB, 2016), where the authors evaluated a novel concept for wave estimation adopting the wave buoy analogy using model-scale measurements. The approach adopted in this case was formulated directly in the time-domain combining different techniques and the least squares fitting to estimate wave amplitude and phase. The results obtained during the study showed that the statistical parameters were accurately estimated when compared with the measurements obtained through waves probes.

Regarding the directional wave spectrum estimation based on the so-called VMB Bayesian modelling, it was proposed in (ISEKI; OHTSU, 2000), for a ship with forward speed. In order to validate the technique proposed, towing tank experiments and onboard measurements were carried out by the authors. This approach has been adopted also by Nielsen (2006), who presented derivations of both parametric and non-parametric theories. The theories for both methods were applied in order to obtain the directional wave spectrum using the motions of a container ship. The estimations obtained with these methods were compared to measurements made by a wave radar, obtaining good agreement between both approaches. The same researcher carried out a detailed study (NIELSEN, 2007) of numerical simulations of wave records, providing an estimation of the sea state parameters. In his work, two approaches were considered: The parametric procedure and the non-parametric one. The paper finally concluded that it is difficult to favor a particular method since both render similar results. A common feature of the Bayesian wave inference approach as adopted in (ISEKI; OHTSU, 2000), (NIELSEN, 2006) and (NIELSEN, 2007), is that the prior distribution is computed using the Akaike's 
Bayesian Information Criterion (AKAIKE, 1980). However, Iseki (2012) showed that this approach may not provide optimal values for the prior parameters, for sea conditions for which non-linear effects begin to be relevant and, consequently, the uncertainties associated to the response amplitude operators are increased.

Following a similar approach, (SIMOS et al., 2010) presented a complete analysis of the sea states estimated from the motions of a model-scale FPSO. These estimations were performed using the Bayesian inference method with three prior parameters, analogous to the method proposed by Nielsen (2008), but with a relevant conceptual change. Different from the original proposal where the hyperparameters were estimated by means of the ABIC method (TANNURI et al., 2003), the authors set a pre-calibration of these hyperparameters, meaning that their values were prescribed for each wave estimation. From a conceptual point of view, although the mathematical structure of the Bayesian model was preserved, an important feature of the Bayesian approach was abandoned, i.e. the adoption of an information criterion for searching the best calibration of the prior parameters in each estimation event. A detailed description of the procedure followed to calibrate these hyperparameters can be found in (BISPO, 2011a) and in (BISPO, 2011b). This approach led to a much more expedite estimation procedure while the results obtained in the experimental campaign attested that the wave conditions could still be inferred with good accuracy.

Later, Simos et al. (2012) utilized full-scale data measurements from a FPSO operating in Brazil's Campos basin for a first evaluation of the performance of this method in field conditions. However, due to the lack of alternative wave sensors, the comparison of the results was made almost exclusively with hindcast measurements provided by NOAA for the same region where the FPSO was operating. The authors concluded that the method was capable of inferring the sea states with a good agreement with the NOAA measurements, despite the inherent limitations concerning the estimation of sea states with low peak periods. Bispo et al. (2016) provide another feasibility study of the methodology proposed in (SIMOS et al., 2010); for this purpose the first part of the results from a new field campaign, started in December 2014, was presented. Again, results were obtained from full-scale FPSO motions using the Bayesian inference approach with three pre-calibrated hyperparameters. The performance of the VMB wave inference method is checked against the wave estimations provided by a commercial marine radar 
system. Comparison between both systems attest an adequate identification of the wave conditions.

Assessing the feasibility of a semisubmersible platform as a motion based wave sensor is the main general objective of this thesis. This topic was first investigated by Wijaya (2009). In order to validate the hypothesis that a semisubmersible platform can be used as a VMB wave sensor, his work was mostly based on model-scale tests of the Åsgard-B semisubmersible platform, which has also been adopted as a case-study for this thesis. The tests assessed in Wijaya (2009) were conducted in the Marintek facilities with the model subjected to waves, wind and currents. Since the experimental campaign was carried out under combined environmental conditions (waves, wind and current), the model of the platform included the submerged lines (riser and moorings) as well the topside. Nevertheless, only a short range of wave conditions was evaluated due to the fact that the analysis was based on a set of final design tests. The author also assessed some full-scale results but these were limited because concerned a short period of time and only included mild wave conditions. With regard to the modelling approach followed, the estimation of the sea spectrum was obtained using the parametric modelling. In order to properly evaluate the accuracy of the results obtained with this inference approach, model-scale results were compared to measurements obtained in the wave basin. Measurements from wave radar and buoy were adopted as the paradigm for the full-scale estimations. In general, the author concluded that the results, for model-scale and real-scale, shown good agreement with the measurements of the significant wave height. Nevertheless, the author pointed out that significant discrepancies regarding the mean direction and peak period were noticeable. Also, some deviations (over-estimation) can be observed for the inferred values of the significant wave height. Finally, as a consequence of the discrepancies reported between the estimations and the measurements, the author concluded that further research should be conducted in order to determine if a semisubmersible platform can be adopted as a VMB wave sensor.

In the present study, a scaled model of the Åsgard-B semisubmersible unit was built, and tested in a large set of experimental conditions at the wave basin in the TPN-USP wave basin. They were specifically conceived for verifying the feasibility of performing the VMB wave inference estimations, putting a special emphasis on severe wave conditions, and its frequency range limitations when this type of offshore platforms is adopted as a 
wave sensor.

On the other hand, further attention should be paid to the mathematical basis of the problem, which, in principle, consists of an inverse problem in which the input (sea spectrum) has to be estimated from the measured output data (floater motions), by means of the Bayesian modelling approach. The conventional methodology adopted in the works described above does not include the inherent uncertainties in the estimates of the ship response functions. A first step in this attempt was made by Iseki (2012), who addressed this challenge with an error matrix, composed of products of the response functions and the estimation errors, achieving a modified Gaussian stochastic linear regression model by the summation of the RAO matrix and the error matrix $(C)$.

Full-scale ship motion data was used to validate the modified Bayesian procedure proposed, and Iseki (2012) showed that this alternative approach provides improved estimations of the different sea conditions and increases the reliability of the hyperparameters estimated by means of the ABIC.

This thesis includes several references to other wave monitoring systems, such as buoys and marine radar systems. Although the study and characterization of these systems are not in the scope of this work, a brief review is needed, regarding the mathematical models commonly adopted and their main drawbacks. Traditionally, wave measurements have been carried out by means of directional wave buoys. These are floating devices equipped with different measuring systems (including accelerometers and inclinometers). Thus, wave measurements can be obtained using the recorded signals of the measuring systems. Usually, the approach followed to estimate the sea spectrum characteristics is the parametrical modelling, see for example (HASSELMANN; DUNCKEL; EWING, 1980) or (ALLENDER et al., 1989). The possible drawbacks of this measurement system have been described in chapter 1 . In addition to directional wave buoys, wave radar systems have gained notorious popularity as a wave monitoring system. In general, they provide accurate measurements and information about the sea spectrum in a wide frequency range. However, in extreme sea conditions, as it has been pointed out before, these systems may present different shortcomings (MAGNUSSON, 2008) that generate imprecise measurements of the wave heights and the energy distribution. One may realize that both systems have a common shortcoming regarding the inaccurate estimations of extreme sea 
conditions, that leads to divergent inferred values for the same sea conditions. Accordingly, the recover of reliable estimations of the sea spectrum characteristics in extreme sea states is one of the main motivations to use a large semisubmersible platform as a VMB wave sensor, as discussed by Wijaya (2009).

Finally, it should be stressed that this section has not included a detailed review of the works related to the Bayesian inference approach; this is due to the fact that the most prominent literature references have been included in the bulk text of section 4, where the method fundamentals are also covered. 


\section{LINEAR MODELLING OF OCEAN WAVES AND WAVE INDUCED RESPONSES OF FLOATING OFFSHORE STRUCTURES}

\subsection{Introduction}

Since the methodology investigated in the present thesis aims to determine the sea conditions based on the platform motions, the accuracy of the output is associated with the hypotheses and simplifications regarding the wave description and the responses of the floating platform. In particular, this technique is strongly sensitive to the precision of the model adopted to estimate the vessel responses. For this reason, this chapter aims at providing a brief review of the hypotheses and properties related to the description of ocean waves and the induced responses of the vessel.

\subsection{Description of ocean waves}

The proper description of ocean waves is of interest to the dynamics of ships and offshore structures. In normal conditions the sea surface comprises a large number of waves with different lengths, heights and directions. As a direct result of this heterogeneity, the water surface elevation cannot be described by means of a deterministic mathematical expression. A common approach adopted to describe the sea surface elevation is to assume that it is given by the superposition of many simple, regular wave components, each one with its own amplitude, length, frequency and direction of propagation. This concept (also known as the superposition principle) was first adopted in (DENIS; PIERSON, 1953) to describe the behavior of the sea surface in terms of a simple theory of regular waves.

Regarding the characterization of regular waves, it is based on major waves theories, such as Airy linear wave theory, that were developed during the $19^{\text {th }}$ century. The re- 
sults of these theories are also of central importance for solving the seakeeping problem. Therefore, the most relevant definitions and theory used to describe the regular waves are discussed in the following section. In this case, due to the fact that only a brief review is provided, the reader is referred to (NEWMAN, 1977) for a detailed and systematical description of the theory and formulae.

\subsubsection{Regular waves}

Figure 3.1 shows the main features and characteristics adopted to define the regular waves. As a matter of fact, this figure provides the wave profile, $\eta$, accordingly to the distance, $x$, for a given instant of time, $t$. It is worth remembering that the coordinate system is placed at the still water level (SWL) with the y-axis directed upward and the $\mathrm{x}$-axis is positive in the direction of wave propagation.

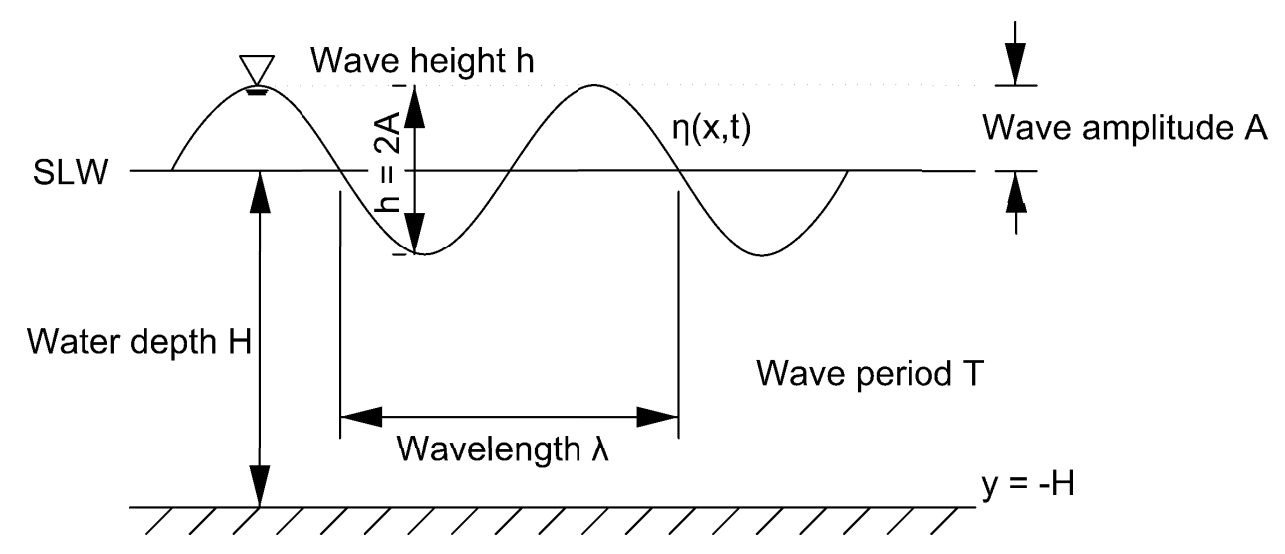

Figure 3.1: Harmonic wave definitions.

Even though the regular wave constitutes the simplest component of an irregular sea, some assumptions are still needed to develop a theory that describes it. In the following, the assumptions in which this approach is based are provided.

1. The flow is assumed to be 2-dimensional;

2. The flow can be described by the velocity potential, $\phi$;

3. The flow can be considered as irrotational flow, i.e. vorticity $\nabla \times \mathbf{u}=0$. It is important to remember that this condition does not apply where flow separation occurs; 
4. Fluid motion in the ocean can be assumed as a potential flow, which is formally characterized by:

- The velocity can be described in terms of a scalar potential,

$$
\mathbf{u}(x, y, z, t)=\nabla \phi(x, y, z, t)
$$

- The velocity potential must fulfil the following form of the continuity equation, also known as the Laplace equation, given by,

$$
\nabla^{2} \phi=0
$$

- The resulting momentum equation is,

$$
\frac{p(x, y, z, t)}{\rho}=-\frac{\partial \phi}{\partial t}-\frac{1}{2}|\nabla \phi|^{2}-g y .
$$

where $p$ is the pressure and $\rho$ and $g$ stand for the density of the water and the gravity constants, respectively.

5. Small wave amplitudes are assumed: This hypothesis is equivalent to state that $\frac{A}{\lambda}<<1$. Thus, resulting in $\frac{\phi}{\lambda^{2} T}<<1$.

Therefore, the problem is reduced to solve the Laplace equation for the velocity potential, given certain boundary conditions (BCs). This linear description of the propagation gravity waves is commonly known as the linearized (Airy) wave theory.

Regarding the BCs, deep water condition is assumed ${ }^{1}$, that is equivalent to set $\nabla \phi \rightarrow$ 0 , for $y \rightarrow-\infty$. This condition has been adopted due to the fact that it results in a simple solution of the velocity potential, which equally illustrates the main assumptions and simplifications adopted in this theory. Again, (NEWMAN, 1977) is provided as the main reference for a detailed review of the problem including the $\mathrm{BC}$ for $y=-H$.

Now, it is necessary to describe the behavior of the free surface. For this purpose two different BCs are endorsed:

1. Kinematic BC: It is assumed that the normal component of water velocity at water surface is the same as the normal component of the velocity of the surface. In other

\footnotetext{
${ }^{1}$ In the linear wave theory the water conditions are considered to be deep if the water depth, $H$, is more than half the wave length, $\lambda$, so: $\frac{H}{\lambda}>\frac{1}{2}$ or $\frac{\lambda}{H}<2$.
} 
words, this $\mathrm{BC}$ is equivalent to accept that water particles at the surface stay on the free surface and that the flow has to be tangential at the free surface. Formally, this condition is given by,

$$
\frac{\partial \eta}{\partial t}=\frac{\partial \phi}{\partial y}-\mathbf{u} \cdot \nabla \eta .
$$

2. Dynamic BC: The pressure on the both sides of the surface is equal. Which is equivalent to assume that in the free surface the hydrodynamic pressure $(p)$ equals the atmospheric pressure $\left(p_{a}\right)$. This relation can be expressed by means of the momentum equation,

$$
-\frac{1}{\rho}\left(p-p_{a}\right)=\frac{\partial \phi}{\partial t}+\frac{1}{2}[\nabla \phi \cdot \nabla \phi]+g \eta=0,
$$

Now, in virtue of the small amplitude assumption $\left(\frac{A}{\lambda}<<1\right)$, it can be shown that slopes of the wave profile, i.e. $\frac{\partial \eta}{\partial x}$, are small quantities compared to one. Moreover, the derivatives of the velocity potential are small first order-quantities, as a consequence of the fact that the fluid velocity can be assumed as a small quantity proportional to the wave motion. Therefore, the non-linear terms in Eqs. (3.4) and (3.5) can be neglected. In such conditions, the resulting formal expressions of the linearized BCs are,

$$
\begin{gathered}
\frac{\partial \eta}{\partial t}=\frac{\partial \phi}{\partial y} . \\
\eta=-\frac{1 \partial \phi}{g} \frac{\partial t}{\partial t}
\end{gathered}
$$

These BCs can be presented together in a more elegant form. Thus, combining Eq. (3.6) with Eq. (3.7),

$$
\frac{\partial^{2} \phi}{\partial t^{2}}+g \frac{\partial \phi}{\partial y}=0
$$

Before taking any further steps, it is important to highlight that the resulting BCs 
adopted to describe the behavior of the sea surface are characterized by two linearizing hypothesis: (i) non-linear terms of the dynamic BC have been omitted (thus, highlighting the importance of the hypothesis of small wave amplitudes) and (ii) the BCs are applied at $y=0(\mathrm{SWL})$ and not to $y=\eta(x, t)$.

The straightforward velocity potential that fulfils the Laplace equation as well as the BCs described is given by,

$$
\phi=\frac{g A}{\omega} e^{k y} \sin (k x-\omega t)
$$

where $k$ stands for the wave number and $\omega$ for the wave frequency. Thus, the general form of the free surface elevation can be expressed as,

$$
\eta(x, t)=A \cos (k x-\omega t)
$$

As it was stated previously, the relevance of this theory is well illustrated by the principle adopted to describe the irregular waves, which allows the representation of irregular times series of the sea surface elevation as the result of the linear superposition of many (regular) waves, each one with different phase shift, $\phi_{i}$, amplitude and frequency. This linearization can be formally expressed as,

$$
\eta(x, t)=\sum_{i=1}^{N} \eta_{i}=\sum_{i=1}^{N} A_{i} \cos \left(k_{i} x-\omega_{i} t+\phi_{i}\right) .
$$

where each $\eta_{i}$ is derived by means of combining Eq. (3.7) and (3.9). For instance, the regular waves included in Eq. (3.11) can be characterized using the theory discussed in this section. A detailed review of the main methodology followed to characterize the ocean surface elevation is provided in the following.

\subsubsection{Irregular waves}

The reader must be already aware of the fact that the description of the ocean waves is a topic of major significance for the purpose of this thesis. An analysis regarding the background of the regular waves has been provided in the previous section. This methodology, however, does not characterize the sea surface elevation by itself. Altogether, it 
has been discussed that the sea surface is a non-deterministic process in space and time, thus the probabilistic approach is commonly adopted in order to describe its main characteristics. The major fundamentals of the approach discussed hereafter are thoroughly described in the literature, e.g. (BISHOP; PRICE, 1979) or (GODA, 2000).

In order to obtain a realistic idea of the wave forces acting on offshore structures, the sea surface elevation is considered as a time-space dependent random process (GODA, 2000). An illustration of the statistical characteristics of this process has been provided in appendix A, which consists of an analysis of a sinusoidal wave as a random process. However, the characterization of a random process is relatively complex. Considering the properties discussed in appendix A, the sea surface elevation can be assumed as a stationary, homogeneous and ergodic random process ${ }^{2}$. As a result of these hypotheses, the probability density function of the surface elevation is the same for different records, given that the statistical properties remain constant for any (sufficiently long) time span and location.

These assumptions make it possible to characterize the sea condition from a single wave record. The common approach is the use of power spectral density (PSD) estimators, which are based on the Fast Fourier Transform (FFT) of the wave record and that are in accordance with the model described in Eq. (3.11). In sea wave analysis, the use of the FFT stands as the most practical way to estimate the PSD, providing good frequency resolution. However, the ideal method should provide high resolution regarding rapid temporal changes in analytic state variables for frequency, phase and amplitude. These characteristics of the analysis can be obtained, for example, by means of the Hilbert Transform. It has to be said, however, that both methods give similar results when applied to signals having relatively long durations, as is the case in sea wave analysis. As a consequence of the similarity of the outputs when applied to signals that are relatively long and for practical purposes, in this thesis the power spectral density estimations will be computed using models based on the FFT of the measurements.

The description of the wave energy distribution over the frequency and direction ranges is provided by the directional energy wave density spectrum, $S_{\eta \eta}(\omega, \beta)$, which stands as an statistical characteristic of the sea. Then, the energy density (per unit area

\footnotetext{
${ }^{2}$ Appendix B also provides a broad discussion regarding these ideas.
} 
of the sea surface) of a given sea state within these ranges can be computed from this spectrum as follows,

$$
E=\rho g \int_{0}^{2 \pi} \int_{-\infty}^{+\infty} S_{\eta \eta}(\omega, \beta) \mathrm{d} \omega \mathrm{d} \beta
$$

Returning to the Fourier analysis, it widely known that it allows the description of the surface elevation as the sum of infinite waves for all possible combinations of frequencies in $N_{k}$ directions, leading to the Longuet-Higgins model (see, once again, appendix A) which is similar to the one presented in Eq. (3.11) to describe the irregular wave elevation,

$$
\eta(\mathbf{x}, t)=\sum_{j=1}^{N_{k}} \sum_{i=1}^{N \longrightarrow \infty} A_{j i} \cos \left(\mathbf{k}_{j i} \cdot \mathbf{x}-\omega_{i} t+\phi_{i}\right),
$$

where the wave number vector $\mathbf{k}_{j i}=k_{i}\left(\cos \left(\beta_{j}\right), \sin \left(\beta_{j}\right)\right)$ has magnitude $k_{i}$ and the randomness of the process is obtained by means of random phases, $\phi_{i}$. Therefore, making use of the central limit theorem, it is assumed that the sea surface elevation has a normal distribution.

Accordingly, the energy density can be computed as the sum of the energy of the wave components of Eq. (3.13),

$$
E=\rho g \int_{0}^{2 \pi} \int_{0}^{+\infty} S_{\eta \eta}(\omega, \beta) \mathrm{d} \omega \mathrm{d} \beta=\rho g \int_{0}^{2 \pi} \int_{0}^{+\infty} \frac{A^{2}(\omega, \beta)}{2} \mathrm{~d} \omega \mathrm{d} \beta,
$$

which provides a relation between the sea energy spectrum and the amplitudes of the waves that compose it. Thus, the following equivalence exists between $S_{\eta \eta}(\omega, \beta)$ and the amplitudes of the individual regular waves,

$$
S_{\eta \eta}(\omega, \beta) \mathrm{d} \omega \mathrm{d} \beta=\sum_{\beta}^{\beta+\mathrm{d} \beta} \sum_{\omega}^{\omega+\mathrm{d} \omega} \frac{A^{2}(\omega, \beta)}{2}
$$

The assumptions adopted regarding the sea surface (it is stationary, narrow banded and Gaussian distributed) allow the adoption of the Rayleigh distribution to characterize the probability of the wave amplitude. A comprehensive discussion of the Rayleigh distribution and how it is related to the Normal probability distribution function is provided 
in (BENDAT; PIERSOL, 2010) $)^{3}$.

As it has been pointed out earlier, the spectral density function allows the statistical characterization of any sea state and it is usually described in a parametric way based on some statistical parameters of the waves. Some common ones are provided herein.

\footnotetext{
${ }^{3}$ This model is correct when the number of waves components tends to infinity (see Goda (2000)). However, a common practice is to obtain the sea conditions using a finite number of sinusoidal waves, producing series that have their variance bounded and, consequently, that are neither Gaussian nor ergodic (TUCKER; CHALLENOR; CARTER, 1984). Notwithstanding this, it is widely known that the statistical distribution of the individual wave heights is well approximated by a Rayleigh distribution. Thus, herein an analysis is provided, regarding the expected distribution of $S_{\eta \eta}\left(f_{i}\right)=S_{\eta \eta_{i}}$ and its variance, when the number of components in the Longuet-Higgins model tends to infinity.

Let $s_{\eta \eta_{i}}:=\frac{1}{2} A^{2}=S_{\eta \eta}\left(f_{i}\right) \Delta f_{i}$. Also, it is now known that $A$ is Rayleigh distributed, thus its probability density function can be written as,

$$
\operatorname{pdf}(A)=\frac{A}{\sigma^{2}} \exp \left(-\frac{A^{2}}{2 \sigma^{2}}\right),
$$
}

The change-of-variable technique can be used as follows,

$$
\operatorname{pdf}(A) \mathrm{d} A=\operatorname{pdf}\left(s_{\eta \eta_{i}}\right) \mathrm{d} s_{\eta \eta_{i}}, \quad \text { where } \frac{\mathrm{d} A}{\mathrm{~d} s_{\eta \eta_{i}}}=\frac{1}{A},
$$

Leading to,

$$
\operatorname{pdf}\left(s_{\eta \eta_{i}}\right)=\frac{1}{\sigma^{2}} \exp \left(-\frac{s_{\eta \eta_{i}}}{\sigma^{2}}\right)
$$

Therefore, $s_{\eta \eta_{i}}$ has an exponential distribution. Regarding the variance, following (OCHI, 1990) it can be rewritten as,

$$
\operatorname{Var}\left(s_{\eta \eta_{i}}\right)=E\left[\left(s_{\eta \eta_{i}}\right)^{2}\right]-E^{2}\left[s_{\eta \eta_{i}}\right]=\sigma^{4}
$$

Thus the coefficient of variation is,

$$
\operatorname{CoV}\left(s_{\eta \eta_{i}}\right)=\left(\frac{\operatorname{Var}\left(s_{\eta \eta_{i}}\right)}{E^{2}\left[s_{\eta \eta_{i}}\right]}\right)^{\frac{1}{2}}=1,
$$

Meaning that the standard deviation of an exponential distribution is equal to its mean. Finally, if the spectrum, $S_{\eta \eta}$, is estimated from a real Gaussian process,

$$
E\left[S_{\eta \eta_{i}}\right]=\frac{\sigma^{2}}{\Delta f}=S_{\eta \eta_{i}} .
$$

where $S_{\eta \eta_{i}}$ is the averaged spectrum of the spectra, $S$, for the $i^{t h}$ frequency, obtained for each realization, $\eta(t)$, of the same process.

But, in this ideal condition the coefficient of variation is $1\left(\operatorname{CoV}\left(S_{\eta \eta_{i}}\right)=1\right)$, for a $\Delta f=$ const.

For the analysis of practical (time-limited) wave records this quantity provides a measurement of the expected variance of each component of the spectrum. In order to have a clear idea of the origin of this variance a brief explanation is provided. A random process can be featured by a continuous spectrum, in other words, the measured signal (recorded over a extremely long period of time) is the result of the sum of a large number of components closely spaced in frequency and with random phases. If a short record of the signal is now taken, each harmonic of the spectrum splits its energy amongst the adjacent components of the spectrum, as described in (SHIN; HAMMOND, 2008). Therefore, each harmonic is given by a large number of contributions which are random in phase. Thus, the amplitude of a particular harmonic is to some extent random. Consequently, the analysis of different records of the same signal is expected to result in a fluctuation of the amplitude of the harmonics from analysis to analysis. This 


\section{Significant Wave Height}

The significant wave height or the mean value of the highest third of the waves, is computed as provided in Eq. (3.16).

$$
H_{s}=3 \int_{\frac{1}{3}}^{+\infty} \frac{h^{2}}{4 m_{0}} \exp \left(\frac{h^{2}}{8 m_{0}}\right) \mathrm{d} h=4.0043\left(m_{0}\right)^{\frac{1}{2}} \approx 4\left(m_{0}\right)^{\frac{1}{2}},
$$

where the variance is computed as $\sigma^{2}=m_{0}=\int_{0}^{2 \pi} \int_{0}^{+\infty} S_{\eta \eta}(\omega, \beta) \mathrm{d} \omega \mathrm{d} \beta$, moment of order zero $^{4}$ of $S_{\eta \eta}$. Traditionally, the significant wave height has been adopted as a typical measure of the energy of the sea spectrum. However, this quantity, by itself, does not provide information regarding the energy distribution in the frequency range.

Such distribution is described by means of statistical wave periods. Most common periods are:

\section{Peak Period}

This period, $T_{p}$, is the wave period with the highest energy.

\section{Mean Wave Period}

The mean wave period, $T_{1}$, is defined as the mean value of all wave periods in a wave record representing a certain sea state. This period is associated to the frequency that corresponds to the spectral centroid of the wave spectrum and it can be estimated as,

$$
T_{1}=2 \pi \frac{m_{0}}{m_{1}}
$$

where the first spectral moment of $S_{\eta \eta}$ is given by $m_{1}=\int_{0}^{2 \pi} \int_{0}^{+\infty} \omega S_{\eta \eta}(\omega, \beta) \mathrm{d} \omega \mathrm{d} \beta$.

\section{Mean Zero Up-Crossing Period}

Additionally, the expected value of the zero up-crossing period stands for the average period of zero-crossing waves. It can be associated to the frequency that represents the radius of gyration around $\omega=0$ of the area under the spectrum and its value is computed by means of the zeroth and second order spectral moments as shown below,

result will be important to understand the Gaussian hypothesis and its implications.

${ }^{4}$ Regarding the moment of order $n^{t h}$, it can be computed as $m_{n}=\int_{0}^{2 \pi} \int_{0}^{+\infty} \omega^{n} S_{\eta \eta}(\omega, \beta) \mathrm{d} \omega \mathrm{d} \beta$. 


$$
T_{2}=2 \pi\left(\frac{m_{0}}{m_{2}}\right)^{\frac{1}{2}}
$$

Figure 3.2 provides an illustration of the wave spectrum (in the period domain) and some of the statistical parameters discussed, i.e. the Peak period, Mean wave period and Mean zero up-crossing period.

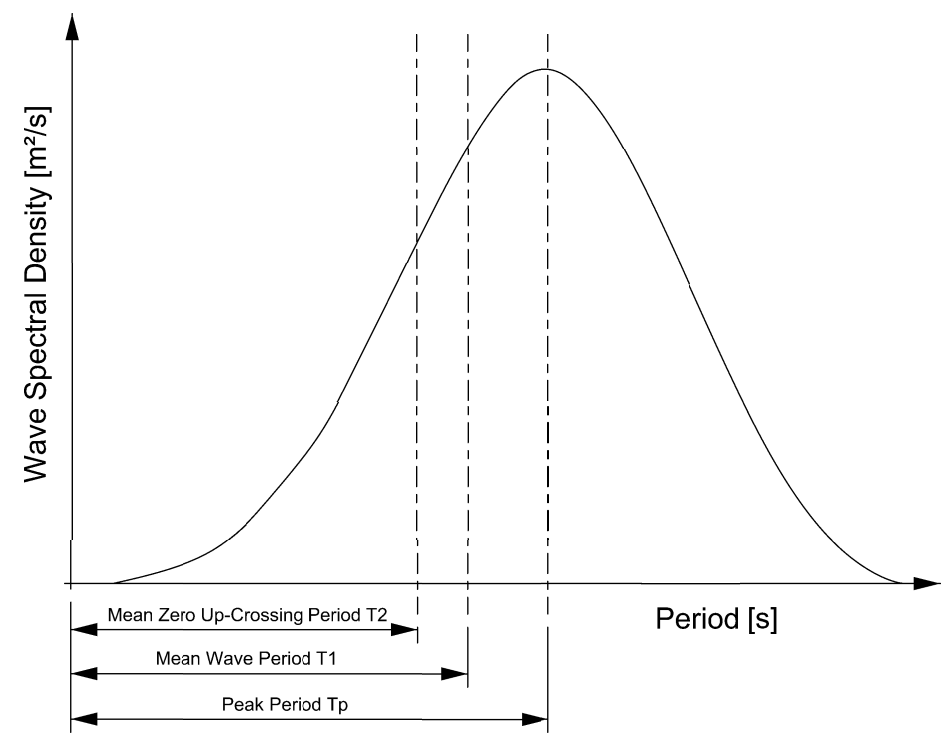

Figure 3.2: Illustration of the Peak period, Mean wave period and Mean zero up-crossing period.

In section 1.1.2, it has been pointed out that the vessel may be intended as a kind of low-pass filter, filtering the wave components that do not excite the vessels responses. This results in one of the major limitations that the VMB wave inference method presents. Consequently, the mean wave period has been used in different works as a measure of the accuracy of the estimation, e.g. (SIMOS et al., 2010, 2012) and (BISPO et al., 2016). Unlike $T_{p}$, this statistical parameter is more sensitive to the energies placed in the high frequency range and allows an accurate assessment of the inferred sea conditions.

\subsubsection{Note on standard wave spectrum models}

The sea states are generally characterized by their PSD. A common approach is to adopt a standard wave spectrum model among the ones that are used to represent the power spectral density in the frequency-domain, $S_{\eta \eta}(\omega)$. There are various idealized spectra that are used nowadays in oceanography and ocean engineering. Perhaps the simplest 
is that proposed in (PIERSON; WILLARD; MOSKOWITZ, 1964), also known as the Pierson-Moskowitz (PM) wave spectrum. Other commonly adopted wave spectra are the Bretschneider (BS) wave spectrum (BRETSCHNEIDER, 1959) and the JONSWAP (HASSELMANN; DUNCKEL; EWING, 1980) wave spectrum. Furthermore, some of these standard wave spectra allow the adjustment of the wave energy distribution through the bandwidth by means of different parameters. Thus, leading to sea spectra that present broader or narrower energy distribution of the ocean waves in accordance with the development stage that sea being modelled presents.

Regarding the directional wave spectrum, $S_{\eta \eta}(\omega, \beta)$, it can be obtained by multiplying the PSD, $S_{\eta \eta}(\omega)$, by a directional spread function, $D(\omega, \beta)$. This functional represents the directional distribution of wave energy. As an example, the Mitsuyasu type directional spreading function is provided next (GODA, 2000). Mathematically, it is given by,

$$
\begin{gathered}
S_{\eta \eta}(\omega, \beta)=S_{\eta \eta}(\omega) D(\omega, \beta), \\
D(\omega, \beta)=G(s) \cos ^{2 s}\left(\frac{\beta-\beta_{0}}{2}\right) .
\end{gathered}
$$

where the parameter $s$ determines the directional spread of the spectrum and $\beta_{0}$ stands as the main direction. The function $G(s)$ is only a normalizing factor used to guarantee an unitary integral, thus, it must fulfil the following condition,

$$
G(s) \rightarrow \int_{0}^{2 \pi} D(\omega, \beta) \mathrm{d} \beta=1 .
$$

For illustrative purposes, Fig. 3.3 provides an example for the directional spread function and the PSD. The combination of this spread function and a JONSWAP wave spectrum (with $H_{s}=1 \mathrm{~m}$ and $T_{p}=14 \mathrm{~s}$ ) leads to the directional wave spectrum shown in Fig. 3.4. It is worth to remember that knowledge of the directional distribution of the energy of sea waves is still limited because of the difficulty in making reliable field measurements and, as a consequence, the establishment of a standard functional form for the directional wave spectrum has not been achieved yet, in contrast to the case of the PSD, $S_{\eta \eta}(\omega)$. Therefore, the functionals commonly adopted to model the directional energy 
distribution are adapted to a limited database of measurements and basically consider a smooth distribution of the energy, including different parameters to set how wide the peak of the wave spectrum must be.
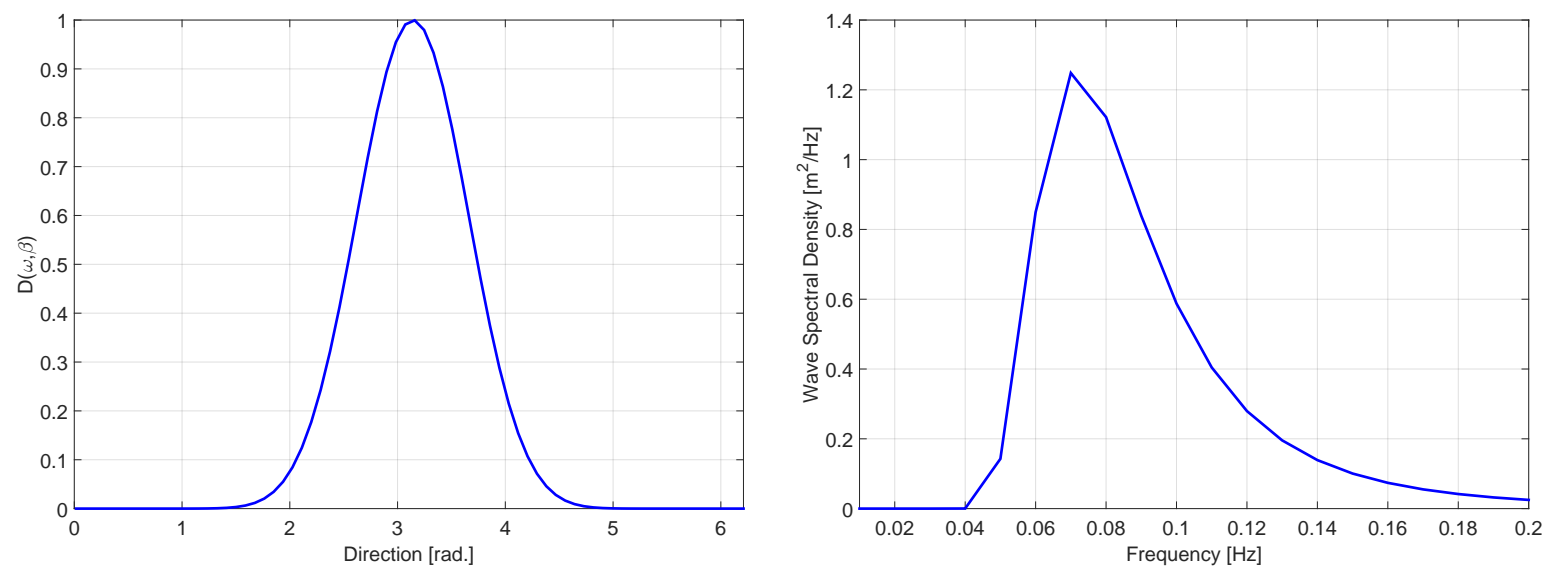

Figure 3.3: Example of cosine-squared directional spread function (left) and the power spectral density (right).

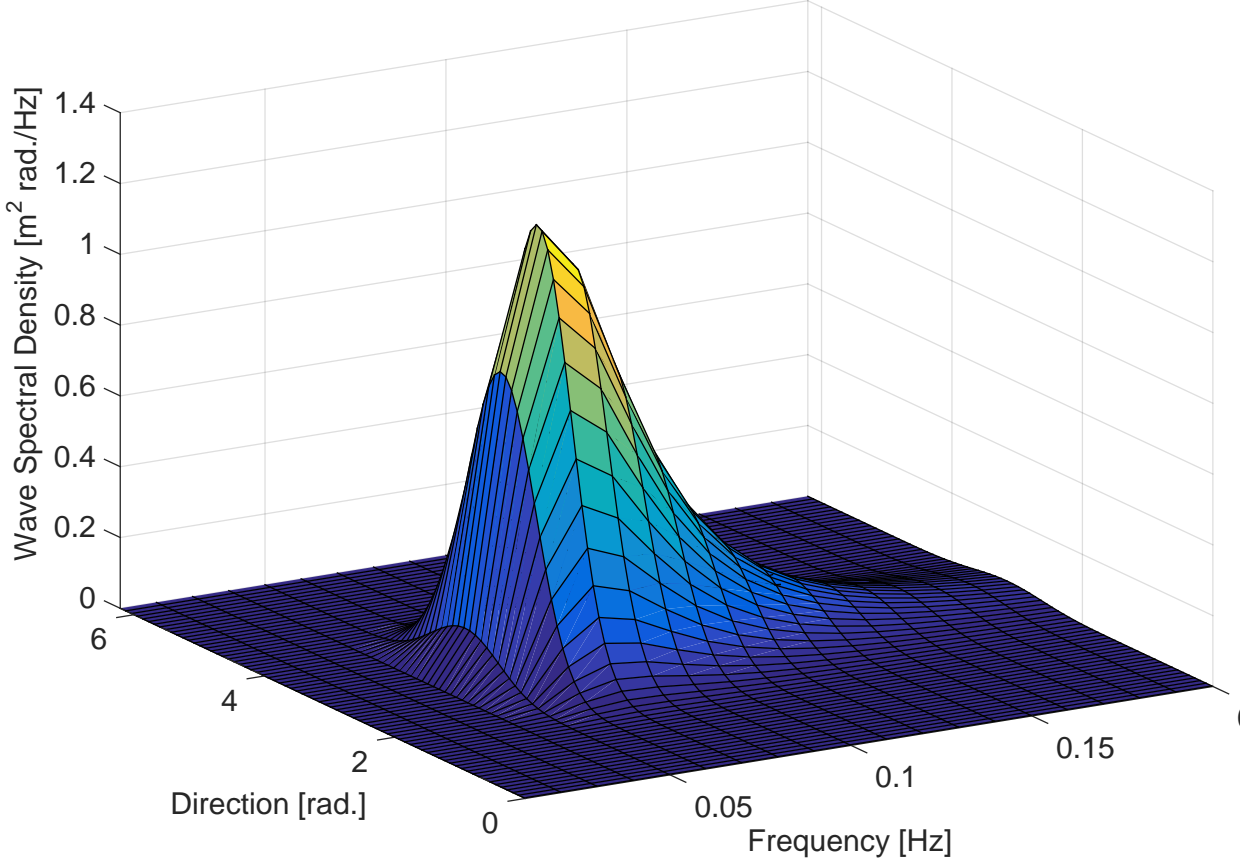

Figure 3.4: Example of directional energy spectrum.

The approach described above reflects the methodology adopted traditionally to characterize the sea states, resulting in directional wave spectra that are regarded as unimodal (with energy concentrated mainly on the wind direction). However, field measurements 
have already demonstrated that the energy of short waves tends to be accumulated along two or more off-wind directions, generating double (or multiple) directional distributions. Several works, e.g. (OCHI; HUBBLE, 1977; GARCIA-GABIN, 2015; TOFFOLI et al., 2010) and (TORSETHAUGEN; HAVER, 2004), provide a detailed discussion and characterization of these bimodal directional wave spectra. Accordingly, some spectral models have been developed to give a realistic approach for double peak cases in the frequencydomain, such as the bimodal spectral model formulated in (OCHI; HUBBLE, 1977) (which is an extension of the BS wave spectrum) and the model developed in (TORSETHAUGEN, 1993), which are referred as Ochi-Hubble and Torsethaugen wave spectrum repectively.

In this thesis, the assessment of the use of a semisubmersible platform as a motion based wave sensor has been carried out using a scale model of the Åsgard-B semisubmersible platform (see section 1.1.2). Therefore, aiming at generating compelling results, the sea spectrum models adopted to generate the irregular sea in the wave basin correspond to: The JONSWAP and Torsethaugen wave spectra, which stand as the most representative wave spectrum models for the sea condition observed in the Norwegian continental shelf. Although these spectra are widely documented in the literature, a succinct description of their formulae is provided in appendix C.

Moreover, Fig. 3.5 shows a comparison between the Torsethaugen spectrum and JONSWAP spectrum for the same sea condition, with significant wave height equal to $1 \mathrm{~m}$ and spectral peak period of $12.6 \mathrm{~s}$. From this figure, one may realize that the main difference between these spectrum models is the energy distribution along the wave frequency axis. On the one hand, the energy is concentrated near the peak of the spectrum in the JONSWAP model and less on frequencies further away from the peak. On the other hand, Torsethaugen spectrum is a bimodal sea spectrum composed by a swell (energy around the peak in the low frequency range) and locally generated wind waves (energy components given in the high frequency range).

In order to illustrate the quantitative differences between the JONSWAP and the Torsethaugen spectrum Tab. 3.1 provides some statistical characteristics of these spectra for different sea conditions. In this case, the Torsethaugen characteristics have been normalized by the correspondent statistical features of the JONSWAP wave spectrum, 


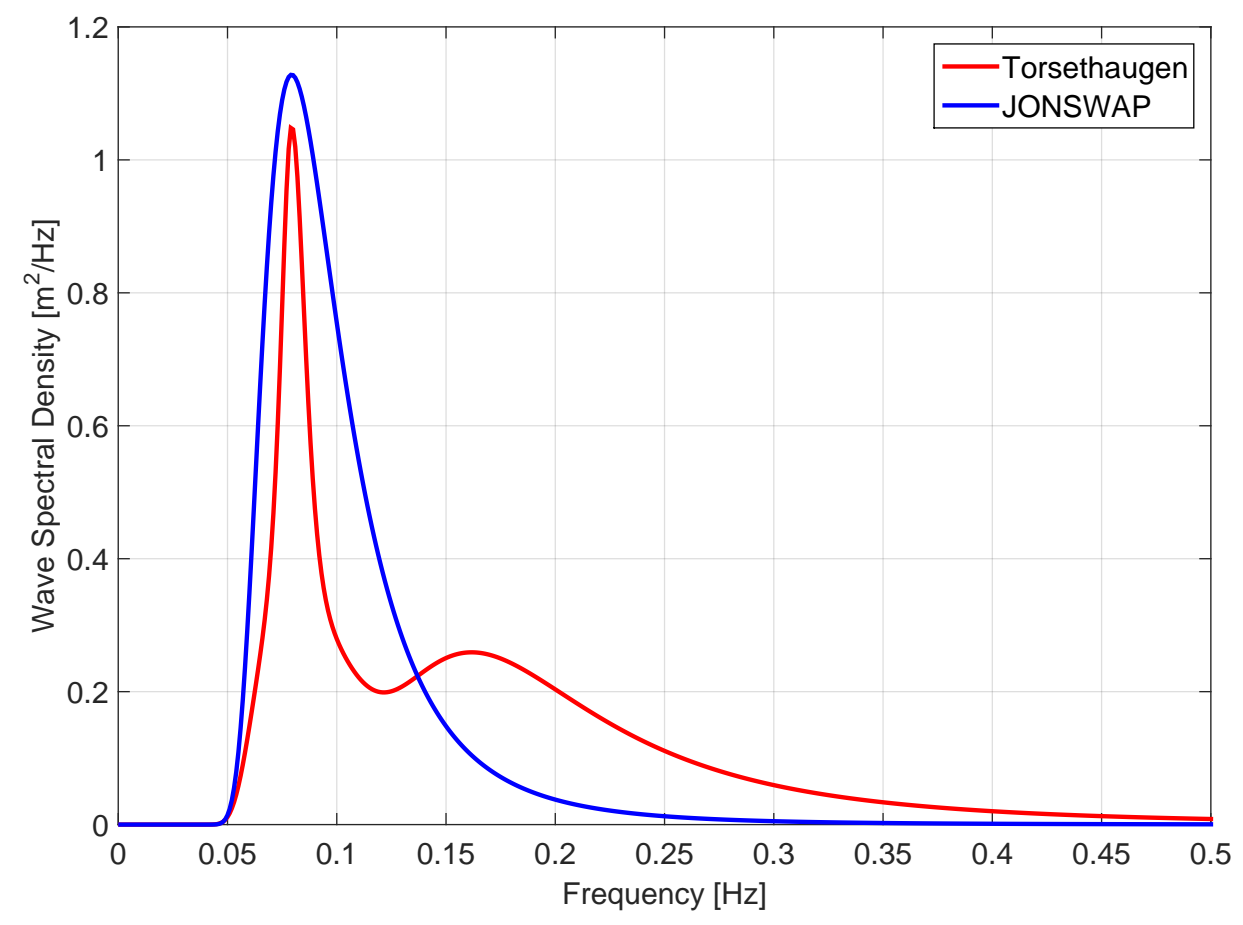

Figure 3.5: Wave spectrum models comparison.

for the same sea condition. The statistics provided include the variance of the wave train given by the area under the spectrum $\left(m_{0}\right)$, the mean wave period $\left(T_{1}\right)$ and the median wave period, or $T_{\frac{1}{2}}$. Concerning the median wave period, it is related to the frequency that exactly divides the spectrum into two equal parts (in the frequency-domain). Therefore, this period also provides meaningful information of the energy distribution being, at the same time, less sensitive to the extreme values than $T_{1}$. The period, $T_{\frac{1}{2}}$, is given by,

$$
\frac{m_{0}}{2}=\int_{0}^{f_{\frac{1}{2}}} S_{\eta \eta}(\omega) \mathrm{d} \omega, \quad \text { where } \quad T_{\frac{1}{2}}=\frac{1}{f_{\frac{1}{2}}} .
$$

It is worth remembering that in this thesis the area calculation for each wave spectrum has been done by integrating the wave spectrum curve by means of a trapezoidal method.

The results provided in Tab. 3.1 show that JONSWAP wave spectrum has a slightly smaller area if compared with the Torsethaugen spectrum. Such differences have also been reported in other works, e.g. (TORSETHAUGEN; HAVER, 2004) and (WIJAYA, 2009). The origin of these differences may be explained, for moderate and high sea states, by the fact that the high frequency tail of the simplified Torsethaugen spectrum model seems to fall off slower (it is proportional to the -4 exponential) than the reference spec- 
trum. Therefore, for the simplified model of the Torsethaugen spectrum proposed in (TORSETHAUGEN; HAVER, 2004), narrow differences between the outputs are considered acceptable when the same sea condition is modelled through different wave spectrum models. Regarding the mean wave period and the median wave period, Torsethaugen spectrum always provides lower values than JONSWAP, as expected, due to the fact that the energy is usually concentrated in the high frequency range. Finally, another feature of the statistics provided is the fact that median period is always greater than the mean period, as expected.

Table 3.1: Wave spectrum properties

\begin{tabular}{cccccccc}
\cline { 3 - 7 }$H_{s}$ & & \multicolumn{3}{c|}{ JONSWAP } & \multicolumn{3}{c}{ Torsethaugen } \\
\cline { 3 - 8 } & $T_{p}\left(m^{2}\right)$ & $T_{1}(s)$ & $T_{\frac{1}{2}}(s)$ & $m_{0}^{*}$ & $T_{1}^{*}$ & $T_{\frac{1}{2}}^{*}$ \\
\hline 1.00 & 12.60 & 0.06 & 9.73 & 10.83 & 1.01 & 0.61 & 0.64 \\
3.50 & 13.50 & 0.06 & 10.42 & 11.63 & 1.02 & 0.79 & 0.88 \\
3.50 & 15.50 & 0.77 & 11.97 & 13.29 & 1.01 & 0.69 & 0.73 \\
3.50 & 18.50 & 0.77 & 14.28 & 15.86 & 1.00 & 0.58 & 0.59 \\
4.50 & 14.50 & 0.77 & 11.24 & 12.56 & 1.02 & 0.79 & 0.86 \\
5.50 & 9.50 & 1.26 & 7.81 & 8.87 & 1.02 & 0.94 & 0.98 \\
5.50 & 18.50 & 1.89 & 14.28 & 15.86 & 1.00 & 0.65 & 0.68 \\
6.50 & 16.50 & 1.89 & 12.86 & 14.41 & 1.01 & 0.76 & 0.81 \\
7.50 & 18.50 & 2.64 & 14.35 & 15.99 & 1.00 & 0.70 & 0.74 \\
9.50 & 16.50 & 3.51 & 13.13 & 14.83 & 1.02 & 0.83 & 0.91 \\
9.90 & 18.50 & 5.62 & 14.56 & 16.37 & 1.00 & 0.75 & 0.80 \\
11.70 & 18.10 & 6.11 & 14.42 & 16.24 & 1.00 & 0.79 & 0.86 \\
14.10 & 19.20 & 8.53 & 15.35 & 17.34 & 1.00 & 0.78 & 0.84 \\
16.40 & 19.50 & 12.40 & 15.70 & 17.79 & 1.00 & 0.80 & 0.85 \\
\hline
\end{tabular}

The points addressed above would constitute a basic description of the probabilistic approach, introducing the reader to the fundamentals of the probabilistic characterization of the ocean waves. However, some questions remain unanswered, such as which is the ideal time duration of the recorded wave signal to estimate the sea spectrum? and how does the time duration of the records affect the repeatability of the same sea spectrum? There are some works, as for example (GODA, 2000) and (MELLO, 2005), that analyze this topic and attempt to provide an appropriate methodology to estimate the ideal sample time. Nevertheless, the procedures proposed have some limitations regarding the bandwidth of the sea spectrum, specially when it comes to bimodal wave spectra. Since this thesis does not aim at providing a systematic analysis of the probabilistic techniques to describe the ocean waves, a discussion regarding these points has been included in 
appendix B, along with a guideline to define the optimal sample time for each event (sea condition).

\subsection{Ship motions in waves}

The main aspects of the linear modelling of floating vessel motions are described in the following. For a detailed derivation and description of the forces and moments acting on the floating body in waves the reader is referred to (NEWMAN, 1977).

First of all, the main assumptions adopted to develop the linear modelling of the ship motions are given by:

1. When defining wave excitation, the same linear model is used as when describing the linear wave theory, i.e. waves are assumed to have a low amplitude and slopes;

2. The motions of the floating vessel (ship/platform) are assumed small;

3. Hydrostatic forces and moments are represented by initial stability approximation (this assumption can be assumed as a consequence of the previous one);

4. Potential flow model is used, or in other words, the flow model is inviscid and irrotational.

5. The boundary layer must be thin enough to guarantee that the outer pressure (estimated by means of the potential flow model) stands as a good approximation for the pressure acting on the body's surface (the hull of the vessel, in our case). In order to guarantee this condition high Reynolds Number are required (in typical marine engineering applications this requirement is fulfilled) as well as a small KeuleganCarpenter number ${ }^{5}$, which ensures that separation effects can be neglected in the most general cases;

6. The quantities used to describe interaction between the hull and water are independent upon the motions.

Regarding the equations of motion of the floating system, they can be derived using the second Newton's law,

\footnotetext{
${ }^{5}$ Section 3.3.1 provides a systematical description of this quantity.
} 


$$
\sum \mathbf{F}=\mathbf{M} \ddot{\xi}
$$

where $\boldsymbol{\xi}=[\delta x, \delta y, \delta z, \delta \phi, \delta \theta, \delta \psi]^{T}$ are amplitudes of the vessel motions and $M$ is mass and inertia moment matrix. Concerning the forces acting on the system, they are given by:

1. The pressure force, $F_{p}$, which can be decomposed as (see (NEWMAN, 1977) and (FALTINSEN, 1993)):

- The so-called Froude-Krylov excitation term: That estimates the force that would be exerted on a fixed body by the incident waves (the vessel does not affect the incoming wave and resulting flow);

- A second term also known as the diffraction part: This component takes into account the wave deformation due to the vessel presence.

It should be emphasized that this decomposition of the resulting forces acting on the floating platform is possible due to the assumption of small wave amplitudes and slope, and small body displacements around a reference point. Moreover, for long waves ${ }^{6}$ the Froude-Krylov forces are dominant meanwhile in short waves both terms must be considered.

2. Another component of the hydrodynamic forces acting on a floating vessel is the radiation term, referred in the following as $F_{h}$. Cummins (1962) showed that the (radiation-induced) hydrodynamic forces in an ideal fluid can be related to a added mass, $a$, and a potential damping, $B$.

3. Finally, the equations of motion are completed by the total static linearized forces, which include the forces due to the body weight, $F_{h s}$.

Thus, Eq. (3.23) can be rewritten as,

$$
\mathbf{F}_{p}+\mathbf{F}_{h}+\mathbf{F}_{h s}=\mathbf{M} \ddot{\xi}
$$

\footnotetext{
${ }^{6}$ The relation between the wave length and the frequency is given by the dispersion relation (GODA, 2000). For deep water applications (such as is the semisubmersible platform under study) the wave length is inversely proportional to the square of the frequency.
} 
Now, it is assumed that the responses of the vessel are linearly related to the amplitude and slope of the regular wave, which velocity potential has been defined in Eq. (3.9). Hence, the response of the $2^{\text {nd }}$ - order system can be fitted to a linear model and the solution is sought in the frequency-domain. Accordingly, Eq. (3.24) can be transformed to the frequency-domain as follows,

$$
[\mathbf{M}+\mathbf{a}(\omega)] \ddot{\boldsymbol{\xi}}+\mathbf{B}(\omega) \dot{\boldsymbol{\xi}}+\mathbf{c} \boldsymbol{\xi}=\boldsymbol{\xi}\left\{-\omega^{2}[\mathbf{M}+\mathbf{a}]+i \omega \mathbf{b}+\mathbf{c}\right\}=A \mathbf{X}
$$

where $\mathbf{M}$ is the system mass and inertia moment matrix, $\mathbf{b}$ represents the linear damping coefficient, $\mathbf{c}$ is the hydrostatic restoring coefficient, $A$ stands as the wave amplitude and $\mathbf{X}$ is the complex amplitude of the exciting force or moment ${ }^{7}$. Through Eq. (3.25) it is possible, by means of standard matrix inversion techniques, to compute the ratio $\boldsymbol{\xi} / A$. Therefore, for the $j^{\text {th }}$ mode, this ratio is given by,

$$
Z_{j}(\omega, \beta)=\frac{\xi_{j}}{A}=\sum_{i=1}^{6} \frac{X_{i}}{-\omega^{2}\left[M_{i j}+a_{i j}\right]+i \omega b_{i j}+c_{i j}}=A_{j}(\omega, \beta)+i B_{j}(\omega, \beta) .
$$

where $Z(\omega, \beta)$ stands as the complex amplitude of the body motion in response to an incident wave of unitary amplitude, frequency $\omega$ and direction of incidence, $\beta$, of the wave in relation to the vessel (see Fig. 3.6). This function is also known as the Response Amplitude Operator (RAO) in the field of marine engineering.

Moreover, it is important to highlight that in Eq. (3.26) the complex notation has been adopted (denoted by $i$ ) aiming to properly compute the phase shift between the responses of the platform and the incident waves. In the last place, $A_{j}(\omega, \beta)$ and $B_{j}(\omega, \beta)$ correspond to real and imaginary parts of the complex transfer function of motion $Z_{j}(\omega, \beta)$.

For instance, the phase angle, $\varphi_{j}$, can be computed as,

$$
\varphi_{j}=\arctan \left(\frac{B_{j}}{A_{j}}\right)
$$

Thus, the complex transfer function is rewritten as,

\footnotetext{
${ }^{7}$ (NEWMAN, 1977) stands as a reference for a comprehensive description and derivation of $\mathbf{X}$.
} 


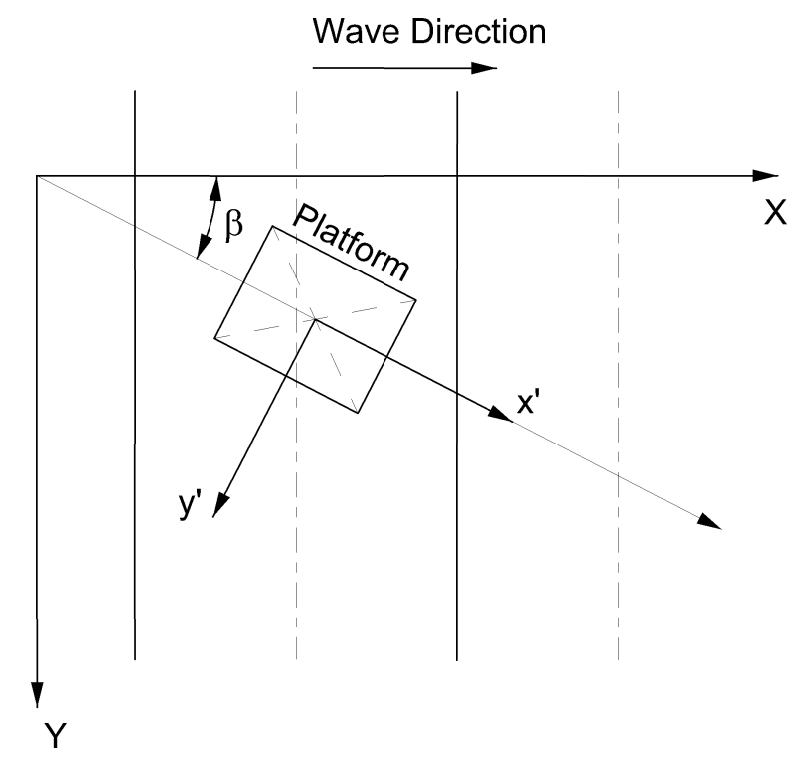

Figure 3.6: Encounter $\beta$ angle: given by the difference between the wave direction and the heading of the floating vessel.

$$
Z_{j}(\omega, \beta)=\left|Z_{j}(\omega, \beta)\right| e^{i \varphi_{j}}
$$

In some cases it is more convenient to define the transfer function in wave period rather than in wave frequency. Then, the equivalence is directly computed as follows,

$$
Z_{j}(\omega, \beta)=Z_{j}\left(T=\frac{2 \pi}{\omega}, \beta\right)
$$

Finally, from Eq. (3.28) it is possible to compute the RAOs for a given direction and frequency range, in virtue of the superposition principle (previously discussed in section 3.2). As for the waves, the responses of the vessel are a random process. Therefore, the response PSD can be adopted to characterize its motions. Formally, the response power spectrum of the $j^{\text {th }}$ mode is related to the RAOs (of the same $j^{\text {th }}$ mode) and the wave spectrum (defined in Eq. (3.19)) by,

$$
S_{j j}(\omega, \beta)=\left|Z_{j}(\omega, \beta)\right|^{2} S_{\eta \eta}(\omega, \beta) .
$$

Among the different qualitative characteristics of the RAOs, it is interesting to highlight its main feature for the intermediate range of frequencies. As in the case of a 
mechanical oscillator, the floating platform will experience a response of large amplitude close and at the natural frequency of each one of its motions (also there is a phase shift $\varphi$ from zero, in lower frequencies, to $-\pi$, for $\left.\omega=\omega_{n}\right)$. The resulting response, thus, will be inversely proportional to the damping coefficient.

Accordingly, the estimation of the RAOs is possible if the potential coefficient $a$ and $B$ are known. However, this estimation will not be accurate unless viscous damping effects are included. This is due to the fact that the model adopted until now assumes that the fluid is inviscid. Therefore, in those cases where the damping is dominated by viscous effects the theory will result in misleading (over)-estimation of the responses that the floating system presents close to its motion resonances. A brief discussion regarding the viscous damping and its relevance for the motions of floating structures can be followed in the next section.

\subsubsection{Viscous damping effects}

Returning to the analogy of the mechanical oscillator, it is widely known that the resonant response will be inversely proportional to the damping coefficient. Hence, offshore structures with small damping coefficients will experience large resonant responses. As a matter of fact, it can be shown (by means of the Haskind relations ${ }^{8}$ ) that there is a connection between the exciting force and the potential damping coefficient. Consequently, the vessels designed to experience small exciting forces will be subjected to small damping coefficients, leading to large resonant responses. Clauss et al. (2014) show that the semisubmersible platforms fall within this category and that their motions will be characterized by large resonant responses, whose damping is dominated by viscous effects mainly coming from the separation of the flow over the columns and pontoons.

Following the approach proposed by Morison, Johnson and Schaaf (1950), the nonlinear drag force originated by viscous effects and the associated downstream wake can be expressed as,

$$
\mathrm{d} F_{d}=\frac{1}{2} C_{d} \rho|u| u \mathrm{~d} S
$$

\footnotetext{
${ }^{8}$ See (NEWMAN, 1977).
} 
where $u$ is the total local flow velocity, the $C_{d}$ stands as the drag coefficient (including the form and friction drag) and $S$ is the cross sectional area of the element of the structure under study.

Now, using Eqs. (3.9) and (3.31), it can be shown that the nonlinear drag force fulfils the following relation,

$$
\mathrm{d} F_{d} \propto|\cos (\omega t)| \cos (\omega t)
$$

And using series to develop the term $|\cos (\omega t)| \cos (\omega t)$, the drag force can be rewritten as,

$$
\begin{aligned}
\mathrm{d} F_{d} & =\sum_{n=0}^{\infty} \frac{(-1)^{n} 8 \cos (2 n+1) \omega t}{\pi(2 n+1)\left[4-(2 n+1)^{2}\right]} \frac{1}{2} C_{d} \rho u^{2} \mathrm{~d} S \\
& \approx\left[\frac{8}{3 \pi} \cos (\omega t)+\frac{8}{15 \pi} \cos (3 \omega t)\right] \frac{1}{2} C_{d} \rho u^{2} \mathrm{~d} S
\end{aligned}
$$

This non-linear force can be linearized by means of the principle of energy equivalence: Where the energy dissipation resulting from the drag force must be identical in both linear and nonlinear terms. More details regarding this linearization are provided in section 5.2.4.

As a direct consequence of this linearization, the estimated drag coefficient can be superposed to the radiation damping coefficient for a proper computation of the RAO (close to the motion resonance, where an over-estimation of the responses may occur).

Concerning the resulting linearized damping coefficient (see Eq. (3.33)), it includes both frictional and separation effects. However, it has been identified that the viscous damping for offshore structures is mainly governed by the separation effects (SARPKAYA, 2010). A common approach to categorize the relevance of the separation effects in periodic flows is the computation of the Keulegan-Carpenter (KC) number. This coefficient is formally expressed as,

$$
K C=\frac{U T}{D},
$$

where $U$ is the equivalent amplitude of the object (or flow) velocity oscillation, $T$ rep- 
resents the period of the oscillation and $D$ stands as a characteristic length scale of the object (e.g. the diameter for a vertical cylinder under wave loading). Moreover, following the linear wave theory for deep water conditions (see (NEWMAN, 1977)) $U$ can be rewritten as $U=A 2 \pi \exp \{k y\}$. Therefore, Eq. (3.34) can be expressed as,

$$
K C=\frac{h \pi}{D} \exp \{k y\},
$$

where $h=2 A$ stands as the wave height.

Physically, the $\mathrm{KC}$ number can be related to the ratio of the maximum values of the drag and inertia force acting on an element of the structure (see (CLAUSS et al., 2014)) and it is crucial for determining the nature of the fluid force acting on the structure. Therefore, when the $\mathrm{KC}$ number is small the inertia forces are more important than the drag forces, whereas if the $\mathrm{KC}$ number is large the drag forces are dominant. In section 3.3.2 the $\mathrm{KC}$ number has been used to classify the nature of the forces acting on the column elements of the hull of the semisubmersible platform adopted as a case study in this thesis.

Finally, it must be highlighted that in this thesis the solution of the linear equations that describe the motions of the vessel in waves has been computed by means of numerical methods, which allow the estimations of the RAOs for arbitrary geometries. More precisely, the RAOs adopted to estimate the sea conditions were computed by means of two different software packages. First, WAMIT (LEE; NEWMAN, 2006) software was used to analyze the wave-body interaction in the frequency-domain. Secondly, ANSYS-AQWA (ANSYS, 2013) has also been used in this thesis. This package includes both frequency and time-domain analysis.

\subsubsection{Note on the motions of semisubmersible platforms in waves}

The overview provided above constitutes a general description of the most significant definitions and theory adopted to characterize the induced ship motions in waves. Since this thesis aims to assess the use of a semisubmersible platform as a motion based wave sensor, a discussion regarding the main characteristics of its hydrodynamic properties is provided herein. 
At the beginning of section 3.3 it has been shown that the interaction of waves with floating structures includes the effects that the vessel presence has on the incoming flow. The significance of this effect is associated to the shape, dimensions and relative motion of the vessel that is being analyzed. In order to categorize the interaction wave-body, distinction must be made between small and large bodies, since the nature of the forces experienced by the vessel differs significatively depending on its size. In this case, the reader is referred once again to (CLAUSS et al., 2014) for a detailed discussion about this topic.

The categorization of small/large bodies is based on the comparison between the characteristic length of the structure, $D$, the characteristic wave length, $\lambda$, and the wave height, $H$ (these parameters are generally evaluated for the most adverse conditions, i.e. at $y=0)$. Therefore, for small $D / \lambda$ and large values of $H / D$ the structure can be assumed small. These conditions lead to a regime where viscous drag forces dominate (mainly originated by flow separation). For wave-body interactions where $D / \lambda$ are larger than 0.2 and $H / D$ is small, diffraction effects will dominate the dynamics of the structure and separation effects can be neglected.

In order to illustrate this categorization Fig. 3.7 is provided below, where the loading regimes for the columns of the Åsgard-B semisubmersible platform have been assessed in terms of the columns diameters, significant wave height and peak period for all the sea conditions tested during the experimental campaign ${ }^{9}$. In this figure the vertical axis shows the ratio $H / D$, which is equivalent to the KC number (CLAUSS et al., 2014) while the horizontal axis can be interpreted as a the diffraction parameter.

The results in Fig. 3.7 show that the diffraction effects are more dominant for the columns with large diameters than for the columns with a diameter equal to $12.2 \mathrm{~m}$, as expected. Moreover, for extreme wave conditions (high $H / D$ relations) the data shows that the drag effects become relevant, being the columns with smallest diameter the ones that will experience larger drag forces. Meanwhile, in calm-mild sea conditions these hull elements will experience loading regimes featured by a dominance of the inertia terms.

This discussion regarding the main characteristics of the wave-body interaction pro-

\footnotetext{
${ }^{9}$ Chapter 5 includes detailed descriptions of the main dimensions of the platform as well as the sea conditions tested.
} 


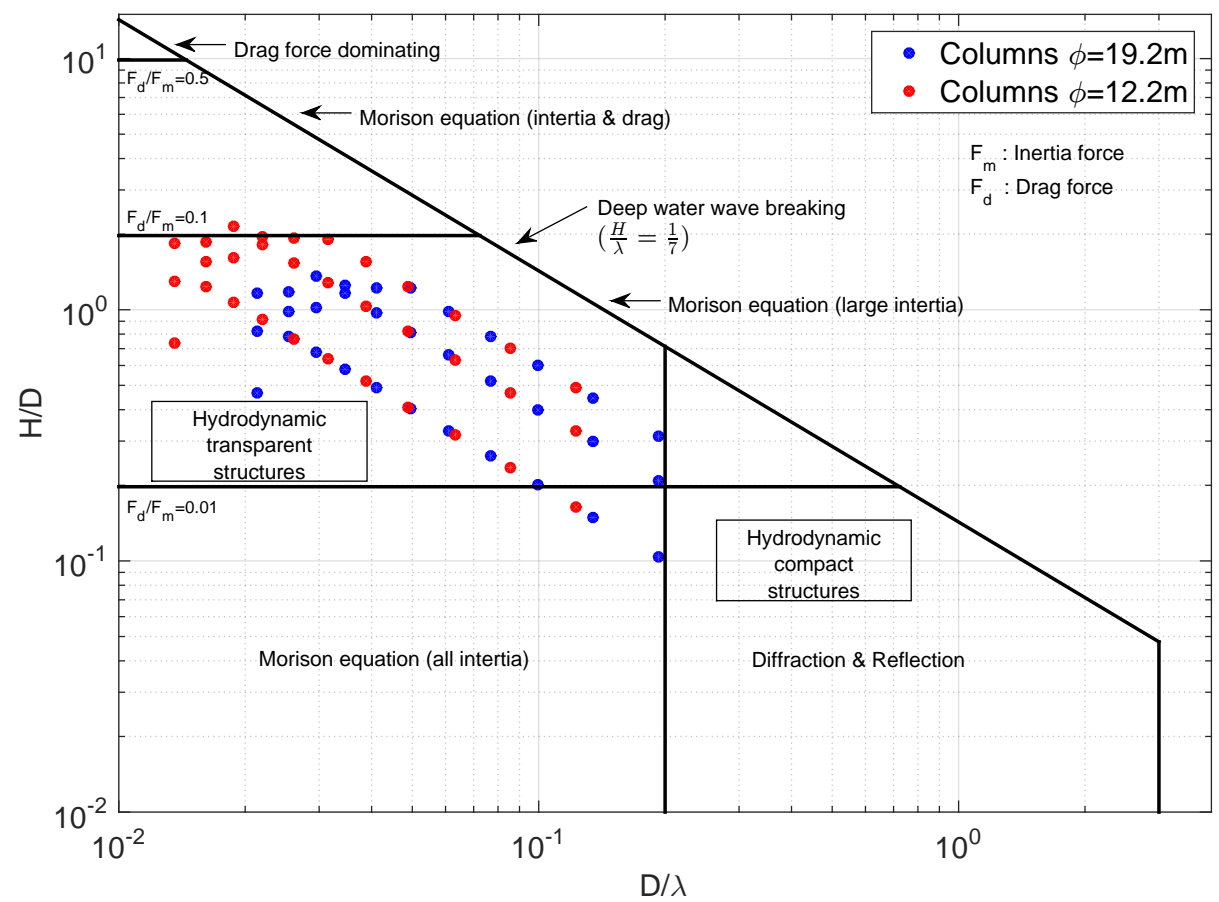

Figure 3.7: Loading regimes of horizontal forces acting on the columns of the Åsgard-B semisubmersible platform in deepwater. Inspired from (CHAKRABARTI, 2005).

vide a general idea of the dominant effects that column elements of the hull will experience. Notwithstanding this, the main characteristics of the platform dynamics are given by the motion RAOs. Thus, a brief review of the main feature of the motion RAOs for a semisubmersible platform is provided hereafter.

First, it is analyzed what happens to all the six degrees of motion in the high frequency range. At very high frequencies, the exciting force in the numerator of Eq. (3.26) will tend to zero for all modes of motion (NEWMAN, 1977) while the denominator would be proportional to $\omega^{2}$. Thus, for short waves the RAOs of the six dofs will tend to zero.

In the long waves regime (that correspond to the limit of low frequencies), differently from the high frequency range, the platform motions are not expected to present the same behavior. Regarding the roll and pitch motions, it can be show that the exciting force will be characterized by small values, see (NEWMAN, 1977), while the denominator of Eq.(3.26) will be given by the respective restoring coefficients (which are different of zero). For the heave motion, it is expected that the structure will simply ride up and down along with the free surface. In relation to the horizontal modes of the platform, the large diameter columns and massive displacements that the semisubmersible platforms 
present will result in a greater capability of the hull to disrupt the incoming waves. As a consequence, the resulting forces may induce large amplitudes of motions of the platform in these dofs.

For the intermediate frequency range, it is already known that the main features of the RAOs are the resonant responses of the motions. The resonant frequencies of the heave, roll and pitch motions are computed using the geometric features of the platform (CLAUSS et al., 2014). For instance, the distribution of the waterplane area of a semisubmersible platform results in large resonant periods for these motions, when compared with a ship-shaped platforms characterized with similar displacements. As a matter of fact, if compared with ship-shaped platforms, such as FPSOs, semisubmersible platforms are characterized by smaller waterplane areas, which result in low hydrostatic restoring coefficients. Furthermore, layout of the waterplane area of a semisubmersible platform (given by the cross sectional area of the platform's columns) leads to large moments of inertia. Altogether, these characteristics result in large values for the resonance periods of heave, roll and pitch motions. For the sake of illustration, the natural periods for the heave, roll and pitch motion of the Åsgard-B semisubmersible platform are: $24 \mathrm{~s}, 74 \mathrm{~s}$ and $88 \mathrm{~s}$, respectively ${ }^{10}$. That are significantly larger than the ones that characterize the dynamics of a FPSO, given for example in (BISPO et al., 2016), and that corresponds to $11.5 \mathrm{~s}$, $14.5 \mathrm{~s}$ and $16.3 \mathrm{~s}$, for the heave, roll and pitch motions respectively.

For illustrative purposes, an example of the heave motion RAO of the semisubmersible platform used during the experimental campaign is shown in Fig. 3.8. This RAO has been computed in the frequency-domain using WAMIT for a heading condition equal to $180^{\circ}$, including the phase $\varphi$. From this figure, one may realize that this motion presents its resonance close to $24 \mathrm{~s}$. Also, in 22s the RAO has a cancellation point. For practical reasons, it is worth remembering that this cancellation point is the result of the equilibrium between the forces acting on the pontoons of the platform and the forces acting on the columns ${ }^{11}$. Analogous behavior is observed at lower wave periods, or higher frequencies, where the heave RAO shows some zero points. Nonetheless, in this case the origin of the zero points is due to the cancellation of the wave effects by the superposition of the forces on both sides of the semisubmersible. It is important to highlight that for some sea conditions the

\footnotetext{
${ }^{10}$ The main characteristics of these motions are thoroughly discussed in chapter 5 .

${ }^{11}$ See appendix D for a comprehensive analysis of the heave RAO of the semisubmersible platform.
} 
platforms may present responses in frequencies that correspond to cancellation points of the RAOs, where the platform is not expected to present any significant responses. This deviation from the theoretical transfer function can be related, among other factors, to non-linear drag effects; once again the reader is referred to appendix D for a detailed discussion regarding the cancellation points of the heave RAO. Finally, the RAO shows that for long waves the heave motion responds by following the surface elevation, meanwhile for short waves the platform is expected to present motions with small amplitudes, as expected.

Concerning the phase of the $\mathrm{RAO}, \varphi$, negative values mean that the positive maximum of forces or structural motions occurs after the passage of the wave crest. For instance, if $\varphi=-\pi$ we have that platform vertically oscillates opposite to the wave elevation. The positives values for the phase stand for conditions where the positive maximum of forces happens before the passage of the wave crest. In long waves, the semisubmersible predictably follows the wave amplitude, and the resulting phase angle between heave motion and wave elevation is zero.

With regard to the horizontal modes of the platform, their resonances would depend on the restoring coefficients induced by the mooring system. Since the submerged lines result in a system with low stiffness while the whole system is characterized by a large mass, the resulting resonances of these motions will happen in the very low frequency range. It is important to highlight the fact that VMB wave inference aims at estimating gravity waves, whose periods usually range between 1 and 30s. Thus, the effects that may arise close to the resonances of these motions $(>>30 \mathrm{~s})$ are not of interest for the purpose of this work.

The importance of the resonance frequency has its origin in the resulting amplitudes of motion. It has been shown that close and at the resonant frequency, the system will present responses of large amplitudes. Furthermore, the semisubmersible platform is characterized by large resonance responses, in virtue of the connection between the exciting force and the damping coefficient as explained previously in section 3.3.1. As a matter of fact, radiated waves are expected to be small due large draft of the platform and the cylindrical shape of the columns. Therefore, the platform will experience small exciting forces and, as a consequence, it will be subjected to small damping coefficients. 

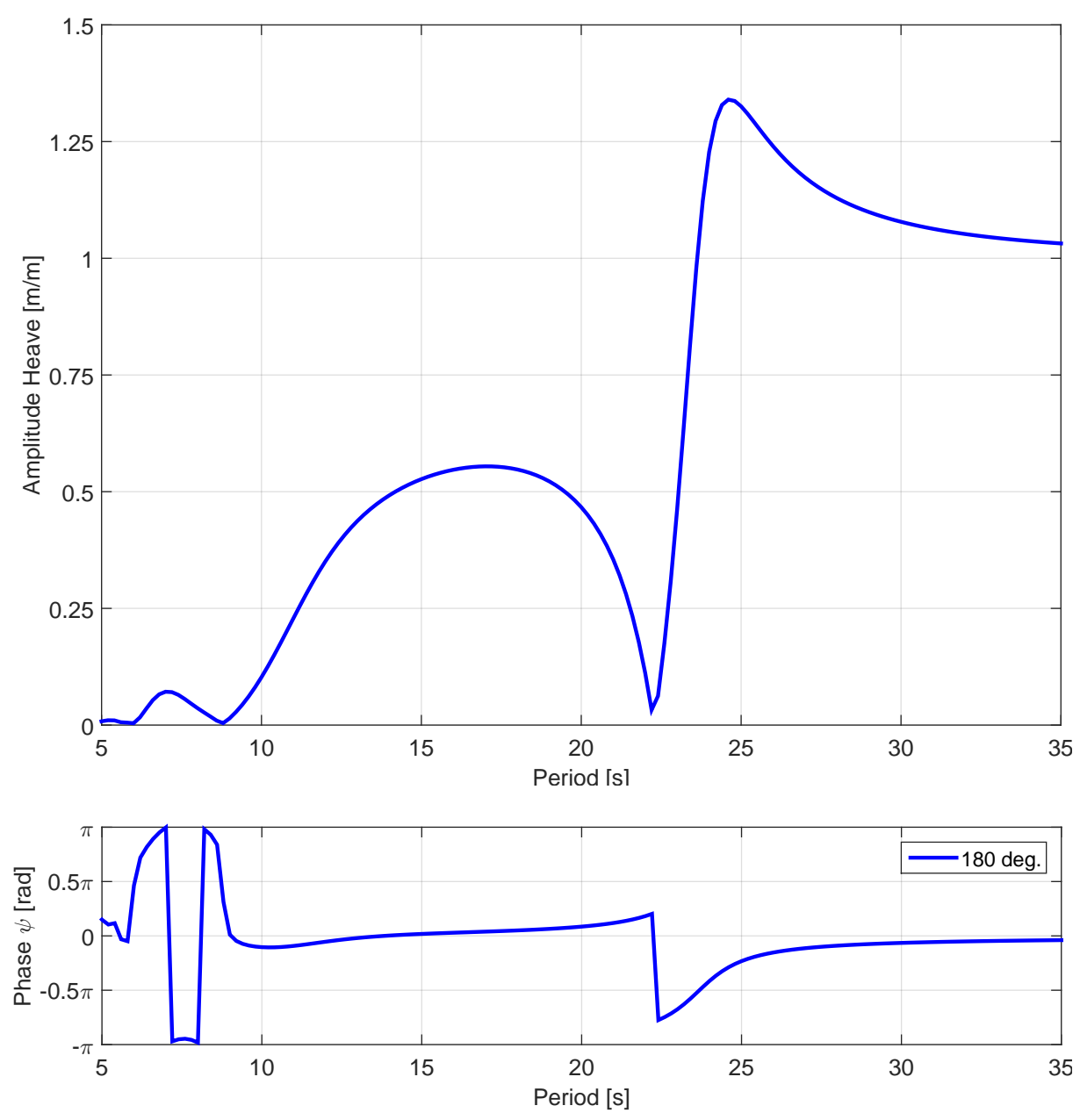

Figure 3.8: Heave motion RAO.

In order to illustrate the relevance of these viscous drag effects on the heave motion response, Fig. 3.9 provides the Keulegan-Carpenter (KC) number estimated for the pontoons of the platform, based on the measured responses close to the resonance of the motion. These values were computed using a significant amplitude of the heave motion derived from Eq. (3.15), which is given by,

$$
A_{z}=\sqrt{2 S_{z z}\left(\omega_{n}\right) \cdot \delta \omega_{n}}
$$

where $S_{z z}\left(\omega_{n}\right)$ is the measured power spectrum of the heave motion at the natural frequency and $\delta \omega_{n}$ is the frequency interval of the spectral analysis close to the natural frequency $\left(\omega_{n}\right)$, or $\left[\omega_{n}-\omega_{(n-1)}, \omega_{(n+1)}+\omega_{n}\right]$.

In this case the expected effect of the wave flow on the damping force is small due to 


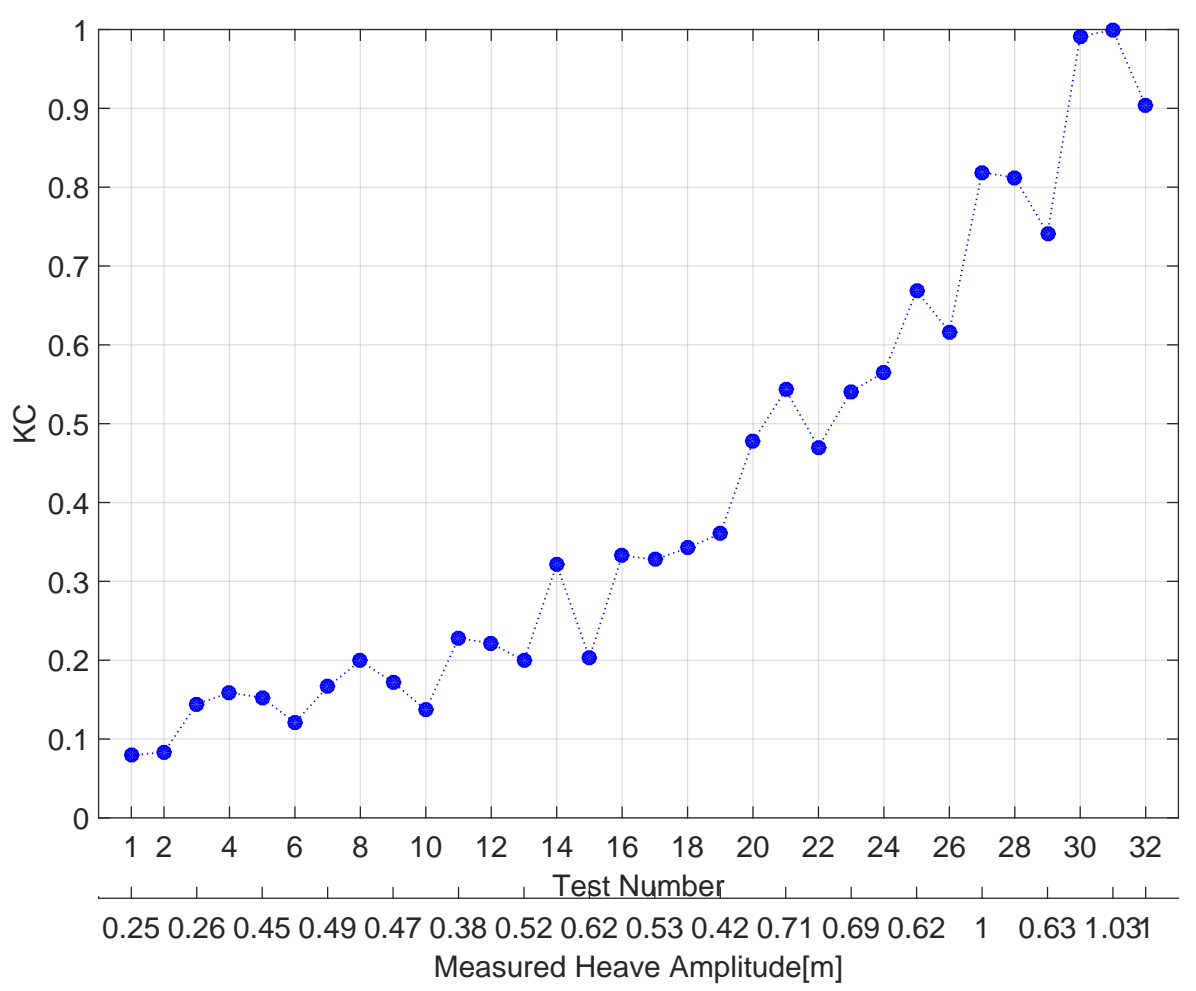

Figure 3.9: Keulegan-Carpenter values estimated for the pontoons of the semisubmersible platform near resonance.

the large draft of the platform. Accordingly, the KC number has been defined as,

$$
K C=\frac{2 \pi A_{z}}{h_{\text {pont }}} .
$$

being $A$ the significant amplitude of the heave motion (see Eq. (3.27)) and $h_{\text {pont }}$ the height of the pontoon.

Therefore, when the KC number is small the inertia forces are more important than the drag forces, whereas if the $\mathrm{KC}$ number is large the drag forces are dominant. Particularly, the results in Fig. 3.9 attest that sea conditions with high periods will increase the effects of the drag forces on the pontoon near the resonance of the motion. Similar results are presented by Chaplin (1988), who analyzed the flow around a submerged circular cylinder in beam waves. Moreover, the author shown that since the drag forces arise mainly from separations effects, there are not any severe scale effects (a detailed discussion regarding the scale effects is also provided in (FALTINSEN, 1993)), thus increasing the reliability of the model-scale results. 


\subsection{The semisubmersible platform as a VMB wave sensor}

This section aims at providing an earlier assessment of the possible advantages and disadvantages that the adoption of the semisubmersible platform as a VMB wave sensor may present. This evaluation is entirely based on the main weaknesses and strengths related to the methodology described in section 3.3. Although the following discussion is focused on the dynamics of the semisubmersible platform, the reader is referred to chapter 4 and appendix B for a discussion concerning the main limitations of the theory provided in section 3.2 and its possible implications for the VMB wave inference problem.

The main objective of this thesis is the assessment of the use of the semisubmersible platform as a motion based wave sensor. Nonetheless, other ship-shaped vessels, such as FPSOs and container ships, have been adopted as reliable wave sensors in a wide range of sea conditions. Therefore, for illustrative purposes the main features of the semisubmersible platform have been compared with the ones of the FPSOs, which are broadly documented in the literature, e.g. (BISPO, 2011b).

The RAOs of the Åsgard-B semisubmersible platform are provided in Fig. 3.10. This figure includes the RAOs corresponding to: surge, sway, heave, roll and pitch motions ${ }^{12}$, which have been computed for 5 different headings. In a similar way, and only for comparative purposes, Fig. 3.10 provides the RAOs of a FPSO.

Concerning the RAOs of the semisubmersible platform, one may realize that in the first region up to the zero point at $T=10 \mathrm{~s}$ in the horizontal motions, the transfer functions of motion indicate small oscillation amplitudes. As a consequence, unlike the ship-shaped platforms (see Fig. 3.11), the semisubmersible is expected to filter large amounts of energy for the most frequently encountered wave periods in offshore activities $(T<10 \mathrm{~s})$. For instance, the heave motion will be less than $10 \%$ of wave elevation. These characteristics of the semisubmersible motions in the high frequency range properly illustrate the main possible drawback of the VMB wave inference, highlighted previously in section 1.1.2, which may result in inaccurate estimations of calm sea conditions.

\footnotetext{
${ }^{12}$ The yaw motion has not been included in this analysis due to the fact that its main properties depend on external factors, such as mooring stiffness and inertia properties of the platform around the $z$ (vertical)-axis; about which there was not accurate/enough data in order to carry out a proper analysis and characterization.
} 

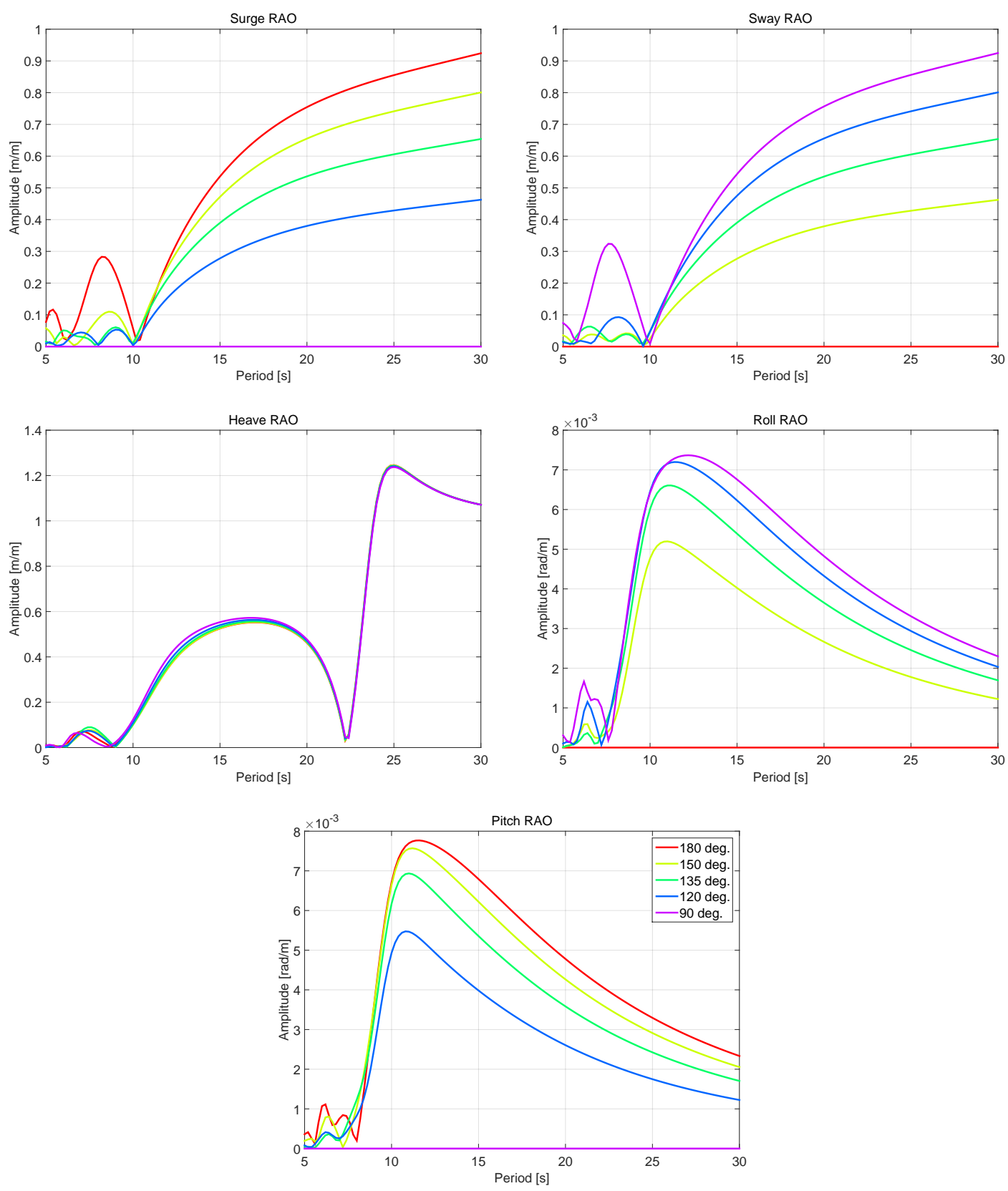

Figure 3.10: RAOs of the Åsgard-B semisubmersible platform. From left-to-right then top-to-bottom: surge, sway, heave, roll and pitch.

In the intermediate range $(10 \mathrm{~s}<T<20 \mathrm{~s})$ the RAOs of the semisubmersible platform still show smaller oscillation amplitudes than the ones presented by the FPSO. For instance, the small amplitudes that the semisubmersible platforms present in calm to mild sea conditions have led some authors to assume (a priori) that these platforms are not reliable motion based wave sensors, as it has been discussed in section 1.1.2. However, one may realize that although the RAOs are not characterized by large responses, there are no resonance or cancellation points in this interval in any of the five dofs under study. 

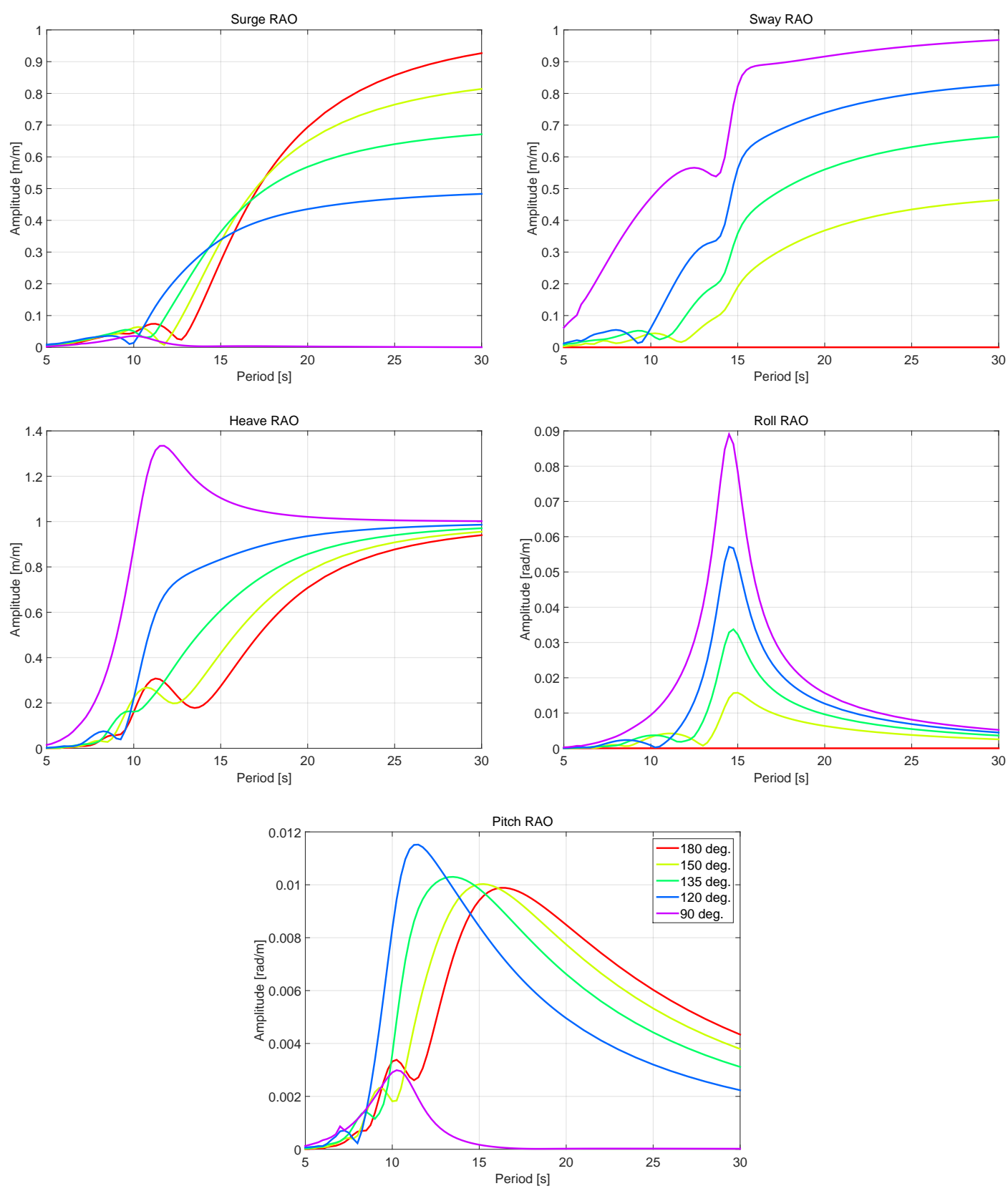

Figure 3.11: RAOs of a FPSO. From left-to-right then top-to-bottom: surge, sway, heave, roll and pitch. Data from (BISPO, 2011b).

Therefore, the reliability of the linear relation between the exciting waves and the platform responses is hold. Concerning the RAOs of the FPSO, they not only show several zero points but also the heave, roll and pitch motions present their resonances within the limits of this period range. Thus, the associated non-linear effects are expected to result in the increase of the uncertainty of the estimations of the sea conditions based on the measured motions of a FPSO (BISPO, 2011b). 
For the highest possible waves to which the extreme wave conditions belongs, periods lie in the region of $T>20 \mathrm{~s}$. The RAOs have been computed following the approach described in section 3.3, where the small wave amplitudes/slopes are assumed. However, it is not clear if this assumption is consistent for the period range evaluated: Although for the most general sea conditions the wave amplitude/slope can be assumed sufficiently small (see Fig. 3.7), in extreme sea conditions with very step wind waves this assumption it may not be fulfilled leading to uncertainties of the RAOs. The most significant features of the semisubmersible RAOs in this range are identified for the heave motion, that presents a cancellation point and its resonance.

Regarding the dynamic behavior of the heave resonance, it is already known that the semisubmersible platforms are expected to present large responses, due to the small potential damping that the hull presents. This may result in the violation of the assumption of small response amplitudes. Moreover, non-linear viscous forces become significant in the heave motion near the resonance, modifying the linearly estimated responses as well as the cancellation point close to the resonance (here the reader is referred, once again, to appendix $\mathrm{D}$ for a detailed discussion regarding the resonance and this zero point). In this case, an external damping coefficient must be imposed in order to emulate the effects of the viscous forces. However, it is not obvious how to bound the influence of these viscous effects a priori (a detailed evaluation of the cancellation point and the assessment of various solutions proposed to account for the associated shortcomings is provided in chapter 5). Therefore, in harsh weather conditions the heave responses must be properly modelled in order to avoid misleading estimation of the sea conditions that induced the measured motions of the platform. Regarding the extremely low-frequency components, it can be highlighted that the platform is expected to follow the free surface elevation, reaching, in the limit, a pure heave motion.

Finally, another characteristic of the semisubmersible platforms is their reduced value of the metacentric height $(\mathrm{GM})^{13}$, which limits its stability properties. As a consequence, these platforms constantly monitor their GM values. Moreover, usually these platforms are typically designed only for three different draft configurations which are: the operational, survival and transit draft. In the operating condition the draft is at the maximum

\footnotetext{
${ }^{13}$ The metacentric height stands as a measurement of the initial static stability of a floating body. A detailed analysis of this parameter is found in (LEWIS, 1988)
} 
magnitude. These features turn to be an advantage for the semisubmersible platforms as motion based wave sensor, since they allow the estimation of the most appropriate set of RAOs at any moment. Contrary, the FPSOs present large draft variations that result in significant changes of their stability properties, such as their GM and centre of gravity. This limitation is well illustrated in (BISPO, 2011b), where the author carried out a sensibility study to assess the RAOs changes of a FPSO in differing loading conditions. 


\section{BAYESIAN INFERENCE}

\subsection{The Bayesian paradigm}

Previous works and results concerning VMB wave inference, e.g. (ISEKI; OHTSU, 2000) and (NIELSEN, 2006), as well as the preceding research conducted at the USP on this topic, i.e. (TANNURI et al., 2003; SIMOS et al., 2012), have motivated the adoption of the Bayesian inference method to estimate the sea conditions based on the measured platform motions. A proper explanation with regards to the advantages of using this statistical inference method to estimate the sea conditions from the measured platform motions is addressed along the next sections.

Bayesian inference is one of the most important statistical inference methods in use nowadays. Its core principle is that the posterior distribution of the variable of interest is the result of updating the existing prior information on the model parameters using the available data, where the systematic process of learning from the data is implemented via Bayes theorem. This can be formally expressed as follows (BERNARDO; SMITH, 2006),

$$
P(x \mid B)=\frac{P(B \mid x) p(x)}{P(B)},
$$

where $P(x \mid B)$ is the posterior updated distribution, $P(B \mid x)$ is the probability of observing $B$ given $x, p(x)$ is the prior distribution and $P(B)$ stands for the marginal probability distribution of observing the event $B$.

Since the proportionality constant, $P(B)=\int_{\omega} P(B \mid x) p(x) \mathrm{d} x$, may always be deducted from the fact that $P(x \mid B)$, a probability density function, may be integrated to one, the Bayes theorem may be simply expressed as follows, 


$$
P(x \mid B) \propto P(B \mid x) p(x)
$$

\subsection{Bayesian modelling procedure and ill-conditioned problems}

From a formal Bayesian point of view, it is assumed that the statistical model derived from the observed data, $P(B \mid x)$, is correct. In fact the Bayesian learning process is optimal when $P(B \mid x)$ matches the exact statistical distribution of the observed data (ZELLNER, 1988). Returning to the VMB wave inference problem, the model can be written in accordance with the RAOs of the vessel. Thus, the output of the Bayesian inference procedure will be optimum for those cases where the RAOs stand as the true (exact) statistic that determine the likely behavior of the vessel when operated at sea. However, in practice, it is already known that only in controlled conditions and for a limited range of frequencies and directions the RAOs will lead to the exact statistical model. For instance, in several engineering problems both the exact statistical model parameters and their number (order) are generally unknown, and oftentimes the exact model is infinite-dimensional (then, non-parametric). Therefore, to attain something close to a exact model, one would need a very large statistical model, yet this is at the expense of keeping the statistical model as simple as possible in order to focus on the main characteristics of the system under study.

One of the first works dealing with this challenge was the problem addressed by Whittaker (1922) in the estimation of a smooth trend. In that problem, the observations, B, are given. They are assumed to consist of the sum of a smooth function, $f$, and the observation Gaussian noise with zero mean and variance $\sigma^{2}$. The problem is to estimate the unknown trend function, $f$. In this context, Whittaker (1922) suggested that the optimal solution will balance a tradeoff between the fitting of data and the goodness-of-fit to a smoothness criterion. This idea is considered a conceptual predecessor of smoothness priors in Bayesian inference.

Consequently, a common approach is to model the phenomenon under investigation making use of a stochastic linear regression model, which allows the study of the essential features of the unknown physical process. 
Consider the Gaussian stochastic linear regression model,

$$
\mathbf{B}=\mathbf{A} \mathbf{x}+\varepsilon
$$

where $\mathbf{B}$ stands for the measurements, $A$ is the matrix that contains the characteristics of the sensor, $\mathbf{x}$ is the unknown vector and $\varepsilon$ is the sensor noise with zero mean and variance $\sigma^{2}$. The dimensions of the matrices in Eq. (4.3) are $\mathbf{B}: n \times 1 ; \mathbf{A}: n \times p ; \mathbf{x}: p \times 1$ and $\varepsilon: n \times 1$, where $n$ is the number of measurements and $p$ is the dimension of the unknown vector $\mathbf{x}$.

A straightforward approach to reconstruct the unknown would be the use of the inverse transform with the inverse filter, which is possible by using the pseudoinverse $\left(\mathbf{A}^{+}\right)$of matrix A. Thus,

$$
\hat{\mathbf{x}}=\mathbf{A}^{+} \mathbf{B}=\mathbf{x}+A^{+} \varepsilon
$$

However, the noise may make this approximation impossible. As a mater of fact, in solving the inverse-problem, the pseudoinverse $\mathbf{A}^{+}$operates as a high-pass filter that has the undesirable tendency to amplify noise. This means that the estimation of the unknown quickly becomes unstable as many noisy values are multiplied by larger values and then summed up. In those cases, the pseudoinverse is said to be ill-conditioned. Otherwise, the problem is known to be well-conditioned or well-posed ${ }^{1}$, leading to a significant variation of the output value of the problem for a small change in the input argument.

The standard approach to solve the problem introduced by Eq. (4.3) is the use of the linear least square regression method finding the minimum of $J(\mathbf{x})=\|\mathbf{A x}-\mathbf{B}\|^{2}$. However, if the problem is ill-conditioned, the ordinary least square estimation leads to an under-determined or, more often, to an over-determined (over-fitted) model ${ }^{2}$. In order

\footnotetext{
${ }^{1}$ In accordance with Hadamard (2014), a well-conditioned problem should have the following properties: (i) a solution exists; (ii) the solution is unique; (iii) the solution's behavior changes continuously with the initial conditions. A simple illustrative example of an ill-conditioned matrix is given by the following matrix,

$$
\mathbf{A}=\frac{1}{2}\left[\begin{array}{cc}
1 & 1 \\
1+10^{-10} & 1-10^{-10}
\end{array}\right] \text {, with } \mathbf{A}^{+}=\left[\begin{array}{cc}
1-10^{10} & 10^{10} \\
1+10^{10} & -10^{10}
\end{array}\right]
$$

Which will result in the amplification of the measurement noise (when it exists). It is important to highlight that this matrix is characterized by a very small determinant $\left(2 \cdot 10^{-10}\right)$.

${ }^{2}$ The reader must note that the nature of the problem is also conditioned by the number of unknowns
} 
to give preference to a particular solution with desirable properties, a regularization term can be included in this minimization,

$$
J(\mathbf{x})=\|\mathbf{A x}-\mathbf{B}\|^{2}+\lambda\|\mathbf{D} \mathbf{x}\|^{2}
$$

where $\lambda$ is referred to as a hyperparameter (LINDLEY; SMITH, 1972), and stands as a trade-off parameter, balancing the variability of the estimation of the unknown and the regularization condition. A small hyperparameter will prioritize a rough estimation and, as a consequence, the data totally determine the estimation. As the hyperparameter gets larger, the estimate moves further from the rough estimation and the regularization conditions become more influential. Concerning matrix $\mathbf{D}$, it stands as the matrix containing the different weights given to regularization and it is usually known. This equation is the more general form of the regularized objective function also known as the weighted regularization (KITAGAWA; GERSCH, 2012).

Accordingly, if $\lambda$ were known and since the measurement noise is assumed Gaussian, the unbiased solution of the problem is given by the minimization of the regularized objective function, Eq. (4.5). Thus, taking the gradient with respect to $\mathbf{x}$,

$$
\nabla J(\mathbf{x})=2 \mathbf{A}^{T}(\mathbf{A} \mathbf{x}-\mathbf{B})+2 \lambda \mathbf{D}^{T} \mathbf{D} \mathbf{x}
$$

and setting this gradient to zero, the unbiased solution is given by,

$$
\hat{\mathbf{x}}=\left(\mathbf{A}^{T} \mathbf{A}+\lambda \mathbf{D}^{T} \mathbf{D}\right)^{-1} \mathbf{A}^{T} \mathbf{B}
$$

Aiming at illustrate the weighted regularization, Fig. 4.1 has been included as an example. This figure provides the exact function (black line) that must be fitted and it is given by $y(x)=1.5 x$. Moreover, 17 points (black points), sampled from the exact function $(y)$, are included. In order to obtain these 17 noisy samples accordingly with Eq. (4.3), the following relation has been adopted: $y+\varepsilon$, where $\varepsilon$ is a Gaussian measurement noise with zero mean and variance equal to 1 . In addition to these samples and the real function $(y)$, approximations obtained by means of a polynomial model of order four are displayed.

that have to be solve. Thus, if $n<p$ (in addition to the fact that the pseudoinverse of matrix $A$ may act as a high pass filter) then the adoption of ordinary multivariate procedures to estimate the unknowns is not longer possible. 


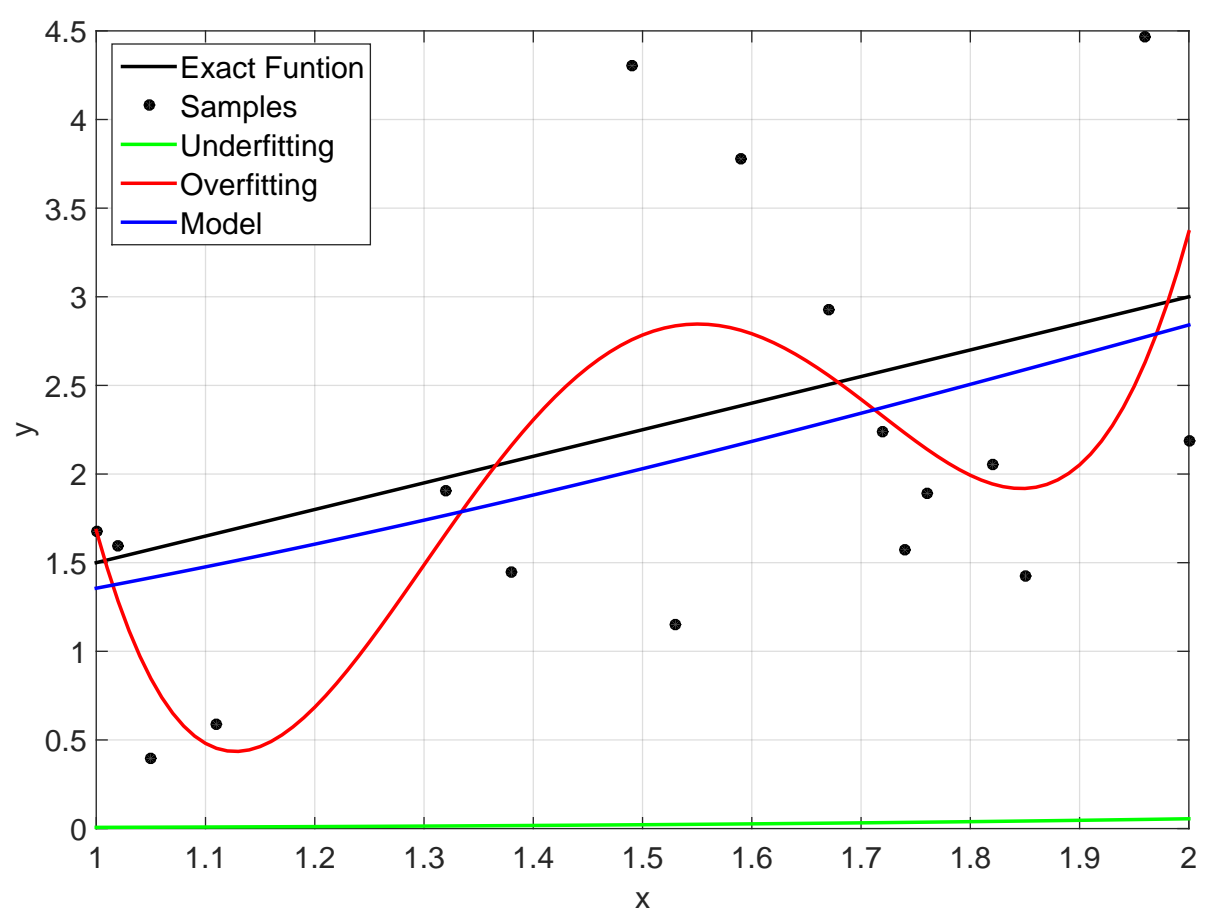

Figure 4.1: Illustration of the weighted regularization problem.

In this case, and with illustrative purposes, a weighted regularization has been adopted to compute the fourth order fitting model. Therefore, the coefficients of the fourth order polynomial models are computed by means of Eq. (4.7). Concerning the matrix containing the different weights, $\mathbf{D}$, the following form has been adopted,

$$
\mathbf{D}=\left[\begin{array}{ccccc}
1 & -2 & 1 & 0 & 0 \\
0 & 1 & -2 & 1 & 0 \\
0 & 0 & 1 & -2 & 1 \\
0 & 0 & 0 & 0.1 & 0.1 \\
1 & 0 & 0 & 0 & 0.1
\end{array}\right]
$$

This matrix results in a regularization problem that prioritizes smooth solutions (the reader is referred to section 4.4 and (KITAGAWA; GERSCH, 2012) for a detailed discussion concerning the main characteristics of matrix $\mathbf{D}$ provided). The hyperparameter, $\lambda$, assessed for this illustrating example varies between 0 and 1000 (this stands as a set of values that, similarly to matrix $\mathbf{D}$, has been selected $a d-h o c)$.

The solutions obtained in this example highlighted the critical role of the hyperpa- 
rameter. First, an estimation of the training data has been obtained using $\lambda=1000$ and it is not sufficient to fit the training samples. This is called underfitting (green line). Secondly, the value of $\lambda$ has been set equal to 0 , which results in a polynomial model (red line) that over-fits the sampled points, i.e. it learns the noise of the training data. Finally, the best fitting model is included (blue line) and it corresponds to $\lambda=30$. This model minimizes the norm of the differences between the coefficients of the fourth degree polynomial model obtained through the exact function, $y=1.5 x$, and the fourth degree polynomial model, $y_{\lambda}$, computed using the weighted regularization with $\lambda=30$. Table 4.1 shows the error values for the three values of $\lambda$ aforementioned.

Table 4.1: Hyperparameter values and the corresponding estimated error.

\begin{tabular}{cc}
\hline$\lambda$ & $\left\|\mathbf{x}_{\lambda}-\mathbf{x}\right\|^{2}$ \\
\hline 0 & 581.3 \\
30 & 0.89 \\
1000 & 1.86 \\
\hline
\end{tabular}

As a matter of fact, $\lambda=30$ is a good choice for the regularization parameter. Thus, resulting in a $4^{\text {th }}$ order polynomial fitting model given by: $y_{\lambda}(x)=-0.038 x^{4}+0.12 x^{3}+$ $0.27 x^{2}+0.43 x+0.58$. However, it must be reminded that we could only compute Tab. 4.1 with the knowledge of the exact solution, $y=1.5 x$.

Returning to the review of the theoretical framework, an interpretation of the Bayesian smoothness priors is obtained by multiplying Eq. (4.5) by $\frac{-1}{2 \sigma^{2}}$ and exponentiate (KONISHI; KITAGAWA, 1996). Thus, the unknown that minimizes Eq. (4.5) also maximizes the following relation,

$$
\exp \left(\frac{-1}{2 \sigma^{2}}\|\mathbf{A x}-\mathbf{B}\|^{2}\right) \exp \left(\frac{-\lambda}{2 \sigma^{2}}\|\mathbf{D} \mathbf{x}\|^{2}\right)
$$

where $\sigma^{2}$ remains as the variance of the sensor noise.

From Eq. (4.9), the unbiased solution (since the resulting posterior distribution is assumed Gaussian) is the unknown that maximizes the product of the conditional data distribution $^{3}$ (the first term of Eq. (4.9)) and the prior distribution (the second term of Eq. (4.9)). The main advantage of adopting the Bayesian modelling approach arises from

\footnotetext{
${ }^{3}$ Akaike (1992) introduces the notion that the statistical model may be regarded as depending of the unknown, then it can be called the likelihood function.
} 
the fact that the value of the hyperparameter can be obtained following the approach proposed in (AKAIKE, 1980). From Shibata (1983) and Akaike (1980), it can be shown that when using a finite-dimensional stochastic Gaussian regression model to estimate the assumed infinite-dimensional truth model with a conjugated prior distribution, the ABIC (Akaike's Bayesian Information Criterion) provides an asymptotically efficient solution under the quadratic loss function for selecting the best fit to the data.

Thus, the Bayes theorem (see Eq. (4.2)) is obtained from combining Eq. (4.9) and Eq. (4.3),

$$
P\left(\mathbf{x} \mid \mathbf{B}, \sigma^{2}, \lambda\right) \propto P\left(\mathbf{B} \mid \mathbf{x}, \sigma^{2}\right) p\left(\mathbf{x} \mid \sigma^{2}, \mathbf{D}, \lambda\right)
$$

Since the left hand side of Eq. (4.10) is a proper distribution, the marginal likelihood of the unknown parameters $\left(\lambda\right.$ and $\left.\sigma^{2}\right)$ is given by the integration of the right hand side of the equation,

$$
L\left(\lambda, \sigma^{2}\right)=\iint P\left(\mathbf{B} \mid \mathbf{x}, \sigma^{2}\right) p\left(\mathbf{x} \mid \sigma^{2}, \mathbf{D}, \lambda\right) \mathrm{d} \mathbf{x} \mathrm{d} \mathbf{D}
$$

Good (2003) referred to the maximization of Eq. (4.11) as a type II maximum likelihood method (which includes certain hypotheses concerning the distribution of the data and the use of the proper conjugate prior distribution). An essential result in Akaike (1980) is that, since the posterior distribution is normally distributed, then the marginal likelihood of the unknown parameters can be expressed in a closed form, providing a method for the direct computation of the value of the hyperparameter.

A common approach in information criteria and statistical modelling is the use of the Kullback-Leibler Divergence (KL-Divergence) to measure how the estimated probability distribution diverges from the expected probability distribution. This index is similar to the Fisher information distance (see Walker (2016)) and relies on the expected differential entropy between prior and posterior distributions. In fact, the KL-Divergence can be used to show that the value of the hyperparameter, $\lambda$, estimated with the ABIC minimizes the norm of the difference between the entropy ${ }^{4}$ of the prior and the posterior distributions.

\footnotetext{
${ }^{4}$ The entropy of a system is defined as the minimal amount of information needed to describe its state (AKAIKE, 1992).
} 
This means that the prior distribution obtained using the ABIC is the least informative as well as the one that presents smaller differences with the posterior distribution.

\subsection{Data distributions}

The concepts and hypotheses presented in the previous sections provide a general idea regarding the data distribution. However, a detailed analysis is needed to fully understand the repercussion of these assumptions on the output of the inference problem. A brief review of the two main assumptions and their implications is provided herein, so that the reader can asses the adequacy of these assumptions to the real phenomenon when it is analyzed later in this section:

- Measurement noise is Gaussian: The assumption that the measurement noise is Gaussian is the strongest condition regarding the structure and output of the problem. However, it is also a weak hypothesis as we will see later. Since the difference between the measurements and the estimations is assumed Gaussian with variance, $\sigma^{2}$, and zero mean, this hypothesis leads to a Gaussian Likelihood function of the data model with similar properties.

- Gaussianity of the posterior distribution and conjugate prior distribution: In order to ensure that the posterior distribution is Gaussian, the prior distribution is chosen to be Gaussian (the Gaussian family is conjugate to itself, or self-conjugate, with respect to a Gaussian likelihood function). The fact that the posterior distribution is Gaussian provides a key advantage, because it ensures that the ABIC provides the optimal values for the hyperparameters (KONISHI; KITAGAWA, 1996).

It is widely known, see for example (HAVER; MOAN, 1983), (NIELSEN, 2005) or (FALTINSEN, 1993), that the hypotheses above can be largely affected by the uncertainties related to the stochastic modelling of the ocean waves and the linear wave-body interaction modelling adopted to describe the motions of the platform. Concerning the wave-body interaction modelling procedure, chapter 3 addressed its main characteristics along with the possible shortcomings. Also, it provided a brief description of the stochastic modelling of ocean waves, whose possible drawbacks will be discussed next. 
Basic assumptions commonly adopted for the probabilistic description of ocean waves are: Records (time series) of the process shall be stationary and ergodic in order to estimate meaningful statistical properties from only one realization of the irregular sea. Also, the surface elevation random process is assumed to be Gaussian. These two assumptions are only partially fulfilled in actual sea states. However, they are widely used since they imply very attractive simplifications from a practical point of view.

In addition to these assumptions, uncertainties will be always related to the selection of significant values to represent the sea condition, as for any stochastic phenomenon. These uncertainties should be recognized in the actual learning process since they are somehow included in the measurement noise of the model. For this purpose, following (HAVER; MOAN, 1983) and (BENKE; LOWELL; HAMILTON, 2008), a brief but comprehensive description is provided.

\section{Fundamental uncertainty}

This uncertainty is consequence of making use of stochastic models to characterize the phenomenon in study. Therefore, this uncertainty is inherent to the random nature of the phenomenon.

\section{Model uncertainty}

This uncertainty can be decomposed into two different components: First, as it has been pointed out before, the real nature phenomenon can never be strictly modelled. This means that the analytic model of a physical phenomenon implies simplifications, i.e. an approximated image of the real stochastic phenomenon is considered. Secondly, an uncertainty can be introduced through the selection of the probability distribution for the random variables involved in the phenomenon due to lack of knowledge (if the distribution is known a priori only a reduced model uncertainty is introduced due to the model simplification). If the distribution of the random variable is not known, it is hard to exactly quantify the model uncertainty associated to a specific choice of a probability distribution.

One may realize that this uncertainty is always present and it is implicit to the mathematical model of the physical phenomenon. 


\section{Parameter uncertainty}

(HECKMAN; MEYERS, 1983) is one of the first works that began the analysis of parameter uncertainty. Since the estimation of the parameters has to be done from existing data, there is an uncertainty associated to the estimated variables (subjected to both bias, systematic and, also, random error).

These three uncertainties together with the ones related to the misleading estimations of the RAOs (due to effects discussed in section 3.3) are supposed to be embedded in the measurement noise. As a consequence, one may realize that the assumptions concerning the data distribution of the Bayesian approach adopted may be inaccurate, especially when it comes to the measurement noise. The problem is therefore: Is the measurement noise Gaussian distributed? And if not, what are the implications?

For some sea conditions, specially the more severe sea states, previous studies (HAVER; MOAN, 1983) indicate that sometimes the stationarity assumption can be questioned even for short periods of time (less than $30 \mathrm{~min}$ ). In practice, it is typically assumed that the stationary period holds to few hours (SOARES; FRICKE, 2011). Furthermore, it is widely accepted that a non-Gaussian structure of the sea surface elevation is likely to occur when the wave steepness increases.

In section 3.4 it has been shown that the hypothesis of linear dynamic model for the vessel motions can be questioned for more severe sea states, due to the relatively large steepness of the waves and the possible excitation of resonant motions of the platform, both of which would result in non-Gaussian measurement errors of the stochastic linear regression model adopted. For moderate to mild sea conditions, the RAOs computed in the frequency-domain are expected to provide accurate estimations of the motions of the platform, when combined with the sea spectrum.

In summary, the Gaussianity assumption can be strongly questioned in connection with rough sea states. Therefore, assuming that we have the motion records for a time span for which the sea condition can be assumed stationary, what are the possible effects on the estimation of the sea state using the Bayesian if the Gaussianity assumption is not met or can be questioned? 
For answering this, let us assume that we obtain a certain estimation of the sea condition, namely $\hat{\mathbf{x}}$. Due to the nature of this problem, if the estimator is unbiased, the quadratic loss function has to be minimized as follows,

$$
\min \left\{E\left[(\mathbf{x}-\hat{\mathbf{x}})^{2}\right]\right\}=\min \left\{E\left[\left(\mathbf{x}^{2}-2 \mathbf{x} \hat{\mathbf{x}}+\hat{\mathbf{x}}^{2}\right]\right\}\right.
$$

where $E[\cdot]$ stands for the expected value. Thus, taking the gradient with respect to $\mathbf{x}$,

$$
\nabla\left(E\left[(\mathbf{x}-\hat{\mathbf{x}})^{2}\right]\right)=2 E[\mathbf{x}]-2 E[\hat{\mathbf{x}}]
$$

and setting the gradient equal to zero, the unbiased solution is given $b^{5}$,

\footnotetext{
${ }^{5}$ The reader may have already realized the fact that the main limitation that Eq. (4.14) brings is that it is high-dimensional. For instance, the solution of the problem as it is given in Eq. (4.14) can be computed as follows,

$$
E[\mathbf{x} \mid \mathbf{B}]=\int_{\mathbf{x} \in \Omega} \mathbf{x} \frac{P(\mathbf{x} \mid \mathbf{B}) p(\mathbf{x})}{P(\mathbf{B})} \mathrm{d} \mathbf{x},
$$

where $\Omega$ stands as the space that contains all the possible sea states. Although this space is finite dimensional, its dimensions remain sufficiently large to make the computation of the expected value of $\mathbf{x}$, given certain motion measurements $\mathbf{B}$, unfeasible from a conventional point of view. Nevertheless, there are some approaches that may allow the estimation of the value of this integral, i.e. the expected value of the sea state based on the measured motions, such as the Monte Carlo method for solving high-dimensional integrals (see (SOBOL, 1994)).

Basically, this approach allows the approximation of the integral of any function, let us say for the time being $g(x)$, as the mean value of the ratio $\frac{g(x)}{h(x)}$. Thus,

$$
I=\int_{a}^{b} g(\mathbf{x}) \mathrm{d} x=\int_{a}^{b} \frac{g(\mathbf{x})}{h(\mathbf{x})} h(\mathbf{x}) \mathrm{d} x \approx E\left[\frac{g(\mathbf{x})}{h(\mathbf{x})}\right],
$$
}

where $h(\mathbf{x})$ is any arbitrary probability density function that is defined over the domain of integration, e.g. $1 /(b-a)$. Accordingly, the value of the integral can be approximated as,

$$
I \approx \frac{b-a}{N} \sum_{i=1}^{N} g\left(\mathbf{x}_{i}\right)
$$

This integration method can be generalized to compute multidimensional integrals, such as,

$$
I=\int_{\mathbf{x} \in \Omega} g(\mathbf{x}) \mathrm{d} x
$$

Thus,

$$
I=\int_{\mathbf{x} \in \Omega} \frac{g(\mathbf{x})}{h(\mathbf{x})} h(\mathbf{x}) \mathrm{d} x \rightarrow I=\frac{1}{N} \sum_{i=1}^{N} \frac{g(\mathbf{x})}{h(\mathbf{x})} .
$$

Therefore this method involves the estimation of the values of $g$ (in our case $\frac{\mathbf{x} P(\mathbf{x} \mid \mathbf{B}) p(\mathbf{x})}{P(\mathbf{B})}$ ) at a number $N$ of points, which can be selected randomly in the domain of integration (the result becomes exact when $N \rightarrow \infty$, being the error proportional to $\left.\frac{1}{\sqrt{N}}(\mathrm{SOBOL}, 1994)\right)$. In order to compute the values of this functional a straightforward approach would be the use of the metocean data. Thus for each possible sea state, $\mathbf{x}$, shown by this set of data, it is possible to compute the probability of existence, $p(\mathbf{x})$, by taking the ratio between the number of observed events $(\mathbf{x})$ over the possible number of sea states (in this cases 


$$
\hat{\mathbf{x}}=E[\mathbf{x}]
$$

It is worth remembering that the sea estimation obtained from the Bayesian inference method proposed above is the sea condition that maximizes the posterior distribution. Therefore, if the posterior distribution is Gaussian, i.e. the measurement noise is Gaussian, the expected value is equal to the Mode of the distribution and, as a consequence of Eq. (4.14), the estimation is unbiased. However, if the measurement noise is not Gaussian, even though the optimal value of the hyperparameter is known somehow, the solution obtained by the maximization of the product of the prior distribution and likelihood function is expected to be biased. Furthermore, in this condition the values of the hyperparameters estimated by means of the ABIC approach may not be optimal (ISEKI, 2012).

The resulting bias associated to the estimation in cases when there is a lack of Gaussianity can hardly be quantified in advance. A common procedure adopted in order to overcome this drawback is the use of the Maximum Likelihood Estimation (MLE) procedure, which is equivalent to the use of the Laplace distribution as a prior distribution (KITAGAWA; GERSCH, 2012). The output of this approach, that minimizes the $L_{1}$ norm, is less sensitive to the dispersion of the posterior probability distribution of the variable of interest. Although (in theory) this approach is optimal, it does not provide a proper methodology to choose the optimal values of the hyperparameters.

Returning to the VMB wave inference approach, the results obtained in previous works, such as in (HIRAYAMA, 1987), show that the adoption of the use of the MLE procedure has some limitations regarding the estimation of wave frequency and direction. Otherwise, there are several works that assume that the measurement noise is Gaussian, e.g. (ISEKI; OHTSU, 2000; TANNURI et al., 2003; NIELSEN, 2008; SIMOS et al.,

$p(x)$ would be given by the total number of sea states assessed in the metocean report). It is important to highlight that $h(\mathbf{x})$ can be estimated following the same approach, thus $h(\mathbf{x})=p(\mathbf{x})$. Regarding the likelihood function, $P(\mathbf{x} \mid \mathbf{B})$, it can be computed following (AKAIKE, 1980) (see Eq. (4.22)). Finally, the term $P(B)$ can be easily computed as $\sum_{i=1}^{N} P\left(\mathbf{x}_{i} \mid \mathbf{B}\right) p\left(\mathbf{x}_{i}\right)$.

Unfortunately, this process cannot be adopted for the general purpose of this thesis. This is mainly due to the fact that it relies on the availability of previous metocean data of the sea being monitored. Its main limitation, thus, not only entails the availability of previous metocean data of the field where the campaign is carried out, but also it must be sufficiently representative in order to include a wide range of possible events, i.e. metocean data based on short field measurement campaigns may not include infrequent sea states. Finally, although this methodology stands as a fast procedure for surveying the sea state based on the ship motions, its research and application have been left as a future work. 
2012) and (BISPO et al., 2016). Even though problems may become relevant in some sea condition, such as when part of the high frequency components of the sea spectrum are filtered by the ship (BISPO, 2011b), these works have attested that good estimations of the sea conditions can be obtained, both in model and full-scale applications. Consequently, from now on, the hypothesis of the Gaussian property for the measurement noise will be accepted for the semisubmersible platform, although sometimes at the expense of the optimal solution. Moreover, the improvements made on the RAOs regarding the non-linear effects, discussed later in chapter 5, should favor the validity of the Gaussianity hypothesis. Finally, it should be stressed that the Gaussianity hypothesis of the measurement noise can be considered almost unavoidable (CLAUSS et al., 2014) due to practical reasons related to numerical and/or experimental limitations when it comes to deal with the wave-body interaction problem.

Under this Gaussianity hypothesis, the likelihood function can be written as it is shown next. Also, the formal description of the problem provided ahead is expected to help the reader to fully understand the computation procedure of the Bayesian inference approach, as it is adopted in the present work.

Assuming that the relation between the waves and the platform responses is linear, the directional wave spectrum, $S_{\eta \eta}(\omega, \beta)$, and the vessel RAOs are related to the cross spectra derived from the time series of the platform motions $\left(S_{i j}\right)$ through the following identity (see section 3.3),

$$
S_{i j}(\omega)=\int_{-\pi}^{\pi} \mathrm{Z}_{i}(\omega, \beta) \mathrm{Z}_{j}^{*}(\omega, \beta) S_{\eta \eta}(\omega, \beta) \mathrm{d} \beta,
$$

where $\mathrm{Z}_{i}(\omega, \beta)$ is the Response Amplitude Operator of the motion $i$ at wave frequency $\omega$ and direction of incidence $\beta . \mathrm{Z}_{j}^{*}(\omega, \beta)$ stands for the complex conjugate of the RAO. The relation given in Eq. (4.15) can be rewritten in discrete form assuming the integrand to be constant in each slice $\Delta \beta=\frac{2 \pi}{K}$, leading to,

$$
S_{i j}(\omega)=\Delta \beta \sum_{k=1}^{K} \mathrm{Z}_{i}\left(\omega, \beta_{k}\right) \mathrm{Z}_{j}^{*}\left(\omega, \beta_{k}\right) S_{\eta \eta}\left(\omega, \beta_{k}\right),
$$

where $\beta_{k}=-\pi+(k-1) \Delta \beta$ and $K$ is the number of discrete wave directions considered. Returning to the indices $i$ and $j$, they correspond to the dofs assessed; i.e. if only three 
dofs are adopted $(\mathrm{N}=3)$ : heave, roll and pitch; these indices will vary between 3 and 5 .

If a certain range of $M$ wave frequencies is specified previously, being $\Delta \omega=\frac{\omega_{M}-\omega_{1}}{M-1}$, Eq. (4.16) can be approximated in matrix form as follows,

$$
\mathbf{B}=\mathbf{A} \mathbf{x}+\varepsilon
$$

Here, the vector $\mathbf{B}$ contains a total of $\left(N^{2} \cdot M \times 1\right)$ elements, which are related to the spectra, $S_{i i}$, and the cross-spectra, $S_{i j}$, of the measured motions, and can be expressed as,

$$
\mathbf{B}=\left[\begin{array}{c}
\mathbf{b}_{1} \\
\vdots \\
\mathbf{b}_{m} \\
\vdots \\
\mathbf{b}_{M}
\end{array}\right], \quad \text { with } \quad \mathbf{b}_{\mathbf{m}}=\left[\begin{array}{c}
S_{i i}\left(\omega_{m}\right) \\
\vdots \\
\operatorname{Re}\left[S_{i j}\left(\omega_{m}\right)\right] \\
\vdots \\
\operatorname{Im}\left[S_{i j}\left(\omega_{m}\right)\right] \\
\vdots
\end{array}\right] \text { and } j \geq i
$$

where Re and Im stand for the real and imaginary components, respectively ${ }^{6}$.

The RAO matrix is represented by the matrix $\mathbf{A}$, which is formed by a diagonal of $M$ matrices,

$$
\mathbf{A}=\left[\begin{array}{cccccc}
\mathbf{A}_{1} & 0 & 0 & \cdots & 0 & 0 \\
0 & \mathbf{A}_{2} & 0 & \cdots & 0 & 0 \\
0 & 0 & \ddots & \cdots & 0 & 0 \\
0 & 0 & 0 & \mathbf{A}_{m} & 0 & 0 \\
0 & 0 & 0 & 0 & \ddots & \vdots \\
0 & 0 & 0 & 0 & \cdots & \mathbf{A}_{M}
\end{array}\right]
$$

each of $\left(N^{2} \cdot M\right) \times(K \cdot M)$ elements, and with 0 representing the $\left(N^{2} \times K\right)$ matrix with null elements. The matrices forming the diagonal matrices of $\mathbf{A}$ are given by,

\footnotetext{
${ }^{6} \mathrm{By}$ the calculation of $S$ it is observed that the cross-spectrum is Hermitian, i.e. $S_{i j}=\bar{S}_{j i}$. Hence, only the upper triangular matrix is used, and the resulting number of elements in $\mathbf{b}_{m}$ is given by $N+2\left(\frac{N^{2}-N}{2}\right)=$ $N^{2}$.
} 


$$
\mathbf{A}_{m}=\left[\begin{array}{ccccc}
\left|\mathrm{Z}_{i}\left(\omega_{m}, \beta_{1}\right)\right|^{2} & \cdots & \left|\mathrm{Z}_{i}\left(\omega_{m}, \beta_{k}\right)\right|^{2} & \cdots & \left|\mathrm{Z}_{i}\left(\omega_{m}, \beta_{K}\right)\right|^{2} \\
\vdots & & \vdots & & \vdots \\
\operatorname{Re}\left[\mathrm{Z}_{i}\left(\omega_{m}, \beta_{1}\right) \mathrm{Z}_{j}^{*}\left(\omega_{m}, \beta_{1}\right)\right] & \cdots & \operatorname{Re}\left[\mathrm{Z}_{i}\left(\omega_{m}, \beta_{k}\right) \mathrm{Z}_{j}^{*}\left(\omega_{m}, \beta_{k}\right)\right] & \cdots & \operatorname{Re}\left[\mathrm{Z}_{i}\left(\omega_{m}, \beta_{K}\right) \mathrm{Z}_{j}^{*}\left(\omega_{m}, \beta_{K}\right)\right] \\
\vdots & & \vdots & & \vdots \\
\operatorname{Im}\left[\mathrm{Z}_{i}\left(\omega_{m}, \beta_{1}\right) \mathrm{Z}_{j}^{*}\left(\omega_{m}, \beta_{1}\right)\right] & \cdots & \operatorname{Im}\left[\mathrm{Z}_{i}\left(\omega_{m}, \beta_{k}\right) \mathrm{Z}_{j}^{*}\left(\omega_{m}, \beta_{k}\right)\right] & \cdots & \operatorname{Im}\left[\mathrm{Z}_{i}\left(\omega_{m}, \beta_{K}\right) \mathrm{Z}_{j}^{*}\left(\omega_{m}, \beta_{K}\right)\right] \\
\vdots & & \vdots & & \vdots
\end{array}\right]
$$

Regarding the unknown discretized wave spectrum evaluated at the $(K \times M)$ pairs of frequencies and directions, represented as the vector $\mathbf{x}$ of Eq. (4.17), it is denoted as:

$$
\mathbf{x}=\left[\begin{array}{c}
S_{\eta \eta}\left(\omega_{1}, \beta_{1}\right) \\
S_{\eta \eta}\left(\omega_{1}, \beta_{2}\right) \\
\vdots \\
S_{\eta \eta}\left(\omega_{M}, \beta_{K-1}\right) \\
S_{\eta \eta}\left(\omega_{M}, \beta_{K}\right)
\end{array}\right]
$$

Finally, $\boldsymbol{\varepsilon}$ is a vector with $\left(M \cdot N^{2} \times 1\right)$ elements representing the measurement noise, which has been assumed to be Gaussian, with zero mean value and variance $\sigma^{2}$.

The resulting equation system (see from Eq. (4.18) to (4.21)) will in general be underdetermined. As a matter of fact, typically three degrees of freedom are adopted (namely heave, roll and pitch) while $K=72$ and the number frequencies assessed is equal to $M=20$. Basically, this means that the resulting equation system is composed by $N^{2} \cdot M=$ 180 equations with $72 \cdot 20=1440$ unknowns, thus reflecting a highly under-determined equation system ${ }^{7}$. In this thesis, as well as in other works in the literature (see, for instance (TANNURI et al., 2003)), the adoption of the Bayesian inference approach guarantees a stable solution.

Following Akaike (1980), the Bayes procedure can be applied to the singular system presented by the Eq. (4.17). With such procedure, the estimation of the vector $\mathbf{x}$ is obtained maximizing the product of the likelihood function $L(\mathbf{x} \mid \mathbf{B})$ by the prior dis-

\footnotetext{
${ }^{7}$ One may think that the reduction of the number of directions assessed (e.g. in virtue of the vessel symmetry) and the inclusion of more dofs will result, at some point, in a system with a single unique solution. However, the adoption of more degrees of freedom leads to $\mathbf{A}$ matrices characterized by smaller determinants (when compared with the ones obtained with a small number of dofs) and, therefore, resultoing in problem that are not well-posed.
} 
tribution, where the likelihood function represents the probability of occurrence of the spectrum given an specific measurement, $\mathbf{B}$.

As pointed out above, the measurement noise is assumed Gaussian with zero mean and variance $\sigma^{2}$. Therefore, the likelihood function is given by (AKAIKE, 1980),

$$
L(\mathrm{x} \mid \mathbf{B})=\left(\frac{1}{2 \pi \sigma^{2}}\right)^{\frac{N^{2}}{2}} \exp \left(-\frac{1}{2 \sigma^{2}}\|\mathbf{B}-A \mathbf{x}\|^{2}\right)
$$

\subsection{Prior distributions}

As it has been shown before, see Eq. (4.9), the posterior distribution of the variable of interest is given by the product of the likelihood function and the prior distribution. The following section aims to provide a brief introduction to the different prior distributions previously adopted in the VMB wave inference method. Moreover, in this section the proposal of a new prior distribution is presented, which not only aims to improve the accuracy of the method but also the reduction of the computing time. This alternative approach addresses the VMB wave inference problem by means of the ABIC and the use of heuristic restrictions to solve the optimization problem. Regarding the performance of this alternative proposal, it is assessed by means of a comprehensive comparison with the outputs of the prior distribution already adopted in other works of the group of USP, i.e. (TANNURI et al., 2003; SIMOS et al., 2010; BISPO et al., 2016) and (BISPO, 2011b), and that is adopted in this work as the reference benchmark for the VMB Bayesian wave inference approach. ${ }^{8}$

\footnotetext{
${ }^{8}$ The goodness of the prior distribution can also be assessed as follows. Consider $G$ as the probability distribution function that generates the data as the exact model or the true distribution, and $P(\mathbf{x} \mid \mathbf{B})$ is the estimated model. Then, using Eq. (4.10) the KL-Divergence is given by,

$$
\mathrm{I} \propto E_{G}\left[\log \frac{G}{P(\mathbf{B} \mid \mathbf{x})}-\log \frac{P(\mathbf{x} \mid \mathbf{B})}{p(\mathbf{x})}\right] .
$$

The two terms in the right side of Eq. (8) can be interpreted respectively as an accuracy term, given by the logarithm of the generating function over the posterior expected likelihood, and a complexity term, obtained by the logarithm of the ratio of the posterior and the prior distribution. This means that models with likelihood functions and prior distributions close to the real generating function and the posterior distribution, respectively, receive low penalties. The relation given by the previous equation provides a tool to select models that reproduce observations well (high accuracy) without including too much prior information.
} 


\subsubsection{Conventional approach}

The non-parametric approach adopted in this study follows the Bayesian modelling procedure as proposed by Akaike (1980), which essentially aims at introducing various kinds of prior information regarding the directional wave spectrum to improve the solution of the ill-conditioned problem as well as to reduce the influence of the noise. In wave inference, the prior information essentially concerns the fact that the spreading of wave energy should happen smoothly (in frequency and direction). Mathematically this restraint can be translated, among other possibilities, into delimiting the curvature of the function as it shown in the following. For this aim, let us assume that the value of the spectrum at direction, $\beta_{k}$, and frequency, $\omega_{i-1}$, can be estimated as,

$$
S_{\eta \eta}\left(\omega_{i+1}, \beta_{k}\right)=S_{\eta \eta}\left(\omega_{i}, \beta_{k}\right)+\Delta \omega S_{\eta \eta}^{\prime}\left(\omega_{i}, \beta_{k}\right)+\frac{\Delta \omega^{2}}{2} S_{\eta \eta}^{\prime \prime}\left(\omega_{i}, \beta_{k}\right)+\vartheta\left(\Delta \omega^{3}\right)
$$

where $S_{\eta \eta}^{\prime \prime}\left(\omega_{i}, \beta_{k}\right)$ stands as the second order derivative of the wave spectrum with respect to the variable of interest, in this case $(\omega)$. Following a similar approach, the spectrum can be evaluated at the pair of frequency and direction, $\omega_{i+1}$ and $\beta_{k}$, obtaining that,

$$
S_{\eta \eta}\left(\omega_{i-1}, \beta_{k}\right)=S_{\eta \eta}\left(\omega_{i}, \beta_{k}\right)-\Delta \omega S_{\eta \eta}^{\prime}\left(\omega_{i}, \beta_{k}\right)+\frac{\Delta \omega^{2}}{2} S_{\eta \eta}^{\prime \prime}\left(\omega_{i}, \beta_{k}\right)+\vartheta\left(\Delta \omega^{3}\right),
$$

Now, assume that we have two different models whose KL-Divergences are given by $\mathrm{I}=\mathrm{I}(G ; P(\mathbf{x} \mid \mathbf{B}))$ and $\mathrm{I}^{*}=\mathrm{I}\left(G ; P^{*}(\mathbf{x} \mid \mathbf{B})\right)$. Thus,

$$
\Delta I=\mathrm{I}-\mathrm{I}^{*}=E_{G}\left[\log \frac{G}{P(\mathbf{B} \mid \mathbf{x})}-\log \frac{G}{P^{*}(\mathbf{B} \mid \mathbf{x})}+\log \frac{P(B)}{p(\mathbf{x})}-\log \frac{P^{*}(B)}{p^{*}(\mathbf{x})}\right],
$$

It is expected that the output of the likelihood function of both models is approximately equal. Although this is a strong assumption, it is known that there are several sea conditions that provide analogous minimum values for the likelihood function. Accordingly, it is accurate enough to assume that the expected value of the likelihood is approximately equal for both models, leading to:

$$
\Delta I \propto E_{G}\left[\log \frac{P(\mathbf{B})}{p(\mathbf{x})}-\log \frac{P^{*}(\mathbf{B})}{p^{*}(\mathbf{x})}\right] .
$$

If the estimation $P(\mathbf{x} \mid \mathbf{B})$ is more accurate than $P^{*}(\mathbf{x} \mid \mathbf{B})$ then $\Delta I<0$. Therefore the prior distribution of the first model is less informative than the second one. The relation obtained using a measurement of the entropy between the posterior distribution and the prior distribution ensures, for the cases where two different estimations are obtained for the same sea condition, that the improvement of the estimations of the sea condition are directly related to the prior distribution selected. 
Combining Eqs. (4.23) and (4.24), it is possible to estimate the value of the spectrum at the pair $\left(\omega_{i}, \beta_{k}\right)$,

$$
S_{\eta \eta}\left(\omega_{i}, \beta_{k}\right)=\frac{1}{2}\left(S_{\eta \eta}\left(\omega_{i-1}, \beta_{k}\right)+S_{\eta \eta}\left(\omega_{i+1}, \beta_{k}\right)\right)+\frac{\Delta \omega^{2}}{2} S_{\eta \eta}^{\prime \prime}\left(\omega_{i}, \beta_{k}\right)+\vartheta\left(\Delta \omega^{3}\right),
$$

This approximation is possible due to the fact that the smoothness condition in frequency and direction domain can be introduced separately in the prior distribution, as it is shown later. Furthermore, the same procedure can be followed to introduce the smoothness condition in the direction domain. Returning to the model proposed to estimate the value of the spectrum at the pair $\left(\omega_{i}, \beta_{k}\right)$, it is commonly reduced to the truncated form of Eq. (4.25), as it is shown in Eq. (4.26).

$$
S_{\eta \eta}\left(\omega_{i}, \beta_{k}\right)=\frac{1}{2}\left(S_{\eta \eta}\left(\omega_{i-1}, \beta_{k}\right)+S_{\eta \eta}\left(\omega_{i+1}, \beta_{k}\right)\right)
$$

However, when it comes to describe the behavior of the spectrum, the model provided in Eq. (4.26) may present some shortcomings; due to the fact that it provides an approximation of the exact value that the spectrum presents at $\left(\omega_{i}, \beta_{k}\right)$. Therefore, an uncertainty regarding the prior belief (Eq. (4.26)) is accepted,

$$
S_{\eta \eta}\left(\omega_{i}, \beta_{k}\right)=\frac{1}{2}\left(S_{\eta \eta}\left(\omega_{i-1}, \beta_{k}\right)+S_{\eta \eta}\left(\omega_{i+1}, \beta_{k}\right)\right)+\varepsilon_{p}
$$

where $\varepsilon_{p}$ represents the uncertainty of the prior model and stands as an approximation of the high order terms $\left(\frac{\Delta \omega^{2}}{2} S_{\eta \eta}^{\prime \prime}\left(\omega_{i}, \beta_{k}\right)\right.$ and $\left.\vartheta\left(\Delta \omega^{3}\right)\right)$ of Eq. (4.25). Regarding the main properties of this uncertainty, it has been assumed to be Gaussian with zero mean and variance $\sigma_{p}^{2}$. Intuitively, the standard deviation determines how much the reconstructed function (unknown spectrum) departs from a line. In terms of matrices we obtain,

$$
\varepsilon_{p}=\mathbf{H} \mathbf{S}_{\eta \eta}\left(\omega, \beta_{k}\right)
$$

where $S_{\eta \eta}\left(\omega, \beta_{k}\right)$, in this case, is the wave spectrum for a fixed direction, $k$, and $H$ can be directly deducted by identification from Eq. (4.27), 


$$
\mathbf{H}=\left[\begin{array}{cccccccc}
1 & -2 & 1 & 0 & \cdots & 0 & 0 & 0 \\
0 & 1 & -2 & 1 & \cdots & 0 & 0 & 0 \\
\vdots & \vdots & \vdots & \vdots & \ddots & \vdots & \vdots & \vdots \\
0 & 0 & 0 & 0 & \cdots & 1 & -2 & 1
\end{array}\right]
$$

which stands as an approximation of the second order finite difference matrix. However, this matrix has a rank equal to $M-1$, that leads to a prior distribution that is a degenerated Gaussian density function in $\mathbb{R}^{M+1}$. This is due to the absence of boundary conditions for $S_{\eta \eta}(\omega, \beta)^{9}$. A possible option, as proposed by Nielsen (2006), is to assume that in the direction domain the following relation is fulfilled: $S_{\eta \eta}\left(\omega_{m}, \beta_{0}\right)=S_{\eta \eta}\left(\omega_{m}, \beta_{k}\right)$ and $S_{\eta \eta}\left(\omega_{m}, \beta_{K+1}\right)=S_{\eta \eta}\left(\omega_{m}, \beta_{1}\right)$. Regarding the frequency-domain, the condition imposed is that the power of the spectrum is minimized for $\omega \rightarrow 0$ and $\omega \rightarrow \infty$.

This central idea was used for the first time in (ISEKI; OHTSU, 2000) to estimate the wave spectrum from the motions measured on a ship with forward speed. In their approach, the trade-off between the good fit to the data and the smoothness of the solution is then controlled by means of a single hyperparameter, $u=\frac{\sigma}{\sigma_{p}}$. In this case, the posterior distribution is given by,

$$
L(\mathbf{x} \mid \mathbf{B}) \cdot p(\mathbf{x})=\left(\frac{1}{2 \pi \sigma^{2}}\right)^{\frac{N^{2}}{2}} \exp \left(-\frac{1}{2 \sigma^{2}}\left[\|\mathbf{B}-\mathbf{A x}\|^{2}+\mathbf{x}^{T}\left(u^{2}\left(\mathbf{H}_{1}+\mathbf{H}_{2}+\mathbf{H}_{3}\right)\right) \mathbf{x}\right]\right)
$$

where $\mathbf{H}_{1}$ and $\mathbf{H}_{2}$ stand for the discrete second order derivatives of the estimated vector, that can be easily computed considering the definition of vector $\mathbf{x}$ (NIELSEN, 2008), as it will be shown next.

The smoothness condition can be introduced through an approximation of the second order differences associated to the estimated spectrum (see Eqs. (4.27) and (4.29)) in direction, $\varepsilon_{1 m k}$, and frequency, $\varepsilon_{2 m k}$, that are simply defined as,

\footnotetext{
${ }^{9} \mathrm{~A}$ brief discussion concerning the boundary conditions is provided in appendix E.
} 


$$
\begin{aligned}
& \varepsilon_{1 m k}=S_{\eta \eta}\left(\omega_{m}, \beta_{k-1}\right)-2 S_{\eta \eta}\left(\omega_{m}, \beta_{k}\right)+S_{\eta \eta}\left(\omega_{m}, \beta_{k+1}\right) \\
& \varepsilon_{2 m k}=S_{\eta \eta}\left(\omega_{m-1}, \beta_{k}\right)-2 S_{\eta \eta}\left(\omega_{m}, \beta_{k}\right)+S_{\eta \eta}\left(\omega_{m+1}, \beta_{k}\right) .
\end{aligned}
$$

Also, it has been pointed out that the prior distribution aims at guaranteeing an estimated sea spectrum with a certain degree of smoothness. This condition is equivalent to keeping the following relations as small as possible (see Eq. (4.27) and Eq. (4.28)),

$$
\begin{aligned}
& \sum_{k=1}^{K} \sum_{m=1}^{M} \varepsilon_{1 m k}=\mathbf{x}^{T} \mathbf{H}_{1} \mathbf{x}, \\
& \sum_{k=1}^{K} \sum_{m=1}^{M} \varepsilon_{2 m k}=\mathbf{x}^{T} \mathbf{H}_{2} \mathbf{x} .
\end{aligned}
$$

where $S_{\eta \eta}\left(\omega_{m}, \beta_{0}\right)=S_{\eta \eta}\left(\omega_{m}, \beta_{K}\right)$ and $S_{\eta \eta}\left(\omega_{m}, \beta_{K+1}\right)=S_{\eta \eta}\left(\omega_{m}, \beta_{1}\right)$.

In addition, one must take care of another practical problem regarding the limitations due to the frequency insensitivity already mentioned in section 3.3, i.e. wave frequencies for which part or all the motions of the platform are null. As a consequence, any estimation in this frequency range therefore not supported by a proportionality between excitation and response. In order to avoid an over prediction of the spectrum energy leading to inconsistent results, the total energy of the spectrum is minimized in two pre-defined ranges of low and high frequencies. This can be reached by minimizing the following expression,

$$
\sum_{k=1}^{K} \sum_{m=1}^{L} S_{\eta \eta}\left(\omega_{m}, \beta_{k}\right)^{2}+\sum_{k=1}^{K} \sum_{m=H}^{M} S_{\eta \eta}\left(\omega_{m}, \beta_{k}\right)^{2}=\mathbf{x}^{T} \mathbf{H}_{3} \mathbf{x}
$$

when $\omega_{L}$ and $\omega_{H}$ are respectively the upper and lower frequencies of the range whose energy is to be minimized.

Accordingly, the posterior distribution in Eq. (4.30) can be derived using the relations provided above. Moreover, it is worth remembering that the sea condition that minimizes the relations in Eq. (4.32) and Eq. (4.33) is the same sea spectrum that would maximize the product of the likelihood function, Eq. (4.22), and the prior distribution, given in Eq. (4.30). 
Following the idea presented in (ISEKI; OHTSU, 2000), Nielsen (2008) proposed the introduction of an additional hyperparameter, thus leading to a prior distribution with two hyperparameters. In the resulting distribution, the first hyperparameter, $u_{1}$, determines the balance of the fit of the model and the smoothness in direction while the second one, $u_{2}$, controls the smoothness of the energy distribution regarding the wave frequency. The effects of $\mathbf{H}_{3}$ on the final solution are addressed in (NIELSEN, 2008) by means of the minimization of the sum of the power spectrum in the frequency range where the ship presents no significant motions. Therefore, the optimal solution in (NIELSEN, 2008) is obtained by maximizing the following functional:

$$
L(\mathbf{x} \mid \mathbf{B}) \cdot p(\mathbf{x})=\left(\frac{1}{2 \pi \sigma^{2}}\right)^{\frac{N^{2}}{2}} \exp \left(-\frac{1}{2 \sigma^{2}}\left[\|\mathbf{B}-\mathbf{A} \mathbf{x}\|^{2}+\mathbf{x}^{T}\left(u_{1}^{2} \mathbf{H}_{1}+u_{2}^{2} \mathbf{H}_{2}\right) \mathbf{x}\right]\right)
$$

Common to these previous applications of VMB wave inference is the fact that the determination of the optimal values of the hyperparameters has been done by minimizing the ABIC, i.e. following the procedure proposed in Akaike (1980), despite the expensive computational cost that is required by this methodology.

Other works in the field (see for example Tannuri et al. (2003) or Simos et al. (2012)) used an approach based on the one proposed by Nielsen (2008), but including a third hyperparameter, $u_{3}$, which controls the excessive energy estimation at frequency boundaries. Nonetheless, the main modification proposed in Tannuri et al. (2003) and adopted in subsequent works of the group of University of São Paulo, concerns the estimation of the hyperparameters, which are pre-defined in their apporach. As a matter of fact, the use of pre-defined hyperparameters increases the functionality of the method, thus, resulting in a less time-consuming approach that may be adopted in applications that rely on real-time data, such as decision support systems for offshore operations. Therefore, in this case, the statistical approach, followed to determine the hyperparameters, has been abandoned by these authors to increase the practical applicability of the method (TANNURI et al., 2003). Regarding the calibration procedure of the (pre-defined) hyperparameters, it has been improved over the time by means of sensitivity studies carried out using model-scale and full-scale data, as it shown in (SIMOS et al., 2010; BISPO et 
al., 2012) and (SIMOS et al., 2012; BISPO et al., 2016; BISPO, 2011b), respectively. In this approach the posterior distribution is hence written as,

$$
L(\mathbf{x} \mid \mathbf{B}) \cdot p(\mathbf{x})=\left(\frac{1}{2 \pi \sigma^{2}}\right)^{\frac{N^{2}}{2}} \exp \left(-\frac{1}{2 \sigma^{2}}\left[\|\mathbf{B}-\mathbf{A} \mathbf{x}\|^{2}+\mathbf{x}^{T}\left(u_{1}^{2} \mathbf{H}_{1}+u_{2}^{2} \mathbf{H}_{2}+u_{3}^{2} \mathbf{H}_{3}\right) \mathbf{x}\right]\right)
$$

with $u_{1}, u_{2}$ and $u_{3}$ known.

Two main motivations can be underlined among the arguments that lead to pre-define the values of the hyperparameters in (TANNURI et al., 2003) and (SIMOS et al., 2012) as well as in (BISPO, 2011a; SIMOS et al., 2012) and (BISPO et al., 2016). First, since the ABIC method implies multiple testing, the technique followed by Nielsen is very timeconsuming (TANNURI et al., 2003) and considered impractical for real-time applications, such as decision support systems. Secondly, some works, e.g. (ISEKI, 2012), showed that in some cases the results obtained with the ABIC do not provide the optimal solution. The discrepancies observed can be explained in those cases by one of the majors drawbacks of the ABIC method, i.e. is its instability under certain conditions. For example, when the linear model is not sufficient to characterize the sea state (e.g. when non-linear effects start to have some relevance on the ship motions or when the ship has no significant motions, leading to situations in which the Gaussianity assumption of the measurement noise may not be fulfilled), it is usually hard for the method to distinguish solutions that are close to each other. In this case, the choice of the values of the hyperparameters becomes unstable during the multiple testing stage and a non-asymptotic behavior of the solution is reached, leading to erratic and inaccurate estimations of the sea conditions.

Therefore, the approach proposed initially by Tannuri et al. (2003) with the calibration procedure defined in Simos et al. (2012) will be the methodology adopted from now on in this work to assess the use of the semisubmersible platform as a VMB wave sensor and to provide a reference for evaluating the quality of the estimation obtained with the alternative prior distribution proposed in the following section. Finally, it should be noticed that the maximization of the product of the likelihood function and the prior distribution, given in Eq. (4.35), is then equivalent to the minimization of the following functional, 


$$
J(\mathbf{x})=\|\mathbf{B}-\mathbf{A} \mathbf{x}\|^{2}+\mathbf{x}^{T}\left(u_{1}^{2} \mathbf{H}_{1}+u_{2}^{2} \mathbf{H}_{2}+u_{3}^{2} \mathbf{H}_{3}\right) \mathbf{x} .
$$

which is feasible by means of a conventional quadratic algorithm.

\subsubsection{Alternative prior distribution}

This section contains a detailed explanation regarding the proposal of an alternative prior distribution for the Bayesian inference method. Nonetheless, a comprehensive description of the main shortcomings of the approach adopted by Simos et al. (2012) and Bispo et al. (2016) is provided in order to enable the reader to understand the main motivations that led to this proposal.

\section{Limitations of the conventional approach}

As it has been pointed out in chapter 1, the results of ten months of a field campaign on a FPSO platform were used in Bispo et al. (2016). This work highlighted the good accuracy of the Bayesian method to estimate the sea conditions that happened during this period of time provided they were in the estimation range $\left(T_{p}>8 \mathrm{~s}\right)$. However, in some sporadic cases the authors also observed large discrepancies between the estimations obtained and the radar measurements, which were adopted as the basis for assessing the performance of the VMB wave inference method. These discrepancies can be grouped as follows: (i) spurious energy peaks at the bow and stern of the vessel in beam sea conditions and (ii) global mean wave direction, when deviates from the expected value, has a consistent and systematic error.

It is plausible to consider that part of the misleading estimations of the sea conditions can be explained by limitations of the prior distribution adopted. Possible improvements of the prior distribution are:

- It is known from the formal discussion provided in (AKAIKE, 1980), that the prior distribution should be determined taking into account the measured data. Although previous works developed by the research group of USP have been based on a precalibration procedure of the hyperparameters (TANNURI et al., 2003), the current work aims at providing a prior distribution which is computed using the measured data without compromising the practical features gained with the pre-calibration of 
the hyperparameters;

- As mentioned earlier, the likelihood function adopted works as a high-pass filter and it has the undesirable effect of amplifying the measurement uncertainties when the ship is expected to present no significant motions, see Eq. (4.22). Thus, a mechanism capable of properly computing the variance introduced by the points of minima of the RAOs would be welcome;

- The pre-definition of the two first hyperparameters, $\left(u_{1}, u_{2}\right)$, showed several advantages, e.g. the algorithm is less time-consuming and a consistent improvement of the estimation has been observed. However, in some cases the values adopted for the hyperparameters are not optimal (see Bispo (2011a)). This effect may be related to the point of minima of the RAOs, where the vessel is expected to have no significant responses, but certain wave conditions may impose some motions leading to poor estimations of the sea state (see section 3.3.2). Also, the number of hyperparameters and the calibration procedure adopted may not lead to the lower expected mean squared error, as it is shown by the Stein's paradox (STEIN, 1959). In addition, this approach requires a pre-calibration procedure that involves numerical simulations and, in some cases, real scale data to validate the values estimated with the hyperparameters chosen. The novel prior distribution proposed does not rely on the use of these hyperparameters, and therefore it is expected that this characteristic of the prior distribution will help to avoid some of the drawbacks related to the pre-defined hyperparameters;

- Despite the fact that the use of the third hyperparameter provides an useful technique to avoid spurious energy estimations, this methodology restrains the estimation of the energy in certain frequency ranges in which the ship still has representative motions and this can have a negative impact on the estimations of the significant wave height and mean period. Thus, the prior distribution proposed does not rely on the use of a third hyperparameter, $u_{3}$, whose influence on the final solution has been modelled by means of a method that avoids misleading energy estimations within the frequency limits.

From the points listed above, the following can be concluded: For mitigating the errors of estimation, the uncertainties introduced by the RAOs should be properly addressed by 
the prior distribution. Also, a method to calibrate the trade-off between the likelihood function and the prior distribution is needed, at least one that would lead to optimal estimations of the prior distribution in the majority of the cases.

\section{An heuristic prior distribution}

Next, the deduction of an alternative prior distribution that aims to reach the improvements suggested above is presented. This is followed by a detailed description of the benefits expected with the use of this new prior distribution in VMB wave inference problems.

For this purpose, let us assume that the prior distribution function of $S_{\eta \eta}$ is given by a negative exponential probability distribution in the form of,

$$
p(\mathbf{x}) \propto \exp \left\{-\frac{\|\mathbf{C x}\|^{2}}{2 \sigma^{2}}\right\},
$$

where $\mathbf{C}$ stands as a matrix of coefficients that must be estimated. Once again, this assumption is in accordance with the theory presented above (see section 4.2). Furthermore, this assumption allows us to estimate the variance of each element of the unknown spectrum, $S_{\eta \eta_{j}}{ }^{10}$, as follow (see (BUI-THANH, 2012)),

$$
\operatorname{Var}\left(S_{\eta \eta_{j}}\right)=\mathbf{e}_{j}\left(\int_{\mathbb{R}^{M+1}} S_{\eta \eta}^{2} p\left(S_{\eta \eta}\right) \mathrm{d} S_{\eta \eta}\right) \mathbf{e}_{j}^{T}=\sigma^{2} \mathbf{e}_{j}\left(\mathbf{C}^{T} \mathbf{C}\right)^{-1} \mathbf{e}_{j}
$$

where $\boldsymbol{e}_{j}$ stands for the $j^{\text {th }}$ canonical basis ${ }^{11}$ vector in $\mathbb{R}^{M+1}$.

Accordingly,

$$
C_{j j}=\left(\frac{\sigma^{2}}{\operatorname{Var}\left(S_{\eta \eta_{j}}\right)}\right)^{\frac{1}{2}} \text { and } C_{i j}:=0
$$

Let us assume an approximate solution to the problem $(\hat{\mathbf{x}})$ be somehow known. It is possible to obtain the same estimation from Eq. (4.5) setting the matrix containing the different weights of the regularization term $\mathbf{D}$ equal to the $\mathbf{C}$. Now, it has been assumed,

\footnotetext{
${ }^{10}$ In this case $j$ ranges between 1 and $K \cdot M$. Thus, $S_{\eta \eta_{j}}=S_{\eta \eta}\left(\omega_{m}, \beta_{k}\right)$.

${ }^{11}$ The reader is referred to appendix $\mathrm{E}$ for a comprehensive discussion regarding the size of the higherdimensional space of the prior distribution.
} 
heuristically, that the variance of each element of the unknown spectrum is proportional to,

$$
\operatorname{Var}\left(S_{\eta \eta_{j}}\right) \propto \frac{\max (\hat{\mathbf{x}})-\min (\hat{\mathbf{x}})}{\left|\hat{\mathbf{x}}_{j}-\max (\hat{\mathbf{x}})\right|}
$$

Now, because the regularization term can be assimilated to an approximation of the measurement noise, as shown by the result obtained in Eq. (4.39), and aiming to facilitate the computing process, the final expression of each element of matrix $\mathbf{C}$ has been adopted as,

$$
C_{j j} \cdot C_{j j}=\frac{\left|\hat{\mathrm{x}}_{j}-\max (\hat{\mathbf{x}})\right|}{\max (\hat{\mathbf{x}})-\min (\hat{\mathbf{x}})} \sigma^{2},
$$

This results in a regularization term that induces larger estimation errors in the frequency regions in which the sea spectrum presents small amounts of power or the responses of the platform are not significant, which is in accordance with the results obtained by means of the coherence functions (discussed in section 5.4.2), i.e. the uncertainty of the linear model is larger when the platform does not present significant motions.

From Eq. (4.41), the computation of the matrix $\mathbf{C}$ is reduced to the estimation of the approximation of the sea spectrum $\hat{\mathbf{x}}$, that is also needed to estimate the $j^{\text {th }}$ element of the variance of the measurement noise, $\sigma^{2}$, as it is shown below.

Therefore, the measured data will be used to compute the prior distribution. It is known that the use of the measured data to construct the prior distribution can introduce different correlations between the likelihood function and the prior distribution, resulting in misleading estimations of the sea conditions. Aiming to avoid any bias on the posterior distribution, as well as to maintain the benchmark of the prior distribution proposed in previous works, the ABIC method has been adopted.

Introducing the posterior distribution obtained by Nielsen (2008), see Eq. (4.9), in the general form of the ABIC (AKAIKE, 1980), that is given by,

$$
A B I C=-2 \log \int P_{\text {post }}(\mathbf{x} \mid \ldots) \mathrm{d} \mathbf{x}
$$

The following relation for the ABIC can be obtained, 
$A B I C=N(N+1) \log \left(2 \pi \sigma^{2}\right)-K \cdot M \lg \left(\lambda^{2}\right)-2 \log \int\left(\frac{1}{2 \pi \sigma^{2}}\right)^{\frac{K M}{2}} \exp \left\{\frac{-1}{2 \sigma^{2}} J(\hat{\mathbf{x}})\right\}$,

Following the results obtained by Nielsen (2008), Eq. (4.43) can be rearranged as,

$$
A B I C=N(N+1) \log \left(2 \pi \sigma^{2}\right)-K \cdot M \lg \left(\lambda^{2}\right)+\frac{J(\hat{\mathbf{x}})}{\sigma^{2}}+\mathcal{F}(\lambda, \mathbf{A}, \mathbf{D})
$$

where $N, M$ and $K$ are constants related to the number of variables, as it has been pointed out in section 4.3, and $J(\hat{\mathbf{x}})=\|\mathbf{B}-\mathbf{A} \hat{\mathbf{x}}\|^{2}+\hat{\mathbf{x}}^{T}\left(u_{1}^{2} \mathbf{H}_{1}+u_{2}^{2} \mathbf{H}_{2}\right) \hat{\mathbf{x}}$.

In order to compute the value of $\sigma^{2}$ that minimizes the ABIC, Eq. (4.44) is differentiated with respect to the variable of interest $\left(\sigma^{2}\right)$ and then set equal to zero, leading to,

$$
\frac{N(N+1)}{2 \pi \sigma^{2}} 2 \pi-\frac{J(\hat{\mathbf{x}})}{\sigma^{4}}=0
$$

that can be rewritten as,

$$
\sigma^{2}=\frac{J(\hat{\mathbf{x}})}{N(N+1)}=\frac{\|\mathbf{B}-\mathbf{A} \hat{\mathbf{x}}\|^{2}+\hat{\mathbf{x}}^{T}\left(u_{1}^{2} \mathbf{H}_{1}+u_{2}^{2} \mathbf{H}_{2}\right) \hat{\mathbf{x}}}{N(N+1)} .
$$

To compute Eq. (4.46), an estimation of the residual of the regularized objective function, $J(\hat{\mathbf{x}})$, is needed. The objective function selected for this purpose is the one resulting from Eq. (4.34), which guarantees the smoothness and continuity of the solution in frequency and direction. Also, it is compatible with the hypotheses assumed. Since the matrix $\mathbf{C}$ is expected to provide an estimation of the variance of measurement noise, the values of the hyperparameters of Eq. (4.34) were set equal to 1, that also is consistent with Eq. (4.41). Finally, an estimation of $J(\hat{\mathbf{x}})$ is needed in order to compute the variance of the measured noise. Therefore,

$$
\hat{\mathbf{x}}=\left(\mathbf{A}^{T} \mathbf{A}+1^{2} \mathbf{H}_{1}+1^{2} \mathbf{H}_{2}\right)^{-1} A^{T} \mathbf{B} .
$$


Since we are trying to approximate the variance of the measurement noise and Eq. (4.47) provides the minimum of the objective function under the hypotheses adopted, from $\mathrm{Hu}-$ ber $(1965)^{12}$ we obtain that a single estimation of the residual of the objective function is enough, making the process less time-consuming. It is worthy to highlight that the objective function selected will be indirectly included into the prior distribution and, as a consequence, the adoption of this objective function assures a certain preference to smooth solutions. Nonetheless, at the same time, it acts as a weak condition in contrast with the maximization problem proposed by Nielsen (2006).

The resulting procedure may provide large values of $C_{j j}$ for certain frequencies and directions. In order to avoid unbounded values that would result in an unstable prior distribution, the values of the elements of matrix $\mathbf{C}$ will be kept equal to or below a certain threshold, as shown next.

From the Gaussian linear regression model adopted, see Eq. (4.3), it is known that the measurement error is Gaussian distributed with zero mean and variance $\sigma^{2}$. Thus, $\mathbf{x}$ is a normally distributed parameter vector independent of $\varepsilon$ with zero mean and covariance $\sigma_{x}^{2}$

$$
\left[\begin{array}{l}
\mathbf{x} \\
\boldsymbol{\varepsilon}
\end{array}\right]=\mathcal{N}\left(\left[\begin{array}{l}
0 \\
0
\end{array}\right],\left[\begin{array}{cc}
\sigma_{x}^{2} & 0 \\
0 & \sigma^{2}
\end{array}\right]\right) .
$$

From,

$$
\left[\begin{array}{l}
\mathbf{x} \\
\mathbf{B}
\end{array}\right]=\left[\begin{array}{ll}
I & 0 \\
A & I
\end{array}\right]\left[\begin{array}{l}
\mathbf{x} \\
\varepsilon
\end{array}\right],
$$

The following relation is obtained,

$$
E\left[\begin{array}{l}
\mathbf{x} \\
\mathbf{B}
\end{array}\right]=\left[\begin{array}{l}
0 \\
0
\end{array}\right]
$$

and the respective variances can be computed as follows,

\footnotetext{
${ }^{12}$ That work proved consistency and asymptotic normality of the maximization objective function under relatively weak conditions concerning the exact distribution of the likelihood function and the use of the second derivatives of the likelihood function as the regularity conditions.
} 
$E\left[\left[\begin{array}{c}\mathbf{x}-\hat{\mathbf{x}} \\ \mathbf{B}-\hat{\mathbf{B}}\end{array}\right]\left[\begin{array}{l}\mathbf{x}-\hat{\mathbf{x}} \\ \mathbf{B}-\hat{\mathbf{B}}\end{array}\right]^{T}\right]=\left[\begin{array}{cc}I & 0 \\ A & I\end{array}\right]\left[\begin{array}{cc}\sigma_{x}^{2} & 0 \\ 0 & \sigma^{2}\end{array}\right]\left[\begin{array}{cc}I & 0 \\ A & I\end{array}\right]^{T}=\left[\begin{array}{cc}\sigma_{x}^{2} & \sigma_{x}^{2} A^{T} \\ A \sigma_{x}^{2} & A \sigma_{x}^{2} A^{T}+\sigma^{2}\end{array}\right]$.

Therefore,

$$
\begin{aligned}
& \operatorname{Cov}(\mathbf{x}, \mathbf{x})=\sigma_{x}^{2} \\
& \operatorname{Cov}(\mathbf{x}, \mathbf{B})=A \sigma_{x}^{2} \\
& \operatorname{Cov}(\mathbf{B}, \mathbf{B})=\sigma_{B}^{2}=A \sigma_{x}^{2} A^{T}+\sigma^{2}
\end{aligned}
$$

Consequently, the covariance values of the motions would be adopted as the limiting threshold for the values of the elements of matrix $\mathbf{C}$, leading to the following relation,

$$
C_{j j} \cdot C_{j j}=\frac{\left|\hat{\mathbf{x}}_{j}-\max (\hat{\mathbf{x}})\right|}{\max (\hat{\mathbf{x}})-\min (\hat{\mathbf{x}})} \sigma^{2} \leq \sigma_{B}^{2}
$$

An estimation of $\mathbf{C}$ can be obtained by substituting the output of Eq. (4.46) and Eq. (4.47) into Eq. (4.53). However, this procedure, once again, may lead to unbounded values for the estimation of the unknown for the pairs of frequencies and directions linked to the point of minima of the RAOs. As mentioned in chapter 3, the zero points of the RAOs may introduce some uncertainties due to the fact that waves with frequencies and directions that correspond to cancellations points of the RAOs, when the vessel is expected to have no significant response, may, in reality, impose some motions on the platform. This is because the numerical model for the RAOs is not free from errors (as it has been shown in chapter 3). The procedure described below avoids this shortcoming.

1. Components $C_{j j}$ of matrix $\mathbf{C}$ linked to the cancelation points of the RAOs are set equal to the maximum of the output obtained by means of the estimation of matrix C.

2. Also, components of matrix $\mathbf{C}$ near to the cancelation points and points of minima of the RAOs may provide, in certain conditions, unrealistic estimations of the wave spectrum. Therefore, if the corresponding values of $\mathbf{C}$ are less than a certain thresh- 
old, then the values are replaced by this threshold. The estimation of this threshold is done be means of a linear interpolation between the maximum and minimum of matrix $\mathbf{C}$ accordingly to the heave RAO, which has been select among the (six) RAOs due to the fact that it can be considered as an almost ideal response model ${ }^{13}$, thus providing a better prediction of the responses of the platform in comparison with the other dofs.

A formal description of the alternative prior distribution can be deduced introducing the uncertainty, namely $\mathbf{w}$, that the points of minima or cancellation points of the RAOs can generate on the model. Since this uncertainty is unknown at the beginning, it can be included into the inference model using a common approach followed in Bayesian networks, which essentially considers the uncertainty as a parameter of the distribution, obtaining the following equation:

$$
\begin{gathered}
P(\mathbf{x}, \mathbf{w} \mid \mathbf{B}) P(\mathbf{B})=P(\mathbf{x}, \mathbf{w}, \mathbf{B})=P(\mathbf{B} \mid \mathbf{x}, \mathbf{w}) P(\mathbf{x}, \mathbf{w})= \\
P(\mathbf{B} \mid \mathbf{x}, \mathbf{w}) P(\mathbf{x} \mid \mathbf{w}) P(\mathbf{w}) \rightarrow P(\mathbf{x}, \mathbf{w} \mid \mathbf{B}) \propto P(\mathbf{B} \mid \mathbf{x}, \mathbf{w}) P(\mathbf{x} \mid \mathbf{w}) P(\mathbf{w}) .
\end{gathered}
$$

where $P(\mathbf{B} \mid \mathbf{x}, \mathbf{w})$ is the likelihood function, $P(\mathbf{x} \mid \mathbf{w})$ is the probability of $\mathbf{x}$ given the uncertainty $\mathbf{w}$ and $P(\mathbf{w})$ is the probability of the existence of the uncertainty. Thus, if the RAOs present a cancellation point at a given pair of wave frequency and direction, the corresponding uncertainty $(w)$ is assumed to have a probability of existence equal to one. Accordingly, see Eq. (4.53), the output of the inference approach will be characterized by sea conditions which present small uncertainties for points of minima and larger ones for the maximums; this is in accordance with the discussion provided in section 3.2.2 $\left(\operatorname{CoV}\left(s_{\zeta \zeta_{n}}\right)=1\right)$.

With regards to the numerical considerations, the resulting matrix $\mathbf{C}$ will have the characteristics described in the following. Furthermore, an illustrative description of the algorithm adopted to estimate $\mathbf{C}$ is provided in Fig. 4.2.

\footnotetext{
${ }^{13}$ An analysis regarding the linearity between the excitations and the RAOs of the platform has been carried out in chapter 5 using coherence functions.
} 


$$
C=\left[\begin{array}{cccccc}
C_{11} & 0 & 0 & \cdots & 0 & 0 \\
0 & C_{22} & 0 & \cdots & 0 & 0 \\
0 & 0 & \ddots & \cdots & 0 & 0 \\
\vdots & \vdots & \vdots & C_{j j} & 0 & 0 \\
0 & 0 & 0 & 0 & \ddots & \vdots \\
0 & 0 & 0 & 0 & \cdots & C_{K M, K M}
\end{array}\right], \quad \text { with } \quad C_{j j} \cdot C_{j j}=\frac{\left|\hat{\mathrm{x}}_{j}-\max (\hat{\mathbf{x}})\right|}{\max (\hat{\mathbf{x}})-\min (\hat{\mathbf{x}})} \sigma^{2} \leq \sigma_{B}^{2}
$$

Consequently, if the alternative prior distribution is used, the posterior distribution is given by:

$$
L(\mathbf{x} \mid \mathbf{B}) \cdot p(\mathbf{x})=K \cdot \exp \left(-\frac{1}{2 \sigma^{2}}\left[\|\mathbf{B}-A \mathbf{x}\|^{2}+\|C \mathbf{x}\|^{2}\right]\right)
$$

As it has been pointed out before, the total size of the maximization problem is still defined by the number of directions $(K)$ and frequencies $(M)$ considered, being the number of variables equal to $K \times M$. Therefore, the solution of the problem is given by the minimization of the following objective function:

$$
J(\mathbf{x})=\|\mathbf{B}-A \mathbf{x}\|^{2}+\|C \mathbf{x}\|^{2}
$$

This minimization is also feasible by means of a conventional quadratic programming algorithm.

Finally, it should be stressed that the alternative approach proposed above is not intended to discredit the advances obtained by other authors or substitute the previous prior distributions ${ }^{14}$, but rather it is an attempt to improve the estimations of the sea conditions based on the observed drawbacks of the current methods.

\footnotetext{
${ }^{14}$ The non-existence of a unique prior distribution that would be appropriate for any inference problem within a given model is established in (DAWID; STONE; ZIDEK, 1973), where the authors showed that the existence of a unique prior is incompatible with a consistent marginalization.
} 


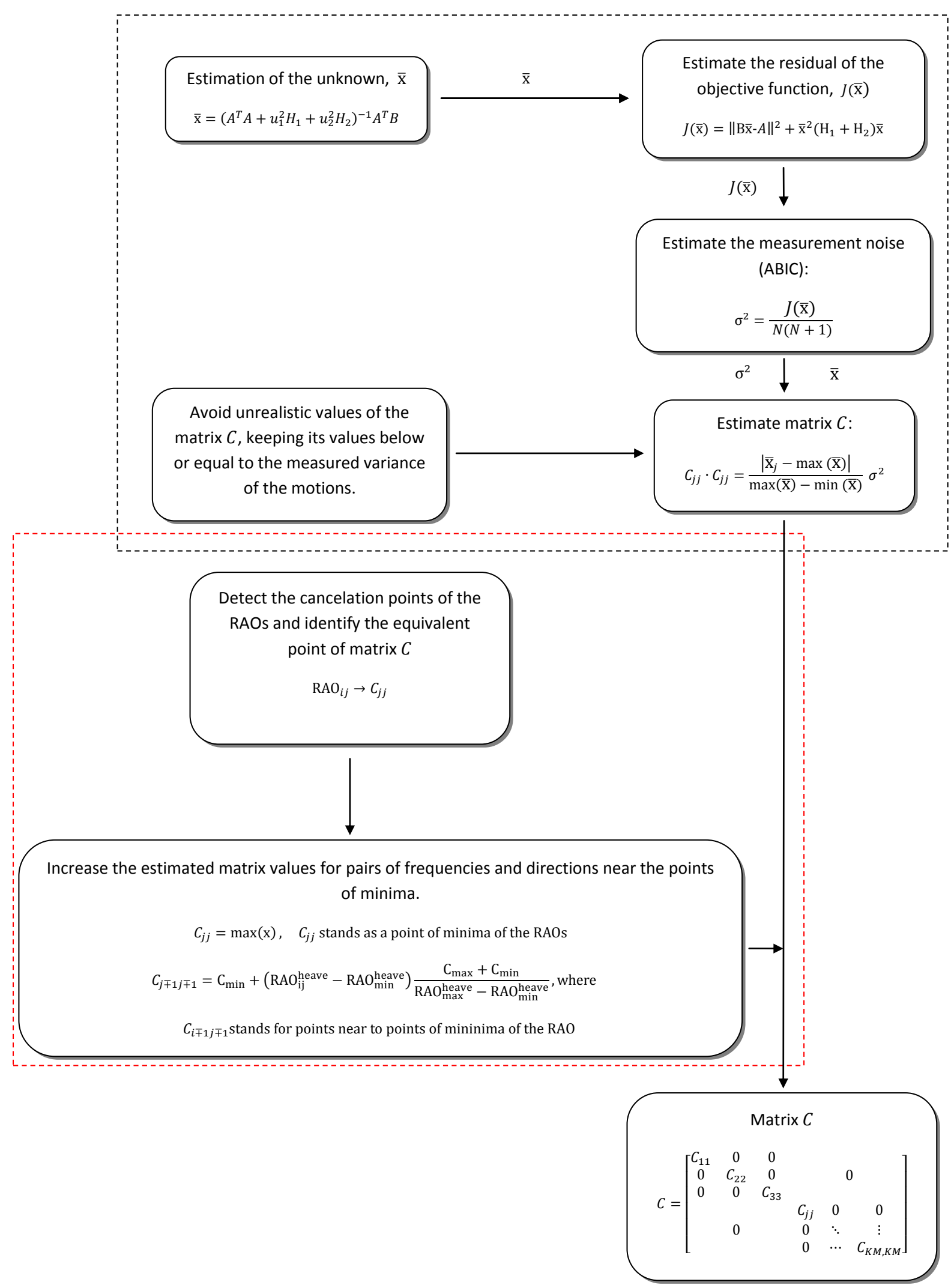

Figure 4.2: Schematic representation of the algorithm adopted to estimate the matrix $\mathbf{C}$. 


\section{Expected improvements of the proposed prior distribution}

At the beginning of this section a description of the main shortcomings of the prior distribution as it is adopted nowadays has been provided. One may realize that the proposed alternative prior distribution is expected to mitigate some of these issues. In fact, the main advantages expected are (a detailed discussion regarding the attainment of these advantages is provided in chapter 5 , based on the experimental measurements):

- This prior distribution does not need previous calibration, in fact it is the result of a self-calibrating procedure that relies on the ABIC information criterion based on the measured motions;

- Since the restraint $\left(\mathbf{H}_{3}\right)$, that avoids unrealistic estimations of the sea wave energy outside of the frequency range of interest, has been replaced by the measurement of the uncertainty in the points of minima, an improvement of the energy estimations in the high frequency range is expected.

- Due to the fact that the approach proposed provides a prior distribution adjusted for each sea condition, it is also expected that the computing time will be reduced.

Although the prior distribution proposed above bring several improvements to the VMB wave inference methodology, it is also characterized by some shortcomings. First, it is important to highlight the fact that the formal description of this new prior distribution is not featured by a direct and clear physical interpretation as it is the case for the prior distribution adopted in the literature. Moreover, in order to avoid estimations of spurious energy peaks as well as poor quality estimations of the sea conditions, there are some assumptions adopted $a d-h o c$, such as the increase of the values of matrix $\mathbf{C}$ close to the cancellation point of the RAOs, thus resulting in a heuristic approach. 


\section{CASE STUDY: THE ÅSGARD-B SEMISUBMERSIBLE PLATFORM}

In this chapter, an assessment of the use of the measured motions of a semisubmersible platform to estimate the sea condition will be made based on model tests performed in a wave basin. For this purpose, the chapter has been organized as follows: First, a brief description of the semisubmersible platform adopted as a case study is provided (section 5.1), followed by a description of the numerical modelling of the hydrodynamic characteristics of the platform (section 5.2). Such description also addresses the importance of a proper damping estimation for predicting the heave resonant motions and the difficulties imposed by the non-linear character of the viscous effects. The experimental setup and the main features of the test campaign are presented in section 5.3. Finally, the chapter includes a detailed comparison of the results obtained when using the prior distribution recommended by (SIMOS et al., 2012) and the alternative prior distribution proposed in this thesis, both previously discussed in chapter 4 .

\subsection{General description}

The study carried out at USP for assessing the use of a semisubmersible platform as a VMB wave sensor has been supported by Equinor (see section 1.1.2), whose main offshore developments are located in the Norwegian sea. Moreover, it was expected that only sea states featured with large wave heights (here the reader is referred to chapter 3, which includes a discussion regarding the semisubmersible hydrodynamic characteristics) could be accurately inferred using this kind of floating platforms. The model employed during the experimental campaign at the wave basin of the TPN-USP was a scaled (1:120) model of the Equinor's Åsgard-B (large) semisubmersible production platform. The adoption of the Åsgard-B platform as a VMB wave sensor was also motivated by the preliminary 
evaluation carried out in (WIJAYA, 2009) (chapter 2 includes a review of the work carried out by Wijaya).

Figure 5.1 shows an aerial photo of the platform as it stands today. The Åsgard-B unit was installed in the year 2000 in the Asgard Field located in the Haltenbank area in the Norwegian Sea, 200km offshore Norway (see Fig. 5.1). The Åsgard development also includes an internal-turret moored FPSO (Åsgard-A unit) for oil and condensate production, along with other facilities, such as the Åsgard-C unit (a storage ship) and several subsea production systems.

The structure of the semisubmersible platform is mainly composed of a rectangular ring pontoon connected to the deck by six box shaped columns ( 4 corner columns and 2 center columns). All the equipment necessary to operate the platform, such as processing facilities, living quarters, utilities to generate power as well as the drilling materials, are located on the top of the deck supporting structure, at elevation of $54 \mathrm{~m}$. The platform is connected to a spread catenary mooring system consisting of 16 lines and a flexible riser system consisting of altogether 37 risers (including simple catenary risers, lazy wave and lazy-S risers) ${ }^{1}$. A general description of the main dimensions of the Asgard-B platform is provided in Tab. 5.1.

The metocean data of the Åsgard (MATHIESEN; NYGAARD, 2013) provides an idea of how harsh the sea conditions might be in the region where the platform is placed. For instance, the (omni-directional) significant wave height in this field is characterized by a mean monthly value that ranges between $3.88 \mathrm{~m}$ (January) and $1.54 \mathrm{~m}$ (July), with a peak periods varying between $2 \mathrm{~s}$ and 23s. Regarding the maximum (omni-directional) monthly wave heights, the average value is equal to $11.41 \mathrm{~m}$, with maximums up to $16.5 \mathrm{~m}$ during the months of December \& January. Moreover, the metocean data shows that the main wind (omni-directional) statistics are featured by a monthly mean wind speed value of $8.67 \mathrm{~m} / \mathrm{s}$ with a standard deviation equal to $1.51 \mathrm{~m} / \mathrm{s}$ and maximum values equal to $33.5 \mathrm{~m} / \mathrm{s}$. Concerning the (omni-directional) data and statistics of the sea currents of this oil field, the measurements carried out attest that the mean velocity usually ranges between $<0.05 \mathrm{~m} / \mathrm{s}$ and $0.6 \mathrm{~m} / \mathrm{s}$.

\footnotetext{
${ }^{1}$ The scaled semisubmersible platform model used during the experimental at the TPN-USP wave basin include neither the elements corresponding to the deck nor the submerged systems (moorings \& risers).
} 

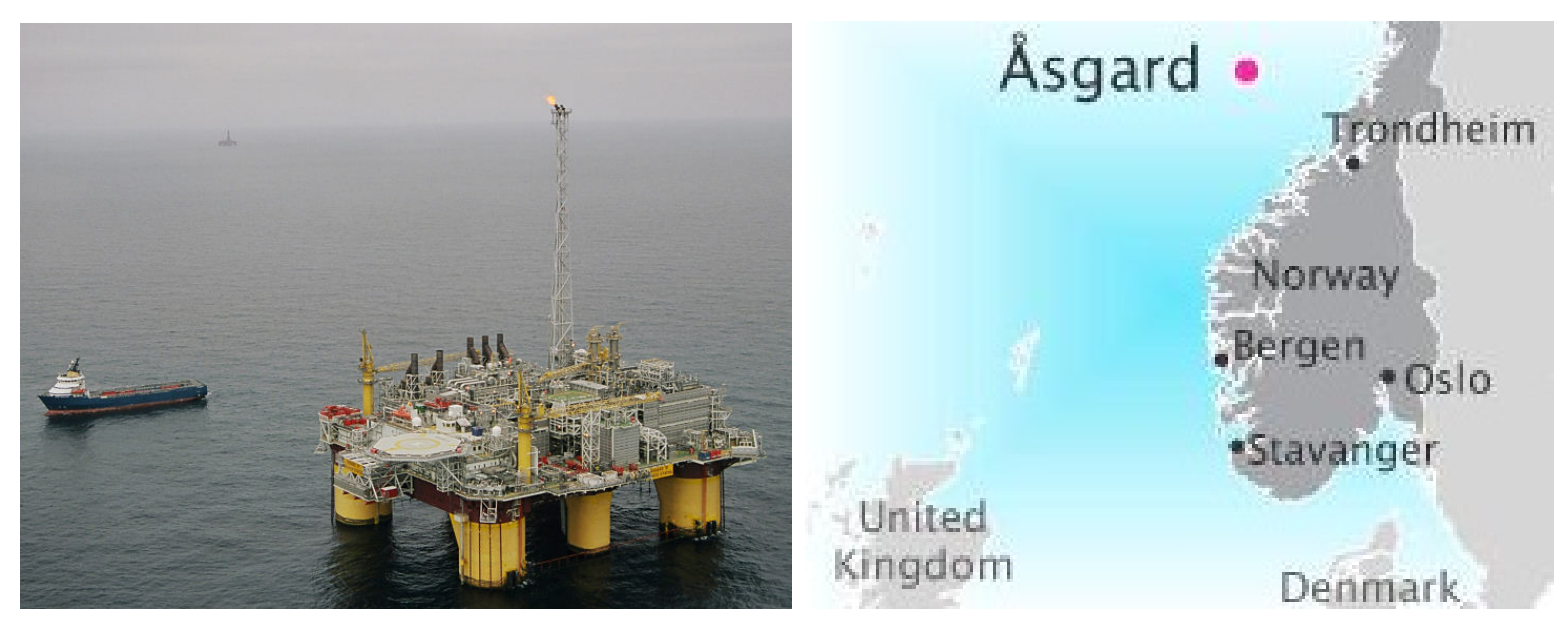

Figure 5.1: Åsgard-B floating gas production platform (left) and a sketch showing the position of the Åsgard field in the Norwegian sea (right), from (STATOIL, 2014).

VMB wave inference will be made based on the three motions in the vertical plane, namely heave, roll and pitch. The adoption of this set of motions as the basis for estimating the sea conditions will be discussed thoroughly in section 5.4.2. At this point, however, an analysis of the dynamic characteristics of these motions is important. The hydrodynamic properties of the semisubmersible unit have been documented by Wijaya (2009). The unit presents a natural period in heave equal to $24 \mathrm{~s}$. The natural periods for roll and pitch are both well above the wave frequency range of interest, being defined as $74 \mathrm{~s}$ and $88 \mathrm{~s}$, respectively.

These sea conditions are characterized by large wave heights and peak periods. Moreover, although the roll and pitch resonances are not expected to be excited, the sea conditions tested present significant amounts of energy close to the natural frequency of the heave motion. Since it is known that the estimation of a linearized viscous damping is an important source of errors concerning the prediction of the resonant responses, one may realize that this platform indeed has good response characteristics for being used as a wave sensor. In fact, the very large natural periods in roll and pitch place their resonances well outside the wave frequency range, thus increasing the reliability of the RAOs of angular motions in this range. Nonetheless, damping corrections in heave motion are still necessary, because this motion presents its resonance inside the range of interest of the current study (focused on long period waves). These corrections have been discussed with detail in section 5.2. 
It is worth to remember that the extreme sea conditions of the Åsgard field present significantly larger peak periods and wave heights that the ones expected, for example, in the Campos Basin (in Brazil). Notwithstanding this, the experimental campaign also included calm/mild sea conditions that may be more representative for other O\&G fields different than the ones encountered in the Norwegian continental shelf.

\section{Scaled model}

The manufacturing process for the model construction took into account the geometrical complexity of the hull and the need to keep the model light weighted (in order to guarantee a proper margin for ballast calibration). Considering these restraints, the 3D printing with nylon sintered material was selected as the best method of construction, except for the deck, which was made of medium density fiberboard (top sides were not modelled, as the tests did not include wind effects). As an illustration a 3D sketch and a photo of the scaled model are presented in Fig. 5.2. The main features of the platform in model scale are also given in Tab. 5.1. The inertial properties of the model (without ballast) were confirmed experimentally by means of pendulum tests (roll and pitch). Inclination tests in still water were made in order to verify the metacentric heights. Finally, regarding the scale of the model $(1: 120)$, it was selected aiming at properly generate all the wave conditions, even the ones characterized by larger wave steepness $\left(\frac{h}{\lambda}\right)$, and avoid to exceed the TPN-USP wave basin limits (although a brief review of the main characteristics of the wave basin is provided in section 5.3, the reader is also referred to (MELLO, 2005) for a detailed description for he TPN-USP wave basin).
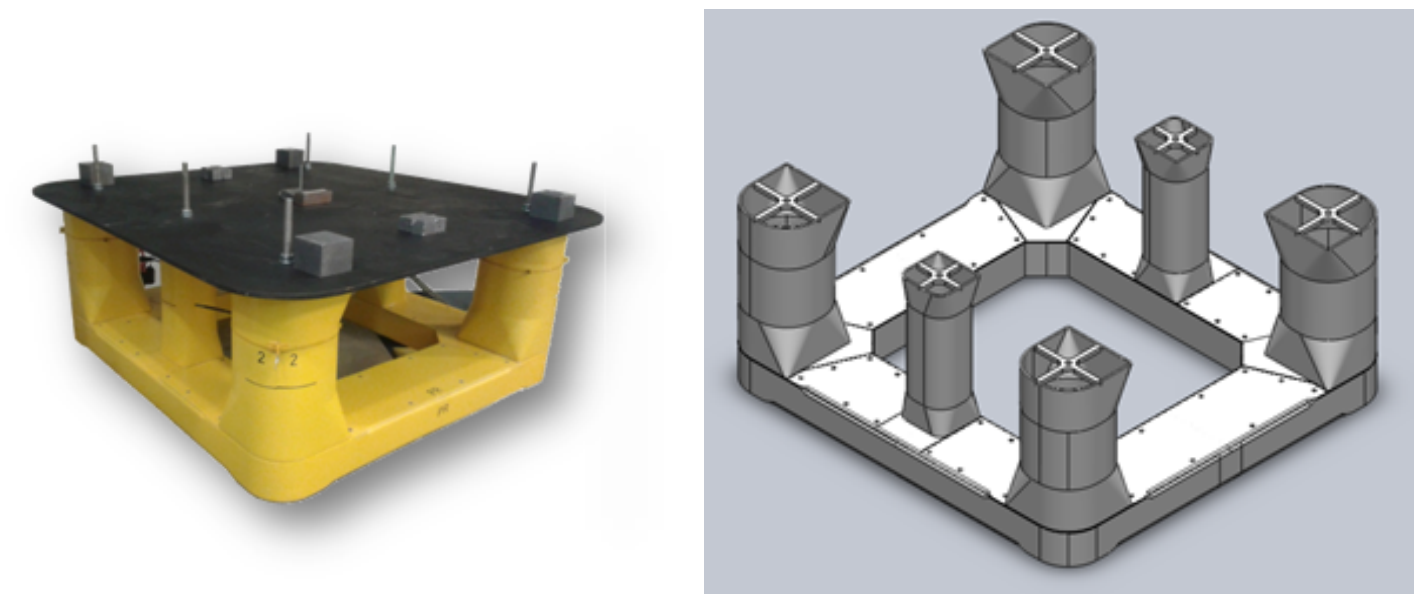

Figure 5.2: The 1:120 scaled semisubmersible platform model. 
Table 5.1: General geometric and mass properties in full-scale and model-scale of the case study.

\begin{tabular}{lcc}
\hline Properties & Full-scale & Small-scale \\
\hline LOA $(\mathrm{m})$ & 102.40 & 0.853 \\
B $(\mathrm{m})$ & 96 & 0.800 \\
D $(\mathrm{m})$ & 45 & 0.375 \\
Draft $(\mathrm{m})$ & 25 & 0.208 \\
Width of pontoons $(\mathrm{m})$ & 19.20 & 0.160 \\
Height of pontoons $(\mathrm{m})$ & 8.96 & 0.074 \\
Diameter, corner columns $(\mathrm{m})$ & 19.20 & 0.160 \\
Diameter, center columns $(\mathrm{m})$ & 12.20 & 0.100 \\
Displacement $(\mathrm{kg})$ & $8.27 \cdot 10^{7}$ & 46.621 \\
Pitch inertia moment $\left(\mathrm{kg} \cdot \mathrm{m}^{2}\right)$ & $1.56 \cdot 10^{11}$ & 2.25 \\
Roll inertia moment $\left(\mathrm{kg} \cdot \mathrm{m}^{2}\right)$ & $1.21 \cdot 10^{11}$ & 2.23 \\
Heave natural period $(\mathrm{s})$ & 24 & 2.19 \\
Pitch natural period $(\mathrm{s})$ & 88 & 8.03 \\
Roll natural period $(\mathrm{s})$ & 74 & 6.75 \\
GM Roll $(\mathrm{m})$ & 3.31 & 0.027 \\
GM Pitch $(\mathrm{m})$ & 3.32 & 0.027 \\
KB Roll $(\mathrm{m})$ & 8.36 & 0.069 \\
KB Pitch $(\mathrm{m})$ & 8.36 & 0.069 \\
BM Roll $(\mathrm{m})$ & 26.86 & 0.223 \\
BM Pitch $(\mathrm{m})$ & 27.07 & 0.225 \\
KG Roll $(\mathrm{m})$ & 31.90 & 0.265 \\
KG Pitch $(\mathrm{m})$ & 31.90 & 0.267 \\
\hline
\end{tabular}

\subsection{Numerical modelling: Motion RAOs and exter- nal damping}

\subsubsection{Introduction}

For this case study, the body's hydrodynamical properties have been obtained with two different approaches: Frequency and time-domain analysis. The former allows to obtain the set of motion RAOs that are used in the wave inference process. Alternatively, time domain simulations were were carried out in order to support the analysis of the nonlinear drag effects, especially those associated with large amplitudes of heave motions near resonance (see chapter 3). A detailed description of the procedures adopted during the numerical modelling is provided next, including the analysis of the main results obtained. 


\subsubsection{Frequency-domain analysis}

RAOs were computed in frequency-domain using the software WAMIT. This software is based on the boundary elements method, also known as panel method, that employs the Green's second identity to derive the boundary integral equations of a distribution of sources and doublets over the surface. Thus, the solution of the problem is computed directly by solving the boundary integral equation system obtained. Moreover, in order to properly model the geometry of the platform the high-order method was adopted, where the potential is represented by means of B-spline functions. In our case, the hull geometry was represented by 52 different patches, which are described by smaller surface elements (panels) with lengths approximately equal to $5 \mathrm{~m}$ (in full scale). Then, resulting in that 4760 panels were used to defined the entire hull of the platform. Figure 5.3 illustrates the geometry considered in this numerical model.
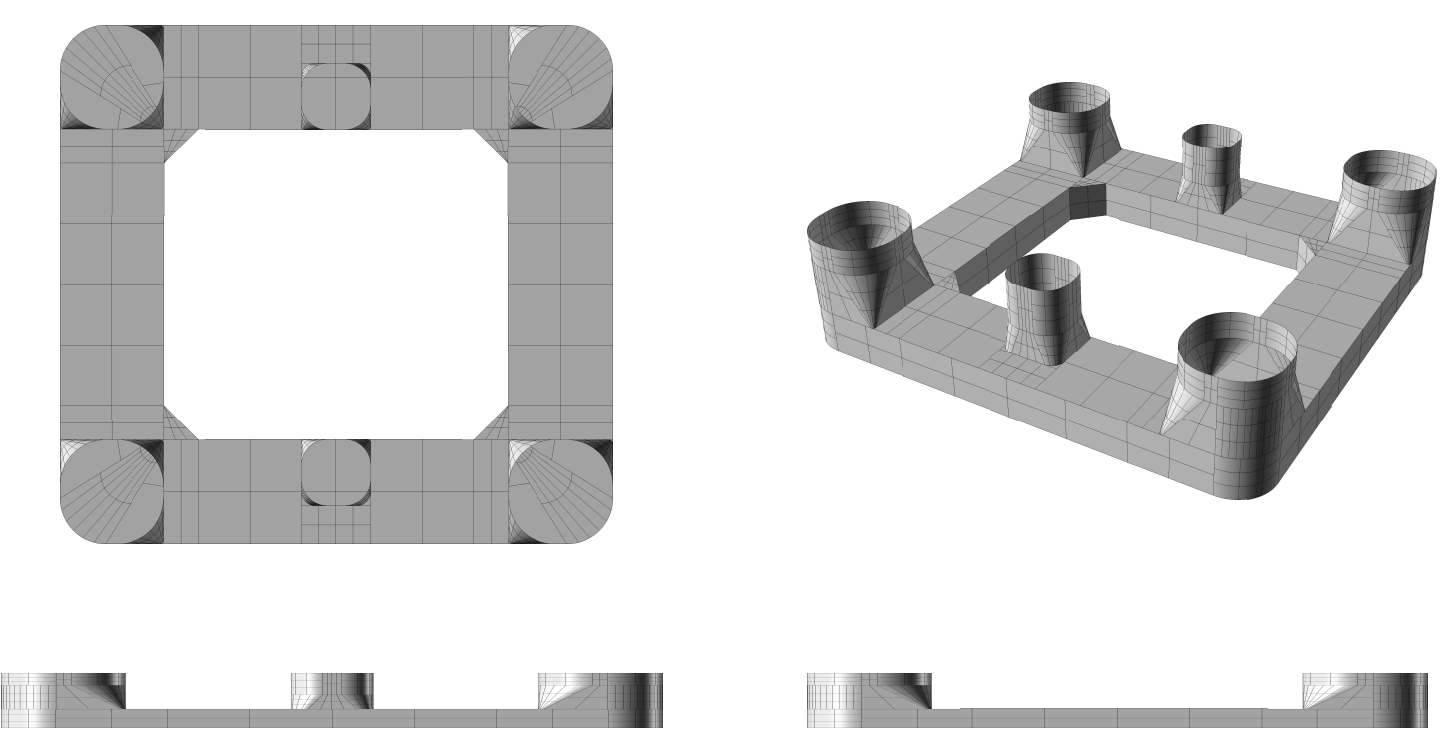

Figure 5.3: Higher order mesh of the semisubmersible platform.

Figure 5.4 shows the RAOs computed in frequency-domain for the heave, roll and pitch motions. Results are presented for five different wave directions, from 180deg. (or bow waves, see Fig. 5.5) to 90deg., beam waves (one should remind that the hull is symmetric in both $\mathrm{x}$ and $\mathrm{y}$ directions). For the sake of illustration, RAOs were computed with external damping equal to $0 \%$ and $5 \%$ (black line), which is a typical value for the external viscous damping of this kind of floating unit and therefore provides a useful reference. The RAOs with $5 \%$ of external damping are only provided for the most unfavorable direction 

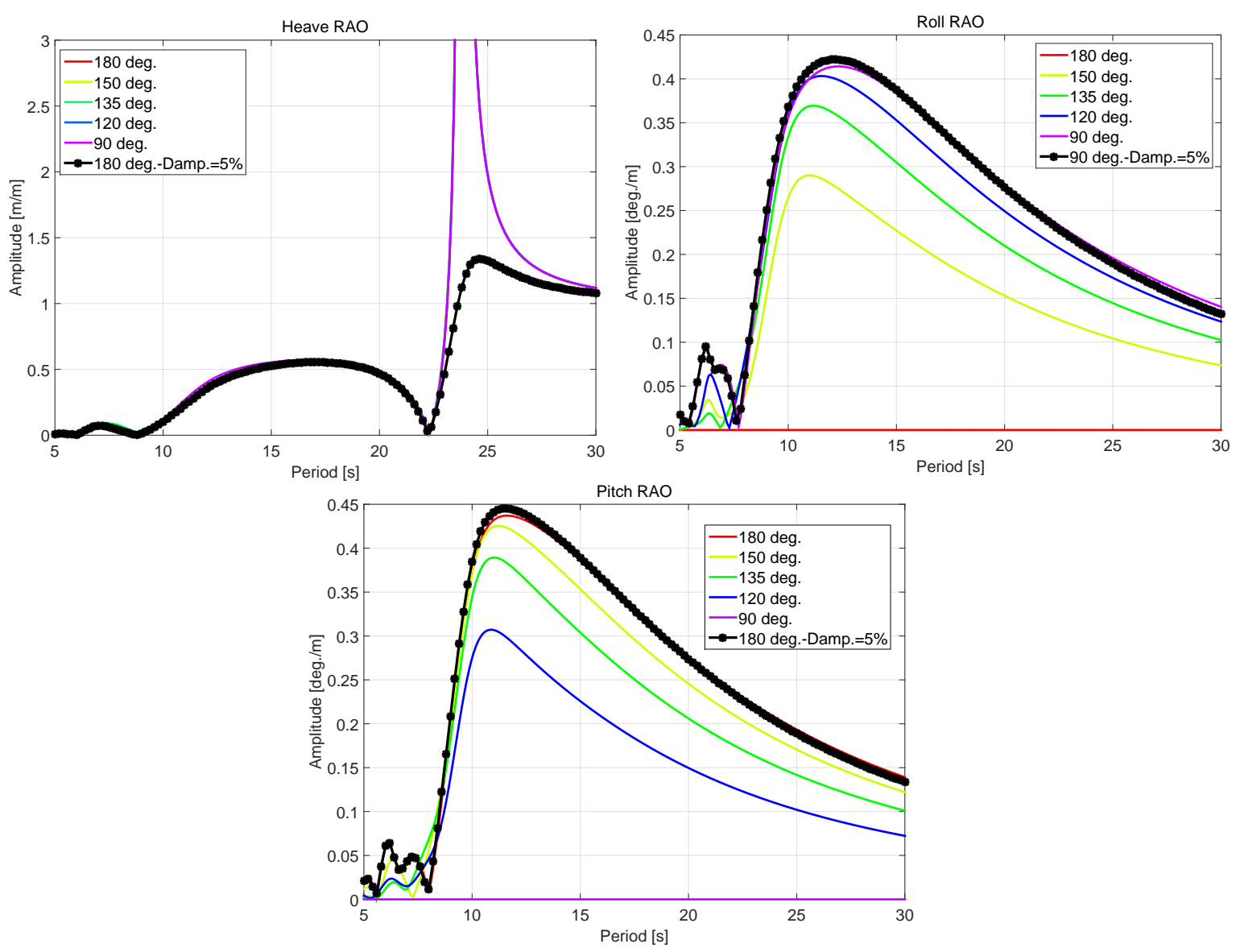

Figure 5.4: RAOs for the motions of heave, roll and pitch.

regarding for each motion, i.e.: 180deg. for heave and pitch and 90deg. for roll motion.

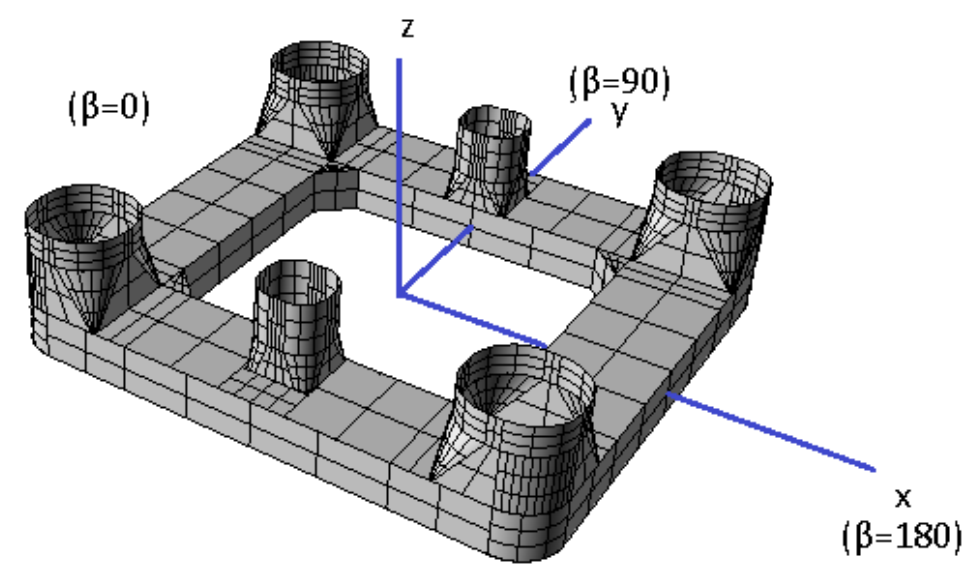

Figure 5.5: Main wave direction relative to the platform.

These RAOs reflect the dynamic characteristics of the platform discussed in section 5.1: The heave motion is the only one with resonance (around 24s, see Tab. 5.1) inside the wave-frequency range (therefore, the external damping effect is only significant 
for the heave RAO); roll and pitch natural periods are around 74 and 88 seconds, respectively, and therefore their motions within the wave frequency range are not supposed to be significantly affected by viscous effects.

In chapter 2 it has been shown that a proper calibration of the external damping is of major importance for an accurate prediction of the responses of the platform close to its resonances. Therefore, the adoption of unreliable external damping coefficients may lead to misleading estimation of the sea conditions that induced the measured motions. Aiming at avoiding inaccurate estimations of the sea state in this case, a dedicated analysis in the domain has been carried out to feature the dynamics of the heave motion of the semisubmersible platform and it is presented in the next section.

\subsubsection{Time-domain analysis}

As aforementioned, the aim of the time-domain analysis is to evaluate the viscous drag forces acting on the pontoons of the platform and their impact on the motions, especially when large amplitudes are involved. The impact of the viscous forces on motion RAOs are widely documented in the literature, e.g. (CLAUSS et al., 2014). In the present case the largest influence of the drag forces are found in the heave motion. A proper evaluation of these effects is necessary, especially because (non-linear) effects may lead to misleading estimations regarding wave conditions with high periods.

\subsubsection{Drag effects arising from the pontoons}

The software used during this stage was the ANSYS-AQWA Hydrodynamic Time Response package. Concerning the frequency domain analysis, the panel model is used to solve the 3-D diffraction/radiation problem and compute the hydrodynamic properties of the structure under study, such as added mass, potential damping and wave excitation forces. The time-domain analysis includes the beam model based on Morison equation (MORISON; JOHNSON; SCHAAF, 1950), see chapter 2, model that is used to estimate drag induced forces (viscous damping force) acting on the platform by means of the characteristics computed in the frequency-domain. Moreover, due to the linear assumptions adopted, the models (in frequency and time-domain) can be combined and the advantages of both models are obtained through this approach. 
In order to perform the analysis in AQWA, the model was approximated by 11830 panels using the low-order model. In addition, the parts of the pontoons between columns were modelled as Morison elements in order to introduce the quadratic drag effects. For instance, the added mass coefficient of these elements, $C_{m}$, was set equal to zero and the drag coefficient, $C_{d}$, was estimated using the data obtained during the decay tests (which are discussed in section 5.3.2).

Figure 5.6 provides an illustration of the heave RAO. Only the heading equal to $180^{\circ}$ is evaluated here, because the heave responses for different headings show only narrow differences if compared to this direction (see Fig. 5.4). The WAMIT frequency-domain analysis (with $0 \%$ and $5 \%$ of external damping) provides a basis of comparison for the timedomain study. It can be seen that both codes provide similar results for the computations in frequency-domain with $5 \%$ external damping.

The figure also presents the results obtained for different regular wave amplitudes using the AQWA time-domain package (indicated by AQWA TD). A first set of results was obtained for a wave steepness of $2 \%$, without considering the Morison elements on the pontoons (blue circles). It can be seen that the RAO results obtained this way agree well with the results obtained from frequency-domain, as expected, due to the fact that non-linear forces have not been included in the analysis. A second set of results was generated including the Morison elements of the pontoon, for wave steepness of $0.64 \%$ and $2 \%$ (magenta rhombus and orange squares, respectively). These results, on the one hand, make the effects of the non-linear drag on the resonant amplification clear (for periods above $24 \mathrm{~s}$ ). On the other hand, they also show that the cancellation point (period of zero heave motion slightly above 22s) expected from the potential flow analysis ceases to exist when the drag forces are considered (the reader is referred to section 3.3.2 and appendix D for a detailed explanation regarding the origin of this cancellation point and its main features). For instance, the resulting excitation force of the heave motion would include a non-linear (extra) drag force component (given by Eq. (3.31)), which leads to a RAO that is dependent, at the same time, of the amplitude of the motion. Accordingly, the value of the heave RAO close to the cancellation is expected to be in accordance with the wave amplitude (i.e. the wave steepness), and therefore reinforcing the relevance of the analysis carried out using different wave steepnesses. 
Figure 5.6 also presents a set of experimental results obtained in regular wave tests (green triangles). All the experimental tests included, for different wave periods, were conducted considering a fixed wave steepness of $2 \%$. One may notice that the experimental results confirm the main trends obtained from the time-domain simulations. As anticipated, these experimental results attest that a proper consideration of the non-linear viscous effects is necessary for a good prediction of the resonant motions.

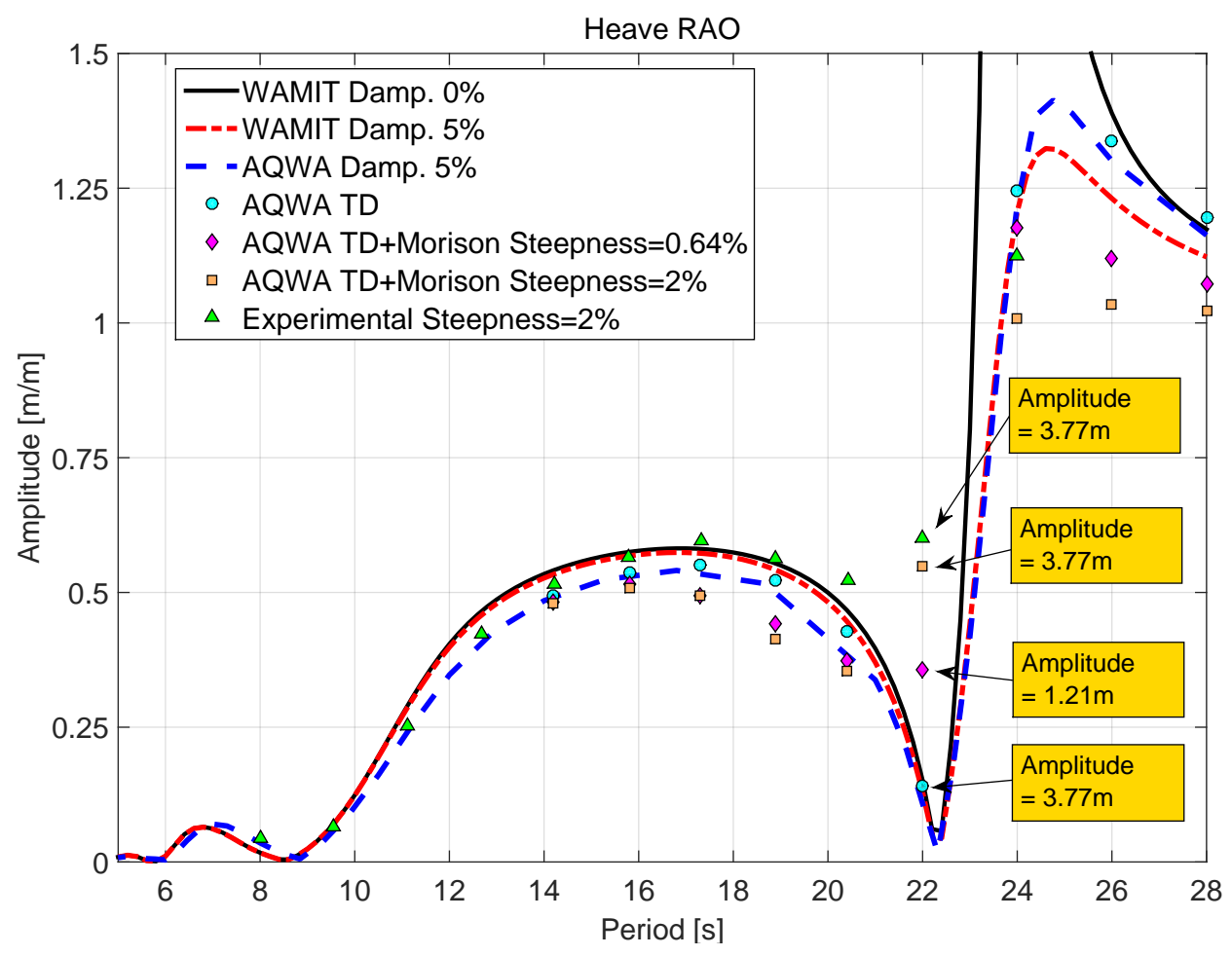

Figure 5.6: RAO heave, for the heading of $\beta=180^{\circ}$.

Finally, it can be highlighted that the main problem, contrary to the results obtained through the linear model, is that for the Asgard-B semisubmersible platform the heave motion RAO values (close to the resonance) will depend on the incident wave amplitude. This non-linear effect must be included in the dynamic model of the heave motion of the floating unit under analysis, topic that has been addressed in section 5.2.4. Regarding fullscale applications, the reader must keep in mind the importance of a dedicated analysis to assess the possible non-linear effects arising from the submerged lines of the system (i.e. moorings and risers) as well as the ones derived from the environmental conditions, such as winds and currents. As a preliminary evaluation, appendix F provides an analysis of the possible effects that the submerged lines may have on the responses of the heave motion close to the resonance. 


\subsubsection{Viscous drag forces and damping linearization}

The reasons why a refined dynamic damping model is needed for the heave motion were already pointed out. From the experimental data obtained during the decay tests (see section 5.3.2), it can be observed that the critical damping factor is strongly correlated with the motion amplitude, as expected. For instance, in section 3.3.2, it has been highlighted that the damping effects in the heave motion are mainly originated by the vertical drag generated by separation effects on the pontoons. Moreover, this is attested by the estimations of the typical $\mathrm{KC}$ numbers of the flow, provided in Fig. 3.4, which show that viscous drag effects on the heave motions become significant in sea conditions characterized by long waves. Therefore, a method to account for this matter and obtain an estimation of the linearized equivalent external damping for different $H_{s}$ is needed.

For this purpose the assumption adopted, and frequently made in the literature, is that the damping is quadratic. In fact, it is common to assume that the steady-state motion with frequency $\omega$ can be written as, see for instance (FALTINSEN, 1993),

$$
\ddot{z}+b_{1} \dot{z}+b_{2} \dot{z}|\dot{z}|+c z=F_{z} \exp \{i \omega t\}
$$

where $b_{1}$ is the linear damping coefficient, $b_{2}$ is the quadratic damping coefficient and $c$ stands for a linear hydrostatic restoring coefficient.

Since the damping arises essentially from the pontoons, an estimation of the external damping can be obtained in accordance for a given value of $H_{s}$, i.e. $\zeta=f\left(H_{s}\right)$. This approximation is possible due to the fact that it is assumed that the platform responses are still linearly related to the amplitudes of incoming waves. Now, using the Morison quadratic drag force formula (MORISON; JOHNSON; SCHAAF, 1950), a correction for the damping factor in the heave motion can be obtained as follows,

$$
\mathrm{d} F_{d}=\frac{1}{2} \rho C_{d} \dot{z}|\dot{z}| \mathrm{d} S \rightarrow F_{d}=b_{2} \dot{z}|\dot{z}|,
$$

being $S$ the cross-sectional area of the pontoon and $C_{d}$ the two-dimensional drag coefficient of the sectional area of the pontoon.

In section 3.3.1 it has been shown that the linearization of the drag force is obtained 
using the well-known method of equivalent energy dissipation (presented, for instance in Faltinsen (1993)). This method results in an equivalent linear drag factor $\left(b_{2}^{\text {Equiv }}\right)$, which stands as a linearization of the drag factor $\left(b_{2}\right)$ and can be written as,

$$
b_{2}^{\text {Equiv }}=\frac{8}{3 \pi} b_{2} \omega z=\frac{8}{3 \pi} b_{2} \omega \operatorname{Re}\left\{A_{z} \exp \left\{i\left(\omega t+\varphi_{z z}\right)\right\}\right\},
$$

where $\omega$ is the frquency and $A_{z}$ represents the (heave) motion amplitude

Then, the resulting equation of motion would be given by,

$$
\ddot{z}+\left(b_{1}+b_{2}^{\text {Equiv }}\right) \dot{z}+c z=F_{z} \exp \{i \omega t\}
$$

Finally, since the platform is characterized by a small potential damping, the damping ratio can be approximated as,

$$
\zeta=\frac{b_{1}+b_{2}^{\text {Equiv }}}{b_{\text {crit }}} \underset{b_{1}<<b_{2}^{\text {Equiv }}}{\longrightarrow} \zeta \approx \frac{b_{2}^{\text {Equiv }}}{b_{\text {crit }}}=\frac{b_{2}^{\text {Equiv }}}{2 \omega_{n}\left(M+a_{33}\right)}=\frac{2}{3 \pi} \rho C_{d} A \frac{l_{\text {pont }}\left(h_{\text {pont }} b_{\text {pont }}\right)}{\left(M+a_{33}\right)} .
$$

where $h_{\text {pont }}$ and $b_{\text {pont }}$ represent the height and the beam of the pontoon, respectively. $l_{\text {pont }}$ is the length of the pontoons in-between columns. $C_{D}$ represents the cross-sectional drag coefficient of the pontoon, $a_{33}$ the heave added mass for the heave natural frequency and $M$ the total mass of the platform.

Therefore, with an appropriate value of $C_{d}$, an estimation of the heave external damping can be made based on a certain representative motion amplitude. In an irregular sea state, however, waves with different frequencies have different amplitudes. Nonetheless, for the purpose of estimating the damping, the most relevant frequency is the resonant frequency of motion. Keeping this in mind, an approximation for the representative amplitude of resonant heave motion can be approximated $\mathrm{as}^{2}$,

$$
A_{z}=\sqrt{2 S_{z z}\left(\omega_{n}\right) \cdot \delta \omega_{n}}
$$

\footnotetext{
${ }^{2}$ It is important to remember that for regular waves a direct correspondence between the measured amplitude, $A_{z}$, and the estimated representative amplitude of motion (given by eq. (5.6)) is expected.
} 
where, once again, $S_{z z}\left(\omega_{n}\right)$ stands as the measured power spectrum of the heave motion at the natural frequency and $\delta \omega_{n}$ is the frequency interval of the spectral analysis close to the natural frequency $\left(\omega_{n}\right)$, or $\left[\omega_{n}-\omega_{(n-1)}, \omega_{(n+1)}+\omega_{n}\right]$. Since the measured power spectrum of the heave motion is known from the records of motions of the platform, this model, thus, allow the estimation of $\zeta$ for each wave estimation event.

In the following section, the damping results obtained with the proposed procedure (referred for now on as variable damping model) are confronted with the damping estimated from the experimental decay tests in heave, showing that indeed a very good fit is achieved.

It should be noticed that since the heave amplitude $A_{z}$ can be obtained directly from the platform motion records, the procedure above can be used for pre-callibrating the nonlinear damping level prior to each wave estimation event. In this way, the implementation is quite simple: All that is needed is a database of RAOs computed for different damping levels, defined within a specified range. At the moment of the wave estimation, the inference algorithm computes the representative heave motion amplitude and selects the most appropriate heave RAO for that particular estimation.

\subsection{Experimental setup and tests description}

\subsubsection{General}

The model tests were carried out in the Numerical Offshore Tank (TPN-USP) facilities, in the USP. The wave basin is a squared $(14.0 \mathrm{~m} \times 14.0 \mathrm{~m} \times 4.1 \mathrm{~m})$ tank able to generate and absorb waves from $0.5 \mathrm{~Hz}$ to $2.0 \mathrm{~Hz}$ (see (MELLO et al., 2013)), by means of a set of 148 flap-type wavemakers. Figure 5.7 shows a sketch of the wave basin and one of the horizontal mooring arrangements adopted during the experimental campaign.

The model was placed in the middle of the basin and its horizontal excursions were restricted by a soft mooring system, above the waterline ${ }^{3}$. As a matter of fact, the objective of the mooring was only to keep the model offsets within the range of optical motion-tracking system. Therefore, the stiffness of the system was kept as low as possible

\footnotetext{
${ }^{3}$ It is worthy to remember that neither mooring lines nor risers were modelled during the experimental campaign.
} 
in order to have little effect on the first order motions of the platform. The overall stiffness in both horizontal directions ( $x$ and $y$ ) was confirmed by means of pull-out tests, obtaining that their values were both equal to $241.05 k N / m$, in full-scale.

In order to guarantee a small influence of the mooring system on the responses of the pitch and roll motions, the lines were attached to the hull of the platform at a height corresponding to the centre of gravity of the model (see Tab. 5.1). Furthermore, the results of the inclination tests were used to ensure that the expected geometric properties (centre of gravity, metacentric height, etc.) did not present significant changes.

The adoption of a mooring system characterized by a low horizontal stiffness resulted in high resonant periods for the motions in the $x-y$ plane, i.e. 113s, 116s and 87s for the surge, sway and yaw motions, respectively. Consequently, the resonances of these motions were not excited by the sea conditions tested during the experimental campaign, which only presented energy in periods ranging between 5 and 33s.

The campaign comprised only one draft (operational) and five wave headings from bow waves to beam waves (in this case: $180^{\circ}, 150^{\circ}, 135^{\circ}, 120^{\circ}$ and $80^{\circ}$ ). Since one end of the tank acted as an actuator (generating the waves) and the other end was used as a wave-absorbing surface for all the sea condition tested, the correct heading conditions were guaranteed by means of changing the (relative) orientation of the model in the tank (always keeping the mooring characteristics unchanged). Finally, it is important to remember that during the tests, the motions of the model were recorded with a Qualisys ${ }^{\circledR}$ optical tracking system.

\subsubsection{Motion decay tests}

Decay tests were performed in still water for motions in the six dofs, in order to determine the natural periods and the damping characteristics of the semisubmersible platform model. For each motion, tests with four different initial amplitudes were carried out, with three repetitions each.

For avoiding spurious excitation of motions other than the ones being tested, a specific set up was used for the decay tests. This set up essentially comprised a set of bars and pulleys with a high resistance wire, used for moving the model and keeping it in the 


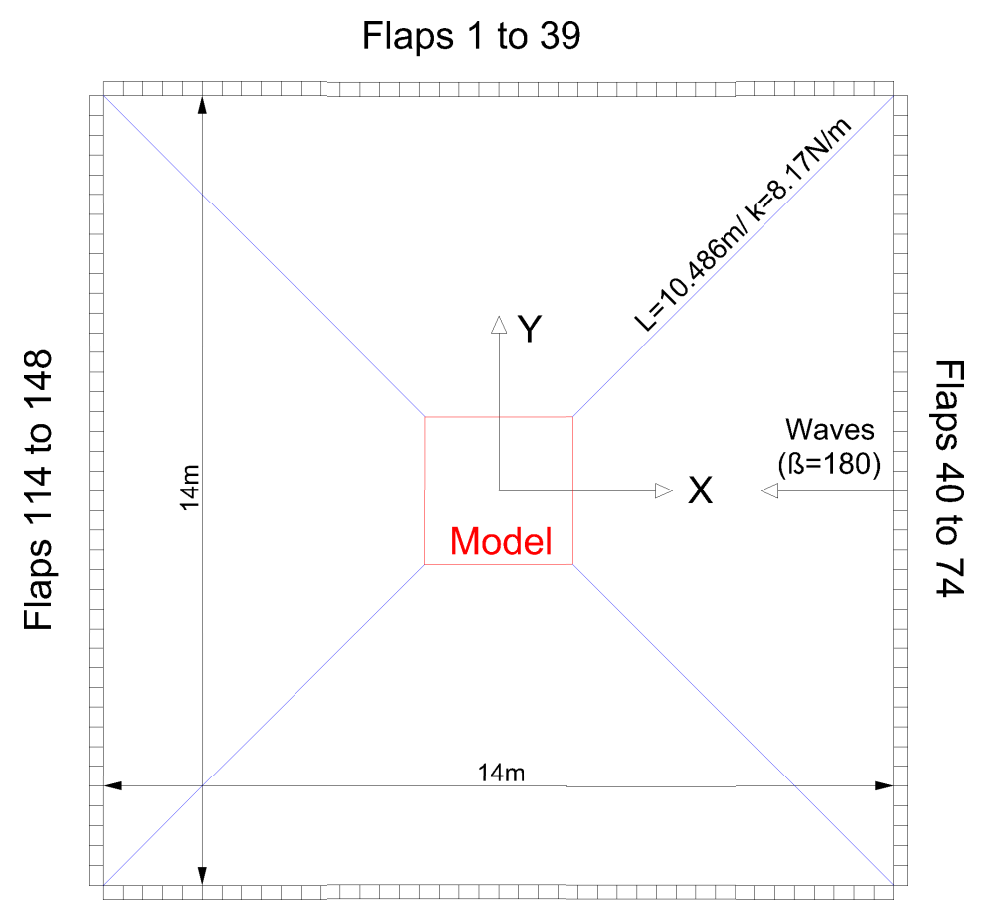

Flaps 75 to 113

Figure 5.7: Sketch of the wave basin and model (not in same scale), including the length and stiffness of the mooring lines. Adapted from Mello (2005).

required initial position for each test. After releasing the model, all the motions were tracked by the Qualisys ${ }^{\circledR}$ optical system. Figure 5.8 provides an illustration of the time series for the decay tests concerning the heave, roll and pitch motions, respectively (in model-scale). From this figure one may realize the similarities between the roll and pitch motions. Furthermore, it shows that the three motions assessed are characterized by a small potential damping, resulting, thus, in time series of the decay tests featured with several cycles.

The method adopted for characterizing the non-linear damping in each test is essentially the one proposed in (FALTINSEN, 1993), which considers a linear and a quadratic damping term (Eq. (5.2)). The former is supposed constant while the later is expressed as a function of the motion amplitude. For illustration purposes, Fig. 5.9 presents the results obtained in tests for four different motions (namely: Heave, roll, pitch and yaw). In this case, however, instead of presenting linear and quadratic damping coefficients, the results are combined and expressed as a percentage of the critical damping ratio for each amplitude $\left(\zeta=\frac{b_{1}+b_{2}}{b_{\text {cirt }}}\right)$.

Variable damping model: A comparison with the results from decay tests 

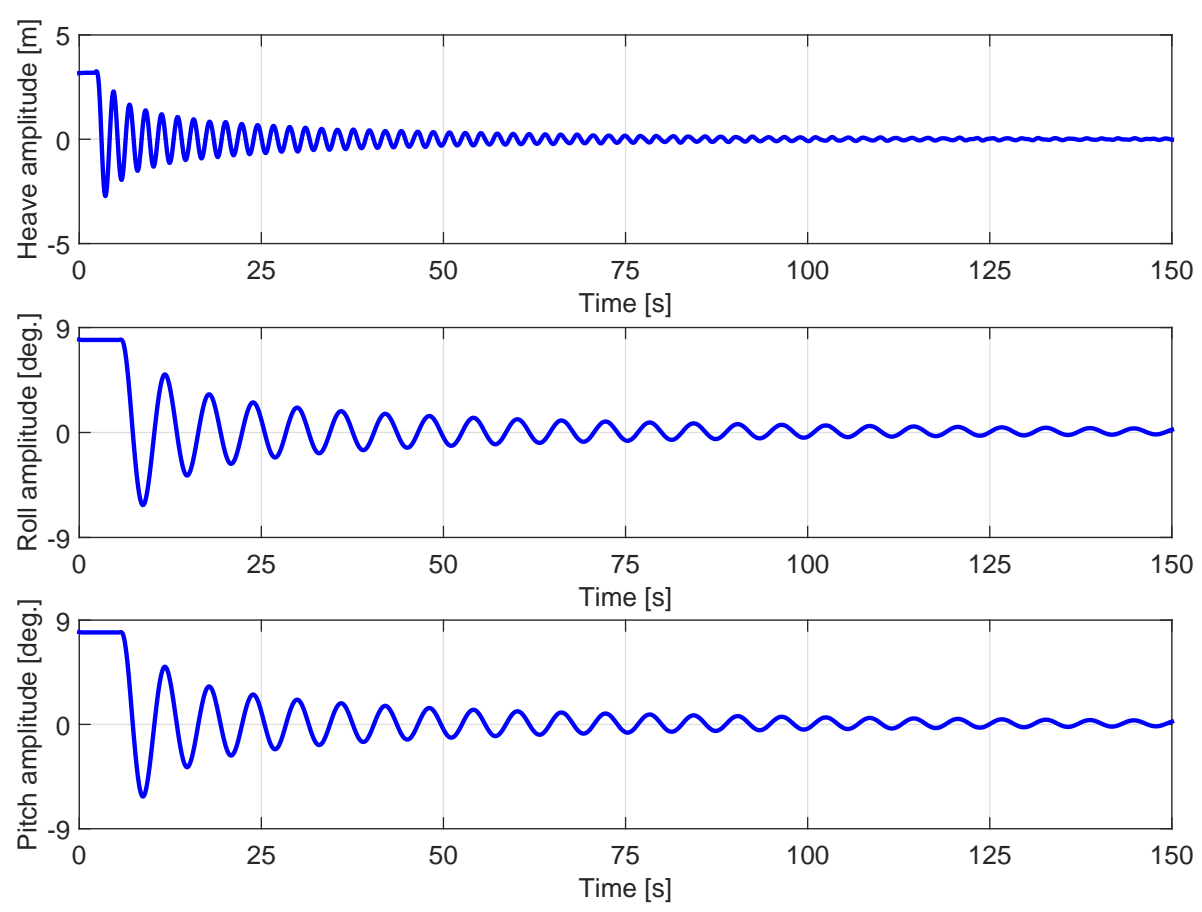

Figure 5.8: Time series of the decay tests, for heave, roll and pitch motions.

Given the geometry of the semisubmersible, with the pontoons placed at relatively large depth, a comparison between damping estimations obtained from tests in waves and in still water (decay tests) is illustrative. For doing this, the method proposed in section 5.2.4 for computing the heave damping from drag estimations was applied. The results were generated considering all the 32 different irregular waves tested in the wave basin, which will be discussed in section 5.3.3 (see Tab. 5.3 for data on the different irregular waves). For each one of them, a representative resonant heave amplitude was obtained according to Eq. (5.6) while the damping ratio was computed with Eq. (5.5). The damping ratios obtained from the irregular wave tests as a function of the resonant heave amplitudes were then compared to the estimations derived from the heave decay tests (see Fig. 5.9). The agreement between the different estimations is shown in Fig. 5.10. One may notice that the damping ratio estimated from the irregular wave tests (black dots) indeed presents narrow differences with the linear fitting (red line) of the data obtained in the heave decay tests performed in calm water (blue triangles).

The good agreement attests that the heave damping is indeed dominated by the pontoon motions and that effect of the wave flow on the damping force is small, even for the largest wave amplitudes that were tested. In this sense, the good fit with the decay 

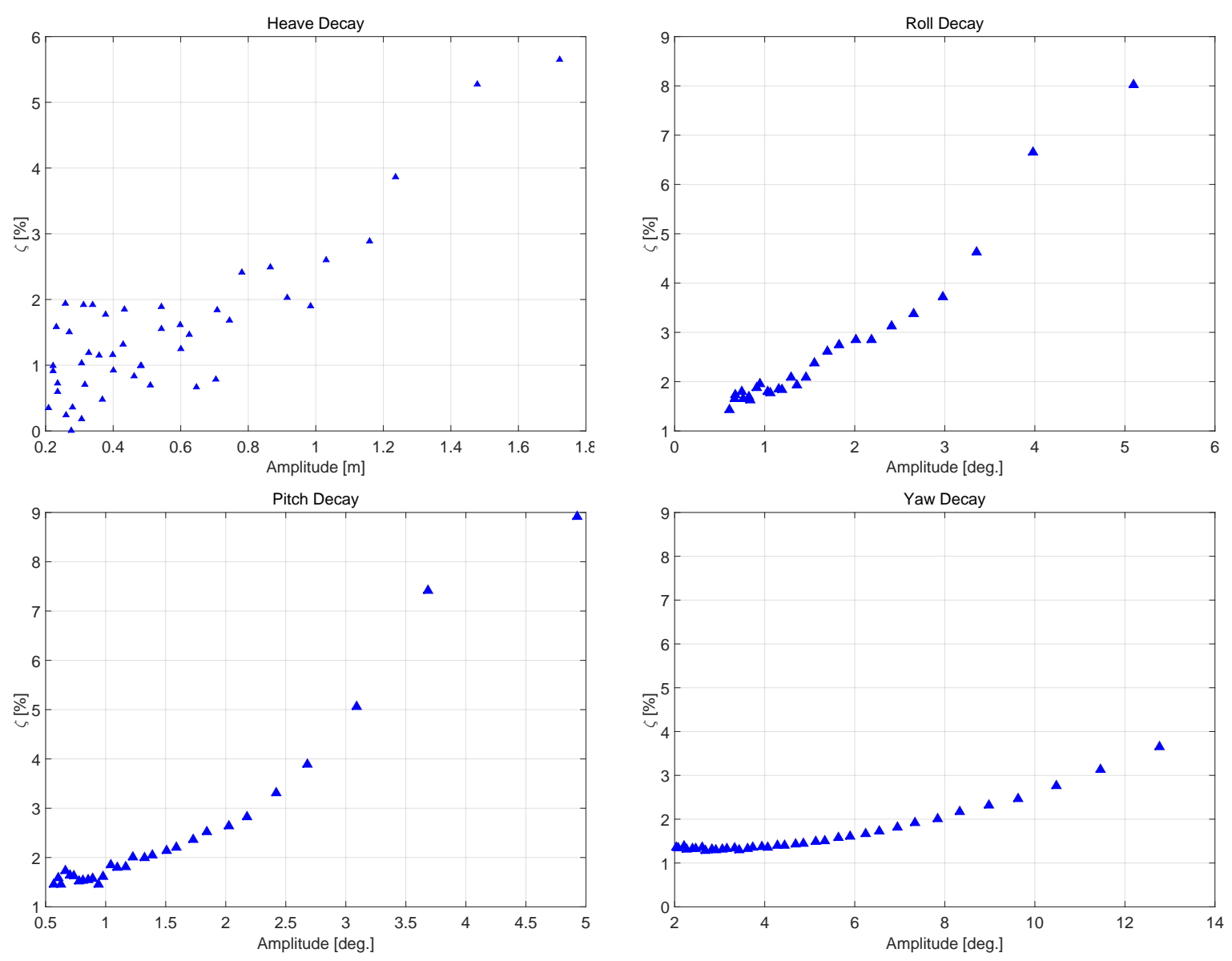

Figure 5.9: Estimated damping ratios for heave, roll, pitch and yaw (from left-to-right then top-to-bottom).

data indicates that the method proposed indeed is adequate for estimating the resonant heave damping of the hull in waves ${ }^{4}$.

\subsubsection{Wave tests}

\section{Transient and regular wave tests}

Transient wave tests (CLAUSS, 1999) were used to verify the accuracy of the linear motion RAOs predicted by the numerical model. For this reason, the wave steepness was kept below $4 \%$ for all the tests. The range of periods for the generation of the transient waves was set from 8 to 24 seconds (values in full-scale), with a constant wave amplitude equal to $1.68 \mathrm{~m}$. Tests had a duration equivalent to 240 seconds in full-scale, guaranteeing

\footnotetext{
${ }^{4}$ It is important to keep in mind that the effects of mooring lines as well as risers are not included in this assessment. The reader is referred to appendix F for a preliminary evaluation of the possible dynamic effects resulting from the submerged lines.
} 


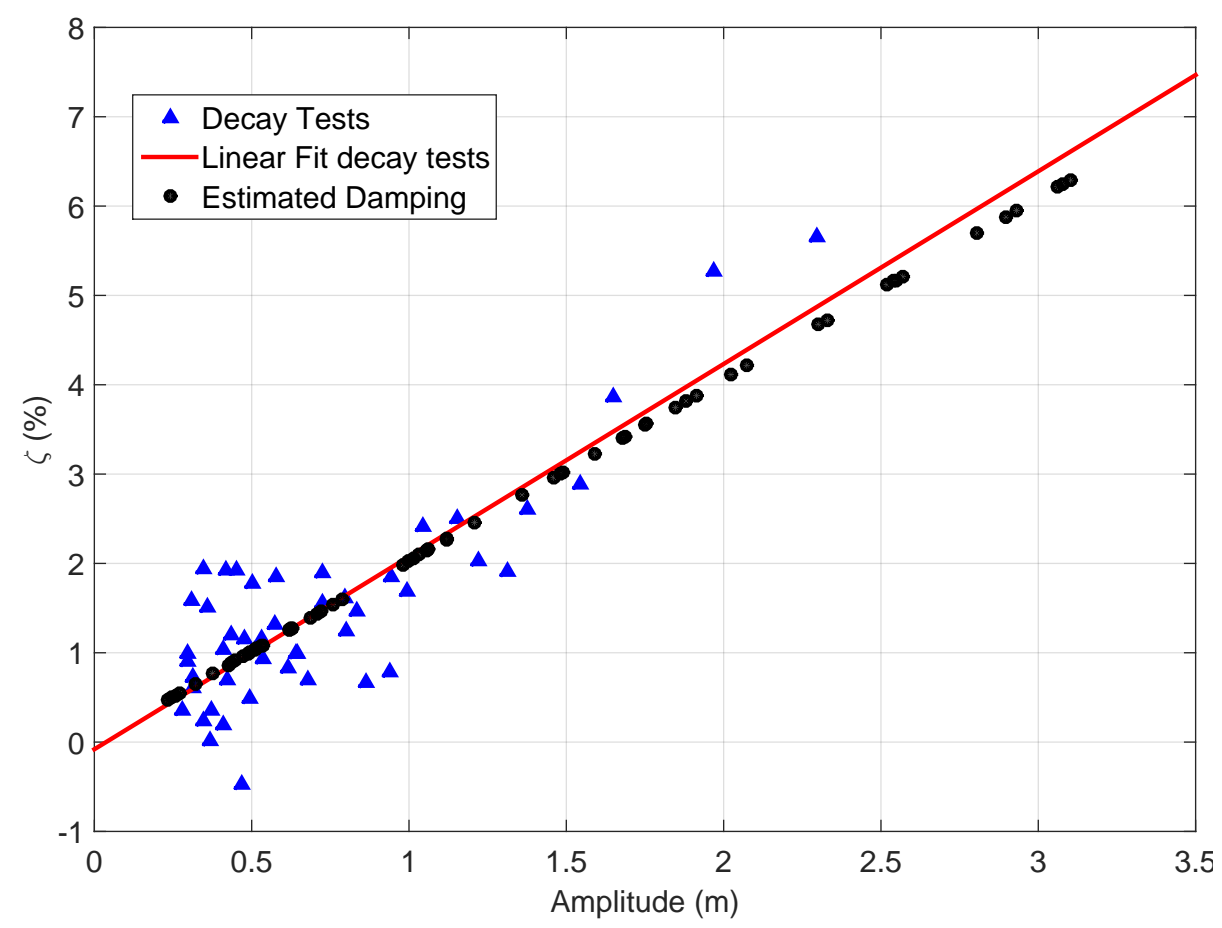

Figure 5.10: Linearized heave damping factor (black points) estimated from wave tests confronted with the experimental results of decay test presented in figure 5.9 (blue triangles) which are linearly fitted with the red line.

at least 10 cycles (ITTC, 2014) of the heave motion in the resonant frequency.

In addition, a set of 33 tests in regular waves was carried out for a better evaluation of the non-linear effects on the RAOs. Again, the wave period ranged from 8 to 24 seconds, but different wave amplitudes were tested corresponding to wave steepnesses of $2 \%, 4 \%$ and $6 \%$.

Figure 5.11 illustrates the level of agreement obtained between the motion RAOs derived from the wave tests (both for transient and regular waves) and those derived numerically. Results correspond to a wave heading of $135 \mathrm{deg}$. and comprise only the three motions that were used for the purpose of wave inference (heave, roll, pitch). Due to the technical limitations of the wave basin, the estimations of the RAOs obtained by means of the regular waves with $4 \%$ and $6 \%$ steepnesses arrive up to 22s. Nonetheless, it can be seen that the agreement for the angular motions is good for the whole range of frequencies. This was indeed expected because neither roll or pitch resonate in the wave frequency range. For the heave motion, on the other hand, the agreement is good for wave periods up to 20 seconds. As anticipated in chapter 3 and in section 5.2.3, discrepancies 
rise for larger periods and are associated to viscous effects within the resonant range (see Fig. 3.4). In this case, the numerical heave RAOs were computed with $\zeta=5 \%$ as well as with $\zeta=3.81 \%$ and $\zeta=6.89 \%$. The two last damping values (3.81\% and $6.89 \%)$ were computed through the procedure proposed in section 5.2.4 and correspond to the amplitude deduced from the transient wave and the regular wave with steepness equal to $2 \%$, respectively. Since the effects that the damping has on the numerical roll and pitch RAOs in the period range of interest are negligible, only the roll and pitch RAOs computed with $\zeta=5 \%$ have been included.
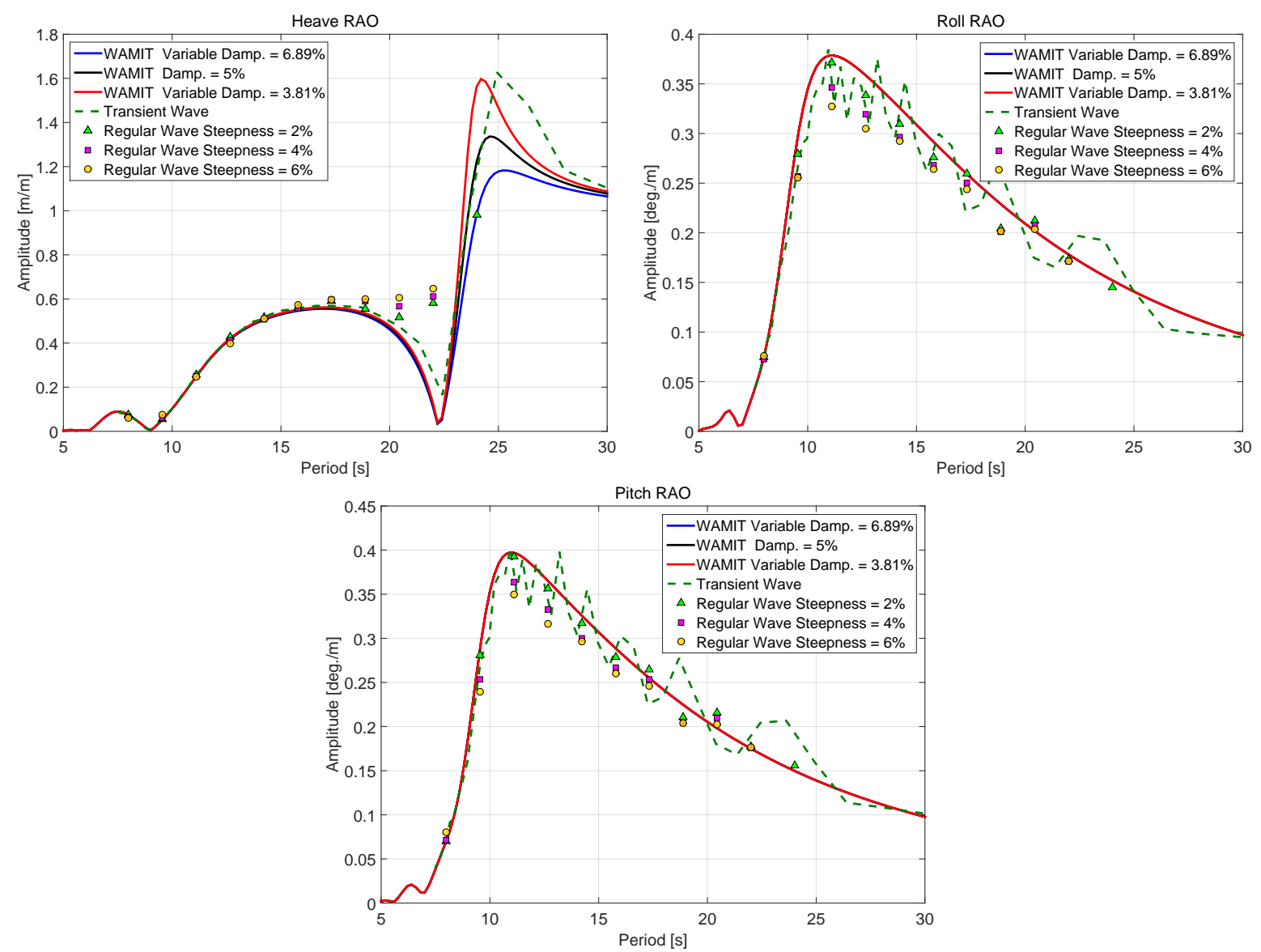

Figure 5.11: Comparison of the (heave, roll \& pitch) RAOs estimated numerically (black line) with the experimental results, both transient (red dashed line) and regular waves with $2 \%$ of steepness (green triangles), for a heading of $135^{\circ}$.

Regarding the use of the heave motion for wave inference, these results show that the possible discrepancies close to the resonance can be mitigated with the use of a proper damping model, which provides a better adjustment of the resonant amplification for wave periods around the natural one. Another source of error, namely the one related to the motions observed close to the numerical cancellation point, is, however, more difficult to 
mitigate.

\section{Irregular wave tests}

The wave conditions for the of irregular waves (sea states) were selected from the wave statistics of the Åsgard field documented by Mathiesen and Nygaard (2013). Along with the brief description of the main characteristics of this field provided in section 5.1, Tab. 5.2 provides a general picture regarding the annual probability of exceedance of the (omni-directional) extreme wave conditions (significant wave height and peak period). Furthermore, as previously pointed out in section 3.2.2, the JONSWAP and Torsethaugen wave spectra stand as the most representative wave spectrum models for the sea conditions observed in the Asgard field.

Table 5.2: Annual probability of the input parameter used for some of the sea tests conditions.

\begin{tabular}{ccc} 
Annual probability of exceedance & $H_{s}(m)$ & $T_{p}(s)$ \\
\hline 0.63 & 11.7 & 15.7 \\
$10^{-1}$ & 14.1 & 16.9 \\
$10^{-2}$ & 16.4 & 18.1 \\
$10^{-4}$ & 20.8 & 20.3 \\
\hline
\end{tabular}

The experimental campaign comprised a set of 32 sea conditions with their corresponding peak periods $\left(T_{p}\right)$ and significant wave heights $\left(H_{s}\right)$, ranging from mild waves with high probability of occurrence to extreme 100yr-return events. It should be highlighted that for the experimental campaign carried out with the semisubmersible platform only long crested sea conditions were generated due to technical limitations of the wave basin. Following the recommendation proposed in (TORSETHAUGEN; HAVER, 2004), some of the sea conditions were generated using a double peak (in frequency-domain) wave spectrum, more specifically the Torsethaugen wave spectrum.

The sea conditions generated in the wave basin by means of this spectrum were selected based on the approach proposed by Torsethaugen and Haver (2004). This methodology is based on the estimations of a peak period threshold, which is referred to by the authors as limiting spectral peak period and defined by the relation $T_{p_{f}}=6.6 H_{s}^{1 / 3}$. Thus, if the expected peak period of the sea $\left(T_{p}\right)$ was located within a band given by $T_{p_{f}} \pm 2$ seconds, the JONSWAP standard model was adopted to generate the spectrum and otherwise, the spectrum was generated using the Torsethaugen spectrum. Figure 3.1 
provides a comparison between the Torsethaugen spectrum and an equivalent JONSWAP spectrum for the same significant wave height and peak period.

Table 5.3: Input parameters used for the sea tests conditions. The wave direction in the tank was considered as $180^{\circ}$ for all the tests conditions.

\begin{tabular}{|c|c|c|c|c|}
\hline Wave ID & Sea Spectrum & $H_{s}(m)$ & $T_{p}(s)$ & Wave Steepness (\%) \\
\hline STA-IRR-01 & JONSWAP & 3.5 & 9.5 & 2.48 \\
\hline STA-IRR-02 & JONSWAP & 3.5 & 11.5 & 1.70 \\
\hline STA-IRR-03 & TORSETHAUGEN & 3.5 & 13.5 & 1.23 \\
\hline STA-IRR-04 & TORSETHAUGEN & 3.5 & 15.5 & 0.93 \\
\hline STA-IRR-05 & TORSETHAUGEN & 3.5 & 18.5 & 0.65 \\
\hline STA-IRR-06 & JONSWAP & 4.5 & 10.5 & 2.61 \\
\hline STA-IRR-07 & JONSWAP & 4.5 & 12.5 & 1.84 \\
\hline STA-IRR-08 & TORSETHAUGEN & 4.5 & 14.5 & 1.37 \\
\hline STA-IRR-09 & TORSETHAUGEN & 5.5 & 9.5 & 3.90 \\
\hline STA-IRR-10 & JONSWAP & 5.5 & 11.5 & 2.66 \\
\hline STA-IRR-11 & JONSWAP & 5.5 & 13.5 & 1.93 \\
\hline STA-IRR-12 & TORSETHAUGEN & 5.5 & 18.5 & 1.03 \\
\hline STA-IRR-13 & JONSWAP & 6.5 & 12.5 & 2.66 \\
\hline STA-IRR-14 & TORSETHAUGEN & 6.5 & 16.5 & 1.53 \\
\hline STA-IRR-15 & JONSWAP & 7.5 & 11.5 & 3.63 \\
\hline STA-IRR-16 & JONSWAP & 7.5 & 13.5 & 2.64 \\
\hline STA-IRR-17 & TORSETHAUGEN & 7.5 & 18.5 & 1.40 \\
\hline STA-IRR-18 & JONSWAP & 8.5 & 12.5 & 3.48 \\
\hline STA-IRR-19 & JONSWAP & 9.5 & 12.5 & 3.89 \\
\hline STA-IRR-20 & JONSWAP & 9.5 & 14.5 & 2.89 \\
\hline STA-IRR-21 & TORSETHAUGEN & 9.5 & 16.5 & 2.23 \\
\hline STA-IRR-22 & TORSETHAUGEN & 9.5 & 18.5 & 1.78 \\
\hline STA-IRR-23 & JONSWAP & 10.5 & 14.5 & 3.20 \\
\hline STA-IRR-24 & JONSWAP & 11.7 & 14 & 3.82 \\
\hline STA-IRR-25 & JONSWAP & 11.7 & 15.7 & 3.04 \\
\hline STA-IRR-26 & TORSETHAUGEN & 11.7 & 18.1 & 2.29 \\
\hline STA-IRR-27 & JONSWAP & 14.1 & 15.7 & 3.66 \\
\hline STA-IRR-28 & JONSWAP & 14.1 & 16.9 & 3.16 \\
\hline STA-IRR-29 & TORSETHAUGEN & 14.1 & 19.3 & 2.42 \\
\hline STA-IRR-30 & JONSWAP & 16.4 & 16.9 & 3.68 \\
\hline STA-IRR-31 & JONSWAP & 16.4 & 18.1 & 3.21 \\
\hline STA-IRR-32 & TORSETHAUGEN & 16.4 & 19.5 & 2.76 \\
\hline
\end{tabular}

Table 5.3 shows the input parameters used for all the sea tests conditions. During the experimental campaign the time duration of each test was equivalent to three hours in full-scale (16.4min in model-scale), which were split in two intervals of 1.5 hours each ${ }^{5}$. Moreover, the wave spectrum was generated by means of summing a sufficiently large

\footnotetext{
${ }^{5}$ This condition was imposed due to technical limitations of the wave generation-absorbtion system of the wave basin.
} 
number of sinusoidal wave components $(N=2000)$, whose amplitudes were derived from the theoretical spectra. Also, the phase seed used to generate the randomness of the wave record was different for each $1.5 \mathrm{~h}$ half test, providing two different realizations of the same sea condition.

In order to provide a general idea of the results obtained Fig. 5.12 has been included, which presents the water surface elevation for the first realization of sea condition $S T A-I R R$-29. In this case $H_{\max }$ corresponds to $23.4 \mathrm{~m}$ and over the whole experimental campaign the maximum wave height recorded was equal to $26.6 \mathrm{~m}$.

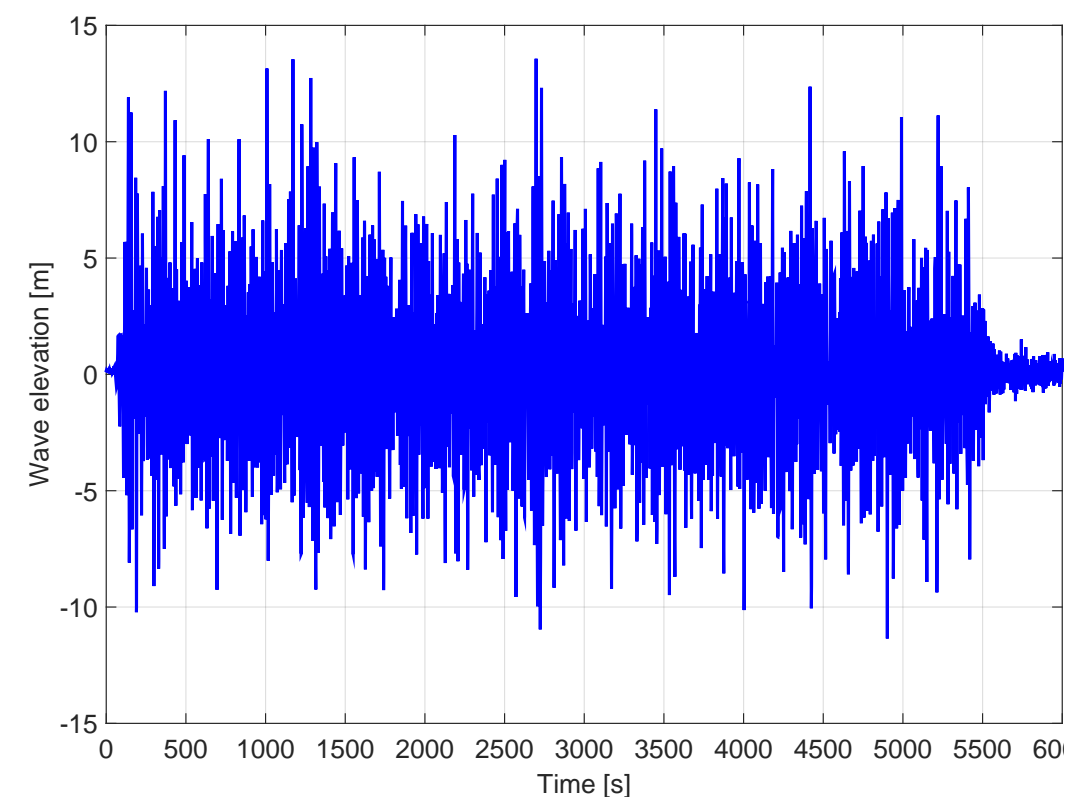

Figure 5.12: Time series of surface elevation of the first realization of sea condition STAIRR-29, with $H_{s}=14.1 \mathrm{~m}$ and $T_{p}=19.3 \mathrm{~s}$.

As a complement to this figure and aiming at providing a general idea of the waves generated during the experimental campaign Fig. 5.13 shows the model-scale of the semisubmersible platform during an irregular wave test. From this picture the reader may realize the severity that characterized the wave generated during the experimental campaign.

Furthermore, as a complementary material to the wave elevation, Fig. 5.14 shows the measured heave, roll and pitch motions induced by the time series provided in Fig. 5.12.

Finally, Fig. 5.15 provides an illustration of the differences between the realization of the same sea condition, as a consequence of using different phase seeds. The variances of the energy of the spectrum observed in this figure are in accordance with the theory 


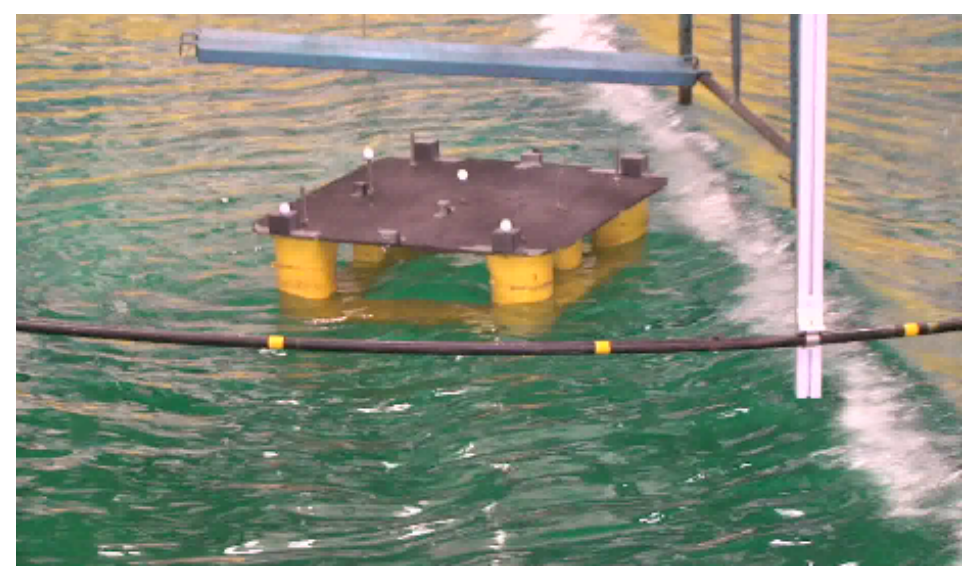

Figure 5.13: Picture of the model-scale of the semisubmersible paltform during one irregular wave sea condition in the TPN-USP.
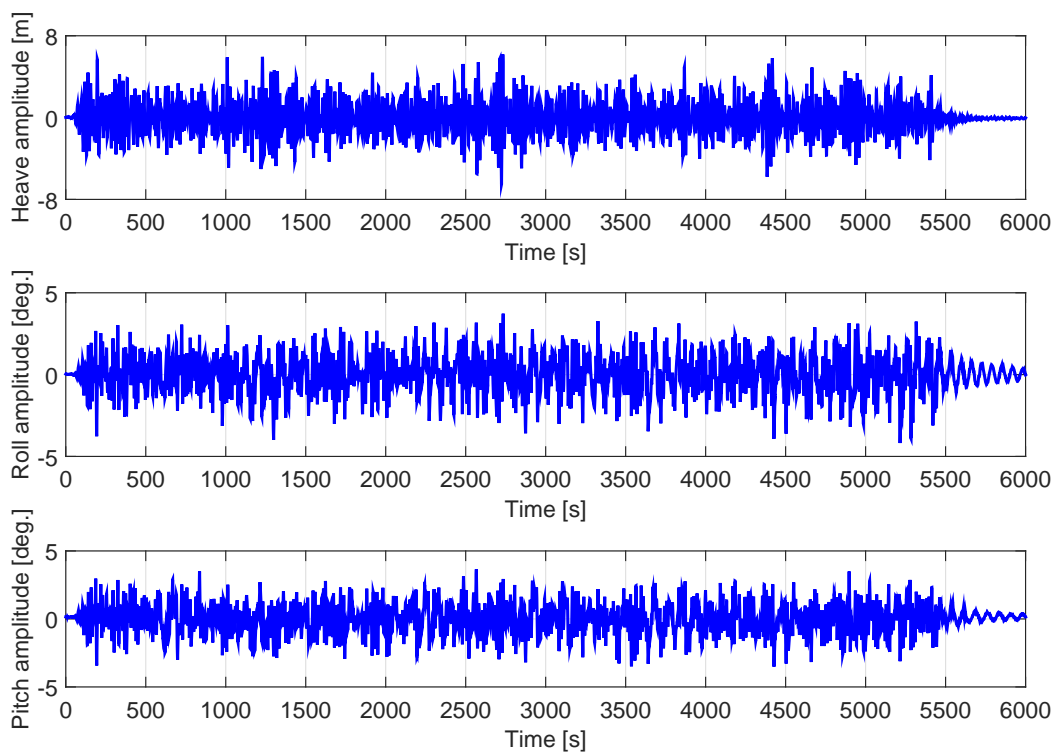

Figure 5.14: Recorded heave, roll and pitch motion time series for sea condition STAIRR-29.

provided in chapter 3.2 .

\section{Wave calibration}

Wave calibration tests were performed in the absence of the semisubmersible model and the evaluation of the wave spectrum within the test zone was performed by means of an array of three wave probes. The quality of the waves generated in the basin was checked considering the main statistical parameters. In most of the cases, the values of $T_{p}$ and $H_{s}$ were close to the requested values in the first wave execution. In this calibration procedure, the maximum frequency of the spectrum computed was $2 \mathrm{~Hz}$, corresponding 

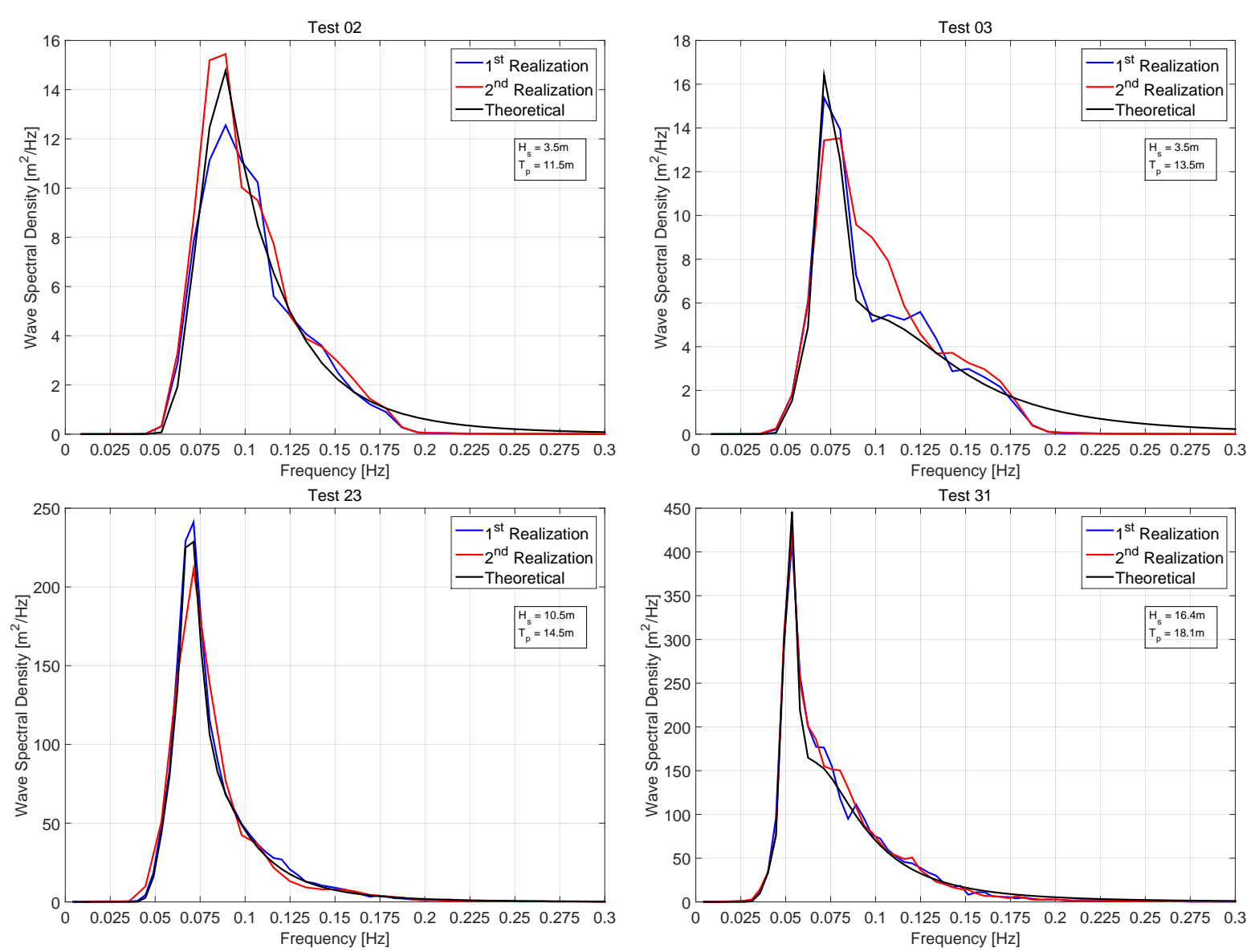

Figure 5.15: Variability that the sea spectrum generated in the wave basin presents, for each realization, and sea conditions corresponding to tests: $2,3,23,31$.

to 5.5 seconds in full-scale. The procedure has guaranteed that errors in the values of $T_{p}$ and $H_{s}$ were lower than $5 \%$ for all tests.

\subsubsection{Wave spectra estimation from wave probes}

As pointed out in section 5.3.3, wave calibration tests were performed in the absence of the semisubmersible model by means of an array of three wave probes. Figure 5.15 showed some examples of the sea spectrum measured. It included sea states generated using a JONSWAP and Torsethaugen sea spectra, as well as different sea conditions. From this figure one may realize that all the different sea conditions were generated with reasonable accuracy. 


\section{Experimental measurements of the directional wave spectrum}

In order to provide a reference for evaluating the accuracy of the seas estimated using the VMB wave inference algorithm, an array of eight wave probes has been used to measure the directional wave spectrum in the wave basin. Once again, the measurements were made in the absence of the semisubmersible model. The procedure adopted to measure directional waves follows the one proposed by Nwogu (1989), applying the Maximum Entropy Method (MEM). A good explanation of the procedure adopted for estimating the spectrum from the wave records can be found in Tannuri et al. (2007). The disposition of the wave probes is similar to the one proposed in Stansberg (1998), with six capacitive wave probes placed in the vertices of a regular hexagon with a circumradius equal to $0.4 \mathrm{~m}$, plus two central ones. Figure 5.16 provides a view of the wave probes arrangement installed in the basin.

For illustrative purposes, Fig. 5.17 presents the results obtained with MEM method for the irregular waves generated in four different tests, which were selected among the 32 sea conditions in order to provide a representative sample of the whole range of periods that was tested. In these polar plots, the wave energy in each wave frequency (radial coordinate in $\mathrm{rad} / \mathrm{s}$, with values given in full-scale) is depicted for all wave directions (as a convention, the wave direction in the tank was considered as 180deg.). In addition, the main statistical parameters for each wave condition are presented at the top of each figure. It should be stressed that, instead of the peak period, the mean central period $T_{1}$ is used for evaluating the results (as diescussed previously in section 3.2).

All the waves generated in the basin were long-crested, as pointed out in section 5.3.3, meaning that no directional energy spreading was considered in the wave generation. For this reason, one may realize that the wave spectra measured with the MEM method have energy concentrated in a very narrow range around the mean direction (the directional resolution considered in the spectral analysis corresponded to $\Delta \theta=5$ degrees, the same that has been employed for the VMB wave inference, whose results will be presented in section 5.4). 


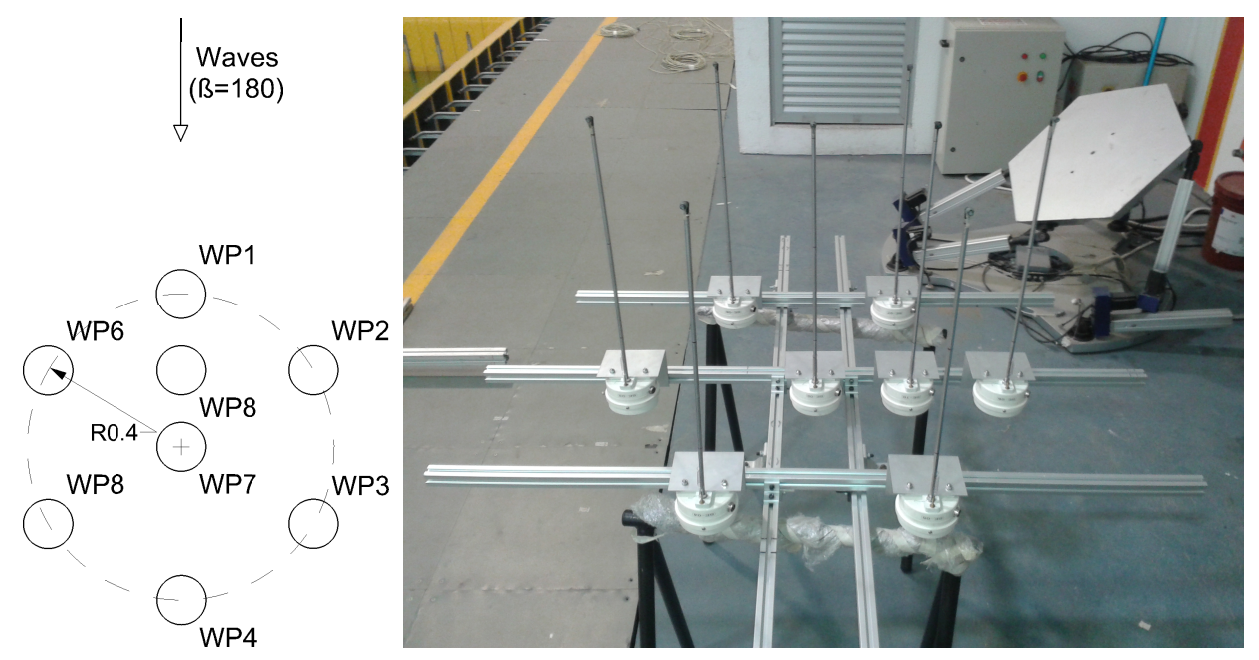

Figure 5.16: Lay-out of the wave probes array (left) and picture of the array before the installation in the wave basin (right).

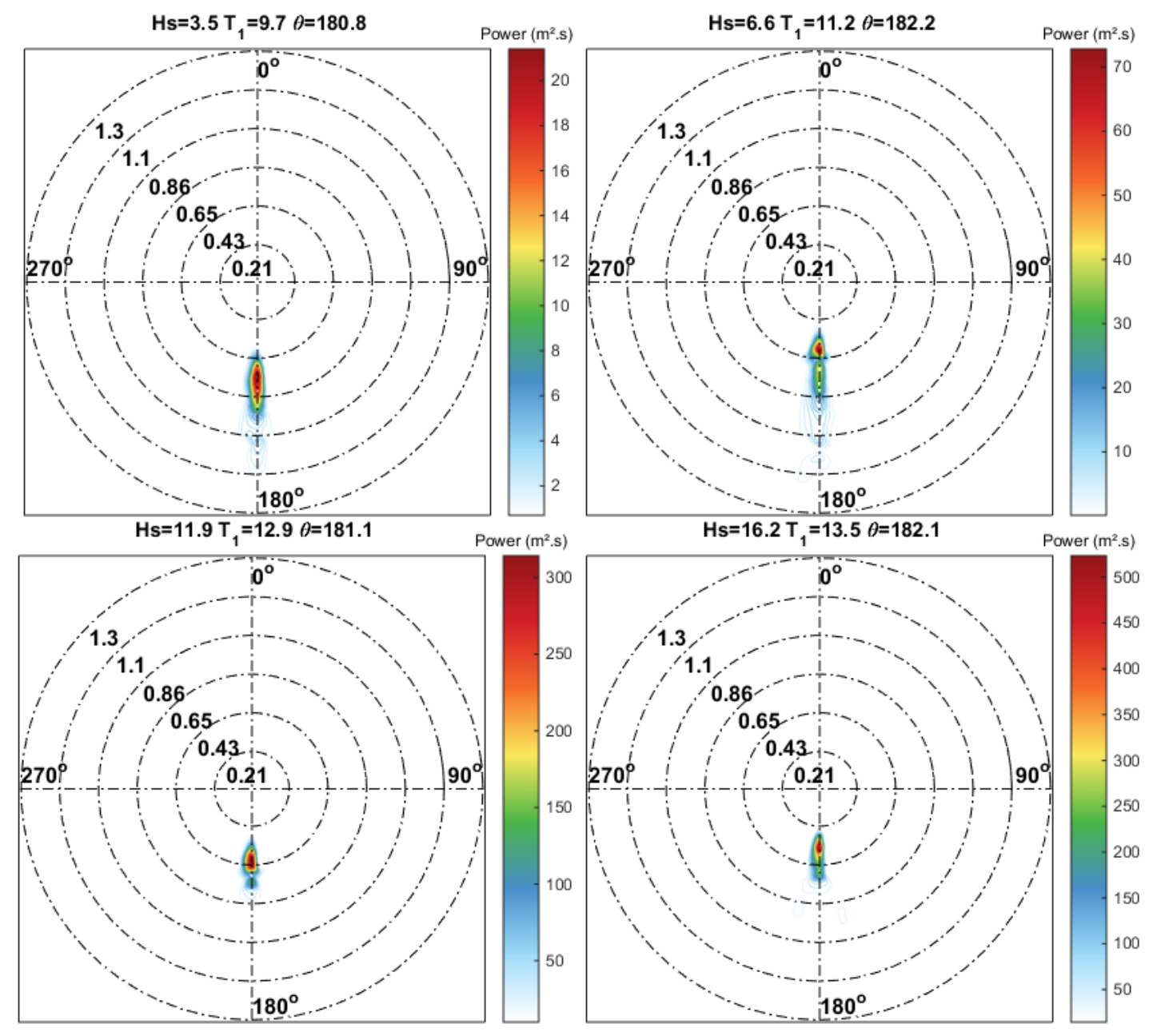

Figure 5.17: Irregular sea (full-scale) estimations using MEM for the tests: 2, 14, 25 and 32 (left-to-right then top-to-bottom). 


\subsection{Directional wave spectrum estimation based on model test data}

This section is divided in four different sections in order to arrange the results in a logical sequence. First, a discussion regarding the optimal sample time for each sea condition tested in the wave basin is provided in section 5.4.1. Section 5.4.2 contains the an assessment of the motion basis (heave-roll-pitch) adopted for wave inference. The analysis of the adequacy regarding the motion basis has been carried out based on coherence functions. Section 5.4.3 provides a comparison between the outcomes of the wave inference algorithm obtained using the different sets of RAOs proposed in section 5.2. The VMB wave inference method adopted in section 5.4.3 corresponds to the conventional approach, whose reliability to estimate different sea conditions has been shown by the results provided in different works (SIMOS et al., 2012; BISPO et al., 2016). Therefore, it provides a consistent framework to compare and select the most appropriate set of RAOs. In section 5.4.4, the main results obtained from this analysis are compared with the sea estimations determined by means of the alternative prior distribution proposed in chapter 3 .

\subsubsection{Selection of the optimal sample time for each sea condition}

Previously, in chapter 4, it has been pointed out that it is usually assumed that the stationary time span of a sea state may range between $20 \mathrm{~m}$ and $3 \mathrm{~h}$ (the reader is referred to (SOARES; FRICKE, 2011) for more details regarding the stationary time span of different sea conditions). Nonetheless, from a practical point of view, this assumption may lead to a large uncertainty regarding the ideal sampling time (or optimal run length) of a wave record, due to the wide interval of time that this assumption involves.

Since the main scope of this work do not include the analysis of this topic, only a brief discussion is included here and a more detailed assessment of the procedure followed to determine the run length of each test can be found in appendix B. In theory, the methodology followed should work properly if the measurement of the wave elevation of a stationary sea state is used as an input (once again, see appendix B). However, for fullscale applications in VMB wave inference, only the measured motions of the platform are 
provided. It has been shown in appendix B that the statistical properties of the motion spectra cannot be assumed stationary. Nonetheless, certain applications, such as the semisubmersible platform under study, that presents a fixed heading relative to the seabed and a constant operational draft, may provide reliable information about the optimal sample time through the measured motions. In order to provide a preliminary evaluation, the optimal sample time for each condition tested in the wave basin was estimated based on the measured motions of the model-scale platform during the experimental campaign.

This technique provides time series with the same statistical significance, since it aims at keeping the variance of a certain estimator always below a given value. As a result of the adoption of the measured heave motion as the input to estimate the sample time, the estimator provided by Eq. (B.16) has been selected as indicator. Figure 5.18 shows the coefficient of variability of the estimator for all the sea conditions tested in the wave basin as a function of the time, based on the measured heave motion of the model. Lines with the same color stand for the same sea condition, with the continuous line being the first realization and the dashed line the second realization of the same sea condition using a different phase seed, with a total time duration of 1.5 hour, each. As a consequence of keeping the model of the platform as simple as possible and the fact that the test matrix was composed by long crested wave conditions, the coefficient of variability of the temporal variance estimator selected was characterized by extremely low values. Accordingly, the threshold adopted to determine the sample time was given by a coefficient of variability of the temporal variance estimator equal to $1.2 \%$.

The resulting total sample time obtained for each test is provided in Fig. 5.19. In order to show a general idea about the results obtained through the analysis, this figure also includes the estimated mean value for all the test conditions $(41.5 \mathrm{~min})$ and the $25^{\text {th }}$ and $75^{\text {th }}$ percentiles, which are equal to $31.6 \mathrm{~min}$ and $56.5 \mathrm{~min}$, respectively. Also, the minimum time used for the analysis, corresponding to 30 minutes, and the maximum test time, 90 minutes, are included by the dashed blue and red lines. From this figure one may realize that the optimal sample time for extreme sea conditions will be larger than the sample time expected for calm sea conditions, as it is expected.

The largest sample time concerns the first realization of the test number 23. From Tab. 5.3, it is known that this sea condition was generated using a JONSWAP spectrum, 


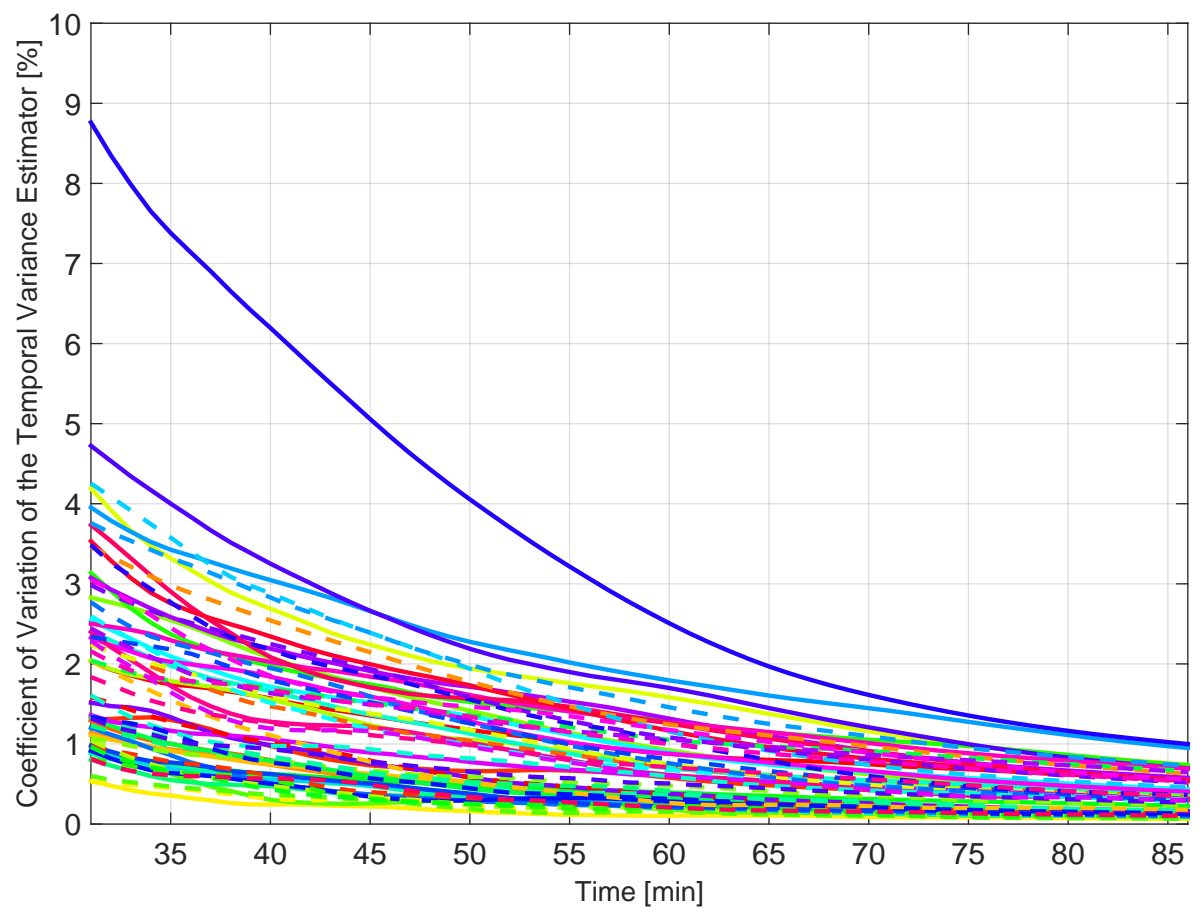

Figure 5.18: Coefficient of variability of the temporal variance estimator for all the sea conditions.

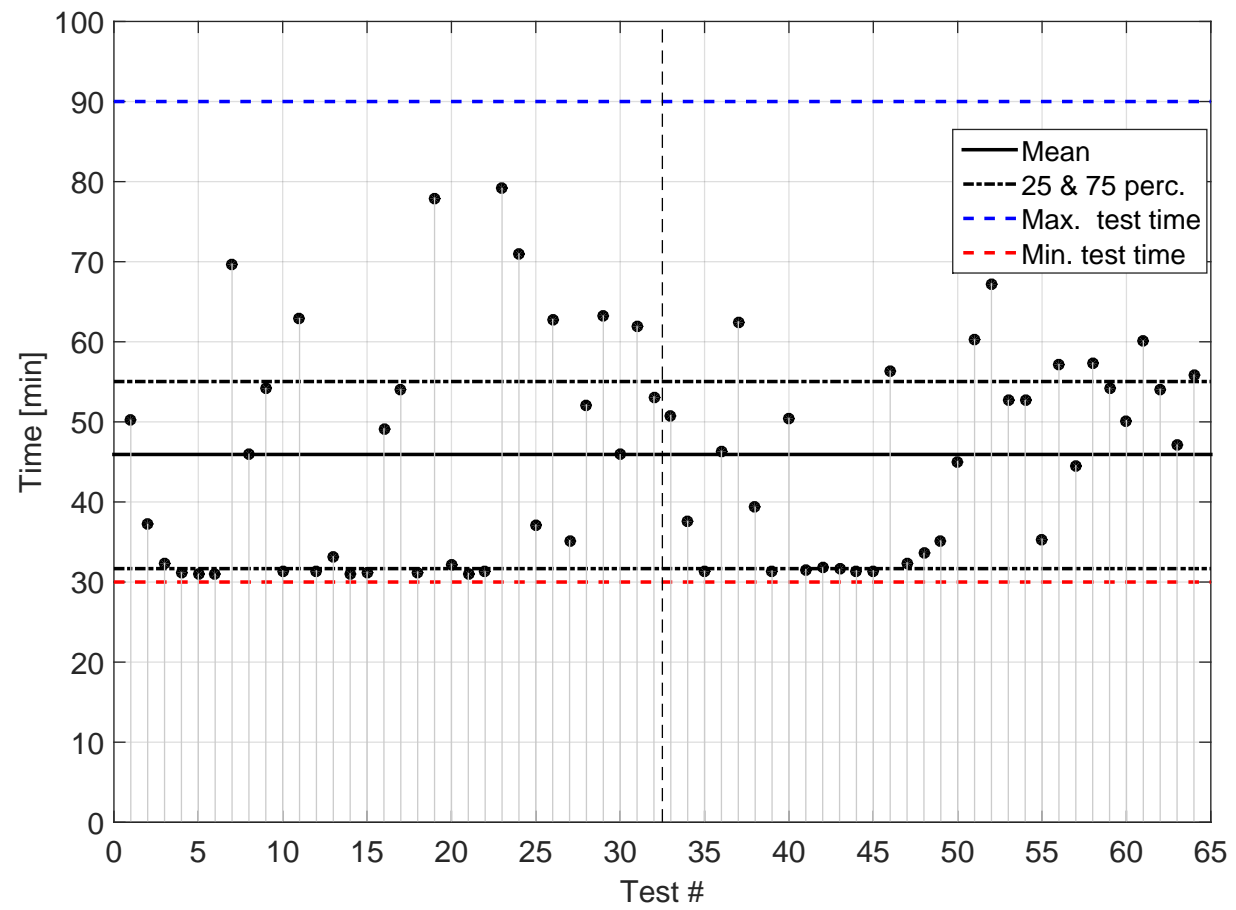

Figure 5.19: Optimal sample time for each sea condition.

with significant wave height equal to $10 \mathrm{~m}$ and peak period of $14.5 \mathrm{~s}$. Some examples of a comparison between the theoretical and the estimated spectrum obtained in each 
repetition are included in Fig 5.15. Test number 23 has been included among the different examples provided in Fig. 5.15, and it presents no significant deviation from the theoretical spectrum. Therefore, the large duration of the wave record can be associated to the random nature of the statistical properties of the motions spectra and the extremely low threshold adopted for the coefficient of variability of the temporal variance estimator $(1.2 \%)$.

In order to provide a general benchmark for the results presented in Fig. 5.19 the guidelines presented in (ITTC, 2014) have been followed. As a matter of fact, the guidelines proposed by the International Towing Tank Conference (ITTC) use the number of waves (encounters), $N$, obtained during the test to determine the optimal rung length of each test and can be summarized as follows: (i) $N=50$ should be taken as a lower limit; (ii) $N=100$ is accepted as a standard and (iii) $N=200$ or larger is considered as an excellent practice. In this case, for comparison purposes, $N=200$ to compute the optimal sample time. The results of this analysis shown in Fig. 5.20.

The results obtained following the approach proposed in (ITTC, 2014) are characterized by leading to lower sample values than the ones provided in Fig.5.19 (also included in Fig. 5.20). This is well illustrated by the mean value obtained for the outputs of the ITTC method (equal to $34.4 \mathrm{~min}$ ) as well as the $25^{\text {th }}$ and $75^{\text {th }}$ percentiles, equal to $31.4 \mathrm{~min}$. and 37.2min., respectively. Furthermore, the estimations obtained for some of the sea conditions tested during the experimental campaign show values even lower than 30min. Other characteristic of the outputs obtained following the ITTC guidelines is the strong correlation between the recommended sample time and the increase of the increment of the peak period, as expected. Moreover, the right side of the figure shows the histogram of the results obtained with a class interval equal to $1 \mathrm{~min}$, showing that the ITTC estimations have a narrower distribution that the ones presented in Fig. 5.19.

The results obtained in Fig. 5.20 attested that the methodology followed in this work to determine the sample time provides, in general, larger run lengths than the ones obtained through the ITTC guidelines. As a consequence, the procedure adopted results in sample times that can be considered indeed, following the guidelines described in (ITTC, 2014), as an excellent practice for the characterization of the sea conditions.

It is important to understand the possible effects of the sample time on the wave 


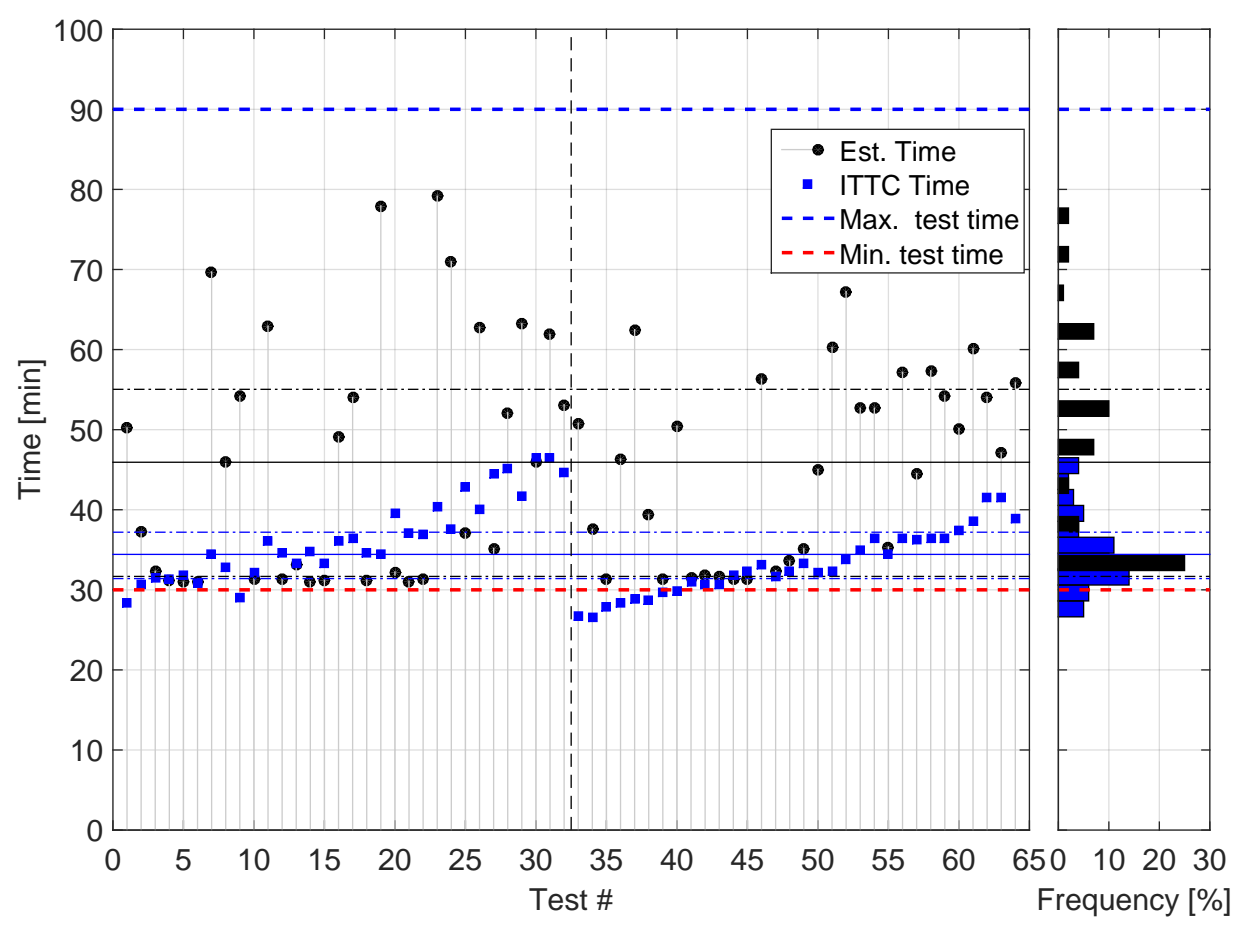

Figure 5.20: Comparison between the optimal sample time estimated for each sea conditions as proposed in Fig. 5.19 (Est. Time) and ITTC (ITTC Time). In this case the percentile levels for the results provided in Fig. 5.19 and the ones drawn using the ITTC approach are provided by the point-dashed lines (25\% and $75 \%)$ and continuous lines $(50 \%)$ in blue and black in black and blue, respectively.

inference. For the sake of illustration Tab. 5.4 has been included, which for the time being must be intended as quantitative comparison rather than a qualitative one. In this case the table provides the results obtained using the inference approach (which are analyzed with further detail in section 5.4.3), for sea condition 23 using three 30min time span intervals as well as the sample time estimated previously, i.e. 79.1min. Moreover, the measurements obtained using the wave probes (corresponding to wave record of $79.1 \mathrm{~min}$ ) are also included in this table as general reference for the estimations. From these results it can be stated that there are no significant differences between the estimations obtained regarding all the parameters evaluated (i.e. significant wave height, peak period and mean wave direction) when using different time lengths and intervals. Nonetheless, it is important to highlight the fact that as the test progresses there is a tendency that results in slight increments of the wave statistical values. This effect may be related to the small amount of energy that is not absorbed by basin absorbtion system (see (MELLO et al., 2013)). However, it shows that if the stationary condition is not accurate for short time 
intervals (see for instance (HAVER; MOAN, 1983)) the adoption of short sample time may lead to misleading estimations of the sea states.

In this work the methodology described above, and which results are presented in Fig. 5.19, has been followed to estimate the optimal sample time to estimate the sea states. This approach will ensure similar statistical significance of the results and, at the same time.

Table 5.4: Comparison between the results obtained using 30min intervals over the entire span of the test (90min) and the time estimated in Fig. 5.19 for test 23 (equal to $79.1 \mathrm{~m}$ ).

\begin{tabular}{ccccccc} 
& & \multicolumn{5}{c}{ Run Length } \\
\cline { 3 - 6 }$\beta$ & & $1-30$ min & $30-60$ min & $60-90 \min$ & 79.1 min & Measured \\
\hline \multirow{3}{*}{90} & $H_{s}$ & 9.21 & 9.6 & 10.51 & 9.9 & 10.27 \\
& $T_{p}$ & 12.7 & 12.4 & 12.6 & 12.6 & 11.7 \\
& $\theta$ & 179.6 & 179 & 177 & 180 & 180 \\
\hline \multirow{3}{*}{120} & $H_{s}$ & 9.14 & 9.6 & 10.24 & 9.9 & 10.27 \\
& $T_{p}$ & 12.83 & 12.44 & 12.54 & 12.6 & 11.7 \\
& $\theta$ & 178.7 & 177 & 179 & 178 & 180 \\
\hline \multirow{3}{*}{135} & $H_{s}$ & 10.14 & 10.43 & 10.71 & 10.4 & 10.27 \\
& $T_{p}$ & 12.89 & 12.47 & 12.15 & 12.5 & 11.7 \\
& $\theta$ & 177.9 & 178 & 179 & 179 & 180 \\
\hline \multirow{3}{*}{150} & $H_{s}$ & 9.42 & 9.74 & 10.1 & 9.9 & 10.27 \\
& $T_{p}$ & 12.8 & 12.46 & 12.54 & 12.6 & 11.7 \\
& $\theta$ & 177.9 & 178 & 178 & 180 & 180 \\
\hline \multirow{2}{*}{180} & $H_{s}$ & 9.29 & 9.79 & 10 & 9.7 & 10.27 \\
& $T_{p}$ & 12.88 & 12.7 & 12.88 & 12.8 & 11.7 \\
& $\theta$ & 179.5 & 179 & 179 & 179 & 180 \\
\hline
\end{tabular}

Regarding full-scale applications, however, this approach is expected to present several limitations. First, usually only the time motion time series are recorded (the use of full-scale waves probes still, generally, limited to few research projects). Secondly, it is already known that the sea surface elevation time series (as well as the motion time series) often result in a non-stationary process, thus (in theory) the run length value estimated thought this technique may not be optimal. Finally, the results of this method may vary in accordance with the threshold value selected. For instance, if it is increased to $5 \%$, Fig. 5.18 shows that the expected sample duration for all the sea conditions tested would be $40 \mathrm{~min}$., except for the test 23 that would present a run length equal to $45 \mathrm{~min}$. Therefore, for full-scale applications, the common practice of adopting 30min intervals may be considered reasonable. 


\subsubsection{An analysis of the adequacy of the heave-roll-pitch mo- tion basis for VMB wave inference based on coherence functions}

Previously, at the beginning of this chapter, it has been pointed out that the VMB wave inference will be made based on the three motions, namely heave, roll and pitch. The adoption of this basis is primarily due to the analogy with wave buoys, which usually take into account the set of motions with vertical movements as a base for estimation the sea states (see, for instance (TANNURI et al., 2003)). Next, the adequacy of this basis (heave, roll and pitch) is evaluated by means of coherence functions.

The degree of linear dependency between two signals can be quantified by means of the coherence function, which provides a measurement of the causality between the input and the output of a linear system. For the purpose of this thesis, it stands as an assessment of the linearity between the incoming wave, $\eta$, and motion response, $\xi$. Regarding the output of this function, it varies between zero and one. Therefore, if $\eta$ and $\xi$ are uncorrelated the sample coherence converges to zero. On the other hand, values equal to one indicate linearity between the excitation and the response.

The coherence function between $\eta$ and $\xi$ for a certain frequency, $f^{*}$, is defined as follows (BENDAT; PIERSOL, 2010),

$$
\gamma_{\eta \xi}\left(f^{*}\right)=\frac{S_{\eta \xi}\left(f^{*}\right) S_{\xi \eta}\left(f^{*}\right)}{S_{\eta \eta}\left(f^{*}\right) S_{\xi \xi}\left(f^{*}\right)}
$$

where $S_{\eta \xi}\left(f^{*}\right) S_{\xi \eta}\left(f^{*}\right)=S_{\eta \xi}\left(f^{*}\right) S_{\eta \xi}^{*}\left(f^{*}\right)=\left|S_{\eta \xi}\left(f^{*}\right)\right|^{2}$. Thus,

$$
\gamma_{\eta \xi}\left(f^{*}\right)=\frac{\left|S_{\eta \xi}\left(f^{*}\right)\right|^{2}}{S_{\eta \eta}\left(f^{*}\right) S_{\xi \xi}\left(f^{*}\right)},
$$

Figure 5.21 and Fig. 5.22 show the coherence function values computed for the six motions of the semisubmersible platform. These figures include the results obtained for all the 32 sea conditions tested in wave basin for beam and quartering sea conditions, $\beta=90 \mathrm{deg}$. \& $\beta=135 \mathrm{deg}$. respectively. Due to the geometrical properties of the hull of the Åsagrd-B platform, the results shown in these figures provide sufficient information about the responses of the platform regarding other heading conditions. 

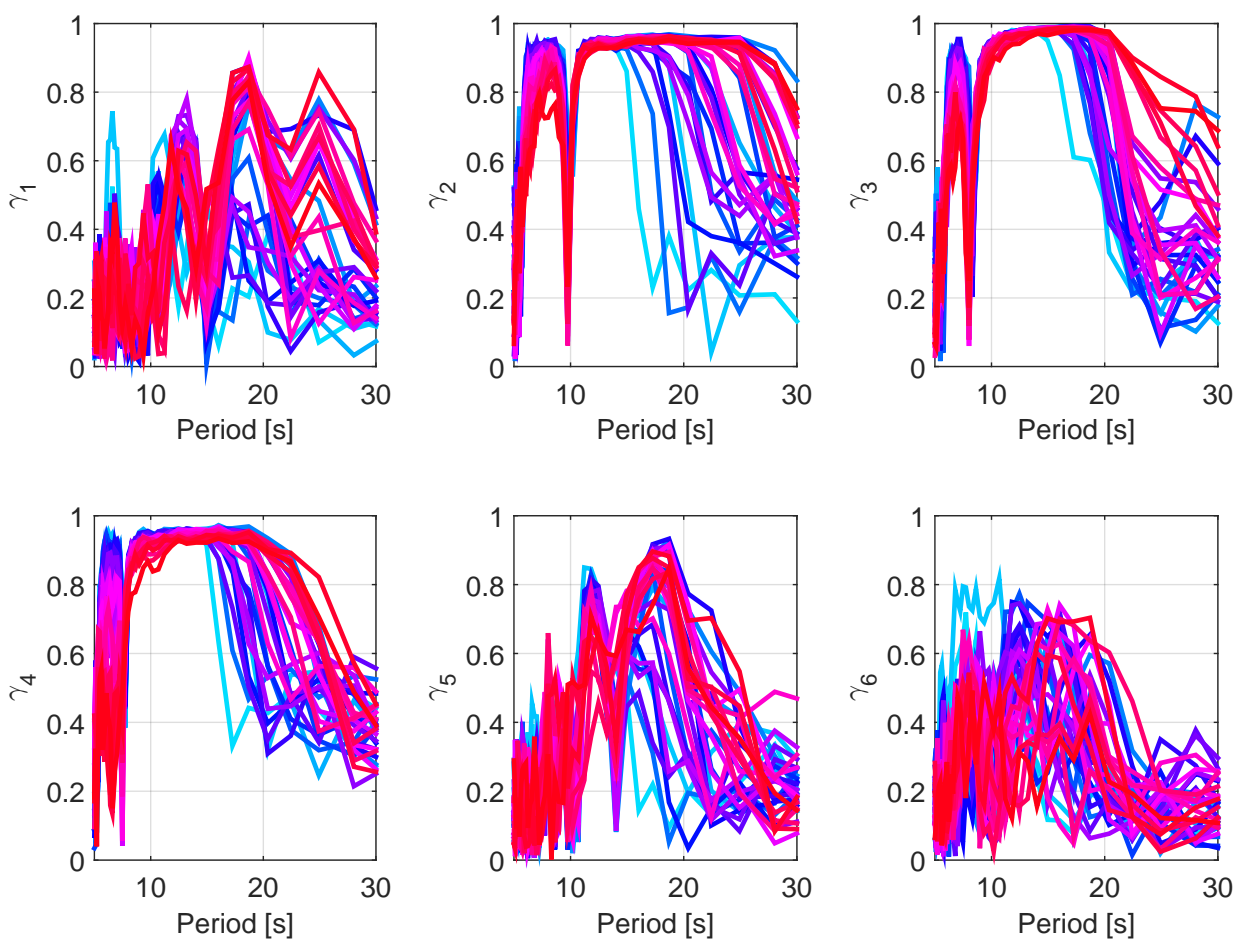

Figure 5.21: Coherence function for beam sea conditions.
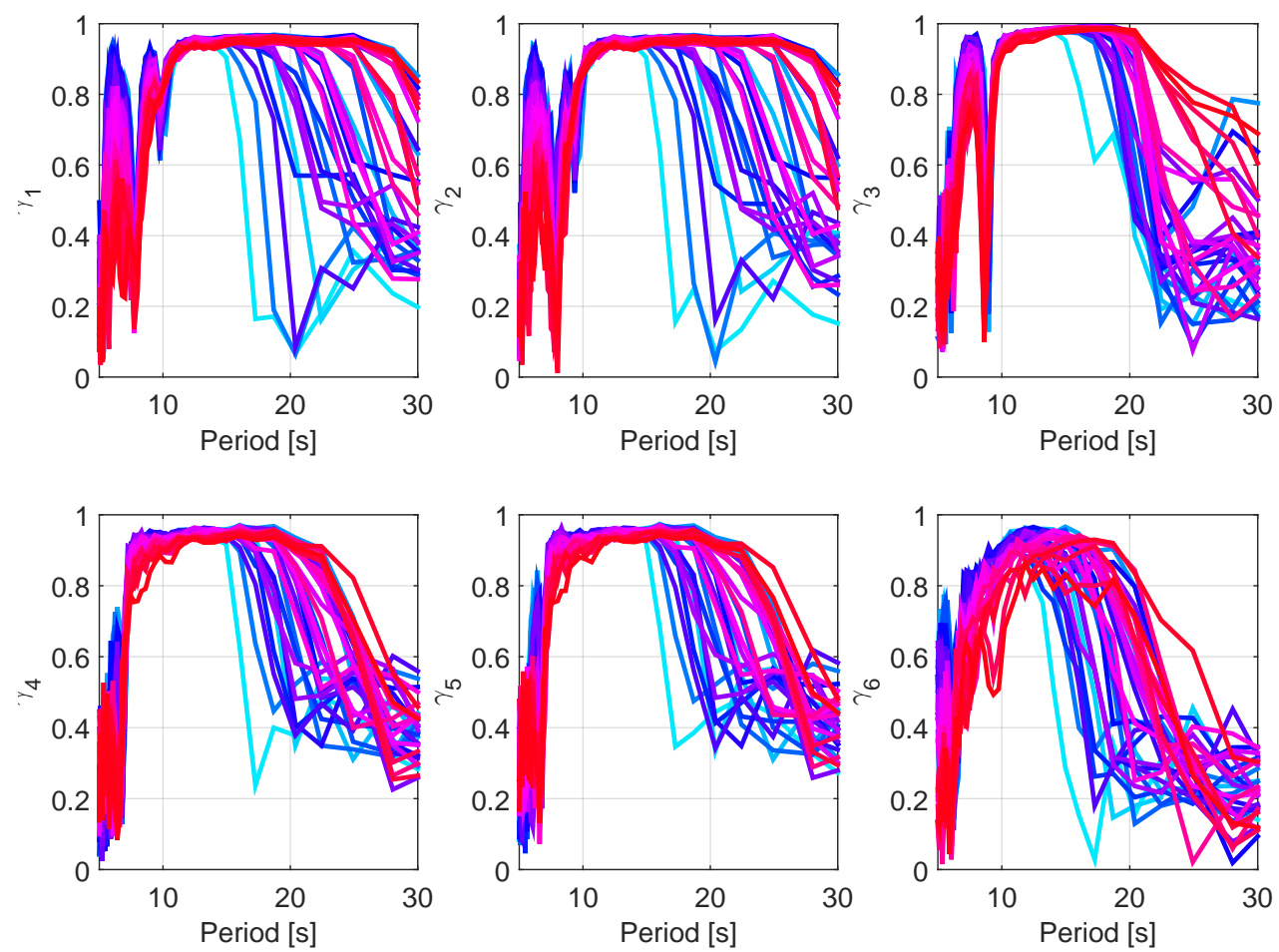

Figure 5.22: Coherence function for quartering seas.

Figure 5.21 shows that the coherence functions of sway, heave and roll motion are close to one in periods between $10 \mathrm{~s}$ and $20 \mathrm{~s}$. Hence, it shows that linear dynamics are 
indeed dominant in sway, heave and roll motions in this frequency range. Nonetheless, the values of the coherence function concerning the surge, pitch and yaw motions indicate lack of linearity between the excitation and the responses. This is due to the fact that this motions (in theory) are not excited in beam sea conditions. Therefore, the outputs of the coherence functions are expected to be lower than one.

Returning to the sway, heave and roll coherence functions presented in Fig. 5.21, one may realize that close to 10 s all of them present a local minimum, indicating that low linearity is expected between the excitation and the responses. Aiming to properly illustrate the origin of this minimum Fig. 5.23 has been included. This figure shows the coherence functions concerning tests 02 and 31 for the heave motion (in red), for quartering sea conditions. Moreover, the spectrum for each sea condition (blue line) and the theoretical heave RAO (black line) are provided. It is possible to identify by simple comparison that the minimum that the coherence function presents close to 10s is related to the cancellation point that the RAO presents at the same period. Similarly, the minima in the sway and roll motions in this period range are related to the zero points of the RAO (see Fig. 3.10). The values obtained of the coherence functions for the high period range $(T>20$ s) also show low values, which can be related to the small amounts of energy that the wave spectra tested present in this period interval. It is important to highlight the fact that the heave coherence functions do not present a minimum close to the cancellation point of the heave RAO in 22s. As it has been show in section 5.4.1 it is expected, due to non-linear drag effects, that the platform will present motions with significant amplitudes close to this period. Therefore, the resulting values of the coherence functions indicate that non-linear responses are expected.

The coherence functions for model tests in quartering sea condition presented in Fig. 5.22 show that linear effects are dominating in periods between $10 \mathrm{~s}$ and $20 \mathrm{~s}$, for all the dofs assessed. These results are in accordance with the previous analysis regarding carried out using the result presented in Fig. 5.21 and 5.23.

The analysis of the linear dynamic model provided above allows the assessment of adequacy of motion basis selected for VMB wave inference. First, it is important to state that the yaw motion in this case was not considered for VMB wave inference due to the uncertainty regarding the full-scale inertia properties of the platform around the $\mathrm{z}$ 

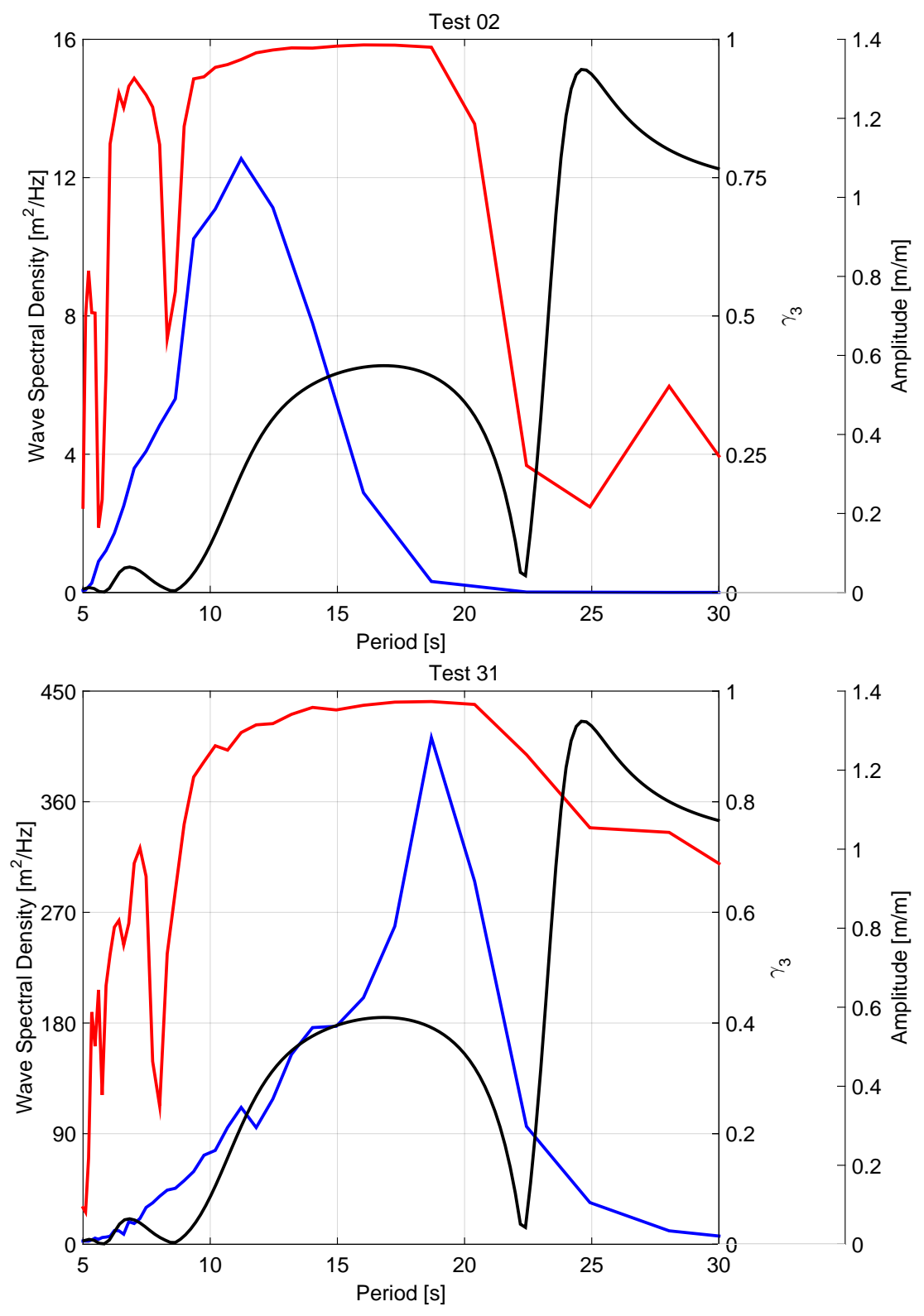

Figure 5.23: Comparison between the sea spectrum (in blue) for tests 2 and 31, top and bottom respectively, the heave coherence function (red) and the heave RAO (black).

(vertical)-axis ${ }^{6}$. From the previous Fig. 5.21 and 5.22 one may realize that the use of the surge and sway motions could provide relevant information about some sea conditions in the low frequency range. However, for beam and head sea conditions, these will result in that at least two dofs of the base adopted to estimate the sea conditions are expected to present no significant linear wave excitations. As a consequence, these motions will be predominantly driven by other factors, e.g. as winds, currents or even low frequency waves drifting forces (WIJAYA, 2009). Therefore, the use of the 5 motions as the base to

\footnotetext{
${ }^{6}$ The properties that the scale model of the platform presented were properly modelled for the numerical analysis.
} 
estimate the sea conditions, for this semisubmersible platform, will result in the escalation of the uncertainties inherent to the problem. Consequently, the base of motions selected to estimate the sea conditions based on the measured motion of a semisubmersible platform will be composed by: Heave, roll and pitch.

Finally, another use of the coherence function is the one proposed by Nielsen (2005). The main idea is to use the coherence function as a normalization factor for the measured motions of the floating vessel. This approach guarantees that motions with strong linearities in a certain frequency range will be advantaged at the expense of the ones that are expected to present no significant linearities. However, the lack of the physical quantification of the motion (it does not provide comparative values for different motions such as heave and pitch responses) of this technique usually leads to misleading estimations.

The method followed in this thesis normalizes the motion signals by means of using the main dimensions of the platform. Therefore, the angular motions will be multiplied by a certain linear dimension, i.e. the half beam of the platform or the half length, for the roll and pitch motions, respectively. This procedure does not guarantee that all the recorded motions will present the same uncertainty (because the RAOs vary in the frequency range). Nevertheless, the alternative methodology to deduce the prior distribution proposed in section 4.4.2 improves this shortcoming by using the measured motions and increasing the uncertainty of the estimation close to the point of minima of the RAOs.

\subsubsection{Wave inference using the conventional prior distribution}

In the following, the results obtained with the wave inference method based on the motions of the semisubmersible model are presented and confronted with the measurements performed in the wave basin with conventional wave probes, with the following objectives: (i) to evaluate the impact of the viscous drag forces on the RAOs, as discussed in chapter 3 and (ii) to provide a general assessment of the use of a large semisubmersible as a VMB wave sensor.

Accordingly to the results provided in section 5.4.2, the motion base adopted for the analysis comprises only three motions, namely: Heave, roll and pitch. On the one hand, three different sets of RAOs were employed as alternatives for modelling the heave motions: One with a constant external damping coefficient corresponding to $5 \%$ of the 
critical damping, which is a typical value for the external viscous damping of this kind of floating unit and is in accordance with the results obtained through the experimental decay tests of the heave motion. Secondly, the set of RAOs resulting of the variable damping method in heave computed following the procedure proposed in section 5.2. In the third place, aiming at providing a reference framework, the RAOs derived from time-domain simulations in the software ANSYS-AQWA with a wave steepness equal to $2 \%$, which includes the pontoon drag forces (see Fig. 5.6). As a matter of fact, this heave motion RAO has been adopted to assess the possible impact that the non-linear effects may have on the estimations. On the other hand, the roll and pitch motions are characterized by high resonant periods (see section 5.1). Therefore, these motions were modelled for all the cases by means of the RAOs estimated through the frequency-domain analysis. Finally, in order to estimate the sea conditions, twenty different wave frequencies were considered, with a spatial resolution of $\Delta \beta=5$ degrees (corresponding to $\mathrm{M}=20$ and $\mathrm{K}=72$, see chapter 4 ).

Regarding the values of the hyperparameters, an extensive sensitivity analysis has been performed in order to obtain the optimal values. Thus, it was determined that: $u_{1}$ remains equal as the one proposed in (BISPO, 2011a), where the author proposes the estimation of the value of the first hyperparameter by means linear interpolation. For instance, Bispo (2011a) interpolates the value of $u_{1}$ within a range given by the interval [0.00208 0.02000] and using the mean zero up-crossing period of the heave motions, which must range between $7.5 \mathrm{~s}$ and $14 \mathrm{~s}$. In those cases where the estimated mean zero up-crossing period is larger than $14 \mathrm{~s}, u_{1}$ is assumed equal to 0.02 . For $u_{2}$, the optimal value is given by $1 \cdot 10^{-5}$ and $u_{3}$ is set to 0.0035 . The small value of $u_{2}$, if compared with the value provided in (BISPO et al., 2012), arises from the narrow differences between the beam and length of the platform, which produce complementary RAOs for roll and pitch motions. This resemblance maintains all the zero points of the roll and pitch RAOs limited to certain frequency ranges and, therefore, larger values of $u_{2}$ are no longer needed.

From a practical point of view, it is also important to remind that the VMB wave estimations were made considering the acquisition time determined in section 5.4.1, following the procedure provided in appendix B.

For all the results shown ahead, the computations were carried out with a desk- 
top personal computer using MATLAB ${ }^{\circledR} 2014 \mathrm{a}$, which contains the toolbox solver $^{7}$ for numerical optimization that was used to estimate the minimum of the objective function derived from the Bayesian approach.

In Figs. 5.24 to $5.27^{8}$ the reliability of the estimations was evaluated by means of the relative errors. Accordingly, the vertical axes of these figures provide the relative errors of the following statistics:

- Significant wave height $\left(H_{s}\right)$, whose relative error is given by,

$$
\eta_{H_{s}}=\frac{H_{m}-H_{s}}{H_{m}},
$$

where $H_{m}$ stands as the measured significant wave height and $H_{s}$ is the value estimated from the model motions;

- Mean wave period $\left(T_{1}\right)$, that leads to the following relative error,

$$
\eta_{T_{1}}=\frac{T_{m}-T_{1}}{T_{m}},
$$

where $T_{m}$ stands as the measured mean wave period and $T_{1}$ is the estimated value of the mean wave period. It must be emphasized, once again, that the platform is expected to filter certain part of the energy placed in the high frequency range. For this reason, $T_{1}$ has been adopted instead of $T_{p}$, for being a better indicator of the distribution of the energy.

The relative errors in the wave statistics are presented as a function of the (measured) mean period $\left(T_{1}\right)$ of the waves in each test, given in the lower horizontal axis. The upper axis indicates the corresponding (measured) significant wave heights, in meters, so that the reader can easily assess the agreement obtained with the VMB wave inference method

\footnotetext{
${ }^{7}$ In this case the optimization toolbox adopted uses the quadprog as a solver.

${ }^{8}$ The prediction of the mean direction was very accurate for all the conditions tested. Therefore, only the relative errors regarding the significant wave height and the mean wave period are provided in these figures. Regarding the mean wave direction $\theta$, the relative error may be written as,

$$
\eta_{\theta}=\frac{\theta_{s}-\theta_{m}}{\pi},
$$

where $\theta_{m}$ stands as the measured mean wave direction and $\theta_{s}$ is the estimated value of the significant wave height.
} 
for different wave periods and heights. Moreover, it should be noticed that these figures present three sets of results, that correspond to the estimations obtained using the RAOs described above.
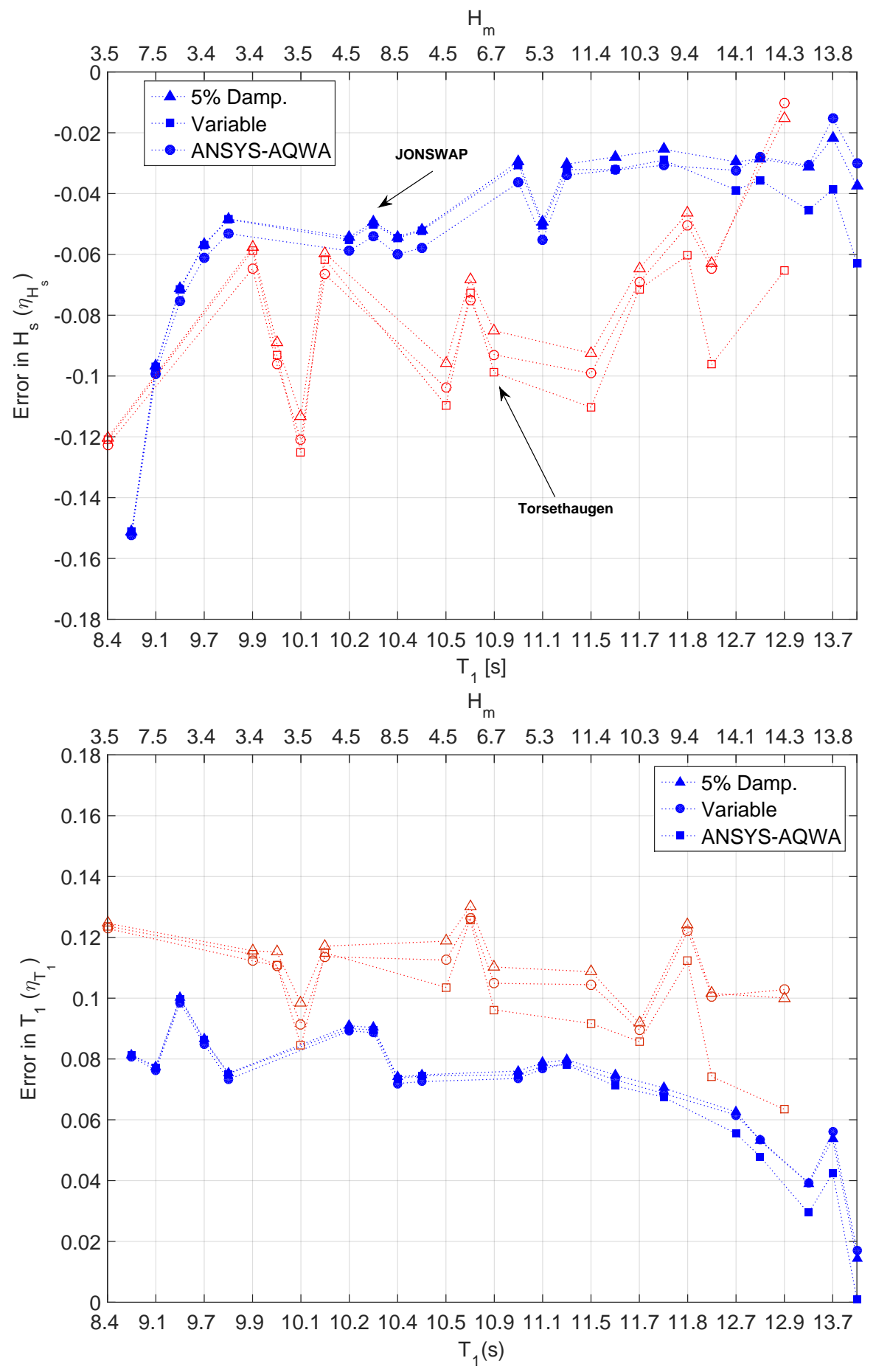

Figure 5.24: Results of the wave inference method estimated with different sets of RAOs for the heading $90^{\circ}$, concerning the first realization. Blue and red markers indicate whether the waves generated in the basin corresponded to a JONSWAP or Torsethaugen power spectrum, respectively. The relative errors provided in these figures are defined in Eqs. (5.9) and (5.10). 

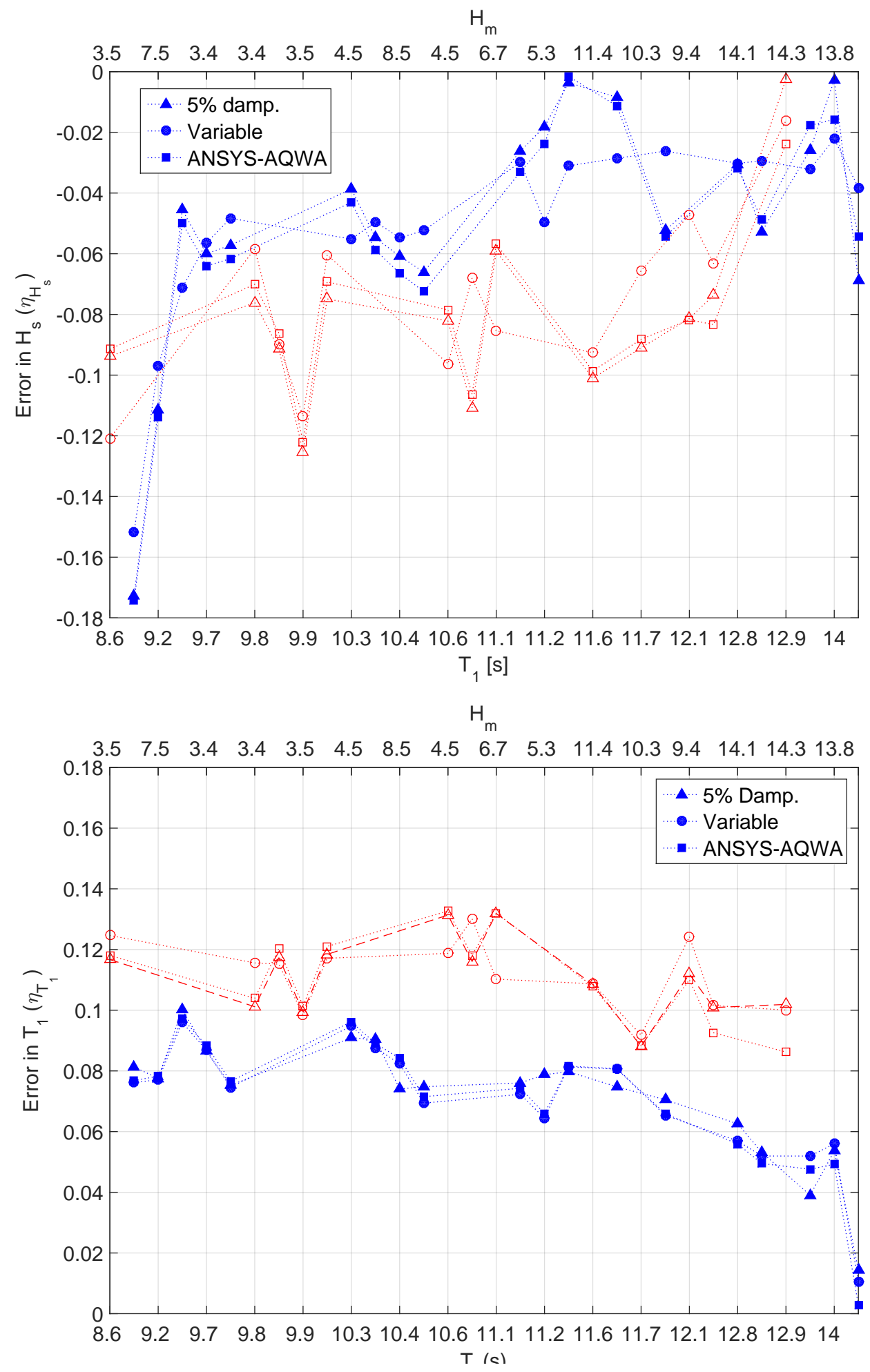

Figure 5.25: Results of the wave inference method estimated with different sets of RAOs for the heading $90^{\circ}$, concerning the second realization. 

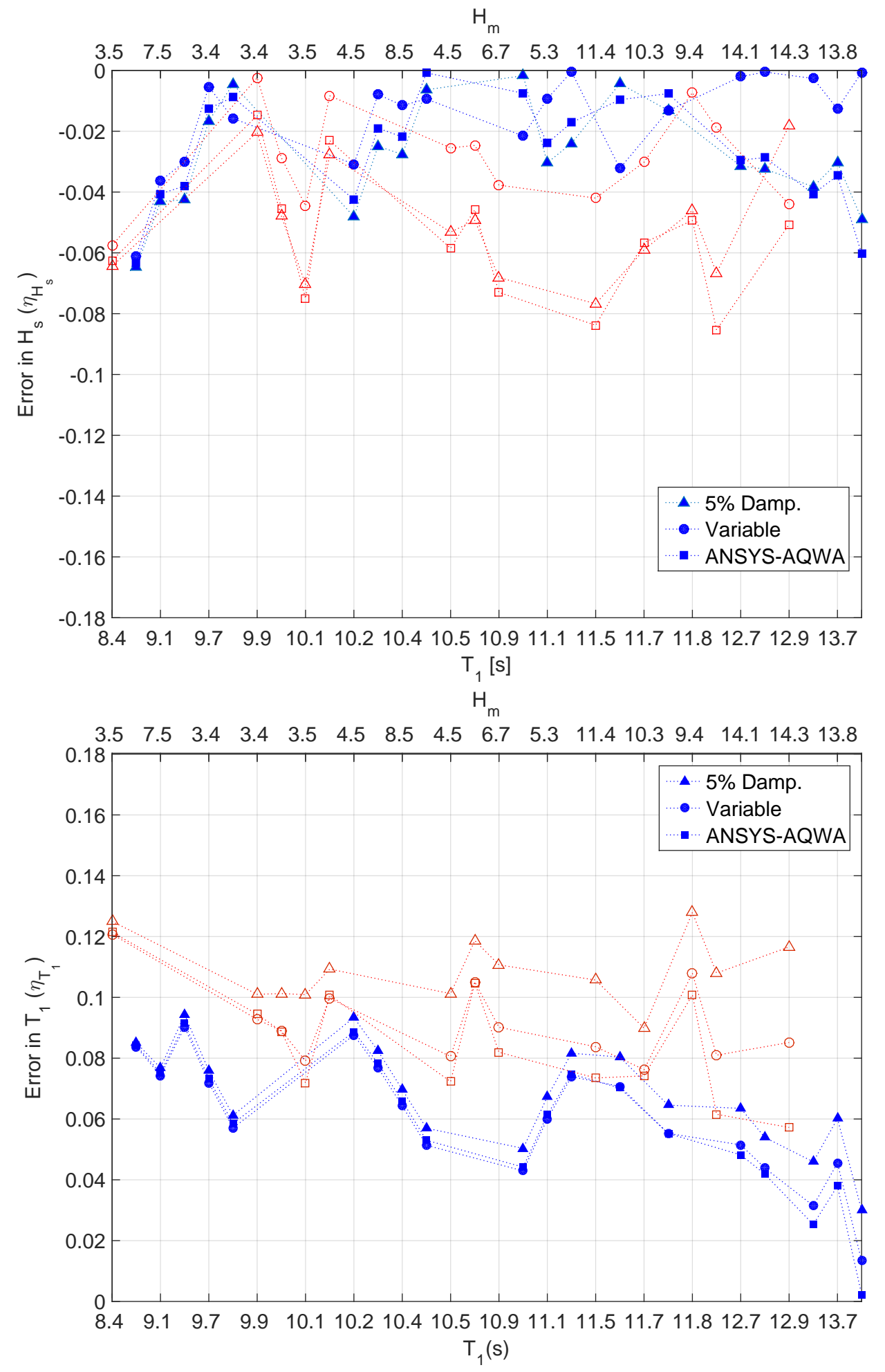

Figure 5.26: Results of the wave inference method estimated with different sets of RAOs for the heading $135^{\circ}$, concerning the first realization. 

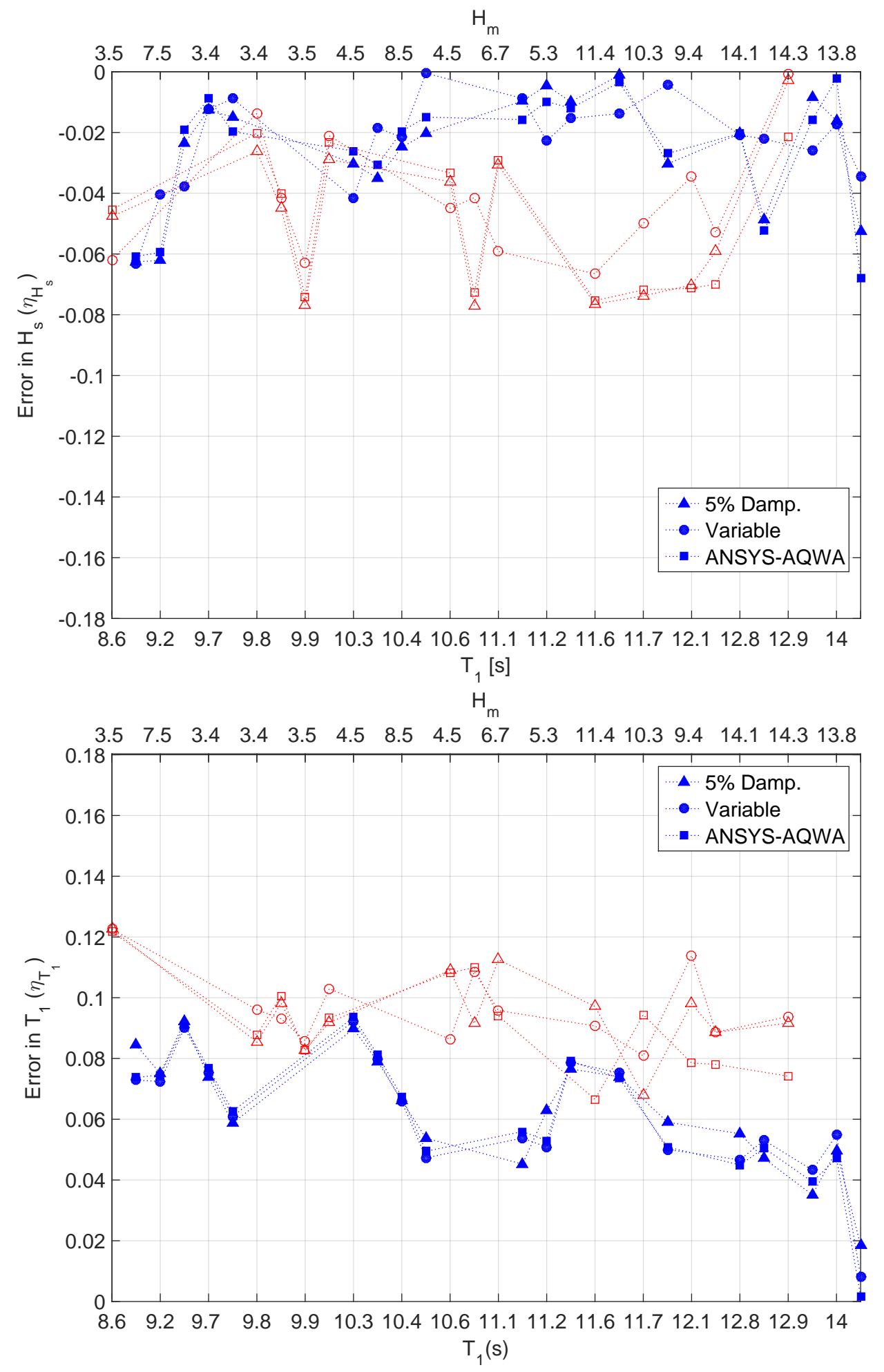

Figure 5.27: Results of the wave inference method estimated with different sets of RAOs for the heading $135^{\circ}$, concerning the second realization.

It must be realized that the results are clustered into two different sets with different colors, indicating whether the waves generated in the basin corresponded to a JONSWAP 
(blue) or Torsethaugen (red) power spectrum.

Finally, one should also notice that the figures referred above only include information for waves coming from $90^{\circ}$ and $135^{\circ}$. The reason for this is that the results obtained for other heading conditions tested during the experimental campaign do not provide any meaningful new information, as it will be shown ahead in section 5.4.3. For each heading, two analogous figures are included, corresponding to the two realization of the same sea conditions carried out in the experimental campaign. Based on these figures, a detailed discussion of the performance of the VMB wave inference method will be presented next.

\section{Discussion}

Many aspects of the results presented above deserve attention. First, by inspecting the error statistics obtained with the wave inference method, it can be concluded that the overall agreement with the expected values is indeed very good for all the headings analyzed.

Regarding specifically the significant wave height, the inference method tends to underestimate the experimental values, with errors always below $18 \%$ for the most unfavorable heading $\left(90^{\circ}\right)$ and only $9 \%$ for the most favorable one $\left(135^{\circ}\right)$. It must be emphasized that the underestimation observed is indeed consistent with the expected bias of the method, since part of the energy in the high frequency wave components is filtered as these waves do not impose large motions on the platform. It is also important to observe that the level of underestimation and the difference in this level for the different headings tend to decrease as the wave period increases, thus favoring the results for the most severe sea states.

The results obtained for the predicted mean wave periods $\left(T_{1}\right)$ are also consistent with the bias of the method. Since energy is filtered from the wave components with higher frequencies, the mean frequency of the estimated spectrum tends to be lower than the real one and, consequently, the mean period tends to be higher. Nonetheless, the results show that differences are not large, with errors below $15 \%$ in all cases. Once again, the errors decrease for larger wave periods, as should be expected.

Figures 5.24 to 5.26 also allow an assessment of the influence of the different heave $\mathrm{RAO}$ models on the VMB predictions. It is interesting to realize that the main influence 
arising from the consideration of pontoon drag effects occurs in the estimation of the significant wave height. Moreover, the discrepancies in the wave height predicted by the different models increase for larger periods. This was already expected and happens due to the fact that these waves induce resonant heave motions, where the three sets of RAOs adopted behave differently. Nonetheless, it is important to highlight that the results obtained for the Torsethaugen wave spectrum show larger discrepancies between the three models. This is due to fact that in general the Torsethaugen condition are estimated with less accuracy than the JONSWAP sea condition, as a consequence of filtering the high frequency components of the spectrum. Therefore, small changes in the low frequency range lead to more relevant differences than in sea states generated by means of the JONSWAP sea spectrum.

Since the results provided in this figures are characterized by a large variability, which makes it difficult to identify a clear tendency among the results obtained, a more detailed analysis of the results provided in the figures above is done next.

As pointed out in section 5.2.3 and in the previous section, the set of RAOs derived from the time-domain simulations allows a sensitivity analysis of the effects that non-linear responses may have on the estimation. However, the usefulness of the RAOs obtained numerically is limited due to the fact that the main characteristics of the sea conditions should be known a priori in order to compute the most appropriate set of RAOs. Consequently, only the sets of RAOs obtained through the variable damping model or assuming a constant damping model are eligible, in practice. It is worth remembering that the RAOs derived from time domain simulations have been included in order to provide a reference benchmark, thus allowing an early assessment of the possible effect that may arise if non-linear dynamics are take into account. For instance, the results obtained show that large discrepancies are not expected.

One may find that the results in the Figs. 5.24 to 5.27 are somewhat inconclusive when it comes to selecting the most appropriate set of RAOs. Therefore, aiming to provide all the data in a suitable and manageable form, Tab. 5.5 is included herein. This table provides the mean value of the relative errors (regarding the significant wave height and the mean wave period) obtained from both headings analyzed and the two sea realizations. Also, the results are clustered by the model of RAOs used. The differences between the 
results predicted adopting different models increase for larger periods, due to the induced heave motions near the resonance. Therefore, only the 10 sea conditions with the largest mean wave periods have been included in the table.

Table 5.5: Mean of the relative errors measured during the experimental campaign for $H_{s}$ and $T_{1}$, regarding the ten sea conditions with the largest mean wave periods.

\begin{tabular}{lcc|cc}
\cline { 2 - 5 }$T_{1}$ & \multicolumn{2}{c|}{$\eta_{H_{s}}$} & \multicolumn{2}{c}{$\eta_{T_{1}}$} \\
\cline { 2 - 5 } 11.7 & Var.Damp. & $5 \%$ Damp. & Var.Damp. & $5 \%$ Damp. \\
11.77 & 0.0669 & 0.0774 & 0.0907 & 0.0908 \\
11.8 & 0.0485 & 0.0382 & 0.067 & 0.0705 \\
12.41 & 0.0638 & 0.068 & 0.1231 & 0.1181 \\
12.7 & 0.031 & 0.0301 & 0.0592 & 0.1012 \\
12.9 & 0.0283 & 0.0403 & 0.0527 & 0.0526 \\
12.93 & 0.0128 & 0.0084 & 0.1009 & 0.101 \\
13.65 & 0.0274 & 0.0279 & 0.0356 & 0.039 \\
13.73 & 0.0185 & 0.0118 & 0.056 & 0.0536 \\
14.7 & 0.0338 & 0.0531 & 0.0138 & 0.0143 \\
\hline
\end{tabular}

The results provided in Tab. 5.5 show that the damping models adopted to compute the RAOs of the semisubmersible platform do not lead to significant differences between the estimations of the sea conditions. Although the variable damping model in general provides slightly smaller values for the relative errors of the significant wave height and mean wave period than the constant damping model (in Tab. 5.5) none of these models can be conclusively selected as the most accurate. However, due to the practical advantages that the variable damping model provides, such as the fact that it does not rely on any experiment tests, it will be adopted in this thesis.

For a first assessment of the other statistical parameters, namely the mean wave direction and the wave spreading, Figs. 5.28 and 5.29 provide the directional wave spectra estimated for the same sea conditions previously displayed in Fig. 5.17, for the two different heading conditions. These spectra were estimated using the set of RAOs computed by means of the variable damping model. In these figures, the accuracy regarding the estimation of the mean wave direction $(\theta)$ can be assessed reminding that the expected wave direction in the wave basin is always $180^{\circ}$. Although all these examples are provided for illustration purposes due to the fact that only long crested wave conditions were generated in the basin, more results of the directional sea spectrum obtained by means the conventional approach can be assessed in Figs. 5.39 and 5.40. 
The spreading of the measured wave spectrum is larger than the one estimated by means of wave probes and the using MEM. The larger spreading of wave energy when using the Bayesian inference method is related to the calibration of the hyper-parameter $u_{1}$, which controls the level of smoothness of the spectrum with respect to the wave direction. In this case, the pre-calibration of the hyparameter was made seeking to improve the accuracy in the prediction of the main statistical wave parameters $\left(T_{1}, H_{s}\right.$ and $\left.\theta\right)$, a procedure that ultimately tends to lead to a larger wave spreading. Notwithstanding this, the sea conditions tested during the experimental campaign (long crested) do not stand as the most appropriate sea condition to assess the reliability of the direction spreading. As a matter of fact, the prior distribution adopted results from the assumption that the sea spectrum must be smooth in frequency and direction. Thus, the long crested sea conditions tested during the experimental campaign stand as limiting conditions for the wave inference method adopted (see, for instance section 4.4).

The results in Figs. 5.24 to 5.27 show that the spreading increases for sea conditions characterized with higher peak periods. This is due to the fact that in severe sea conditions the amount of energy close to the zero point of the heave RAO (around 22s) is larger than in mild/calm sea states. Therefore, in these conditions the resulting directional spreading of the spectrum may be larger.

In addition to the graphical results above, Tab. 5.6 provides the mean values of the errors obtained not only for beam and quartering wave conditions, but also for the other wave headings adopted in the tests. Again, the results stand for the estimations obtained adopting the variable damping model. The values provided in this table are the expected value of all the estimated mean relative errors computed for significant wave height and the mean wave period. In order to maintain the table suitable and manageable, the results were clustered in four different intervals, given by the corresponding theoretical peak periods of the wave spectra. Also, the absolute error of the estimated direction is included, in degrees. Finally, the relative errors are presented separately for JONSWAP and Torsethaugen spectra, to avoid the introduction of a bias in the groups.

Considering the whole range of wave periods tested, Tab. 5.6 attest that the maximum expected errors involved in the prediction of wave height and period are around $10 \%$ for JONSWAP seas, and $15 \%$ for the conditions corresponding to the bimodal Torsethaugen 


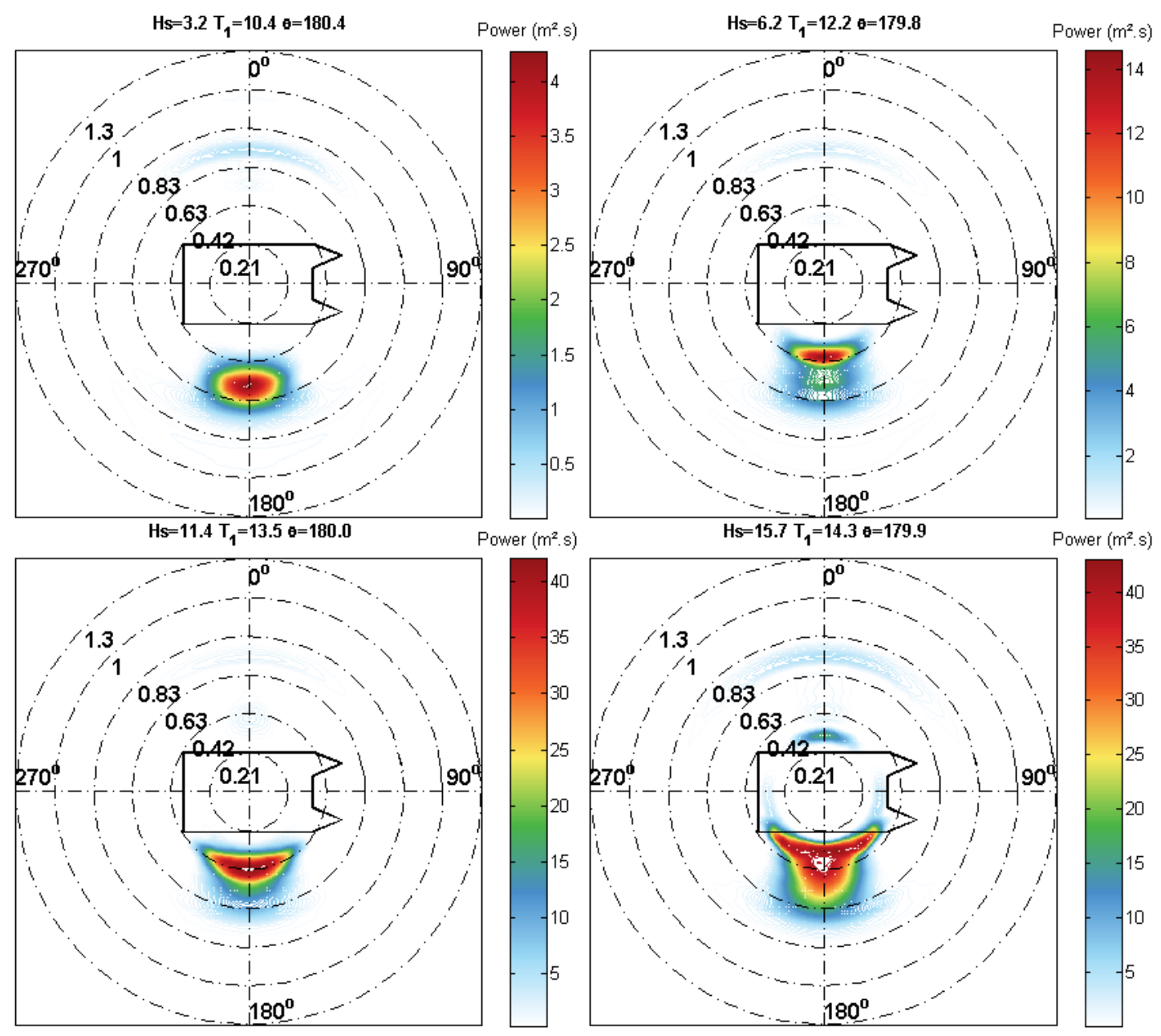

Figure 5.28: Wave spectra estimated using variable damping for the heading $90^{\circ}$, regarding the first realization of the sea conditions 2, 14, 25 and 32 .

spectra.

Regarding the shape of the wave spectrum, one may realize that the relative errors (for significant wave height and mean period) are amplified by the Torsethaugen model for all the wave headings. Again, this is not surprising since, compared to the JONSWAP model (see figure 3.1), the Torsethaugen spectrum places a larger amount of energy in the high frequency range, for which the dynamic response of the model is less pronounced.

Furthermore, the whole set of results show that the most unfavorable headings are those of bow $\left(180^{\circ}\right)$ and beam $\left(90^{\circ}\right)$ waves. Once again, this was expected because these are the situations when only 2 of the 3 motions used for the estimations are excited by the waves. On the other hand, the most favorable conditions are those closer to $135^{\circ}$ (or $45^{\circ}$ ), when all the 3 motions are excited and thus the amount of information available for the estimations is larger. These patterns are shown in Fig. 5.30, which presents the mean 


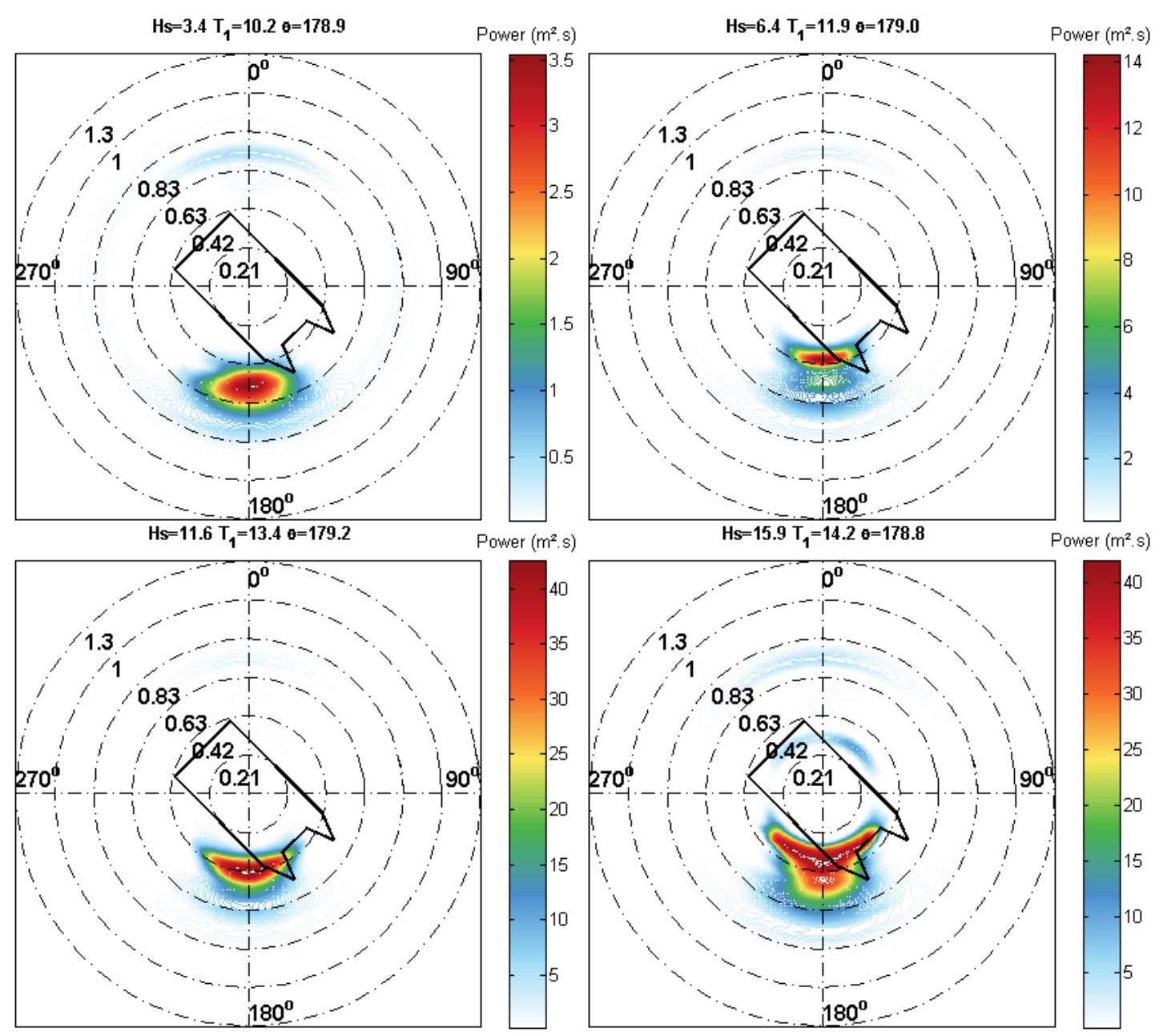

Figure 5.29: Wave spectra estimated using variable damping for the heading $135^{\circ}$, regarding the first realization.

of relative errors for $H s$ and $T_{p}$ (shown in previous table) in accordance with the heading condition. In this figure the data have been categorized in two different sets: blue markers, which stand for the error related to the sea conditions generated using the JONSWAP wave spectrum; and red markers, that represent the errors for the Torsethaugen wave conditions.

When the mean wave direction is concerned (see also, Fig. 5.28 and Fig. 5.29), although the estimated spectra present a somewhat larger spreading of energy if compared to those obtained from the wave probes, the prediction of the mean direction was very accurate for all the conditions tested. For instance, in all the conditions assessed during the experimental campaign it remained smaller than 3deg..

Finally, to conclude the discussion of the main aspects, some additional comments regarding the prediction of the most several conditions must be made. In fact, it is im- 
Table 5.6: Mean of the relative errors measured during the experimental campaign for $H_{s}$ and $T_{1}$, computed using the estimations obtained through the two realizations.

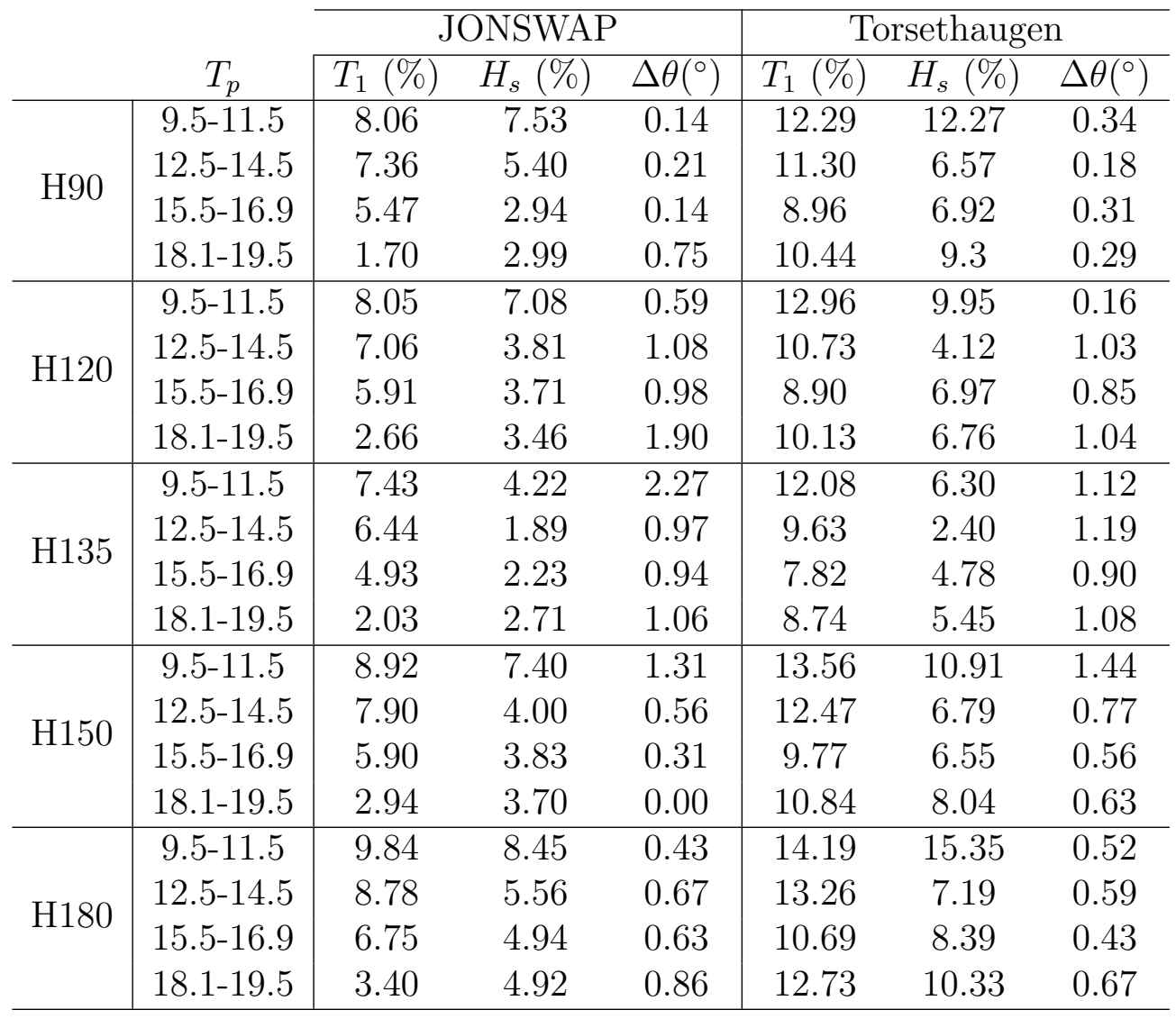
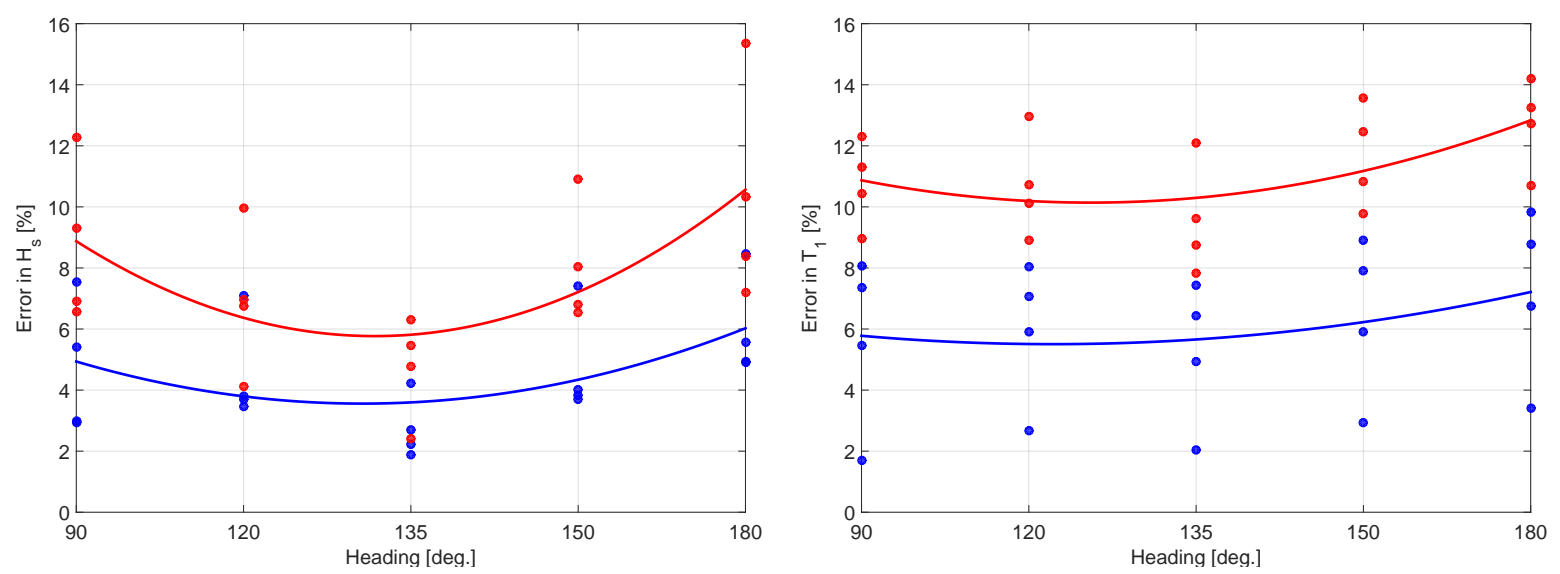

Figure 5.30: $H_{s}$ and $T_{p}$ mean relative errors from Tab. 5.6. Blue markers stand for the JONSWAP sea conditions and the red ones for those sea conditions generated by means of the Torsethaugen wave spectrum. The lines included in the plots stand as the quadratic fitting for each set of points (i.e. blue and red).

portant to realize that wave conditions with mean periods above to $12.7 \mathrm{~s}$, , that correspond to 100 years return period sea conditions of the Asgard field, could be captured by the VMB method with errors equal or lower than $10 \%$ in both wave height and period (for 
estimations obtained using the variable damping). These conditions involved waves with $H_{s}$ up to 16 meters and peak periods close to 20 seconds, which represent quite extreme waves. Obviously, one must remind that these good results were obtained in model-scale, in very controlled conditions. When considering the estimations made aboard a full-scale unit, other factors like short crested wave conditions as well as the presence of risers and mooring lines and the effects of wind and current on the motions, for example, will certainly impose a more demanding scenario for the predictions.

\subsubsection{Wave Inference using the alternative prior distribution}

In the following, a detailed comparison between the results obtained with the analysis presented above and those obtained with the alternative prior distribution (see section 4.4.2) is provided. Again, the set of RAOs adopted in this section was obtained with the variable damping model, which stands as the most appropriate damping approximation.

In order to provide an effective and proper visual formatting for the comparison of the results, a different arrangement is adopted: A representation of the distribution of the ratio between the value of the estimation and the measured value, for all the heading conditions (five), in terms of box-plots, plus the indication of $5 \%$ and $95 \%$ estimated percentile values. The plots in Figs. 5.31 to 5.36 report the following quantities, estimated using the different heading conditions, for the first realization ${ }^{9}$ of each sea condition tested:

- The lower and upper limit of each box indicate the $25 \%$ and $75 \%$ percentile levels, i.e. the lower and upper quartile respectively;

- The central mark indicates the $50 \%$ percentile level, i.e. the median;

- The bars in these plots indicate the the estimated $5 \%$ and $95 \%$ percentile levels respectively while the remaining data outside of the limits defined previously are likely outliers.;

Figures 5.31 to 5.33 show the results obtained by means of the conventional prior distribution, already discussed in the previous section. In this case, once more, only the

\footnotetext{
${ }^{9}$ In this case, since these figures aim at providing a comparison between the conventional and the alternative prior distribution, only the results regarding the first realization have been included.
} 


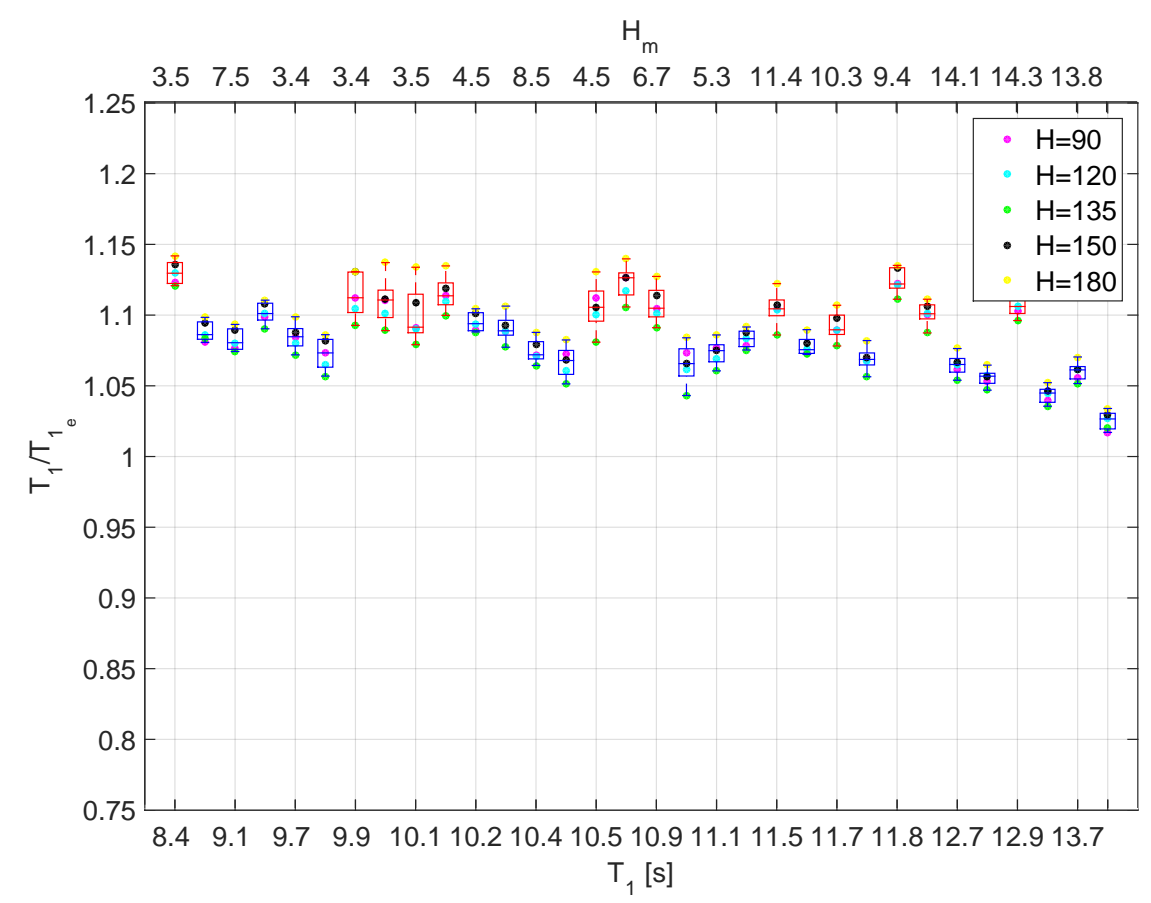

Figure 5.31: Box-plot showing the ratio between the estimated and measured mean wave period, obtained by means of the conventional approaches.

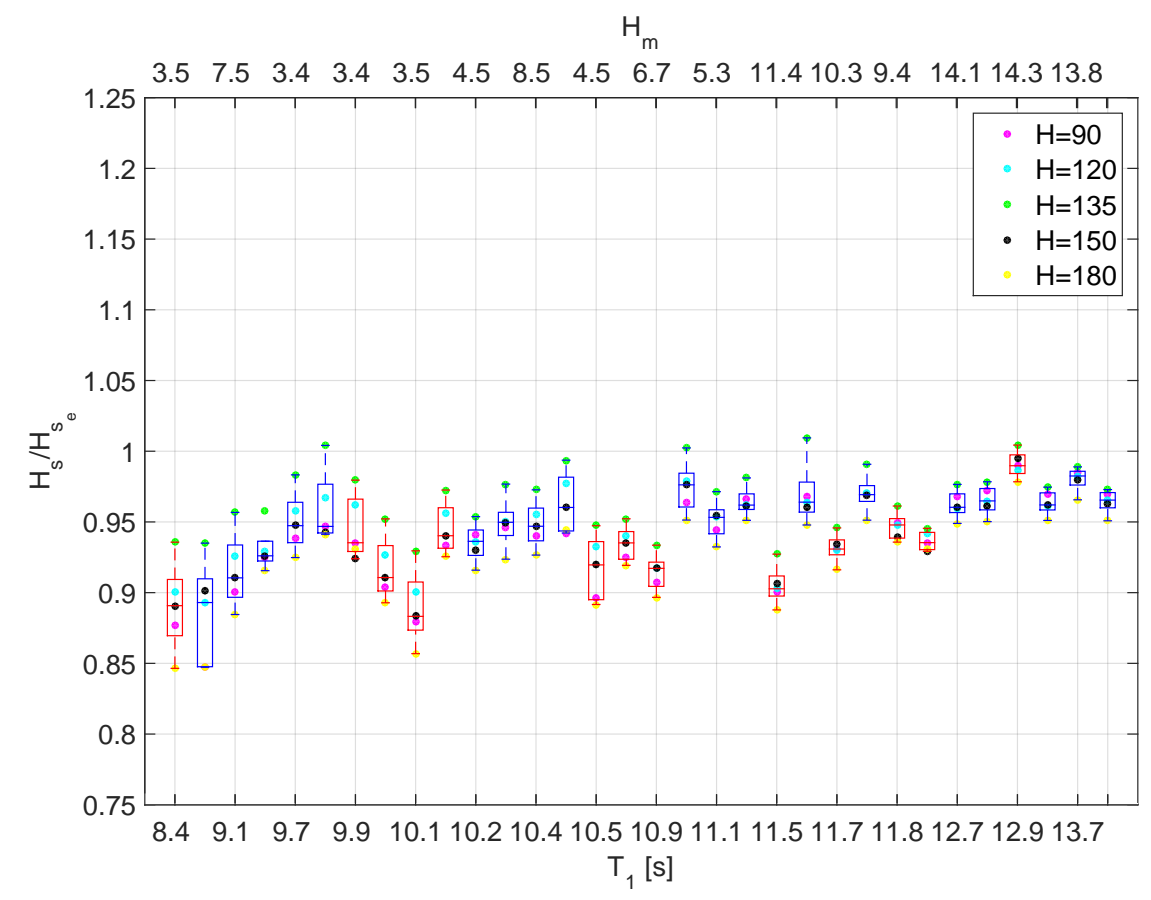

Figure 5.32: Box-plot showing the ratio between the estimated and measured significant wave height, obtained by means of the conventional approach.

results with the variable damping method are presented. These figures provide a general overview of the trends highlighted in section 5.4.3, according to the mean wave period. 


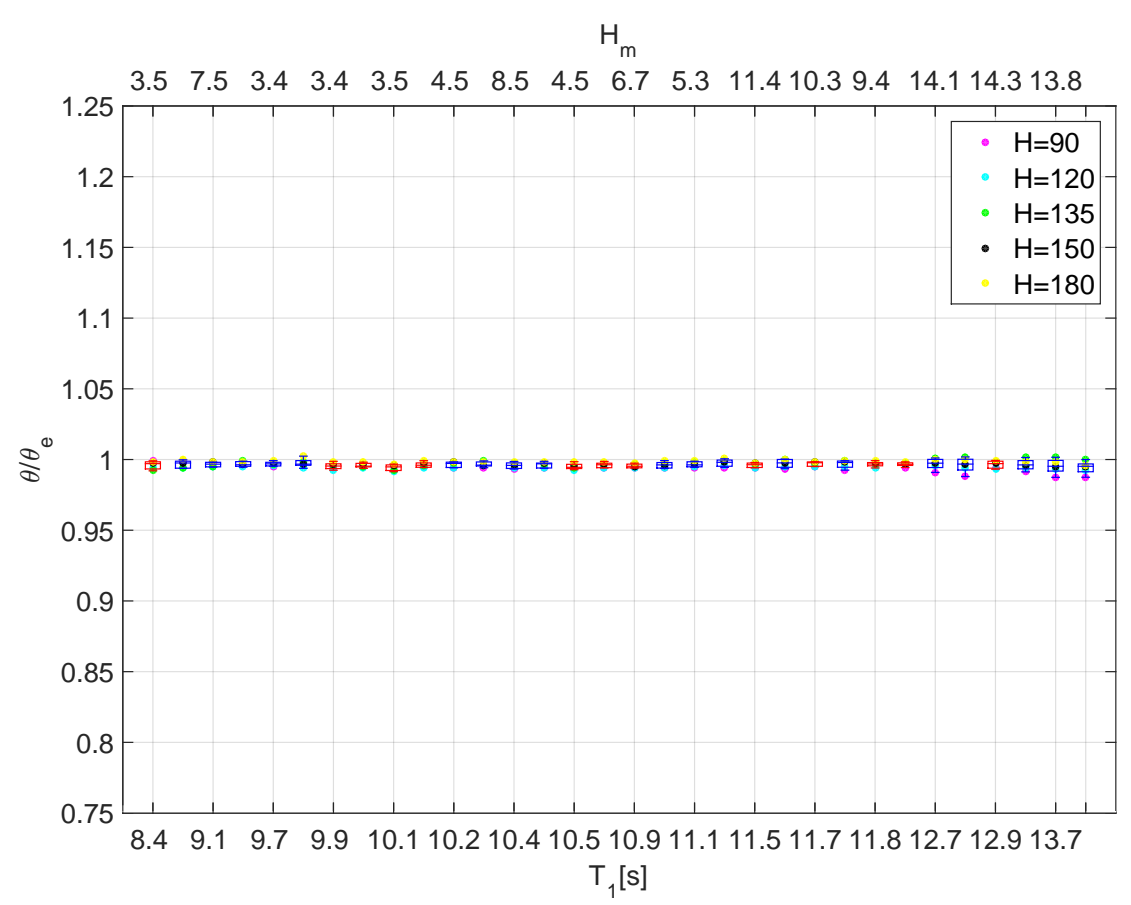

Figure 5.33: Box-plot showing the ratio between the estimated and measured mean wave direction, obtained by means of the conventional approach.

The box-plot representation of the data allows the reader to properly assess and quantify the variations imposed by the different heading conditions. Finally, it is interesting to point out that the differences between the estimations for different headings are reduced for sea conditions with large mean wave periods. This trend, that can be explained by the fact that in extreme sea conditions large amount of information is provided by the platform motions, is clearly shown in Figs. 5.31 and 5.32.

Similarly, Figs. 5.34 to 5.36 provide the results obtained using the alternative prior distribution, described in section 4.4.2. In this case, as it has been pointed out before, the set of RAOs has also been determined with the variable damping model. In general, the results obtained show a clear improvement of the estimations regarding the mean wave period (see Figs. 5.31 and 5.34, respectively), $T_{1}$. Furthermore, for calm to mild sea conditions the alternative prior distribution seems to result in a lower loss of energy. As a matter of fact, Fig. 5.35 shows a significant improvement on the estimation of the significant wave height for sea conditions characterized with low mean wave periods, if compared with the results obtained with the conventional prior distribution and presented in Fig. 5.32. 


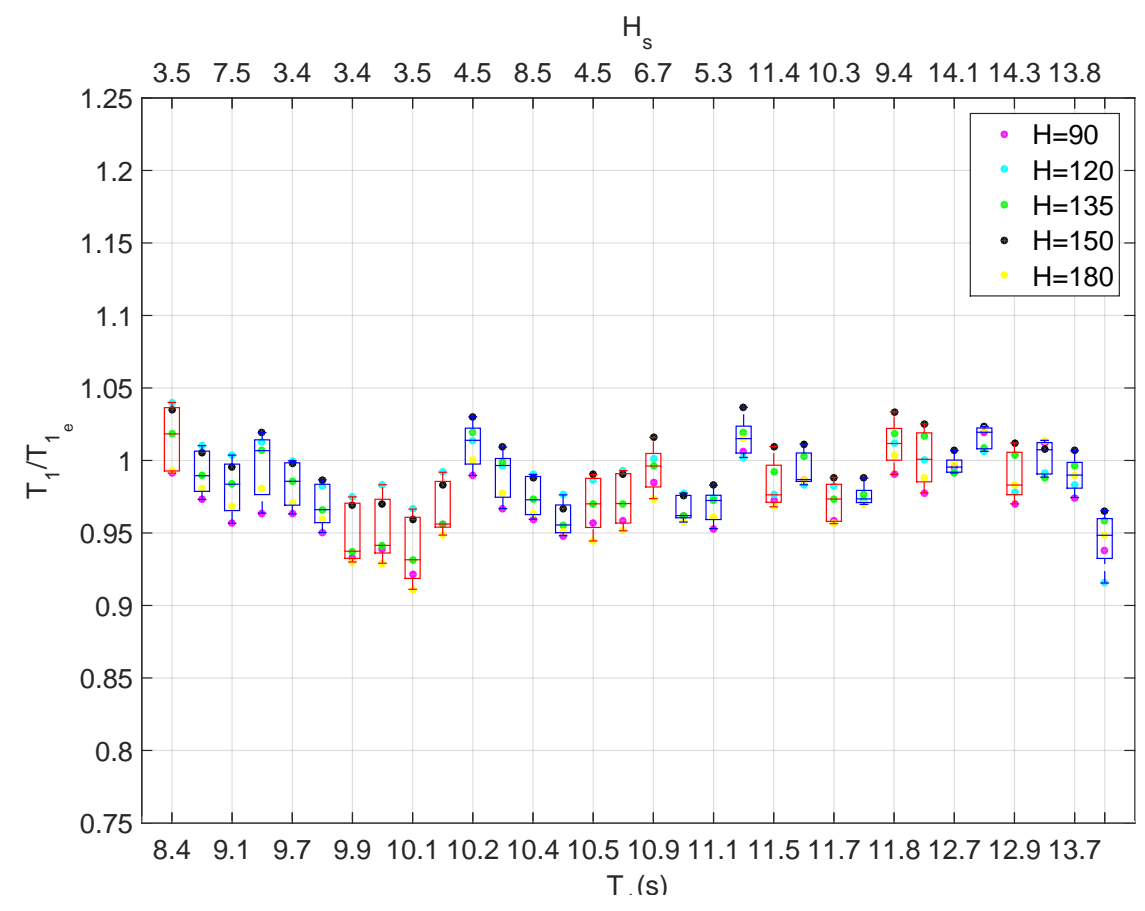

Figure 5.34: Box-plot showing the ratio between the estimated and measured mean wave period, obtained by means of the alternative prior distribution.

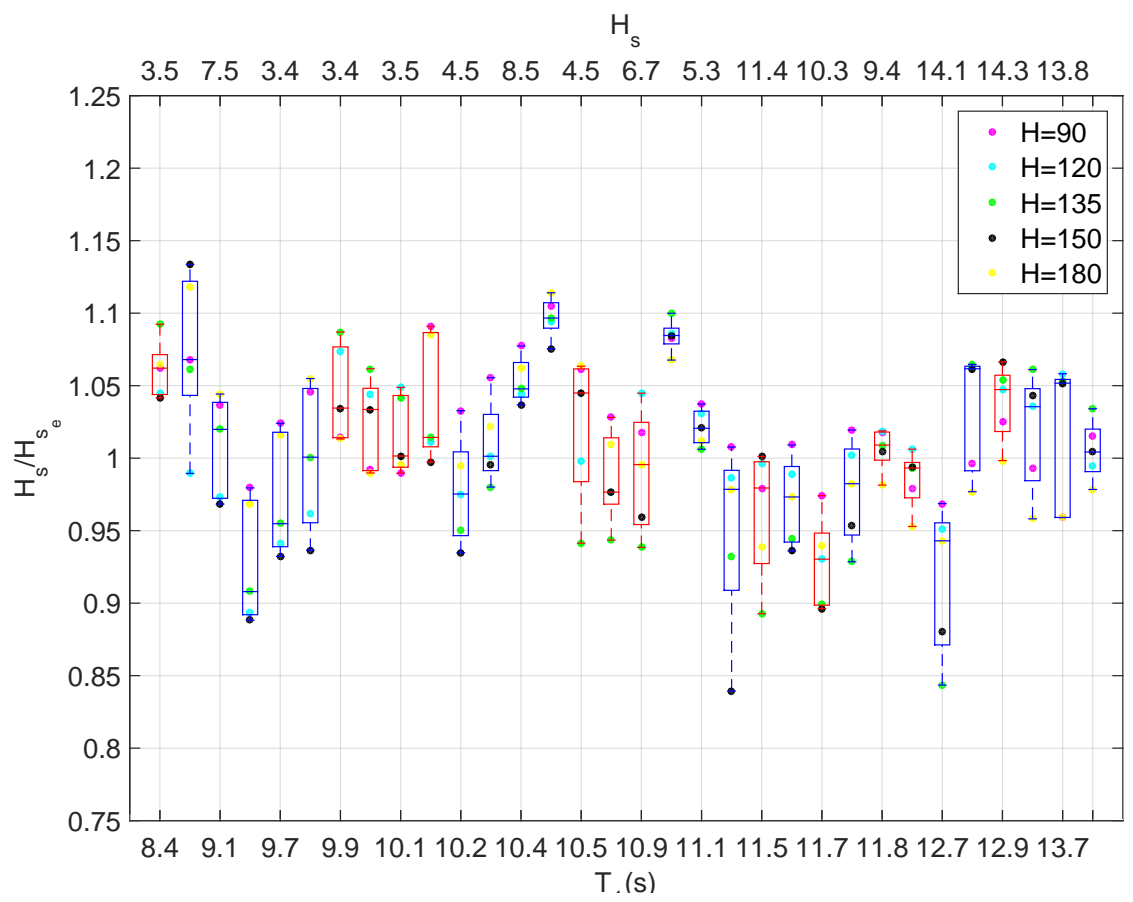

Figure 5.35: Box-plot showing the ratio between the estimated and measured significant wave height, obtained by means of the alternative prior distribution.

A clear disadvantage that arises from the use of the alternative prior distribution is that it tends to overestimate the energy of the wave spectrum (or the significant wave 


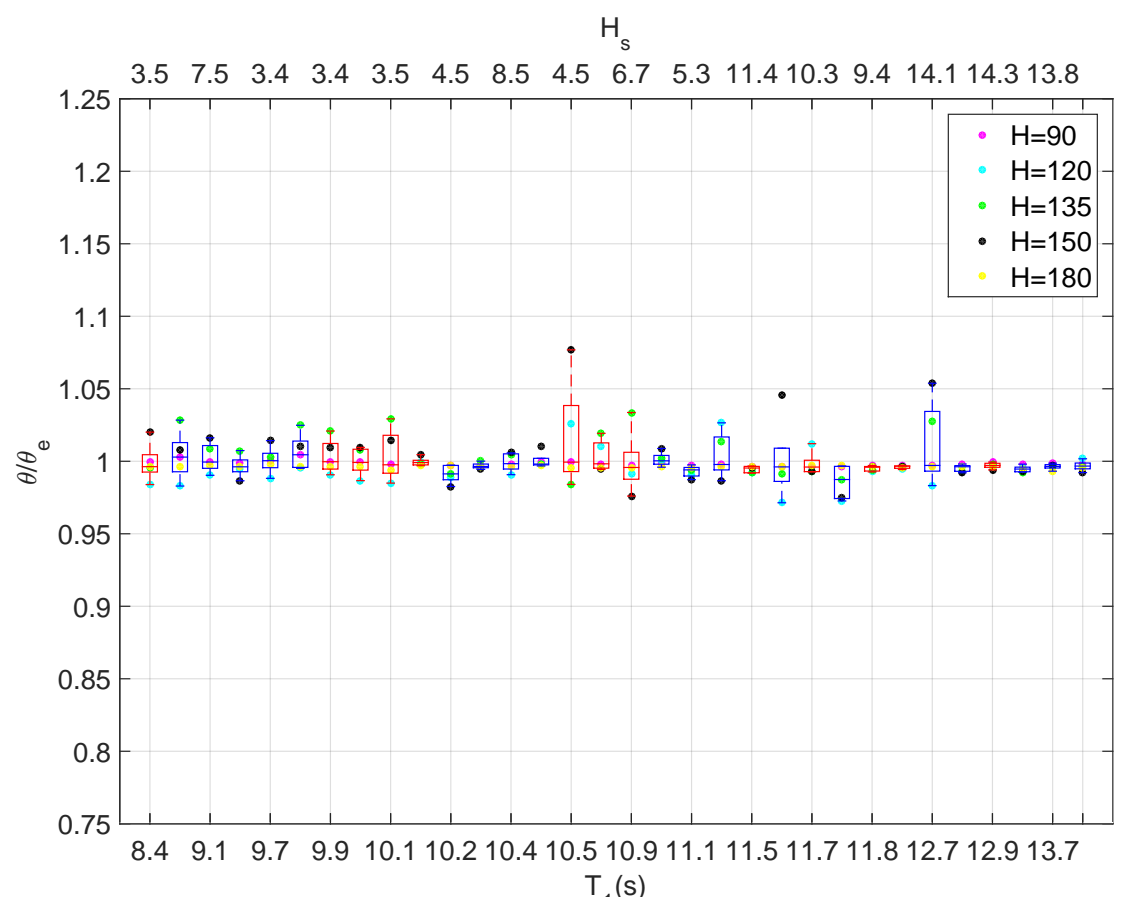

Figure 5.36: Box-plot showing the ratio between the estimated and measured mean wave direction, obtained by means of the alternative prior distribution.

height) in some cases. Also, the variability of the results for the same sea state obtained with different headings has been increased, leading to differences up to $18 \%$ between the estimations of $H_{s}$. In a similar way the estimations of the mean wave direction present the same pattern regarding the increment of variability of the estimation in accordance with the heading of the platform.

Figures 5.37 and 5.38 provide the energy spectrum in frequency-domain for different sea conditions tested. These figures include the theoretical spectrum (dashed black line), the measured spectrum (dashed green line) and the estimations obtained with the conventional and the alternative prior distribution, that correspond to the red and blue lines respectively. In general the results of the alternative prior distribution seems to provide estimations of the energy spectrum similar to the ones obtained through the alternative approach.

The influence of the cancellation points of the base of motions adopted to estimate the sea conditions may become meaningful for some sea conditions with large amounts of energy in the high frequency range (see for example sea condition 6, 12 and 15 in Fig. 5.37). Thus, resulting in some discrepancies with the measured wave spectra. For instance, the 
results of the alternative prior distribution are characterized by the overestimation of the energy close to periods of 9.2s. Regarding the low frequency range, although in one sea condition the energy peak is underestimated with the alternative approach (i.e. sea condition 12 in Fig. 5.37), the energy distribution for the remaining cases has been properly estimated.

In theory, the alternative prior distribution provides a mechanism that avoids the estimation of spurious energy in the frequency and direction range close to the cancellation points. The reliability of this approach has been assessed by the improvement of the estimations of the directional energy spreading for sea conditions with mean periods above to 12.7s. A detailed discussion about the estimations of the directional energy spectra is provided in the following (see Figs. 5.39 and 5.40). However, its effectiveness is limited by the fact that the heave, roll and pitch RAOs present several cancellation points in the high frequency range. It should be emphasized that although the alternative prior distribution aims at taking into account the uncertainties close to the zero points of the RAOs, large discrepancies between the measurements and the expected values of the motions may lead to the misleading estimation of the energy spectrum in certain frequency ranges linked to several cancellation points (from heave, roll and pitch).

Table 5.7 shows the mean value of the ratios presented in figures from 5.31 to 5.36. In this case the values are sorted according to the measured wave period of the first realization of the sea conditions. First, by inspecting the error statistics, it can be concluded that good agreement with the expected values is obtained in both cases. Nonetheless, as it has been pointed out before, the alternative prior distribution provides an improvement in the estimations obtained for the mean wave period. Regarding the estimations of the significant wave height and the mean wave direction, it can be stated that even though analogous results have been obtained in both cases, it is expected to obtain more accurate estimations with the alternative prior distribution for sea conditions with mean wave periods up to 11s.

Figures 5.39 and 5.40 show a comparison between the measured directional sea spectrum and the estimations, obtained by means of the two different approaches, the conventional approach and the Bayesian inference based on the alternative prior distribution. The examples selected comprise one moderate sea condition, one mild sea condition and 

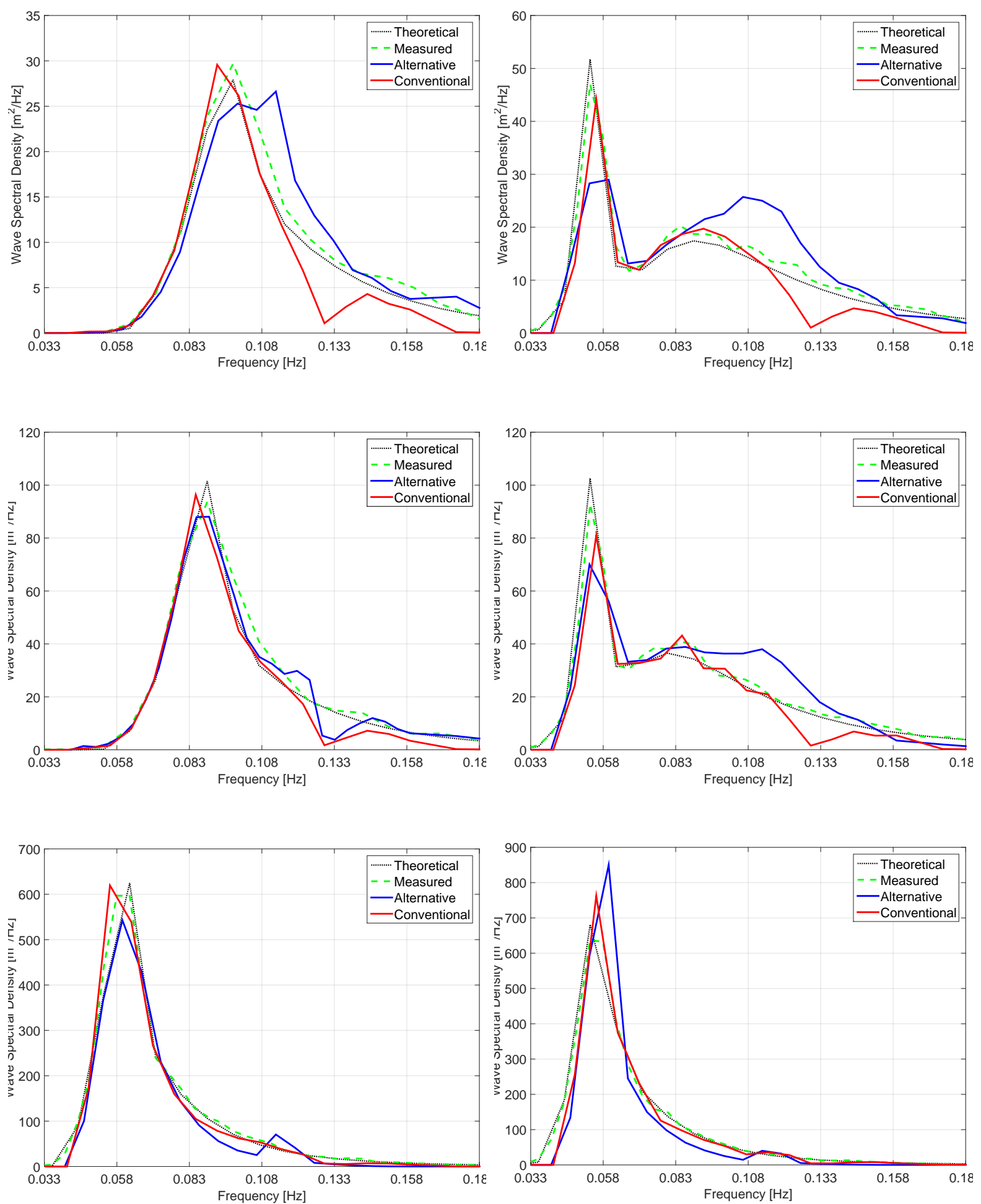

Figure 5.37: Expected energy distribution in frequency-domain for sea conditions: 6, 12, 15, 17, 30 and 31 (from left-to-right then top-to-bottom). For a heading equal to $90^{\circ}$.

two extreme sea conditions (see table 5.3). Fig. 5.39 provides some examples of the estimated directional wave spectrum obtained for beam sea conditions. In general, the results 

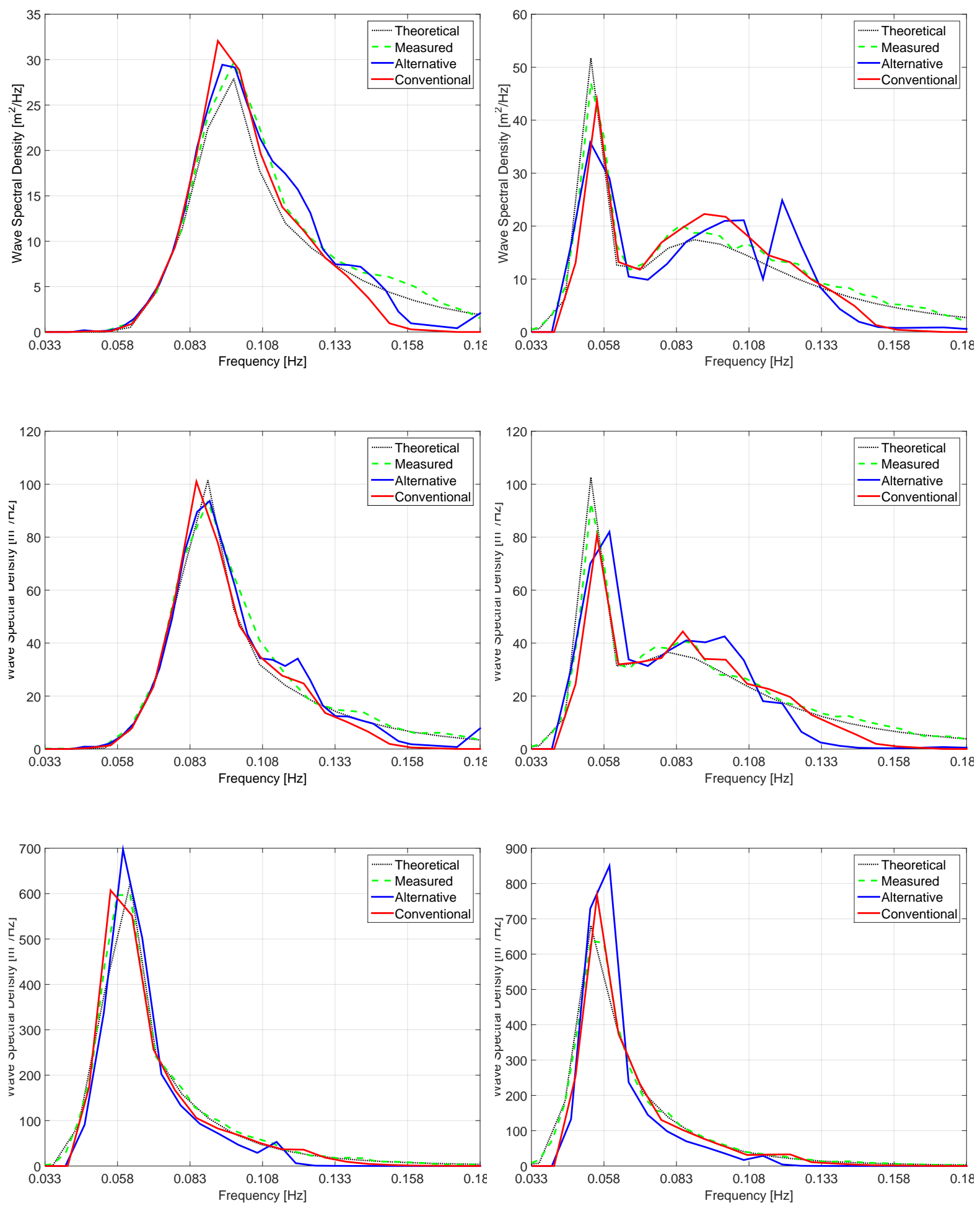

Figure 5.38: Expected energy distribution in frequency-domain for sea conditions: 6, 12, 15, 17, 30 and 31 (from left-to-right then top-to-bottom). For a heading equal to $135^{\circ}$.

obtained using the alternative prior distribution show good agreement with the directional sea spectrum computed by means of the Maximum Entropy Method. Nevertheless, the 
Table 5.7: Mean absolute values of the mean ratios provided in Figs. 5.31 to 5.36, organized according to the mean wave period.

\begin{tabular}{cccc|ccc} 
& \multicolumn{3}{c|}{ ALTERNATIVE } & \multicolumn{3}{c}{ CONVENTIONAL } \\
\cline { 2 - 6 }$T_{1}$ & $T_{1}(\%)$ & $H_{s}(\%)$ & $\Delta \theta\left(^{\circ}\right)$ & $T_{1}(\%)$ & $H_{s}(\%)$ & $\Delta \theta\left(^{\circ}\right)$ \\
\hline 8.41 & 1.02 & 1.06 & 0.67 & 1.13 & 0.90 & 1.02 \\
8.44 & 0.98 & 1.07 & 0.52 & 1.08 & 0.85 & 0.80 \\
9.07 & 0.98 & 1.02 & 0.09 & 1.08 & 0.93 & 0.52 \\
9.60 & 1.00 & 0.91 & 0.69 & 1.10 & 0.92 & 0.71 \\
9.65 & 0.98 & 0.95 & 0.07 & 1.09 & 0.94 & 0.74 \\
9.68 & 0.97 & 0.99 & 0.81 & 1.07 & 0.95 & 0.87 \\
9.96 & 0.94 & 1.03 & 0.05 & 1.10 & 0.36 & 0.58 \\
10.04 & 0.94 & 1.03 & 0.15 & 1.12 & 0.91 & 0.65 \\
10.12 & 0.93 & 0.99 & 0.43 & 1.10 & 0.88 & 0.93 \\
10.24 & 0.96 & 1.01 & 0.16 & 1.12 & 0.96 & 0.69 \\
10.25 & 1.01 & 0.98 & 1.56 & 1.10 & 0.94 & 0.66 \\
10.28 & 0.99 & 1.00 & 0.64 & 1.09 & 0.94 & 0.41 \\
10.35 & 0.97 & 1.05 & 0.30 & 1.08 & 0.94 & 0.68 \\
10.38 & 0.95 & 1.09 & 0.35 & 1.07 & 0.96 & 0.58 \\
10.49 & 0.97 & 1.04 & 0.11 & 1.11 & 0.94 & 0.66 \\
10.81 & 0.97 & 0.98 & 0.31 & 1.13 & 0.92 & 0.86 \\
10.88 & 0.99 & 0.99 & 0.77 & 1.11 & 0.92 & 0.38 \\
10.97 & 0.96 & 1.08 & 0.06 & 1.07 & 0.95 & 0.66 \\
11.06 & 0.97 & 1.02 & 0.07 & 1.06 & 0.95 & 0.50 \\
11.43 & 1.01 & 0.97 & 0.40 & 1.09 & 0.95 & 0.58 \\
11.46 & 0.97 & 0.98 & 0.84 & 1.11 & 0.91 & 0.46 \\
11.70 & 0.98 & 0.97 & 0.70 & 1.09 & 0.98 & 0.57 \\
11.73 & 0.97 & 0.93 & 0.72 & 1.09 & 0.93 & 0.36 \\
11.82 & 0.97 & 0.98 & 2.26 & 1.06 & 0.96 & 0.43 \\
11.84 & 1.01 & 1.00 & 0.74 & 1.11 & 0.95 & 0.61 \\
12.41 & 1.00 & 0.99 & 0.61 & 1.10 & 0.94 & 0.52 \\
12.70 & 0.99 & 0.94 & 0.52 & 1.06 & 0.97 & 0.68 \\
12.91 & 1.01 & 1.06 & 0.68 & 1.06 & 0.97 & 0.85 \\
12.94 & 0.98 & 1.05 & 0.52 & 1.10 & 0.99 & 0.97 \\
13.65 & 1.00 & 1.04 & 0.97 & 1.04 & 0.97 & 0.86 \\
13.73 & 0.98 & 1.05 & 0.64 & 1.06 & 0.98 & 0.70 \\
14.70 & 0.94 & 1.00 & 0.60 & 1.01 & 0.97 & 0.51 \\
\hline & & & & & &
\end{tabular}

same approach leads to a non-smooth spectrum pattern for those quartering sea conditions with small wave periods (for instance, see Fig. 5.39). This can be linked to the fact that the alternative prior distribution no longer imposes the smoothness conditions as a necessary requirement of the solution, something that also leads to minor misleading estimation of the energy distribution in the direction. In any case, this drawback does not affect the energy distribution in the frequency-domain (see Figs. 5.37 and 5.38). Once again, it is important to remember that the long crested waves stand as a limiting condi- 
tion for the conventional approach, since it assumes an smooth distribution in the energy domain. Therefore, one may realize the relevance of the assessment regarding the performance of the methodologies adopted in this work by means of sea conditions generated (or measured) with a certain degree of spreading, something that has been left for future work.

By inspecting these figures it can be concluded that the alternative approach, in general, provides solutions with narrow spread of the energy in the direction. However, the use of the smoothness only as a weak condition, in the alternative prior distribution, may generate non-smooth solutions quartering seas. Another trend that can be identified is that spreading of the solutions obtained by means of both approaches are larger for extreme sea states than mild/calm sea states. This is due to the influence of the zero point that the heave RAO presents close to $22 \mathrm{~s}$. It is worth to remember that the alternative approach provides estimations characterized by a smaller spreading than the ones obtained through the conventional approach.

To sum up, it can be concluded that the alternative prior distribution brings some improvements if compared with the conventional approach. For instance, the alternative approach seems to provide more accurate estimations of sea conditions characterized by low mean wave periods. Similarly, the spreading of the energy in direction is narrower when the sea state is estimated by means of the alternative approach than if it is inferred using the conventional prior distribution. Nonetheless, the variance of the results, with regards to the different heading conditions, is increased when they are estimated though the alternative prior distribution.

Finally, another advantage obtained with the alternative prior distribution is the reduction of the computing time. As it has been pointed before, the computations were carried out with a desktop personal computer using MATLAB ${ }^{\circledR} 2014 \mathrm{a}$. In average, the software took $28 \mathrm{~s}$. to estimate one sea condition using the conventional approach, without taking into account the time spent during pre-calibration procedure of the hyperparameters. This mean time is reduced to only $6.7 \mathrm{~s}$ when the alternative prior distribution is adopted. This may be relevant for future applications of the method, such as its use to obtain a preliminary estimation of the sea state or even when large data sets are analyzed. 


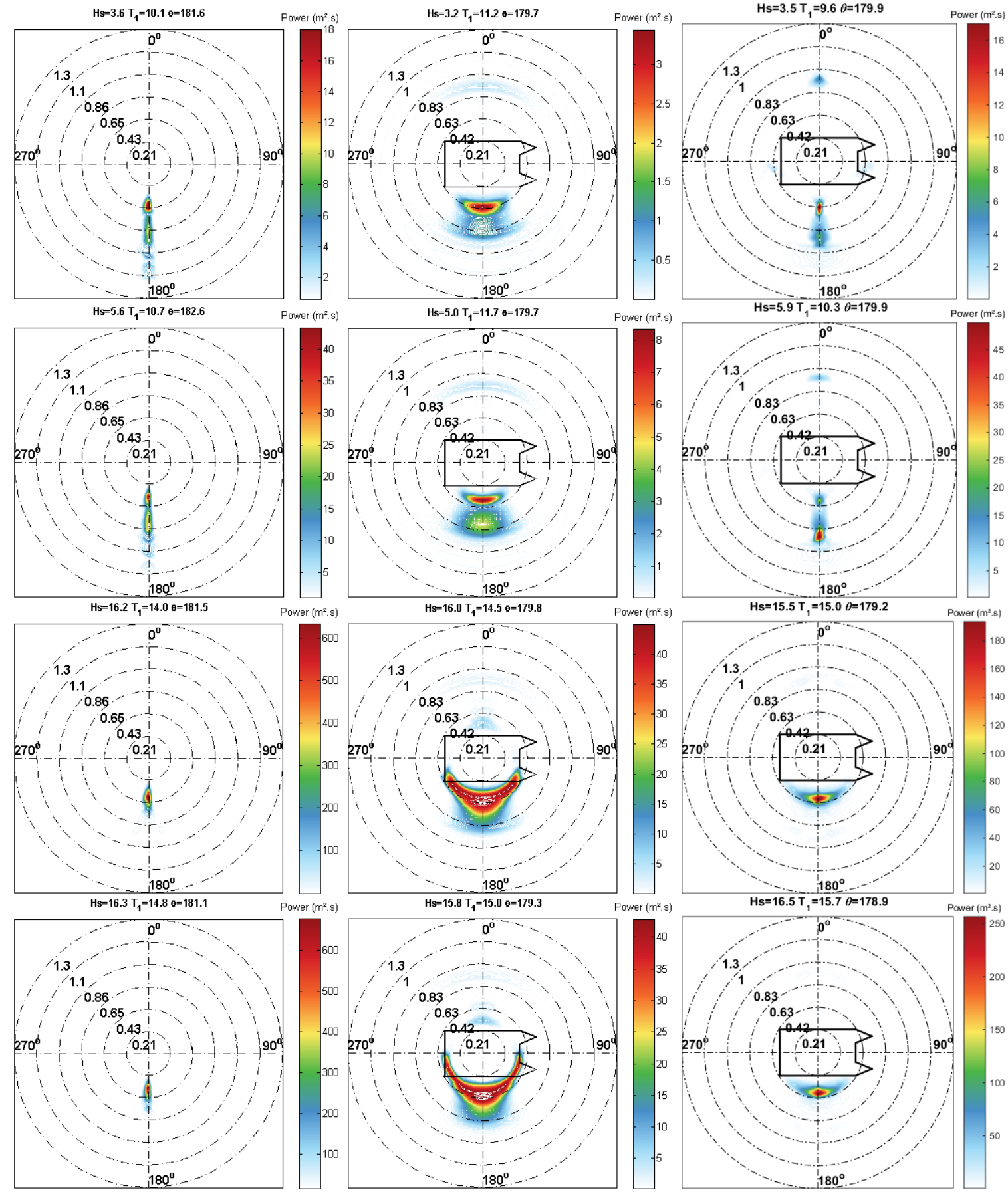

Figure 5.39: Comparison of directional wave spectra estimated with three different methods (for a heading equal to 90deg.). From left-to-right: Measured sea spectrum, the ones estimated with the conventional prior distribution and the output obtained with the alternative approach. From top-to-bottom: Sea conditions 4, 12, 30 and 31. 


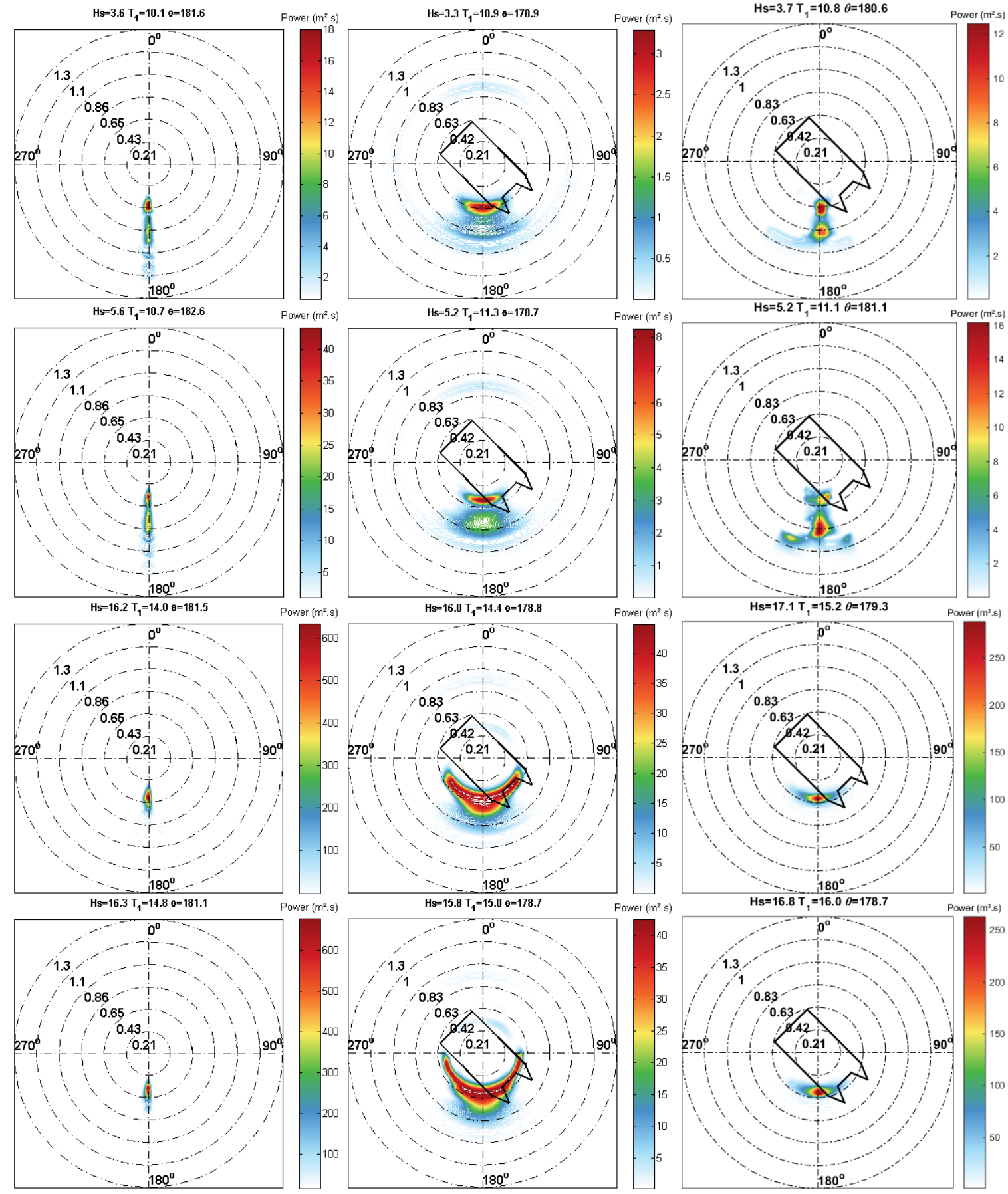

Figure 5.40: Directional wave spectra for a platform heading equal to 135deg., estimated using three different methods. From left-to-right: Measured sea spectrum, the ones estimated with the conventional prior distribution and the output obtained with the alternative approach. From top-to-bottom: Sea conditions 4, 12, 30 and 31. 


\section{CONCLUSIONS AND FUTURE WORK}

This thesis has primarily assessed the usefulness of employing a semisubmersible platform for VMB estimations of local sea spectra. The analysis performed was based on the results obtained through an extensive model-scale experimental campaign carried out at the USP's wave basin (TPN-USP), using a (1 : 120) scale model.

In accordance with the objectives of this thesis, the following tasks have been fulfilled and subsequent conclusions drawn:

1. A systematical analysis of the dynamics of the semisubmersible platform has been carried out, including a detailed assessment regarding the main hypothesis adopted to model the sea wave conditions as well as to linearize the platform wave responses. Moreover, the VMB Bayesian wave inference with three hyperparameters, as proposed in (SIMOS et al., 2012), has been adopted in order to evaluate the reliability of the platform as a VMB wave sensor.

- The dynamics of the motions of the platform has been analyzed numerically in time-domain and in frequency-domain, showing that the platform presents good (linear) response characteristics for being used as a wave sensor, particularly for periods below 20s.

- Since the sea conditions tested at the wave basin range from calm/mild conditions to 100yr-return events, they allowed a comprehensive assessment of the semisubmersible platform as a VMB wave sensor in calm and extreme sea states.

- The analysis carried out by means of the coherence functions has shown that the heave-roll-pitch basis is the most appropriate for inferring the sea states using the semisubmersible platform. 
- Results obtained adopting this approach revealed that all the wave conditions tested could be accurately inferred from the Bayesian method, keeping the errors within reasonable limits. On the one hand, the errors were larger in the most unfavorable conditions, which correspond to the ones with small motions (small periods or mild seas) and in the cases when the sea only excited two of the three motions (bow and beam waves). Thus, the wave height and the mean wave period were estimated with errors always below $18 \%$ and $15 \%$ for the most unfavorable heading conditions, respectively. Regarding the mean wave direction the results shown that it can be estimated with an error lower than $3 \mathrm{deg}$.

- The errors are reduced in the cases were the platform presents larger motions, or the existing ones provide more information, which are given when the heading is close to $135^{\circ}$ and for the more severe wave conditions (when the wave induce more pronounced motions). The errors for the most favorable conditions are $0.05 \%, 1.3 \%$ and $0.6 \mathrm{deg}$., for the estimations concerning the significant wave height, mean wave period and mean direction, respectively. All the results confirmed the expected biases of overestimating the wave period while underestimating the wave height, deviations that are progressively smaller as the wave period increases and leading to better estimation regarding the extreme sea conditions.

2. A detailed discussion regarding the prior distribution introduced by different authors has been provided. The main differences between them and how they are related to each other have been also addressed in the analysis provided. The conclusions drawn are:

- The Bayesian learning methodology adopted, see (TANNURI et al., 2003), to assess the reliability of using the measured motions of the platform to estimate sea conditions, has provided results that show good agreement with the measurements obtained by means of conventional waves probes. However, the semisubmersible platform was tested under carefully controlled experimental conditions, reducing the uncertainties of the linear model. In fact, other works developed using the same inference technique, based on the measured motions 
of a FPSO, e.g. (BISPO, 2011a), have pointed out misleading estimations under certain sea conditions.

- In principle, the inference results can be improved by means of simple modifications of the prior distributions adopted. In fact, an alternative prior distribution has been developed, aiming to account for the possible drawbacks of the current approach.

- The comparison between the outputs obtained with the conventional approach (TANNURI et al., 2003) and the ones resulting from the inference method based on the alternative prior distribution has shown that the adoption of the later may lead to significant improvements. In fact, the error associated with the estimation of the mean wave period has been reduced in general by a factor equal to $5 \%$. Similarly, the estimation of the wave height revealed an improvement of the order of $3 \%$ regarding the mean error of the estimations, although it presents an increment of the error variability concerning different headings.

- An aspect of the alternative prior distribution that needs to be reviewed is that for certain heading conditions $\left(120^{\circ}, 135^{\circ}\right.$ and $\left.150^{\circ}\right)$, the estimations result in non-smooth patterns of the sea spectra. Concerning the relative errors of the estimations of the statistical parameters of the sea, it can be stated that they remained almost unchanged. The resulting unsmoothed pattern of the sea spectrum for these headings may be related to the fact that the smoothness conditions, adopted in the alternative prior distribution, act only as a weak restriction.

3. An assessment regarding the non-linearities close to the heave resonance has been carried out by means of numerical analysis in frequency-domain and time-domain.

- Non-linear heave responses were identified close the resonance frequency, that is within the frequency range of interest. Regarding the roll and pitch motions, their resonances are equal to $74 \mathrm{~s}$ and $88 \mathrm{~s}$. Therefore, for the purposes of this work the platform is expected to respond linearly in roll and pitch.

- It is already known that the main source of non-linearities close to the resonances arise from drag effects. As a matter of fact, the viscous damping of the 
heave motions is mainly governed by separation effects of the pontoons.

- In order to properly model the responses of the heave motion, a simple linear damping model has been proposed to take account of the non-liner resonant responses, which indeed showed a good agreement with the decay tests carried out in still water.

4. The results for more severe sea states are promising, even when the RAOs are modelled in a conventional linear analysis in frequency-domain. Furthermore, the adoption of the alternative prior distribution seems to provide promising improvements of the estimations of the sea conditions. Also, this prior distribution does not need previous calibration of the hyperparameters (avoiding the use of numerical simulations or a dedicated experimental campaign). This property, along with the fact that the computational time is reduced, leads to a less time-consuming methodology.

- It may perhaps be observed that the results with the RAOs obtained from different approaches also highlighted the importance of a proper modelling of the transfer functions of motion.

- In the experimental setup, a soft mooring system was used and the major source of drag was the platform itself. In this condition, the results obtained when using the variable external damping method (a damping ratio proportional to the recorded heave amplitude in each test) proved to be a good option for dealing with effects of the non-linear viscous damping.

- Other possible effects due to the environmental conditions, such as wind and currents, or the submerged lines, must be properly assessed by means of a dedicated experimental campaign.

- Another characteristic that may have a relevant influence on the accuracy of the estimations is the spreading of the sea. During experimental campaign only long crested sea conditions were tested, which provides a preliminary assessment of the reliability of the semisubmersible as a VMB wave inference sensor. Long crested seas, however, stand as a limiting case for the conventional approach and the use of sea states with spreading in direction may result in the improvement of the estimations. 
- The results obtained also attest that the semisubmersible platforms, although it is characterized by small motion amplitudes, it responds linearly in a wide range of sea conditions. Furthermore, it is known that in the operating condition these platforms does not present variations of their metacentric height and draft, which facilitates the estimations of the most appropriate set of RAOs used to infer the sea conditions.

As a general conclusion, one may state that the results obtained with the small-scale tests indicate that semisubmersible platform indeed may be considered as a good choice for the VMB wave inference. Finally, since the semisubmersible platform can provide reliable account of the sea conditions that imposed the measurement motions, in wide range of frequencies, they can be adopted to complement the wave buoys network installed along the Brazilian coast. Figure 6.1 presents the resulting wave monitoring network if Fig. 1.5 is updated including only the semisubmersible platforms operated by Petrobras (in dark blue), illustrating the improvement of the monitoring network.

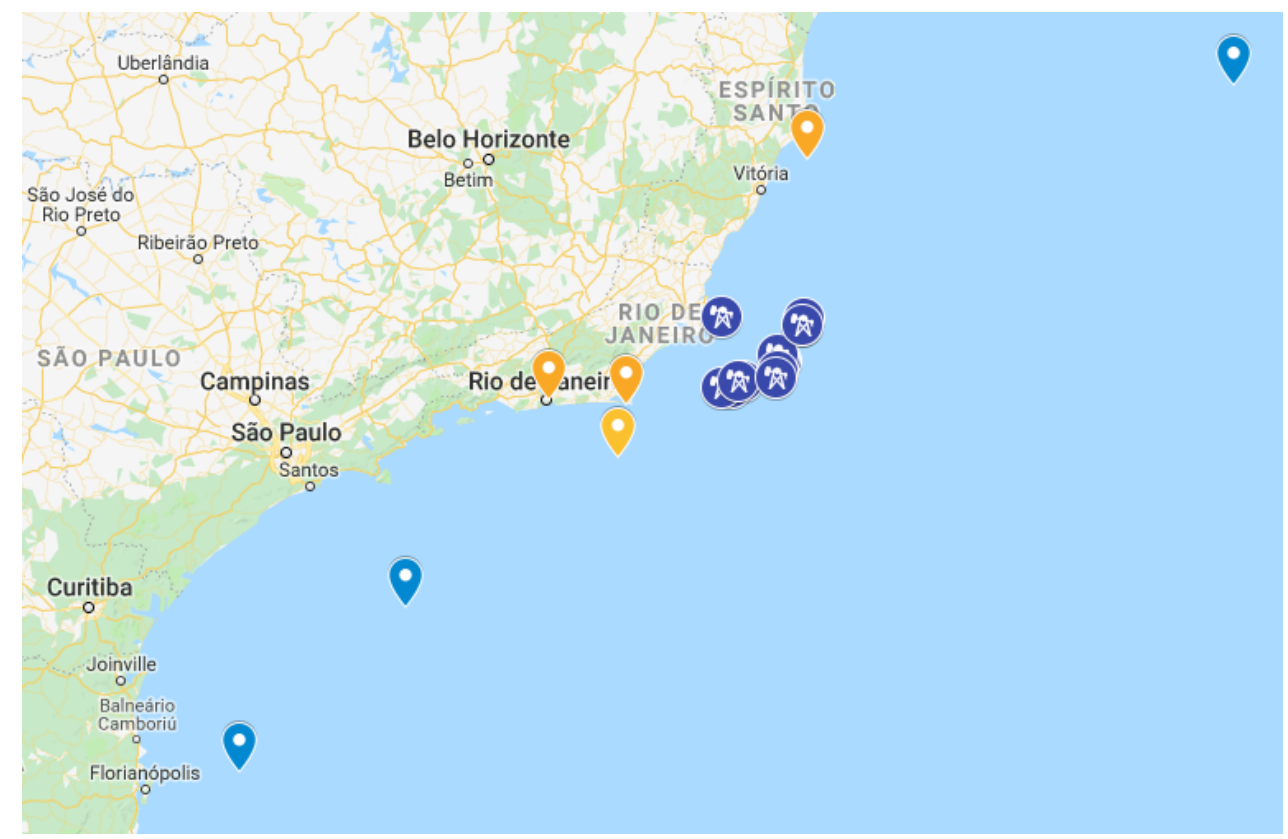

Figure 6.1: Wave buoys and semisubmersible offshore paltforms stations in the Santos, Campos and Espíto Santo basins. Information from (GOOS-BRASIL, 2018), (NOAA, 2018) and (PETROBRAS, 2018). 


\subsection{Major findings}

This thesis aims at researching the VMB wave inference method using the motions of a semisubmersible platform. The major contributions obtained during the development of this work can be summarized as follows:

- When estimating the wave conditions based on the measured motions of a smallscale model of a semisubmersible platform, the results obtained show that semisubmersible platforms may be considered as a good choice for the VMB wave inference. In fact, the hydrodynamic characteristics of the platform (high natural periods) increase the reliability of the model adopted. Therefore, for large semisubmersible platforms, the interval of estimation may be more restricted in the high frequency range. Generally, the accuracy of the estimations is improved as the wave period increases, which is a benefit to the semisubmersible as a VMB wave sensor considering the more severe wave scenarios expected where these platforms are usually developed.

- Non-linear effects should be included in the excitation-response model of the platform to increase the accuracy of the estimations, especially in sea conditions characterized by large peaks periods that have energy close to the heave resonance. From the Morison quadratic drag equation a linear damping estimation has been proposed. This methodology aims to provide a simple approach to estimate the non-linear drag effects acting on the heave motion of a semisubmersible platform. The estimations obtained through this model show good agreement with the experimental data from the heave decay tests.

- The Bayesian VMB wave inference method as adopted by Tannuri et al. (2003) provided good estimations when compared with the experimental measurements carried out at the wave basin by means of wave probes. However, this inference model must be pre-calibrated, procedure that may involve numerical simulations as well as experimental measurements. Furthermore, in some specific conditions previous works have identified some limitations. In order to mitigate these shortcomings, an alternative prior distribution is provided in this thesis. The results obtained through this prior distribution are in agreement with the experimental measurements and in 
some frequency ranges outperform the estimations obtained by means of the conventional approach. Another important advantage obtained with this alternative approach is the reduction of the computing time to estimate the sea conditions.

Summing up, the main contributions of this thesis are the assessment of the use of a large semisubmersible platform as a VMB wave sensor and the development of an alternative prior distribution. It is shown that the prior knowledge of the variance of each element of the unknown spectrum can be estimated through the measured motions. An extensive analysis of analytical, numerical and small-scale experimental data has been carried out. Finally, this thesis also provides a systematic discussion regarding the characteristics of the sea states generated at the wave basin (Gaussianity, Stationarity and the optimal run length) as well as the boundary conditions adopted in the Bayesian approach to estimate the sea states.

\subsection{Future work}

Naturally, a substantial amount of possible future work threads arise from the topics discussed in this thesis. From these, the following tasks are considered the more relevant to properly addressing during future stages of this research:

- The relevance of a more refined experimental analysis of the effects the moorings and risers may have on the motions of the unit is envisaged, and the same is true regarding the action of wind and current. Furthermore, the use of the full-scale data to make wave inference will be useful to understand the real effects that the moorings lines and risers may have on the motions of the semisubmersible platform.

- Regarding the use of full-scale data, it may also be useful to fully assess the reliability of the semisubmersible platform as a VMB wave inference wave sensor. The results obtained through the experimental campaign carried out in the wave basin attested that the method was capable of capturing very well all the wave conditions tested. However, full-scale data may bring challenges that are different to the ones that were overcome in this thesis.

Following the procedure adopted in (BISPO, 2011b) to validate the use of a FPSO as VMB wave sensor, it can be advised to adopt a minimum time span equal to 
24 months of full-scale data. This approach would allow to take into consideration the seasonal variability of the sea conditions (including $H_{s}, T_{p}$ and $\theta$ ) when the assessment of the use of a semisubmersible platform is carried out.

- The Åsgard-B semisubmersible platform is characterized by large resonant periods for the roll and pitch motions. However, this is not the general standard for the all semisubmersible platform, which usually present lower natural periods for pitch and roll motions (slightly above to $30 \mathrm{~s}$ ). Since one the objectives is the expansion of the wave buoys monitoring network in the Brazilian coast, it is important to carry out some tests with geometries for the semisubmersible platform operated by Petrobras, such as P-18, P-20, P-51 and P-52.

- Regarding the alternative prior distribution, there are still some problems with the smoothness of the outputs. In fact, for bow sea conditions the estimated directional wave spectrum in some cases is characterized by a noisy pattern. The adoption of smoothing functions, based on the estimated energy distribution, may provide a suitable solution for this shortcoming.

- It has been shown that the uncertainty introduced by the cancellation points of the RAOs may generate misleading estimations of the sea conditions. The alternative prior distribution proposed in this work provides a proper mechanism to avoid spurious energy estimations near the cancellation points. However, it would be useful to properly study if the linear approach adopted to estimate the prior distribution (see Eq. 4.40) is the one that leads the best estimations.

- The boundary conditions adopted to compute the prior distribution have been discussed in appendix E. Moreover, it was shown that some of these conditions can be modified to mitigate some drawbacks identified by other authors when a FPSO is adopted as a VMB wave sensor (see Bispo et al. (2016)), such as the tendency of the method of locating spurious energy peaks at the bow and stern of the vessel in beam sea conditions. Due to the fact that the estimations obtained during this research do not show the limitations identified by Bispo et al. (2016), an assessment regarding the possible improvements provided by the changes proposed for the prior distribution has been left as a future work. 
- This thesis is based on an extensive analysis of the results obtained using two different approaches for the Bayesian inference. In both cases good agreement with the measurements is obtained, showing that a semisubmersible platform can be used as a proper VMB wave sensor. However, it would be interesting to determine the statistical significance of these results, or in other words, what are the chances of obtaining the same results if a similar model-scale experimental campaign is carried out again? The most common approach adopted to answer this question is to generate large amounts of data through an experimental campaign. However, this approach is likely to result in an expensive research campaign and in some cases the results drawn may be inconclusive. In fact, large amounts of data tend to normalize biases, specially when they do not properly represent the majority of the population of interest (in this case the semisubmersible platforms). For these reasons, it is proposed as a future work the evaluation of the statistical significance of the results by means of alternative statistical techniques, such as the Bayesian factors. By adopting this approach, the main challenge to be faced would be to properly estimate the marginal distribution of measuring the data obtained from the measured motions of the platform, $P(B)$.

- Finally, another line of future work is the use of semisubmersible wind turbine platforms as a base for the VMB wave inference. As a matter of fact, these platforms stand a popular technology as an offshore wind turbine foundation and they usually are carefully monitored. Although the dynamics of these platforms is similar to the one presented by conventional O\&G conventional semisubmersible platforms, a dedicated experimental campaign is needed to properly assess the effects that the aerodynamic wind forces may have on the platform motions. 


\section{REFERENCES}

AKAIKE, H. Likelihood and the bayes procedure. Trabajos de estadística y de investigación operativa, Springer, v. 31, n. 1, p. 143-166, 1980.

AKAIKE, H. Information theory and an extension of the maximum likelihood principle. In: Breakthroughs in statistics. New York, NY.: Springer, 1992. p. 610-624.

ALLENDER, J.; AUDUNSON, T.; BARSTOW, S.; BJERKEN, S.; KROGSTAD, H.; STEINBAKKE, P.; VARTDAL, L.; BORGMAN, L.; GRAHAM, C. The wadic project: a comprehensive field evaluation of directional wave instrumentation. Ocean engineering, Elsevier, v. 16, n. 5, p. 505-536, 1989.

ANSYS, A. Aqwa theory manual. ANSYS, Inc, 2013.

ÅSTRÖM, K. J. Introduction to stochastic control theory. London, UK.: Courier Corporation, 2012.

BARBER, N. F.; URSELL, F. The generation and propagation of ocean waves and swell. i. wave periods and velocities. Phil. Trans. R. Soc. Lond. A, The Royal Society, v. 240, n. 824 , p. 527-560, 1948.

BARSTOW, S. F.; BIDLOT, J.-R.; CAIRES, S.; DONELAN, M. A.; DRENNAN, W. M.; DUPUIS, H.; GRABER, H. C.; GREEN, J. J.; GRONLIE, O.; GUÉRIN, C. et al. Measuring and analysing the directional spectrum of ocean waves. Luxembourg, LU.: Office for Official Publications of the European Communities, 2005.

BENDAT, J.; PIERSOL, A. Random Data: Analysis and Measurement Procedures. Hoboken, NJ.: Wiley, 2010. (Wiley Series in Probability and Statistics).

BENKE, K. K.; LOWELL, K. E.; HAMILTON, A. J. Parameter uncertainty, sensitivity analysis and prediction error in a water-balance hydrological model. Mathematical and Computer Modelling, Elsevier, v. 47, n. 11, p. 1134-1149, 2008.

BERNARDO, J.; SMITH, A. Bayesian Theory. New York, NY.: John Wiley \& Sons Canada, Limited, 2006. (Wiley Series in Probability and Statistics).

BISHOP, R. E.; PRICE, W. Hydroelasticity of ships. Cambridge, UK.: Cambridge University Press, 1979.

BISPO, I. B. Aprimoramento de método para inferência de espectro de ondas a partir de movimentos de sistemas oceânicos. Dissertação Mestrado em Engenharia Naval e Oceânica (in Portugese) — Escola Politécnica, Universidade de São Paulo, São Paulo, BR., 2011.

BISPO, I. B. Inferência bayesiana de ondas do mar a partir de movimentos de uma plataforma FPSO: uma nova metodologia de calibração validada com dados de campo e capaz de reduzir erros de estimação. Tese Doutorado em Engenharia Naval e Oceânica (in Portugese) — Escola Politécnica, Universidade de São Paulo, São Paulo, BR., 2011. 
BISPO, I. B.; FILHO, A. N. Q.; TANNURI, E. A.; SIMOS, A. N. Motion-based wave inference: Monitoring campaign on a turret fpso. In: THE 35TH INTERNATIONAL CONFERENCE ON OCEAN, OFFSHORE AND ARCTIC ENGINEERING. OMAE2016. Busan, KR., 2016.

BISPO, I. B.; SIMOS, A. N.; TANNURI, E. A.; CRUZ, J. J. da. Motion-based wave estimation by a bayesian inference method: a procedure for pre-defining the hyperparameters. In: THE 22ND INTERNATIONAL OCEAN AND POLAR ENGINEERING CONFERENCE. ISOPE2012. Rhodes, GD., 2012.

BRETSCHNEIDER, C. L. Wave variability and wave spectra for wind-generated gravity waves. Washington, DC., 1959.

BUI-THANH, T. A gentle tutorial on statistical inversion using the bayesian paradigm. Institute for Computational Engineering and Sciences, Technical Report ICES-12-18, Citeseer, 2012.

BULMER, M. Principles of Statistics. Oxford, UK.: Dover Publications, 2012. (Dover Books on Mathematics).

CAMAS, B. S. A Time-Domain finite Element Method for Seakeeping and Wave Resistance Problems. PhD Thesis in Civil Engineering - Technical University of Madrid, Madrid, ES., 2016.

CHAKRABARTI, S. Handbook of Offshore Engineering (2-volume set). Oxford, UK.: Elsevier, 2005.

CHAPLIN, J. R. Loading on a cylinder in uniform oscillatory flow: Planar oscillatory flow. Applied Ocean Research, Elsevier, v. 10, n. 3, p. 120-128, 1988.

CHEN, Z.; ZEZONG, C.; YANNI, J.; LINGANG, F.; GENGFEI, Z. Exploration and validation of wave-height measurement using multifrequency hf radar. Journal of Atmospheric and Oceanic Technology, v. 30, n. 9, p. 2189-2202, 2013.

CLAUSS, G. Task-related wave groups for seakeeping tests or simulation of design storm waves. Applied Ocean Research, Elsevier, v. 21, n. 5, p. 219-234, 1999.

CLAUSS, G.; SHIELDS, M.; LEHMANN, E.; ÖSTERGAARD, C. Offshore Structures: Volume I: Conceptual Design and Hydromechanics. London, UK.: Springer London, 2014.

CUMMINS, W. The impulse response function and ship motions. Washington, DC., 1962.

DAWID, A. P.; STONE, M.; ZIDEK, J. V. Marginalization paradoxes in bayesian and structural inference. Journal of the Royal Statistical Society. Series B (Methodological), JSTOR, p. 189-233, 1973.

DENIS, M. S.; PIERSON, W. On the motion of ships in confused seas. Transactions SNAME, v. 61, p. 280-354, 1953.

DURRANT, T. H.; GREENSLADE, D. J.; SIMMONDS, I. Validation of jason-1 and envisat remotely sensed wave heights. Journal of Atmospheric and Oceanic Technology, v. 26, n. 1, p. 123-134, 2009. 
ESSEN, S. van; EWANS, K.; MCCONOCHIE, J. Wave buoys performance in short and long waves, evaluated using tests on a hexapod. In: THE 37TH INTERNATIONAL CONFERENCE ON OCEAN, OFFSHORE AND ARCTIC ENGINEERING. OMAE2017. Madrid, ES., 2018.

EWANS, K.; JONATHAN, P. Estimating extreme wave design criteria incorporating directionality. In: 9TH INTERNATIONAL WORKSHOP ON WAVE HINDCASTING \& FORECASTING. Vitoria, CA., 2006. p. 24-29.

FALTINSEN, O. Sea Loads on Ships and Offshore Structures. Cambridge, UK.: Cambridge University Press, 1993. (Cambridge Ocean Technology Series).

FILIPPELLI, M. V.; MARKUS, M.; EBERHARD, M.; H., B. B.; DUBOIS, L. Metocean data needs, assessment and data collection and strategy development for the massachusetts wind energy area. In: AWS TRUEPOWER. Albany, NY., 2015.

FUCILE, F.; LUDENO, G.; SERAFINO, F.; BULIAN, G.; SOLDOVIERI, F.; LUGNI, C. Some challenges in recovering wave features from a wave radar system. In: THE 26TH INTERNATIONAL OCEAN AND POLAR ENGINEERING CONFERENCE. ISOPE2016. Rhodes, GD., 2016.

GARCIA-GABIN, W. Wave bimodal spectrum based on swell and wind-sea components. IFAC-PapersOnLine, Elsevier, v. 48, n. 16, p. 223-228, 2015.

GODA, Y. Random Seas and Design of Maritime Structures. Singapore: World Scientific, 2000. (Advanced series on ocean engineering).

GOOD, I. The estimation of probabilities: An essay on modern bayesian methods. Research Monograph, MIT Press, 2003.

GOOS-BRASIL. Programa Nacional de Boias. 2018. Accessed on: 2018-08-24. Available at: 〈http://www.goosbrasil.org/pnboia/〉.

HADAMARD, J. Lectures on Cauchy's Problem in Linear Partial Differential Equations. New York, NY.: Dover Publications, 2014.

HASSELMANN, D.; DUNCKEL, M.; EWING, J. Directional wave spectra observed during jonswap 1973. Journal of physical oceanography, v. 10, n. 8, p. 1264-1280, 1980.

HAVER, S.; MOAN, T. On some uncertainties related to the short term stochastic modelling of ocean waves. Applied Ocean Research, Elsevier, v. 5, n. 2, p. 93-108, 1983.

HECKMAN, P. E.; MEYERS, G. G. The calculation of aggregate loss distributions from claim severity and claim count distributions. In: PROC. OF THE CASUALTY ACTUARIAL SOCIETY. Arlington, VA., 1983. v. 70, n. 133-134.

HIRAYAMA, T. Real-time estimation of sea spectra based on motions of a running ship-full scale trial. Journal of the Kansai Society of Naval Architects, n. 204, 1987.

HUA, J.; PALMQUIST, M. Wave estimation through ship motion measurement. In: BRITISH MARITIME TECHNOLOGY. London, UK., 1994.

HUBER, P. J. A robust version of the probability ratio test. The Annals of Mathematical Statistics, Institute of Mathematical Statistics, v. 36, n. 6, p. 1753-1758, 1965. 
ISEKI, T. An improved stochastic modeling for bayesian wave estimation. In: THE 31ST INTERNATIONAL CONFERENCE ON OCEAN, OFFSHORE AND ARCTIC ENGINEERING. OMAE2012. Rio de Janeiro, BR., 2012. p. 455-461.

ISEKI, T.; OHTSU, K. Bayesian estimation of directional wave spectra based on ship motions. Control Engineering Practice, Elsevier, v. 8, n. 2, p. 215-219, 2000.

ITTC. Recommended procedures and guidelines in seakeeping experiments. In: SEAKEEPING COMMITTEE OF THE 27TH ITTC. Copenhagen, DK., 2014.

JEON, S.; CHO, Y.; SEO, M.; CHO, J.; JEONG, W. Dynamic response of floating substructure of spar-type offshore wind turbine with catenary mooring cables. Ocean Engineering, Elsevier, v. 72, p. 356-364, 2013.

KITAGAWA, G.; GERSCH, W. Smoothness Priors Analysis of Time Series. New York, NY.: Springer New York, 2012. (Lecture Notes in Statistics).

KONISHI, S.; KITAGAWA, G. Generalised information criteria in model selection. Biometrika, Oxford University Press, v. 83, n. 4, p. 875-890, 1996.

LEE, C.-H.; NEWMAN, J. N. Wamit user manual. WAMIT, Inc, 2006.

LEWIS, E. V. Principles of naval architecture second revision. Jersey: SNAME, 1988.

LIAN, W. Semi-submersible model test. main report. In: MARINTEK. MARINTEK REPORT (Personal communication). Trondheim, NO., 1997.

LINDLEY, D. V.; SMITH, A. F. Bayes estimates for the linear model. Journal of the Royal Statistical Society. Series B (Methodological), JSTOR, p. 1-41, 1972.

LLOYD, A. Seakeeping: ship behaviour in rough weather. Chichester, UK.: E. Horwood, 1989.

LONGUET-HIGGINS, M.; CARTWRIGHT, D.; SMITH, N. Observations of the directional spectrum of sea waves using motions of a floating buoy. Ocean Wave Spectra, Prentice Hall, New York, p. 111-136, 1963.

LONGUET-HIGGINS, M. S. The statistical analysis of a random, moving surface. Philosophical Transactions of the Royal Society of London A: Mathematical, Physical and Engineering Sciences, The Royal Society, v. 249, n. 966, p. 321-387, 1957.

MACKAY, E. B. L. Wave Energy Resource Assessment. PhD Thesis - University of Southampton, Southampton, UK., 2009.

MAGNUSSON, A. K. Forecasting extreme waves in practice. In: PROC. OF THE ROGUE WAVES. Brest, FR., 2008. p. 1-15.

MAS-SOLER, J.; SIMOS, A. N.; MELLO, P. C. de; TANNURI, E. A.; SOUZA, F. L. A preliminary assessment of the use of a large semi-submersible platform as a motion-based wave sensor. In: ASME. ASME 2017 36th International Conference on Ocean, Offshore and Arctic Engineering. [S.l.], 2017. 
MAS-SOLER, J.; SIMOS, A. N.; TANNURI, E. A. Estimating on-site wave spectra from the motions of a semi-submersible platform: An assessment based on model scale results. Ocean Engineering, Elsevier, v. 153, p. 154-172, 2018.

MATHIESEN, M.; NYGAARD, E. Asgard field metocean design basis. In: STATOIL. Metocean Main Report (Personal communication). Trondheim, NO., 2013.

MELLO, P. C. de. Sistema de automação e controle para tanques oceânicos com múltiplos atuadores. Tese Doutorado em Engenharia de Controle e Automação Mecânica (in Portugese) — Escola Politécnica, Universidade de São Paulo, São Paulo, BR., 2005.

MELLO, P. D.; CARNEIRO, M.; TANNURI, E. A.; JR, F. K.; MARQUES, R. P.; ADAMOWSKI, J. C.; NISHIMOTO, K. A control and automation system for wave basins. Mechatronics, Elsevier, v. 23, n. 1, p. 94-107, 2013.

MONTAZERI, N.; NIELSEN, U. D. Parametric estimation in the wave buoy analogy: An elaborated approach based on energy considerations. In: THE 33RD INTERNATIONAL CONFERENCE ON OCEAN, OFFSHORE AND ARCTIC ENGINEERING. OMAE2014. San Francisco, CA., 2014.

MONTAZERI, N.; NIELSEN, U. D.; JENSEN, J. J. Trend modelling of wave parameters and application in onboard prediction of ship responses. In: OCEANS 2015-MTS/IEEE. Washington, DC., 2015. p. 1-8.

MONTAZERI, N.; NIELSEN, U. D.; JENSEN, J. J. Estimation of wind sea and swell using shipboard measurements-a refined parametric modelling approach. Applied Ocean Research, Elsevier, v. 54, p. 73-86, 2016.

MORISON, J.; JOHNSON, J.; SCHAAF, S. The force exerted by surface waves on piles. Journal of Petroleum Technology, Society of Petroleum Engineers, v. 2, n. 05, p. 149-154, 1950.

NAITO, S.; KIHARA, H. Mutual relation between record length and accuracy of measuring data in irregular waves. Journal of the Society of Naval Architects of Japan, The Japan Society of Naval Architects and Ocean Engineers, v. 1993, n. 174, p. 397-408, 1993.

NEWMAN, J. Marine Hydrodynamics. Cambridge, MA.: MIT Press, 1977.

NIELSEN, U. D. Estimations of on-site directional wave spectra from measured ship responses. Marine Structures, Elsevier, v. 19, n. 1, p. 33-69, 2006.

NIELSEN, U. D. Response-based estimation of sea state parameters-influence of filtering. Ocean Engineering, Elsevier, v. 34, n. 13, p. 1797-1810, 2007.

NIELSEN, U. D. Introducing two hyperparameters in bayesian estimation of wave spectra. Probabilistic Engineering Mechanics, Elsevier, v. 23, n. 1, p. 84-94, 2008.

NIELSEN, U. D. A concise account of techniques available for shipboard sea state estimation. Ocean Engineering, Elsevier, v. 129, p. 352-362, 2017.

NIELSEN, U. D. Deriving the absolute wave spectrum from an encountered distribution of wave energy spectral densities. Ocean Engineering, Elsevier, v. 165, p. 194-208, 2018. 
NIELSEN, U. D.; BRODTKORB, A. H.; SØRENSEN, A. J. A brute-force spectral approach for wave estimation using measured vessel motions. Marine Structures, Elsevier, v. 60, p. 101-121, 2018.

NIELSEN, U. D.; GALEAZZI, R.; BRODTKORB, A. H. Evaluation of shipboard wave estimation techniques through model-scale experiments. Monterey, CA., 2016.

NIELSEN, U. dam. Estimation of Directional Wave Spectra from Measured Ship Responses. PhD Thesis in Costal, Maritime and Structural Engineering - Technical university of Denmark, Lyngby, DK., 2005.

NOAA. Data Buoy Center. 2018. Accessed on: 2018-08-24. Available at: 〈https: //www.ndbc.noaa.gov/ $/$.

NWOGU, O. Maximum entropy estimation of directional wave spectra from an array of wave probes. Applied Ocean Research, Elsevier, v. 11, n. 4, p. 176-182, 1989.

OCHI, M. Applied Probability and Stochastic Processes: In Engineering and Physical Sciences. London, UK.: Wiley, 1990. (Wiley Series in Probability and Statistics).

OCHI, M. K.; HUBBLE, E. N. Six-parameter wave spectra. In: Coastal Engineering 1976. [S.l.: s.n.], 1977. p. 301-328.

OLJEMUSEUM, N. Asgard. 2015. Accessed on: 2018-10-25. Available at: 〈https: //www.norskolje.museum.no/en/asgard-2/ $\rangle$.

PETROBRAS. Principais Operações. 2018. Accessed on: 2018-08-24. Available at: $\langle$ http://www.petrobras.com.br/pt/nossas-atividades/principais-operacoes/bacias/ $\rangle$.

PIERSON, J.; WILLARD, J.; MOSKOWITZ, L. A proposed spectral form for fully developed wind seas based on the similarity theory of sa kitaigorodskii. Journal of geophysical research, Wiley Online Library, v. 69, n. 24, p. 5181-5190, 1964.

PINKSTER, J. et al. Wave feed-forward as a means to improve dynamic positioning. In: OFFSHORE TECHNOLOGY CONFERENCE. Offshore Technology Conference. Houston, TX., 1978.

SARPKAYA, T. Wave Forces on Offshore Structures. New York, NY.: Cambridge University Press, 2010. (Wave Forces on Offshore Structures).

SHAW, C.; CHAIRMAN, O.; SOLUTIONS, S. G.; RIJSWIJK, N. Offshore industry requirements and recent metocean technology developments. In: Proc. WMO Workshop on Advances in Marine Climatology (CLIMAR99). Vancuver, CA.: [s.n.], 1999. p. 8-15.

SHIBATA, R. Asymptotic mean efficiency of a selection of regression variables. Annals of the Institute of Statistical Mathematics, Springer, v. 35, n. 1, p. 415-423, 1983.

SHIN, K.; HAMMOND, J. Fundamentals of Signal Processing for Sound and Vibration Engineers. [S.l.]: Wiley, 2008. ISBN 9780470725641.

SIMOS, A. N.; TANNURI, E. A.; CRUZ, J. J. da; FILHO, A. N. Q.; BISPO, I. B. da S.; CARVALHO, R. C. Development of an on-board wave estimation system based on the motions of a moored fpso: Commissioning and preliminary validation. In: THE 
31ST INTERNATIONAL CONFERENCE ON OCEAN, OFFSHORE AND ARCTIC ENGINEERING. OMAE2012. Rio de Janeiro, BR., 2012. p. 259-270.

SIMOS, A. N.; TANNURI, E. A.; SPARANO, J. V.; MATOS, V. L. Estimating wave spectra from the motions of moored vessels: Experimental validation. Applied Ocean Research, Elsevier, v. 32, n. 2, p. 191-208, 2010.

SOARES, C.; FRICKE, W. Advances in Marine Structures. London, UK.: CRC Press, 2011. (Balkema book).

SOBOL, I. M. A primer for the Monte Carlo method. Boca Raton, FL.: CRC press, 1994.

SOUZA, F. L. de; TANNURI, E. A.; MELLO, P. C. de; FRANZINI, G.; MAS-SOLER, J.; SIMOS, A. N. Bayesian estimation of directional wave-spectrum using vessel motions and wave-probes: Proposal and preliminary experimental validation. Journal of Offshore Mechanics and Arctic Engineering, ASME, v. 140, n. 4, 2018.

STANSBERG, C. T. On the fourier series decomposition of directional wave spectra. In: THE 8TH INTERNATIONAL OFFSHORE AND POLAR ENGINEERING CONFERENCE. ISOPE1998. Montréal, CA., 1998.

STATOIL. Asgard: Statoil-operated fields in Norway. 2014. Accessed on: 2016-12-19. Available at: 〈http://www.statoil.com/en/ouroperations/explorationprod/ncs/aasgard/ Pages/default.aspx $\rangle$.

STEIN, C. An example of wide discrepancy between fiducial and confidence intervals. The Annals of Mathematical Statistics, JSTOR, v. 30, n. 4, p. 877-880, 1959.

TANnURI, E. A.; MELlO, P. C.; SALES, J. S. J.; SIMOS, A. N.; MATOS, V. Estimation of directional wave spectrum using a wave-probe array. In: PROC. OF 3RD INT. WORKSHOP ON APPLIED OFFSHORE HYDRODYNAMICS. Rio de Janeiro, BR., 2007.

TANNURI, E. A.; SPARANO, J. V.; SIMOS, A. N.; CRUZ, J. J. D. Estimating directional wave spectrum based on stationary ship motion measurements. Applied Ocean Research, Elsevier, v. 25, n. 5, p. 243-261, 2003.

TECHNOLOGIES, A. AXYS metocean buoys. 2018. Accessed on: 2018-10-25. Available at: 〈http://axystechnologies.com/solutions/oil-gas-environmental-monitoring/ $\rangle$.

TOFFOLI, A.; ONORATO, M.; BITNER-GREGERSEN, E.; MONBALIU, J. Development of a bimodal structure in ocean wave spectra. Journal of Geophysical Research: Oceans, Wiley Online Library, v. 115, n. C3, 2010.

TORSETHAUGEN, K. A two peak wave spectrum model. In: THE $12 \mathrm{TH}$ INTERNATIONAL CONFERENCE ON OCEAN, OFFSHORE AND ARCTIC ENGINEERING. OMAE1993. Glasgow, UK., 1993.

TORSETHAUGEN, K.; HAVER, S. Simplified double peak spectral model for ocean waves. In: THE 14TH INTERNATIONAL OFFSHORE AND POLAR ENGINEERING CONFERENCE. ISOPE2004. Toulon, FR., 2004. 
TUCKER, M. The analysis of finite-length records of fluctuating signals. British Journal of Applied Physics, IOP Publishing, v. 8, n. 4, p. 137, 1957.

TUCKER, M.; CHAllenOR, P. G.; CARTER, D. Numerical simulation of a random sea: a common error and its effect upon wave group statistics. Applied ocean research, Elsevier, v. 6, n. 2, p. 118-122, 1984.

WALKER, S. G. Bayesian information in an experiment and the fisher information distance. Statistics $\&$ Probability Letters, Elsevier, v. 112, p. 5-9, 2016.

WEBSTER, W. C.; DILLINGHAM, J. T. Determination of directional seas from ship motions. 1981.

WHITTAKER, E. T. On a new method of graduation. Proceedings of the Edinburgh Mathematical Society, Cambridge University Press, v. 41, p. 63-75, 1922.

WIJAYA, T. Can be a semisubmersible platform be used as a wave sensor? Master Thesis in Marine Technology - Norwegian University of Science and Technology, Trondheim, NO., 2009.

ZELLNER, A. Optimal information processing and bayes's theorem. The American Statistician, Taylor \& Francis Group, v. 42, n. 4, p. 278-280, 1988. 


\section{APPENDIX A - SINUSOIDAL WAVE AS A RANDOM PROCESS}

For a given amplitude, frequency and wave number, the randomness of a sinusoidal wave can be introduced by defining the phase shift, $\phi$, with respect to a given frequency as a random variable taking values in the range $[0,2 \pi)$. When modeling ocean wave trains, it is commonly assumed that $\phi$ has a constant density thus implying that all phase shift values have the same probability of occurrence (LONGUET-HIGGINS, 1957).

Accordingly, the probability distribution of the phase shift (see Eq. (3.13)) can be written as,

$$
P(\varphi<\phi<\varphi+\mathrm{d} \varphi)=\operatorname{pdf}(\phi) \mathrm{d} \phi=\frac{1}{2 \pi} \mathrm{d} \phi
$$

obtaining that,

$$
\int_{-\infty}^{+\infty} \operatorname{pdf}(\phi) \mathrm{d} \phi=\int_{-\infty}^{+\infty} \frac{1}{2 \pi} \mathrm{d} \phi=1
$$

Same instantaneous value of the sinusoidal wave can be obtained using two different phases. In other words, for a same time instant two different phase shifts can provide the same instantaneous value. From this property, the probability density function of the wave elevation is given by,

$$
\operatorname{pdf}(\eta) \mathrm{d} \eta=P(\eta=\eta)=P\left(\phi=\phi_{1}\right)+P\left(\phi=\phi_{2}\right)=2 \operatorname{pdf}(\phi) \mathrm{d} \phi=\frac{\mathrm{d} \phi}{\pi}
$$

This is an important relation that allows the following variable change (considering the fact that the probability density function should be always positive), 


$$
\operatorname{pdf}(\eta)=\operatorname{pdf}(\phi)\left|\frac{\mathrm{d} \phi}{\mathrm{d} \eta}\right|=\frac{1}{\pi}\left|\frac{\mathrm{d} \phi}{\mathrm{d} \eta}\right|
$$

Thus, $\mathrm{d} \eta$ should be determined in order to estimate the probability density function of the amplitude of the wave. Thus simplifying Eq. (3.13) for an unidirectional wave and differentiating,

$$
\mathrm{d} \eta=-A \sin (\mathrm{k} x-\omega t+\phi) \mathrm{d} \phi,
$$

relation that should be replaced in Eq. (A.6), leading to the expression of the probability density function of the amplitude of the sinusoidal wave,

$$
\operatorname{pdf}(\eta)=\frac{1}{\pi\left(A^{2}-\eta^{2}\right)^{\frac{1}{2}}}
$$

Now, by means of the expression provided in Eq. (A.6), the statistical characteristics of the process can be computed as follows. In the first place, the mean value is given by,

$$
E[\eta(x, t)]=\int_{-\infty}^{+\infty} \eta \operatorname{pdf}(\eta) \mathrm{d} \eta=\int_{-\infty}^{+\infty} \frac{\eta}{\pi\left(A^{2}-\eta^{2}\right)^{\frac{1}{2}}} \mathrm{~d} \eta=0 .
$$

This result shows that the process is independent of the time and space (GODA, 2000). Secondly, the mean square can be computed as,

$$
E\left[\eta^{2}(x, t)\right]=\int_{-\infty}^{+\infty} \eta^{2} \operatorname{pdf}(\eta) \mathrm{d} \eta=\int_{-\infty}^{+\infty} \frac{\eta^{2}}{\pi\left(A^{2}-\eta^{2}\right)^{\frac{1}{2}}} \mathrm{~d} \eta=\frac{a^{2}}{2} .
$$

relation that shows that the mean square does not depend of the space and time. In the third place, the autocorrelation function is defined by the expression provided in Eq. (A.9). For this purpose, assume that the random process under study produces two random variables $\eta\left(x_{1}, t_{1}\right)$ and $\eta\left(x_{2}, t_{2}\right)$ at times an positions $\left(x_{1}, t_{1}\right)$ and $\left(x_{2}, t_{2}\right)$, respectively (ÅSTRÖM, 2012). 


$$
R_{\eta \eta}:=R_{\eta \eta}\left(x_{1}, t_{1} ; x_{2}, t_{2}\right)=E\left[\eta\left(x_{1}, t_{1}\right) \eta\left(x_{2}, t_{2}\right)\right]=\int_{-\infty}^{+\infty} \eta_{1} \eta_{2} \operatorname{pdf}_{\eta\left(x_{1}, t_{1}\right) \eta\left(x_{2}, t_{2}\right)}\left(\eta_{1}, \eta_{2}\right) \mathrm{d} \eta_{1} \mathrm{~d} \eta_{2}
$$

The resulting relation, after some algebra, can be rewritten as,

$$
R_{\eta \eta}=E\left[\frac{A^{2}}{2}\left(\cos \left(\mathrm{k}\left(x_{1}+x_{2}\right)-\omega\left(t_{1}+t_{2}\right)+2 \phi\right)+\cos \left(\mathrm{k}\left(x_{1}-x_{2}\right)-\omega\left(t_{1}-t_{2}\right)\right)\right)\right]
$$

and using the following definitions $\bar{\rho}=x_{1}-x_{2}, \tau=t_{1}-t_{2}, x_{3}=x_{1}+x_{2}$ and $t_{3}=t_{1}+t_{2}$, the autocorrelation function will be,

$$
R_{\eta \eta}=\frac{A^{2}}{2} E\left[\left(\cos \left(\mathrm{k} x_{3}-\omega t_{3}+2 \phi\right)+a \cos (\mathrm{k} \bar{\rho}-\omega \tau)\right)\right]
$$

Another important property of the sinusoidal wave is that the mean value over one complete cycle is zero. This feature allows the simplification of the previous equation, being reduced to,

$$
R_{\eta \eta}=\frac{A^{2}}{2} \cos (\mathrm{k} \bar{\rho}-\omega \tau)
$$

Equation A.12 shows that autocorrelation function is independent of the time and space and, as a consequence, it only depends on the relative values of the time and position with respect to a certain reference. Also, it has been shown that the sinusoidal wave is a stationary process regarding its mean value, the mean square and the autocorrelation function. Therefore, the process can be assumed ergodic.

Finally, another example is provided for illustration purposes. Let be $\eta(x, t)$ a random process that is given as the sum of two independent sinusoidal waves,

$$
\begin{aligned}
& \eta_{1}(x, t)=A_{1} \cos \left(\mathrm{k}_{1} x-\omega_{1} t+\phi_{1}\right), \\
& \eta_{2}(x, t)=A_{2} \cos \left(\mathrm{k}_{2} x-\omega_{2} t+\phi_{2}\right),
\end{aligned}
$$

Thus, 


$$
\eta(x, t)=A_{1} \cos \left(\mathrm{k}_{1} x-\omega_{1} t+\phi_{1}\right)+A_{2} \cos \left(\mathrm{k}_{2} x-\omega_{2} t+\phi_{2}\right)
$$

Leading to the mean value,

$$
E[\eta(x, t)]=E\left[\eta_{1}(x, t)+\eta_{2}(x, t)\right]=E\left[\eta_{1}(x, t)\right]+E\left[\eta_{2}(x, t)\right]=0+0=0 .
$$

Accordingly, the mean square is computed as follows,

$$
\begin{aligned}
& E\left[\eta^{2}(x, t)\right]=E\left[\left(\eta_{1}(x, t)+\eta_{2}(x, t)\right)^{2}\right]= \\
& E\left[\eta_{1}^{2}(x, t)\right]+E\left[\eta_{2}^{2}(x, t)\right]+2 E\left[\eta_{1}(x, t) \eta_{2}(x, t)\right],
\end{aligned}
$$

If both process are independent, the resulting covariance must be zero $\left(E\left[\eta_{1}(x, t) \eta_{2}(x, t)\right]=\right.$ $0)$, also, as it has been shown before, the mean value of the process over one period is equal to zero. As a consequence, the Eq. (A.16) is reduced to,

$$
E\left[\eta^{2}(x, t)\right]=\frac{A_{1}^{2}}{2}+\frac{A_{2}^{2}}{2}
$$

Accordingly, the autocorrelation function can be reduced to the following expression,

$$
\begin{aligned}
& R_{\eta \eta}=R_{\eta \eta}\left(x_{1}, t_{1} ; x_{2}, t_{2}\right)=E\left[\eta\left(x_{1}, t_{1}\right) \eta\left(x_{2}, t_{2}\right)\right]= \\
& R_{11}+R_{22}+R_{12}=R_{11}+R_{22}
\end{aligned}
$$

Finally, this process presents the same characteristics regarding the mean value, the mean square and the autocorrelation function as the sinusoidal wave, i.e. the process is stationary and homogeneous and it can be assumed ergodic. In this context ergodic means that the number of the cosine waves included in the analysis is sufficiently large.

The foregoing results present a basic illustration of the main fundamentals that allow the characterization of the sea surface elevation using the statistical approach. Furthermore, an interesting result of this approach arises when infinite waves components are included, thus the well-known Longuet-Higgins model (see eq. (3.13)) is obtained. 


\section{APPENDIX B - STATISTICAL DESCRIPTION OF WAVES}

A brief discussion regarding key concepts of the probabilistic description of the sea is included herein. Therefore, conditions concerning the Gaussianity, Ergodicity, Stationarity and optimal sample time of the process are reviewed.

\section{B.1 Gaussianity}

In the Longuet-Higgins random sea model, the sea surface profile is obtained as a result of the sum of infinity sinusoidal wave components (LONGUET-HIGGINS, 1957). Examination of the resulting amplitudes of this model reveals that this process can be assumed stationary Gaussian, as a direct consequence of the central limit theorem. On the other hand, it is common to assume the narrow banded spectrum hypothesis, in order to assure that there is a very small probability that the maxima of the irregular wave profile are located elsewhere than at the wave crests. Accordingly, the amplitude of the wave components gives the amplitude of the wave envelope. Nonetheless, Longuet-Higgins (1957) shows that for a Gaussian process, the envelope of an irregular wave profile can always be defined, even if the spectrum is not narrow.

Another remark should be done regarding narrow banded processes, as it is the resulting sea of the Longuet-Higgins random sea model. This kind of processes has an important relationship with the Rayleigh distribution, which describes the probability distribution of the the envelope of the random data series. The Rayleigh probability distribution can be derived from the Gaussian distribution, as it shown in (BENDAT; PIERSOL, 2010). A direct consequence of the use of the Rayleigh distribution to describe the envelope of the 
irregular wave profile is that important information about the statistical characteristics of the sea spectrum can be derived from this distribution, as it has been shown in chapter 3 .

Concerning the effectiveness of the Gaussianity assumption, chapter 4 provides a detailed review about the Gaussian characteristics of the wave elevation and the implications on the Bayesian inference approach. This analysis shows that it is expected that the Guassianity assumption will be no longer valid for processes that are highly non-stationary or/and when the non-linear terms of the hydrodynamic equations become significant. Furthermore, when irregular sea conditions are generated artificially by means of numerical simulations or in controlled conditions, such as wave basins, the relation between different sinusoidal wave components of the Longet-Higgins model may lead to a non-gaussian process, see (TUCKER; CHALLENOR; CARTER, 1984). However, the Gaussianity assumption leads to very attractive simplifications from a practical point of view, and, as a consequence, the Gaussianity hypothesis is commonly adopted even for non-linear processes.

In order to illustrate these points Fig. B.1 and Fig. B.2 provide the empirical distribution of the surface elevation for two different sea conditions tested in the wave basin (a detailed description of all conditions tested during the experimental campaign is provided in chapter 5). The sea conditions selected for this analysis correspond to: The first sea condition, with $T_{p}=9.5 \mathrm{~s}$ and $H_{s}=3.5 \mathrm{~m}$ (mild sea state), and the most extreme sea condition, with $T_{p}=19.5 \mathrm{~s}$ and $H_{s}=16.4 \mathrm{~m}$ (once in 100 years). Both sea conditions were obtained using a JONSWAP spectrum. Sea states generated by means of a Torsethaugen spectrum are assumed not proper for this analysis because their spectrum band is larger than the equivalent JONSWAP spectrum and, as a consequence, the expected deviation from the Gaussian distribution could be larger. The test conditions analyzed here indicate some discrepancies of the surface elevation from the normal distribution. Also, in order to provide some statistical framework, various parametric distributions have been adjusted to the empirical data and included in these figures, i.e.: Normal (gaussian), GEV (Generalized Extreme Values), Logistic and Tlocationscale distributions, that are extensively documented in the literature.

The probability distributions shown in Fig. B.1 and Fig. B.2 provide a general idea regarding the main differences between the empirical distribution and the adjusted normal 


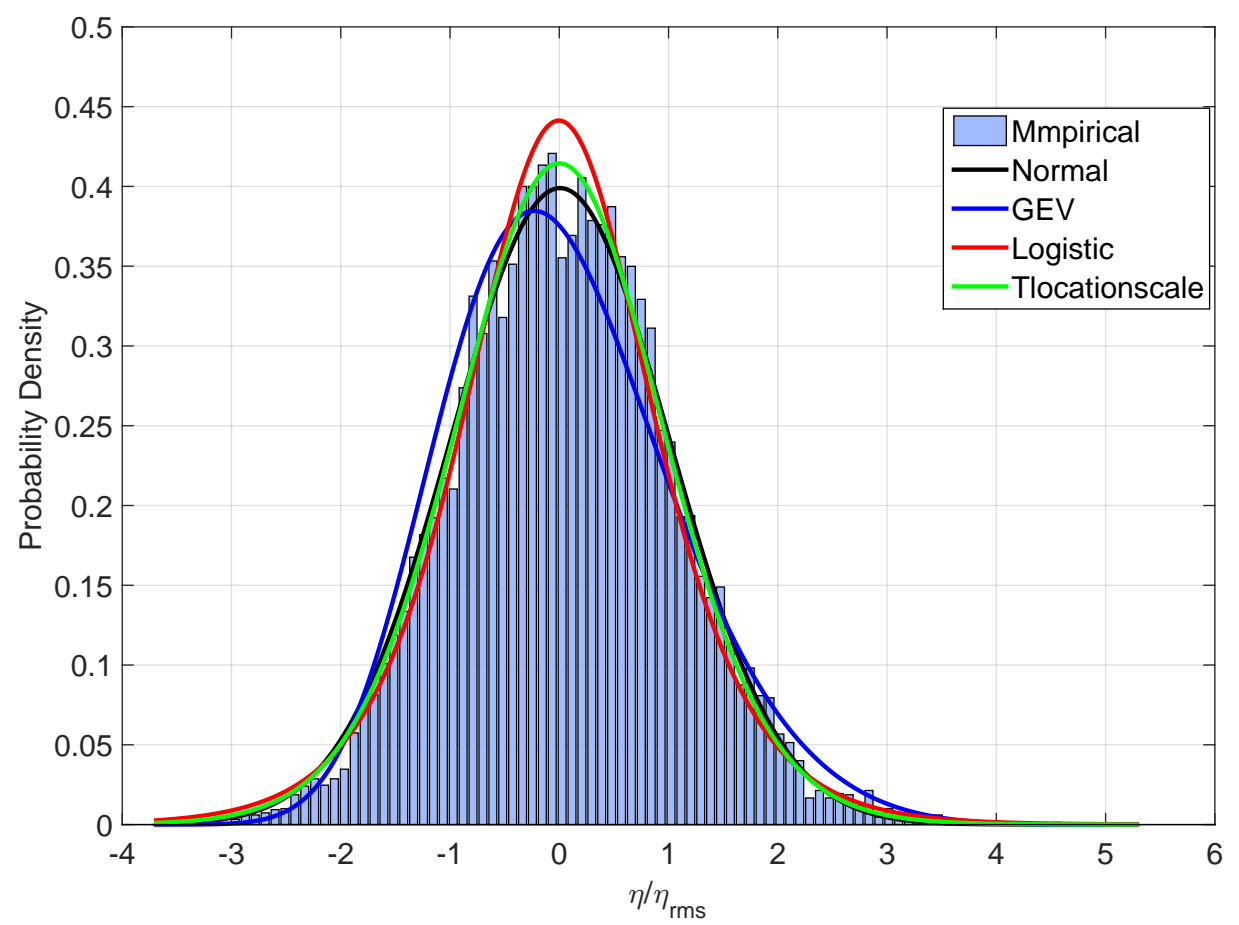

Figure B.1: Distribution of the surface for the first sea condition.

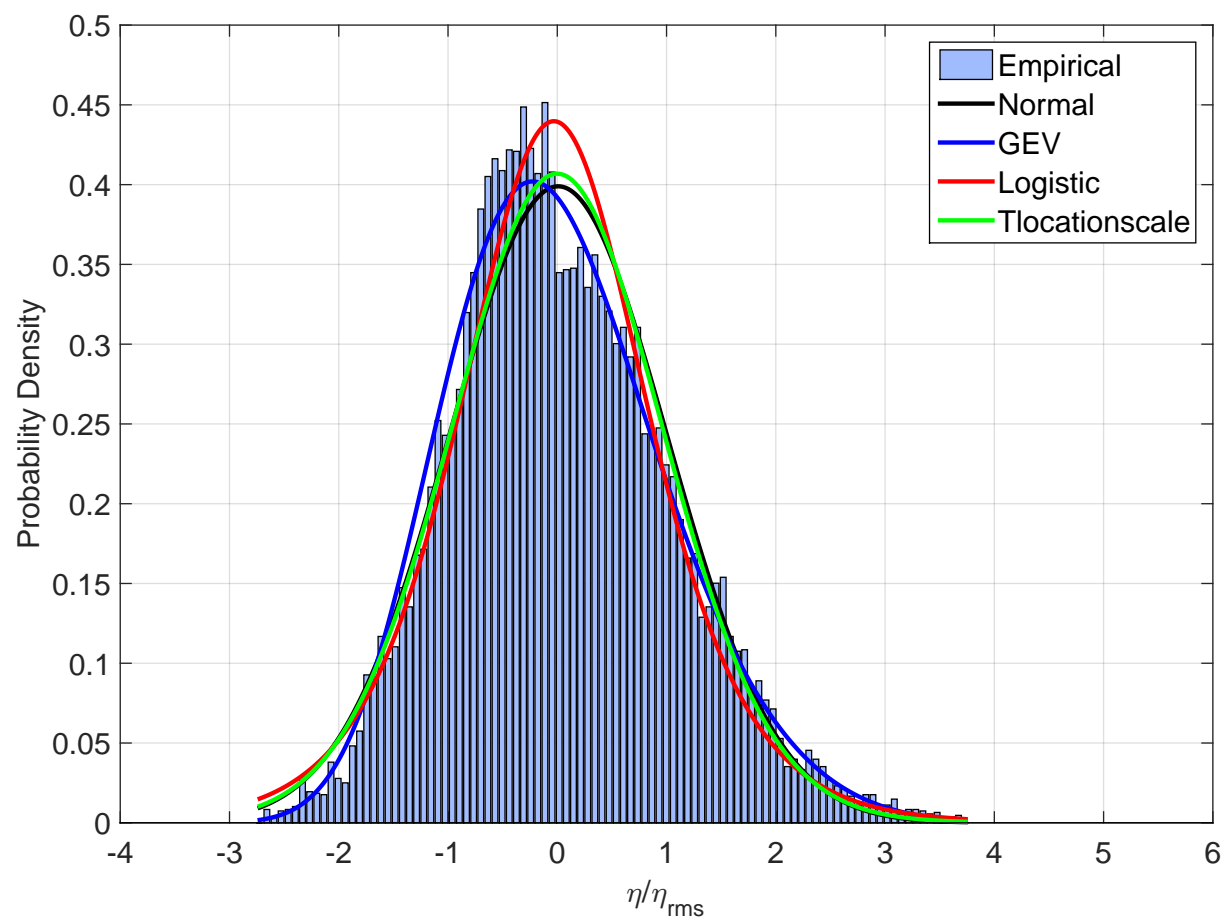

Figure B.2: Distribution of the surface for the once in 100 years sea condition.

distribution. However, a quantitative analysis is needed in order to evaluate the validity of the Gaussianity assumption regarding the sea conditions generated in the wave basin. For 
this purpose (BULMER, 2012) suggests the evaluation of the skewness, which provides a measurement of the asymmetry of the measured probability distribution.

Concerning the normal distribution, it is characterized by having an skewness equal to zero, which implies that the data is perfectly symmetric, but this value of the skewness is highly unusual for empirical data. Therefore, the classification proposed in (BULMER, 2012) will be adopted to determine if a the distribution can be assumed symmetric and consequently Gaussian. The criterion adopted sates that:

- If the skewness is less than -1 or greater than +1 , the distribution is assumed as highly skewed;

- If the value of the skewness is between -1 and -0.5 or between +0.5 and +1 , the estimated distribution will be assumed moderately skewed;

- If the skewness is between -0.5 and +0.5 , the measured distribution can be assumed approximately symmetric.

The values of the estimated skewness of the measured wave elevation for all the sea conditions tested in the wave basin are provided in Tab. B.1. These results show that all the sea conditions tested in the wave basin present skewness values between 0.2 and 0.49 . Therefore, the distributions can be assumed approximately symmetric and, consequently, the Gaussianity assumption will be adopted for all the sea conditions tested. Also, it is interesting to remark that the positive value of the skewness, obtained for all the sea conditions, suggests that the wave crest heights are larger than the wave trough depths.

\section{B.2 Ergodicity}

An ensemble of wave profiles is assumed an ergodic process if a particular sample has the property that its time averaged statistics are equal to those of the ensemble average. Therefore, the assumption of ergodicity allows a theoretically correct substitution of ensemble averaging by temporal average (BENDAT; PIERSOL, 2010). It is important to highlight that the hypothesis of ergodicity derives from the linear sea wave theory, as well as the Gaussian hypothesis (see appendix A). 
Table B.1: Estimated skewness for all the sea tests conditions.

\begin{tabular}{ccc} 
Wave ID & Sea Spectrum & Skewness \\
\hline STA-IRR-01 & JONSWAP & 0.31 \\
STA-IRR-02 & JONSWAP & 0.21 \\
STA-IRR-03 & TORSETHAUGEN & 0.25 \\
STA-IRR-04 & TORSETHAUGEN & 0.22 \\
STA-IRR-05 & TORSETHAUGEN & 0.28 \\
STA-IRR-06 & JONSWAP & 0.33 \\
STA-IRR-07 & JONSWAP & 0.23 \\
STA-IRR-08 & TORSETHAUGEN & 0.27 \\
STA-IRR-09 & TORSETHAUGEN & 0.49 \\
STA-IRR-10 & JONSWAP & 0.33 \\
STA-IRR-11 & JONSWAP & 0.24 \\
STA-IRR-12 & TORSETHAUGEN & 0.33 \\
STA-IRR-13 & JONSWAP & 0.33 \\
STA-IRR-14 & TORSETHAUGEN & 0.39 \\
STA-IRR-15 & JONSWAP & 0.46 \\
STA-IRR-16 & JONSWAP & 0.33 \\
STA-IRR-17 & TORSETHAUGEN & 0.38 \\
STA-IRR-18 & JONSWAP & 0.44 \\
STA-IRR-19 & JONSWAP & 0.49 \\
STA-IRR-20 & JONSWAP & 0.36 \\
STA-IRR-21 & TORSETHAUGEN & 0.38 \\
STA-IRR-22 & TORSETHAUGEN & 0.32 \\
STA-IRR-23 & JONSWAP & 0.40 \\
STA-IRR-24 & JONSWAP & 0.48 \\
STA-IRR-25 & JONSWAP & 0.38 \\
STA-IRR-26 & TORSETHAUGEN & 0.39 \\
STA-IRR-27 & JONSWAP & 0.46 \\
STA-IRR-28 & JONSWAP & 0.40 \\
STA-IRR-29 & TORSETHAUGEN & 0.42 \\
STA-IRR-30 & JONSWAP & 0.46 \\
STA-IRR-31 & JONSWAP & 0.40 \\
STA-IRR-32 & TORSETHAUGEN & 0.45 \\
\hline & & \\
\hline
\end{tabular}

Consequently, if the sea surface elevation is an ergodic process, then all the joint pdfs can be estimated from one single realization of the process having infinite time length. So, a process is said to be ergodic if the statistics of a particular sample $\eta_{i}(t)$ are equal to those of the ensemble average,

$$
E[\eta(t)]=\overline{\eta_{i}(t)}=\lim _{T \rightarrow \infty} \frac{1}{T} \int_{0}^{T} \eta_{i}(\tau) \mathrm{d} \tau,
$$




$$
E[\eta(t+\delta t) \eta(t)]=\overline{\eta_{i}(t+\delta t) \eta_{i}(t)}=\lim _{T \rightarrow \infty} \frac{1}{T} \int_{0}^{T} \eta_{i}(\tau+\delta t) \eta_{i}(\tau) \mathrm{d} \tau
$$

It is seen from Eqs. (B.1) and (B.2) that an ergodic process must necessarily be stationary. If these equations hold for a Gaussian process, then all the joint distributions can be obtained and the process will be fully determined. Finally, a stochastic process that possesses ergodicity is always a stationary process. However, the stationarity conditions does not imply ergodicity of the process.

\section{B.3 Stationarity}

Above, the Gaussian and ergodic conditions of random sea waves have been reviewed. This section aims at explaining the stationarity condition, which refers to the characteristic of a stochastic process in which all the statistic properties are time-invariant. For example, if the stationary condition is applied to a certain register of wave elevation, then the arithmetic mean and the autocorrelation function have the following properties:

$$
\begin{gathered}
E[\eta(t)]=\mu_{\eta}=E[\eta(0)], \quad \text { with } \quad-\infty<t<\infty, \\
E[\eta(t+\delta t) \eta(t)]=R_{\eta \eta}=E[\eta(\tau) \eta(0)], \quad \text { with } \quad-\infty<t<\infty .
\end{gathered}
$$

\section{B.4 Optimal sample time}

In theory, the fulfillment of three conditions described above would be sufficient to select the test duration in which a minimum number of relevant events is obtained. However, from a practical point of view this is usually an unrealistic objective, because the wave elevation, even in a controlled experimental campaign at a wave basin, is in general non-stationary. Also, a minimal number of cycles (or waves) is needed (ITTC, 2014) to obtain a statistical significance of the characteristics of the wave spectrum. The use of the well-known Fast Fourier Transform procedure implies that the maximum time duration is given by $T_{\max }=\frac{1}{\Delta f}$, where $\Delta f$ is the frequency separation of the harmonics (see (CAMAS, 2016)). This is due to the fact that wave record repeats itself after this time. 
Another advantage that derives from the adoption of the Fast Fourier Transform is that it allows the estimation of the standard error, $\sigma_{m}^{2}$, that a spectrum presents over the frequency interval of interest (given by $N$ harmonics in the frequency range $\left[f_{m}, f_{m+N-1}\right]$ ), in accordance with the sample time duration of the signal.

In order to deduce the value of the standard error, the random nature of the amplitude of each harmonic of a power spectrum (that has been introduced in section 3.2) should be considered. In the analysis of a single record, the measured value of the power of a given harmonic component is given by $s_{\eta \eta_{i}}$, and as a result of its randomness its value may differ from one to another independent record of the same signal. Nonetheless, it is possible to adopt the average of the amplitude of a given harmonic, $S_{\eta \eta_{i}}$, over many independent records of the same signal as the truly characteristic amplitude of the harmonic (TUCKER, 1957).

For the $j^{\text {th }}$ realization, the average of the spectrum from the $m^{\text {th }}$ to the $(m+N-1)^{t h}$ harmonic is $\left(s_{\eta \eta_{m}}+\ldots+s_{\eta \eta_{m+N-1}}\right) / N$ and the error is the amount that differs from the average of the averaged values of the spectrum over many records $\left(S_{\eta \eta_{m}}+\ldots+S_{\eta \eta_{m+N-1}}\right) / N$. Now, the square of the error can be defined as,

$$
\begin{aligned}
& \frac{\left[\left(S_{\eta \eta_{m}}-s_{\eta \eta_{m}}\right)+\ldots+\left(S_{\eta \eta_{m+N-1}}+s_{\eta \eta_{m+N-1}}\right)\right]}{N^{2}}= \\
& \frac{\left[\sum_{i=m}^{m+N-1}\left(S_{\eta \eta_{i}}-s_{\eta \eta_{i}}\right)+\sum_{n \neq l}\left(S_{\eta \eta_{n}}-s_{\eta \eta_{n}}\right)\left(S_{\eta \eta_{l}}-s_{\eta \eta_{l}}\right)\right]}{N^{2}}
\end{aligned}
$$

By definitions the value computed as the average value of Eq. (B.5) over many analyses is the square of the standard error, $\sigma$. Tucker (1957) shows that the values of $\left(S_{\eta \eta_{n}}-s_{\eta \eta_{n}}\right)$ and $\left(S_{\eta \eta_{l}}-s_{\eta \eta_{l}}\right)$ are unrelated and that their product averaged over many analyses tends to zero. Thus, the standard error can be defied as,

$$
\sigma^{2}=\frac{\sum_{i=m}^{m+N-1}\left(S_{\eta \eta_{i}}-s_{\eta \eta_{i}}\right)}{N^{2}}=\frac{\sum_{i=m}^{m+N-1} \sigma_{i}^{2}}{N^{2}}
$$

Assuming that all the values of the spectrum are equal over the frequency range of interest all the values of $\sigma_{i}^{2}$ and $\sigma^{2}=\frac{\sigma_{i}^{2}}{N}$. Now, from the relation between the variance 
and the expected value of the spectrum in each frequency, obtained in section 3.2 , the standard error can be rewritten as,

$$
\sigma^{2}=\frac{S_{\eta \eta_{i}}^{2}}{N}
$$

Thus, the proportional standard error $\frac{\sigma^{2}}{S_{\eta \eta_{i}}}=\frac{1}{\sqrt{N}}$. As an illustration of the importance of this relation, consider the analysis of a recorded signal characterized by bandwidth equivalent to $0.5 \mathrm{~Hz}$. If a $2.5 \%$ standard error is required, then the signal recorded must have at least 1600 cycles, that is, the record must be 3200s.

This shows that the procedure provides an optimal solution regarding the time duration of the recorded signal if the true power spectrum is uniform and narrowband. However, for different sea conditions it may present some problems. For example, consider the spectra provided in Fig. B.3. In this figure the Torsethaugen spectrum is named as TH and Measured provides the full-scale measured sea spectrum. In this case, if the relations presented above are followed, the total time for the recorded signal will be the same for both spectra: Torsethaugen and the measured. However, from the same figure, it can be observed that the expected mean wave period is different for these spectra. Thus, if the same recording time is considered, the expected number of cycles (or waves) for these sea conditions will be (sufficiently) different and, consequently, the statistical estimators will have distinct significance. Accordingly, a different procedure to determine the time length of the signal is needed to obtain recordings that have the same statistical significance.

The main idea followed in this work, to determine the time duration of the register, is to estimate the variance of a temporal statistical estimator, such as the temporal mean or the temporal mean square of the wave elevation, and set the estimated variance of the estimator less than or equal of a certain value, for all the sea conditions. Therefore, the total recorded time for the tested sea conditions will be different in each case. In order to guarantee a minimum number of cycles, a minimum time span of 30 minutes is considered, that is typically assumed as the length of the stationary period (SOARES; FRICKE, 2011). Then, the variance of the estimator is obtained every minute until a certain sample time is reached, where the computed variance of the estimator is less than 


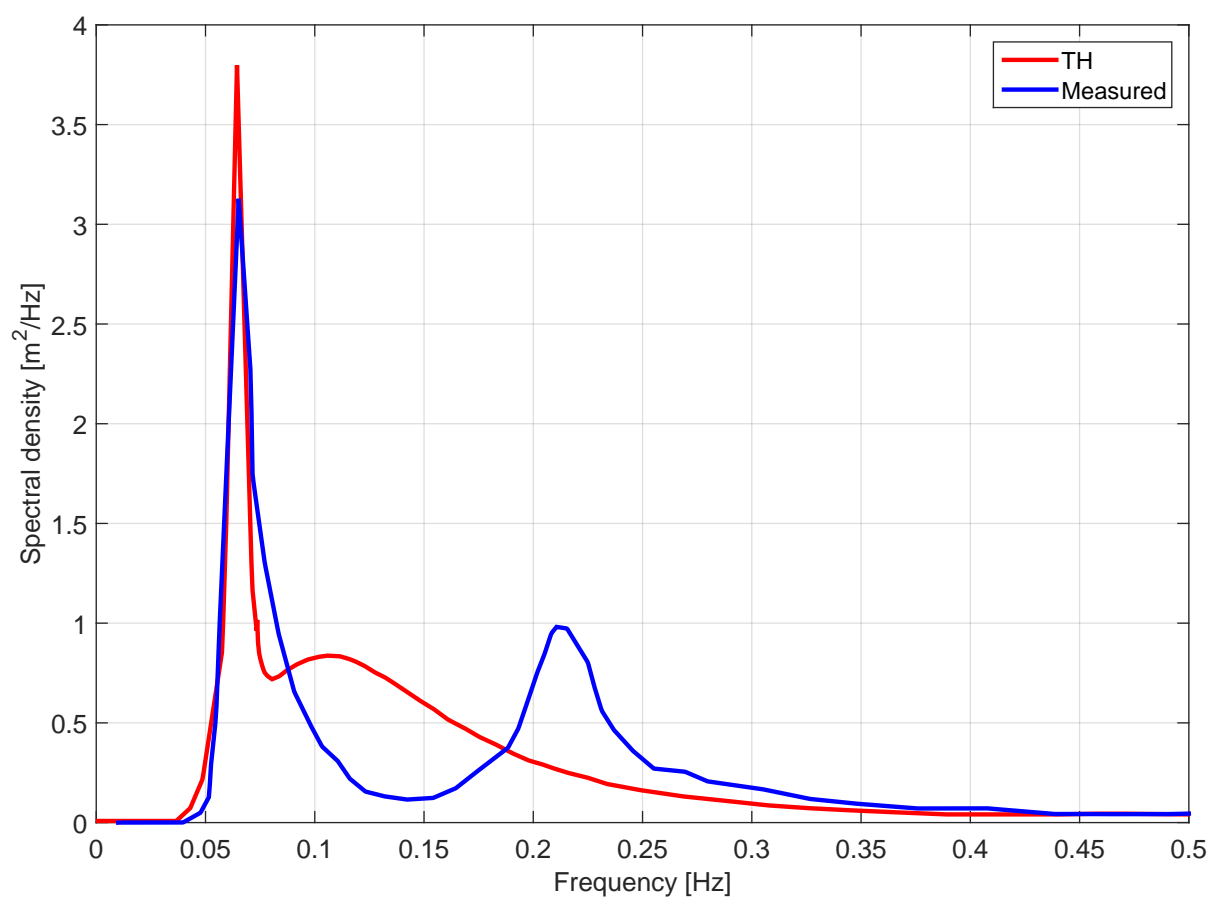

Figure B.3: Illustration of different types of spectra. Adapted from (EWANS; JONATHAN, 2006).

or equal to a prescribed value.

The deduction regarding the estimation of the variance of the running statistical estimators presented below is already reported in (NAITO; KIHARA, 1993) in a similar way. The running mean and running mean square of a process, $\eta$, are given by:

$$
\begin{aligned}
\langle\eta\rangle & =\frac{1}{t} \int_{0}^{t} \eta(\tau) \mathrm{d} \tau, \\
\left\langle\eta^{2}\right\rangle & =\frac{1}{t} \int_{0}^{t} \eta^{2}(\tau) \mathrm{d} \tau .
\end{aligned}
$$

If the process under analysis, $\eta$, is supposed weakly stationary, it has the following properties:

$$
E[\eta]=\int_{-\infty}^{+\infty} \eta \operatorname{pdf}(\eta) \mathrm{d} \eta=\mu_{\eta}
$$




$$
\begin{gathered}
E\left[\eta^{2}\right]=\int_{-\infty}^{+\infty} \eta^{2} \operatorname{pdf}(\eta) \mathrm{d} \eta=\mu_{\eta}^{2}+\sigma_{\eta}^{2}, \\
E[\eta(t) \eta(t+\delta t)]=R_{\eta \eta}(\tau) .
\end{gathered}
$$

From Eqs. (B.8) and (B.9), the expected values of the running mean and the running mean square are:

$$
\begin{gathered}
E[\langle\eta\rangle]=\frac{1}{t} \int_{0}^{t} E[\eta(\tau)] \mathrm{d} \tau=\mu_{\eta}, \\
E\left[\left\langle\eta^{2}\right\rangle\right]=\frac{1}{t} \int_{0}^{t} E\left[\eta^{2}(\tau)\right] \mathrm{d} \tau=\mu_{\eta}+\sigma_{\eta}^{2} .
\end{gathered}
$$

Therefore, the unbiased estimators, for the expected values of $\eta$ and $\eta^{2}$, are given by the running mean and the running mean square. However, the main interest in this case is the degree of variation of these estimators over the time. Thus,

$$
\begin{aligned}
\operatorname{Var}(\langle\eta\rangle)=E\left[\langle\eta\rangle^{2}\right]-E^{2}[\langle\eta\rangle]= & \frac{2}{t^{2}} \int_{0}^{t}\left\{\int_{0}^{\tau}\left(R_{\eta \eta}(\tau)-\mu_{\eta}^{2}\right) \mathrm{d} \tau\right\} \mathrm{d} \tau= \\
& \frac{2}{t} \int_{0}^{t}\left(1-\frac{\tau}{t}\right)\left(R_{\eta \eta}(\tau)-\mu_{\eta}^{2}\right) \mathrm{d} \tau,
\end{aligned}
$$

And following a similar procedure,

$$
\begin{aligned}
\operatorname{Var}\left(\left\langle\eta^{2}\right\rangle\right)=E\left[\left\langle\eta^{2}\right\rangle^{2}\right]-E^{2}\left[\left\langle\eta^{2}\right\rangle\right]= & \frac{2}{t^{2}} \int_{0}^{t}\left\{\int_{0}^{\tau}\left(R_{\eta^{2} \eta^{2}}(\tau)-\left(\sigma_{\eta}^{2}+\mu_{\eta}^{2}\right)^{2}\right) \mathrm{d} \tau\right\} \mathrm{d} \tau= \\
& \frac{2}{t} \int_{0}^{t}\left(1-\frac{\tau}{t}\right)\left(R_{\eta^{2} \eta^{2}}(\tau)-\left(\sigma_{\eta}^{2}+\mu_{\eta}^{2}\right)^{2}\right) \mathrm{d} \tau,
\end{aligned}
$$

Eqs. (B.15) and (B.16) provide general expression for any stationary process. In the case of zero-mean Gaussian process (see Ochi (1990)), the expressions simplify to: 


$$
\begin{gathered}
\operatorname{Var}(\langle\eta\rangle)=\frac{2}{t} \int_{0}^{t}\left(1-\frac{\tau}{t}\right) R_{\eta \eta}(\tau) \mathrm{d} \tau \\
\operatorname{Var}\left(\left\langle\eta^{2}\right\rangle\right)=E\left[\left\langle\eta^{2}\right\rangle^{2}\right]-E^{2}\left[\left\langle\eta^{2}\right\rangle\right]=\frac{4}{t} \int_{0}^{t}\left(\left(1-\frac{\tau}{t}\right) R_{\eta \eta}^{2}(\tau)\right) \mathrm{d} \tau
\end{gathered}
$$

Consequently, the time length of the signal will be determined when the variance selected as indicator from Eqs. (B.17) or (B.18), reaches a certain minimum value. This procedure is expected to provide different time lengths of the recorded signal for the sea conditions tested, providing larger sampling times for test conditions that correspond to extreme sea states in the scatter diagram.

The procedure proposed above provides a proper methodology to determine the time duration of the recorded signal for sea conditions where the sea elevation is known, such as tests conducted in a wave basin. However, in full-scale applications only the measured motions of a ship or platform are available. The problem is therefore: Is this procedure appropriate when it is applied to the ship motions?

Let $\eta(t)$ be a realization of a certain sea condition in a time interval $[0, T]$. In order to properly represent the motions induced in a floating vessel by this sea condition, let be $\ell$ a sufficiently smooth function of the value $\eta$ of the sea condition under study. On the other hand, ships and platforms usually behave themselves as a low-pass filters. Therefore, the realization of the new process (which represents a certain response of the ship, such as the heave motion) can be obtained as:

$$
z_{i}(t)=\frac{1}{\Delta t} \int_{t}^{t+\Delta t} \ell(\eta(\tau)) \mathrm{d} \tau,
$$

where $t<T$ and $\Delta t$ is assumed sufficient to filter the high frequency component of the resulting process.

The expected value is estimated with Eq. (B.20), which is the result of making use of the definition of the expected value and the linearity of the operators involved in the calculation. 


$$
E[z(t)]=\frac{1}{\Delta t} \int_{t}^{t+\Delta t} E[\ell(\eta(\tau))] \mathrm{d} \tau
$$

where,

$$
E[\ell(\eta(\tau))]=\int_{-\infty}^{+\infty} \ell(\eta(\tau)) f(\eta ; \tau) d \eta
$$

in this equation $f$ stands for the probability density function of $\eta(\tau)$.

With regards to the autocorrelation function,

$$
R_{z z}\left(t_{1}, t_{2}\right)=E\left[z\left(t_{1}\right) z\left(t_{2}\right)\right]=\frac{1}{\Delta t_{1} \Delta t_{2}} \int_{t_{1}}^{t_{1}+\Delta t_{1}} \int_{t_{2}}^{t_{2}+\Delta t_{2}} E\left[\ell\left(\eta\left(\tau_{1}\right)\right) \ell\left(\eta\left(\tau_{2}\right)\right)\right] \mathrm{d} \tau_{1} \mathrm{~d} \tau_{2}
$$

where,

$$
E\left[\ell\left(\eta\left(\tau_{1}\right)\right) \ell\left(\eta\left(\tau_{2}\right)\right)\right]=\int_{-\infty}^{+\infty} \int_{-\infty}^{+\infty} \ell\left(\eta_{1}\right) \ell\left(\eta_{2}\right) f\left(\eta_{1}, \eta_{2} ; \tau_{1}, \tau_{2}\right) \mathrm{d} \eta_{1} \mathrm{~d} \eta_{2}
$$

More particularly,

$$
E\left[z^{2}(t)\right]=R_{z z}(t, t)=\frac{1}{\Delta t^{2}} \int_{0}^{t+\Delta t} \int_{0}^{t+\Delta t} E\left[\ell\left(\eta\left(\tau_{1}\right)\right) \ell\left(\eta\left(\tau_{2}\right)\right)\right] \mathrm{d} \tau_{1} \mathrm{~d} \tau_{2}
$$

Consequently, the variance is given by:

$$
\operatorname{Var}(z(t))=E\left[z^{2}(t)\right]-E^{2}[z(t)]=R_{z z}(t)-E\left[z^{2}(t)\right]
$$

It must be noted that, in general, even if the wave elevation is stationary, the induced motion does not have stationarity with respect to the autocorrelation function, but it is stationary with respect to the expected value. Moreover, the autocorrelation function, $R_{\eta \eta}(\tau)$, is equivalent to the power spectral density function, $S_{\eta \eta}(\omega, \mathrm{k})$, by virtue of the the Wiener-Kinchine theorem. Therefore, the resulting statistical properties of the motions spectra are not stationary.

From a practical point of view, however, in some cases the measured motions may 
provide a good input of the procedure proposed to select the optimal sample time. In fact, it is expected that the uncertainty introduced by the non-stationarity of the motions will be minimum in systems moored with a fixed heading relative to the seabed, because the induced non-stationarity is reduced to the RAOs variation in frequency-domain and the sea mean direction, limiting the possible wind and current effects on the heading of the platform and, as consequence, on the non-stationarity of the motions. As a preliminary evaluation of this procedure, the estimated sampling time will be obtained through the measured heave motion of the semisubmersible platform employed during the experimental campaign. However, due to the limitations of the tests carried out in the wave basin, full-scale applications of this method should be discussed more thoroughly with the analysis of real data of the measured motions. 


\section{APPENDIX C - STANDARD MODELS FOR WAVE SPECTRUM}

A detailed description of the wave spectrum models adopted in this work is provided in (BISHOP; PRICE, 1979) and (TORSETHAUGEN; HAVER, 2004). In the following the main formulae of the JONSWAP wave spectrum and the Torsethaugen wave spectrum are addressed.

\section{JONSWAP wave spectrum}

The JONSWAP wave spectrum was derived from the analysis of the data collected during the Joint North Sea Wave Observation Project (JONSWAP) and it can be considered an improvement of the PM wave spectrum. The expression for this type of wave spectrum is defined by,

$$
S_{\eta \eta}(f)=0.3125 H_{s}^{2} T_{p}^{2} f^{-5} \exp \left\{-1.25 T_{p}^{-4} f^{-4}\right\}(1-0.287 \ln \gamma) \gamma\left(\exp \left\{-0.5\left(\frac{T_{p} f-1}{\sigma}\right)^{2}\right\}\right),
$$

where the parameter, $\sigma$, is a measure of the width of the spectral peak and can be written as,

$$
\begin{cases}\sigma=0.07, & \text { for } T_{p} f<1 \\ \sigma=0.09, & \text { for } T_{p} f \geq 1,\end{cases}
$$

and $\gamma$ stands as the peakedness of the spectrum relative to a fully developed spectrum. As a matter of fact, when $\gamma$ is equal to one, JONSWAP spectrum equals to the PM spectrum. In this thesis the peak enhancement factor has been computed as proposed by Torsethaugen and Haver (2004): 


$$
\gamma=\max \left\{1,42.2\left(\frac{2 \pi H_{s}}{g T_{p}^{2}}\right)\right\}
$$

where $g$ is the gravity acceleration.

\section{Torsethaugen wave spectrum}

The Torsethaugen wave spectrum has been commonly used as a model for the double peak spectra at Norwegian seas. It allows the representation of the sea states as a combination of a locally wind generated sea and an incoming swell system. The simplified wave spectrum adopted in this thesis can be defined as a summation of two JONSWAP type wave spectra as follows:

$$
S_{\eta \eta}(f)=\sum_{j=1}^{2} E_{j} S_{j}(f)
$$

where $j=1$ represents the primary sea system (locally wind generated) and $j=2$ is the secondary sea system (swell). Regarding the values of $E$, they can be defined as,

$$
\begin{cases}E=\frac{1}{16} H_{1}^{2} T_{p 1}, & \text { for } j=1 \\ E=\frac{1}{16} H_{2}^{2} T_{p 2}, & \text { for } j=2,\end{cases}
$$

where $H_{1}, T_{p 1}, H_{2}$ and $T_{p 2}$ are given by Eqs. (C.10) and (C.11) for wind sea dominated cases while for swell dominated cases, Eqs. (C.13) and (C.14) are used to compute these parameters.

The non dimensional spectral density, $S_{j}$, can be written as,

$$
\left\{\begin{array}{l}
S_{\eta \eta}(f)=3.26 A_{\gamma} f_{1}^{-4} \exp \left\{f_{1}^{-4}\right\} \gamma\left(\exp \left\{-0.5\left(\frac{f_{1}-1}{\sigma}\right)^{2}\right\}\right), \text { for } j=1 \\
S_{\eta \eta}(f)=3.26 A_{\gamma} f_{2}^{-4} \exp \left\{f_{2}^{-4}\right\}, \quad \text { for } j=2,
\end{array}\right.
$$

The parameters included in Eq. (C.6) are defined as follows,

$$
\begin{aligned}
& f_{1}=f T_{p 1} \\
& f_{2}=f T_{p 2},
\end{aligned}
$$




$$
\begin{gathered}
A_{\gamma}=\frac{1+1.1(\ln (\gamma))^{1.19}}{\gamma}, \\
\left\{\begin{array}{l}
\sigma=0.07, \quad \text { for } T_{p} f<1 \\
\sigma=0.09, \quad \text { for } T_{p} f \geq 1,
\end{array}\right.
\end{gathered}
$$

Moreover, each sea state is then classified as swell dominated sea or wind dominated sea:

- Wind sea: $T_{p} \leq a_{f} H_{s}^{\frac{1}{3}}$

- Swell sea: $T_{p}>a_{f} H_{s}^{\frac{1}{3}}$

where $a_{f}$ is a constant, which value is given in Tab. C.1.

\section{Wind Sea Dominated Cases}

- Significant wave height for wind sea and swell are defined as,

$$
\begin{aligned}
& H_{1}=R_{w} H_{s} \\
& H_{2}=\left(1-R_{w}^{2}\right)^{0.5} \\
& R_{w}=\left(1-a_{10}\right) \exp \left\{-\left(\frac{\varepsilon_{l}}{a_{1}}\right)^{2}\right\}+a_{10},
\end{aligned}
$$

where $\varepsilon_{l}$ given by Eq. (C.16), $a_{1}$ and $a_{10}$ are constants as in Tab. C.1.

- Spectral peak periods are given by,

$$
\begin{aligned}
& T_{p 1}=T_{p} \\
& T_{p 2}=a_{f} H_{s}^{1 / 3}+b_{1},
\end{aligned}
$$

where $b_{1}$ is constant, which value is provided in Tab. C.1.

- Peak enhancement factor can be written as,

$$
\begin{aligned}
& \gamma=K_{g} s_{p}^{6 / 7} \\
& s_{p}=\frac{2 \pi H_{1}}{g T_{p 1}^{2}} .
\end{aligned}
$$

where $g$ is gravity acceleration and $k_{g}$ is constant as given in Tab. C.1. 


\section{Swell Sea Dominated Cases}

- Significant wave heights are defined as,

$$
\begin{aligned}
& H_{1}=R_{w} H_{s} \\
& H_{2}=\left(1-R_{w}^{2}\right)^{0.5} \\
& R_{w}=\left(1-a_{20}\right) \exp \left\{-\left(\frac{\varepsilon_{u}}{a_{2}}\right)^{2}\right\}+a_{20},
\end{aligned}
$$

where $\varepsilon_{u}$ given by Eq. (C.17), $a_{2}$ and $a_{20}$ are constants as in Tab. C.1.

- Spectral peak periods are given by,

$$
\begin{aligned}
& T_{p 1}=T_{p} \\
& T_{p 2}=a_{f} H_{2}^{1 / 3},
\end{aligned}
$$

where $b_{1}$ is constant, which value is provided in Tab. C.1.

- Peak enhancement factor is expressed as,

$$
\begin{aligned}
& \gamma=\gamma_{f}\left(1+a_{3} \varepsilon_{u}\right) \\
& \gamma_{f}=K_{g} s_{f}^{6 / 7} \\
& s_{p}=\frac{2 \pi H_{1}}{g\left(a_{f} H_{s}^{1 / 3}\right)^{2}} .
\end{aligned}
$$

$\varepsilon_{u}$ in Eqs. (C.14) and (C.15) is given by Eq. C.17. Regarding the constants $a_{2}, a_{3}, a_{20}$, their values are provided in Tab. C.1.

Non-dimensional scales for the spectral peak period found in the formulae provided above are defined as,

- For wind sea,

$$
\varepsilon_{l}=\frac{a_{f} H_{s}^{1 / 3}-T_{p}}{a_{f} H_{s}^{1 / 3}-a_{e} H_{s}^{0.5}},
$$

- For swell sea,

$$
\varepsilon_{u}=\frac{T_{p}-a_{f} H_{s}^{1 / 3}}{a_{u}-a_{f} H_{s}^{1 / 3}} .
$$


$a_{e}$ and $a_{u}$ can be found in Tab. C.1.

Table C.1: Empirical parameter values for Torsethaugen wave spectrum.

\begin{tabular}{cc} 
Parameter & Value \\
\hline$a_{f}$ & 6.6 \\
$a_{e}$ & 2 \\
$a_{u}$ & 25 \\
$a_{10}$ & 0.7 \\
$a_{1}$ & 0.5 \\
$a_{20}$ & 0.6 \\
$a_{2}$ & 0.3 \\
$a_{3}$ & 6 \\
$b_{1}$ & 2 \\
$k_{g}$ & 35 \\
\hline
\end{tabular}




\section{APPENDIX D - ANALYSIS OF THE HEAVE MOTION}

Through the Morison equation it is possible to estimate the forces acting on cylindrical shaped offshore structures. The computation of these forces allows the estimation of the motions of a semisubmersible platform (CHAKRABARTI, 2005). In this appendix, due to the particular interest the heave motions previously explained in section 3.3, only the vertical motion of a platform is characterized, along with a discussion regarding its most significant parameters. In order to provide an useful illustration of the heave motion features, the main characteristics of the platform provided in chapter 5 have been adopted to compute the relations shown herein.

In this appendix, the vertical responses of the platform are estimated assuming that the exciting forces are consequence of a wave traveling from port to starbord side of the platform. This approach allows to keep the model as simple as possible. Thus, using linear deep water theory and the Morison equation, the vertical force acting on the platform is obtained as the sum of the following terms,

- Columns: It has been assumed that the waves are traveling at a right angle of the stern-forward direction of the platform. Consequently, the relative phase of the wave to the port and the starbord sides of the platform is $\theta= \pm \frac{k B}{2}-\omega t$, being $B$ the total beam of the platform, $k$ the wave number and $\omega$ the wave frequency. Additionally, the hydrodynamic mass term of the columns has been assumed sufficiently small to be neglected. Therefore, the force acting in each column can be written as follows,

$$
F_{z}^{c}=P_{z=-h_{c}} A_{c o l}=\rho g \zeta_{a} A_{c} \exp \left\{-k h_{c o l}\right\} \cos (\theta),
$$


where $A_{\text {col }}$ stands for the area of the column (adopted as a constant along the height of the column), $h_{c o l}$ is the height of the columns.

- Pontoon sections (port or starbord sides): For these sections of the pontoon the phase properties are equal to the ones used to estimate the force acting on the columns. Thus,

$$
F_{z}^{p s}=-C_{m} \rho \nabla_{P_{p s}} \zeta_{a} \omega^{2} \exp \left\{-k\left(h_{c o l}+h_{\text {pont }}\right)\right\} \cos (\theta),
$$

with $C_{m}=1+C_{a}$ and $\nabla_{P_{p s}}=A_{p} L$ the displacement of each part of the pontoon under study.

- Pontoon section (forward or stern): The procedure followed to estimate the force acting on these parts of the pontoon is similar to the one adopted above, but in this case the phase of the force acting on each longitudinal element of the pontoon is given by $\theta=k x$, with $x \in[-B / 2, B / 2]$. consequently, the force acting on one part of the pontoon can be computed as,

$$
\begin{aligned}
F_{z}^{f s}= & -\int_{-B / 2}^{B / 2} C_{m} \rho A_{p} \zeta_{a} \omega^{2} \exp \left\{-k\left(h_{\text {col }}+h_{\text {pont }}\right)\right\} \cos (k x-\omega t) \mathrm{d} x= \\
& -\frac{2}{k} C_{m} \rho A_{p} \zeta_{a} \omega^{2} \exp \left\{-k\left(h_{\text {col }}+h_{\text {pont }}\right)\right\} \sin \left(\frac{k B}{2}\right) \cos (\omega t) .
\end{aligned}
$$

The vertical force acting on the whole structure can be estimated as the sum of the respective forces listed above, 


$$
\begin{aligned}
F_{z}= & \rho g \zeta_{a} A_{c o l}\left(\exp \left\{-k h_{c o l}\right\}-C_{m} \frac{\nabla_{P_{p s}}}{\nabla_{c t}} k h_{c o l} \exp \left\{-k\left(h_{c o l}+h_{\text {pont }}\right)\right\}\right) \times \\
& \left(\cos \left(\frac{k B}{2}-\omega t\right)+\cos \left(\frac{k B}{2}-\omega t\right)\right)-\frac{4}{k B} C_{m} \rho \nabla_{P_{f s}} \zeta_{a} \omega^{2} \exp \left\{-k\left(h_{c o l}+h_{\text {pont }}\right)\right\} \sin \left(\frac{k B}{2}\right) \cos (\omega t) \\
= & \rho g \zeta_{a} A_{c o l}\left[\left(\exp \left\{-k h_{c o l}\right\}-C_{m} \frac{\nabla_{P_{p s}}}{\nabla_{c t}} k h_{c o l} \exp \left\{-k\left(h_{c o l}+h_{\text {pont }}\right)\right\}\right) \cos \left(\frac{k B}{2}\right)\right. \\
& \left.-\frac{4}{B} C_{m} \frac{\nabla_{P_{f s}}}{\nabla_{c t}} h_{c o l} \exp \left\{-k\left(h_{c o l}+h_{\text {pont }}\right)\right\} \sin \left(\frac{k B}{2}\right)\right] \cos (\omega t) .
\end{aligned}
$$

Now, the total vertical force can be normalized by the force defined by the product of the total water plane area, $A_{\mathrm{col}_{T}}$, and $\rho g \zeta_{a}$,

$$
\begin{aligned}
F_{z}^{*}= & {\left[\left(\exp \left\{-k h_{c o l}\right\}-C_{m} \frac{\nabla_{P_{p s}}}{\nabla_{c t}} k h_{c o l} \exp \left\{-k\left(h_{c o l}+h_{\text {pont }}\right)\right\}\right)^{2}\right.} \\
& \left.+\left(\frac{4}{B} C_{m} \frac{\nabla_{P_{f s}}}{\nabla_{c t}} h_{c o l} \exp \left\{-k\left(h_{c o l}+h_{\text {pont }}\right)\right\}\right)^{2}\right]^{\frac{1}{2}} \cos \left(\frac{k B}{2}-\alpha\right) \cos (\omega t),
\end{aligned}
$$

where

$$
\tan (\alpha)=\frac{-\frac{4}{B} C_{m} \frac{\nabla_{P_{f s}}}{\nabla_{c t}} h_{c o l} \exp \left\{-k\left(h_{c o l}+h_{\text {pont }}\right)\right\}}{\exp \left\{-k h_{c o l}\right\}-C_{m} \frac{\nabla_{P_{p s}}}{\nabla_{c t}} k h_{c o l} \exp \left\{-k\left(h_{c o l}+h_{\text {pont }}\right)\right\}} .
$$

From the total vertical force provided in Eq. (D.6), the transfer function of the undamped heave motion is given by,

$$
\left|Z_{z}\right|=\frac{2 \rho g A_{\text {col }_{T}} F_{z}^{*}}{c_{z z}\left(1-\left(\frac{\omega}{\omega_{n}}\right)^{2}\right)},
$$


where the restoring coefficient , $c_{z z}$, follows from the hydrostatic buoyancy force of the columns,

$$
c_{z z}=2 \rho g A_{c o l_{T}}
$$

On the other hand, the total mass of the system is given by the mass of the floating platform and the hydrodynamic mass of the pontoon,

$$
\rho \nabla+m_{z z}=\rho\left(2 \nabla_{c t}+C_{m} \nabla_{\text {pont }}\right)
$$

Consequently, the natural frequency of this structure can be estimated as,

$$
\omega_{n}=\left(\frac{g A_{c o l}}{2 \nabla_{c t}+C_{m} \nabla_{p o n t}}\right)^{\frac{1}{2}}
$$

The relations provided until now are useful to predict the undamped motion of the semisubmersible platform under study. As a matter of fact, the heave transfer function presents a cancellation point close to the resonance and its value at the resonant frequency is infinite. The effects of the velocity-dependent viscous forces can be introduced through the linearized form of the drag component of the Morison equation,

$$
F_{z}^{D_{L}}=\frac{4}{3 \pi} \rho C_{d} A_{p}[\bar{\omega}-\dot{z}](\bar{\omega}-\dot{z})
$$

where $\bar{\omega}$ is the vertical orbital velocity, $\dot{z}$ is the vertical velocity of the platform and $A_{p}$ is the effective area of the pontoon. Therefore, and knowing that $[\bar{\omega}-\dot{z}]$ is the representative velocity amplitude, the drag force can be computed in terms of the heave transfer function,

$$
F_{z}^{D_{L}}=\frac{4}{3 \pi} \rho C_{d} A_{p}\left[\exp \left\{-k\left(h_{c o l}+h_{\text {pont }}\right)\right\}-\left|Z_{z}\right| e^{i \phi}\right] \zeta_{a} \omega A_{p}(\bar{\omega}-\dot{z})=b_{33}(\bar{\omega}-\dot{z})
$$


The resulting terms of the velocity-dependent drag force, $b_{z z} \bar{\omega}$, and damping force, $b_{z z} \dot{z}$, can be used to estimate the magnitude of the transfer function of the heave motion, leading to,

$$
\left|Z_{z}\right|=\frac{F_{z}+b_{33} \bar{\omega}}{c_{z z} \zeta_{a}\left(\left(1-\left(\frac{\omega}{\omega_{n}}\right)^{2}\right)^{2}+\left(\frac{b_{z z} \omega}{c_{z z}}\right)^{2}\right)^{\frac{1}{2}}}
$$

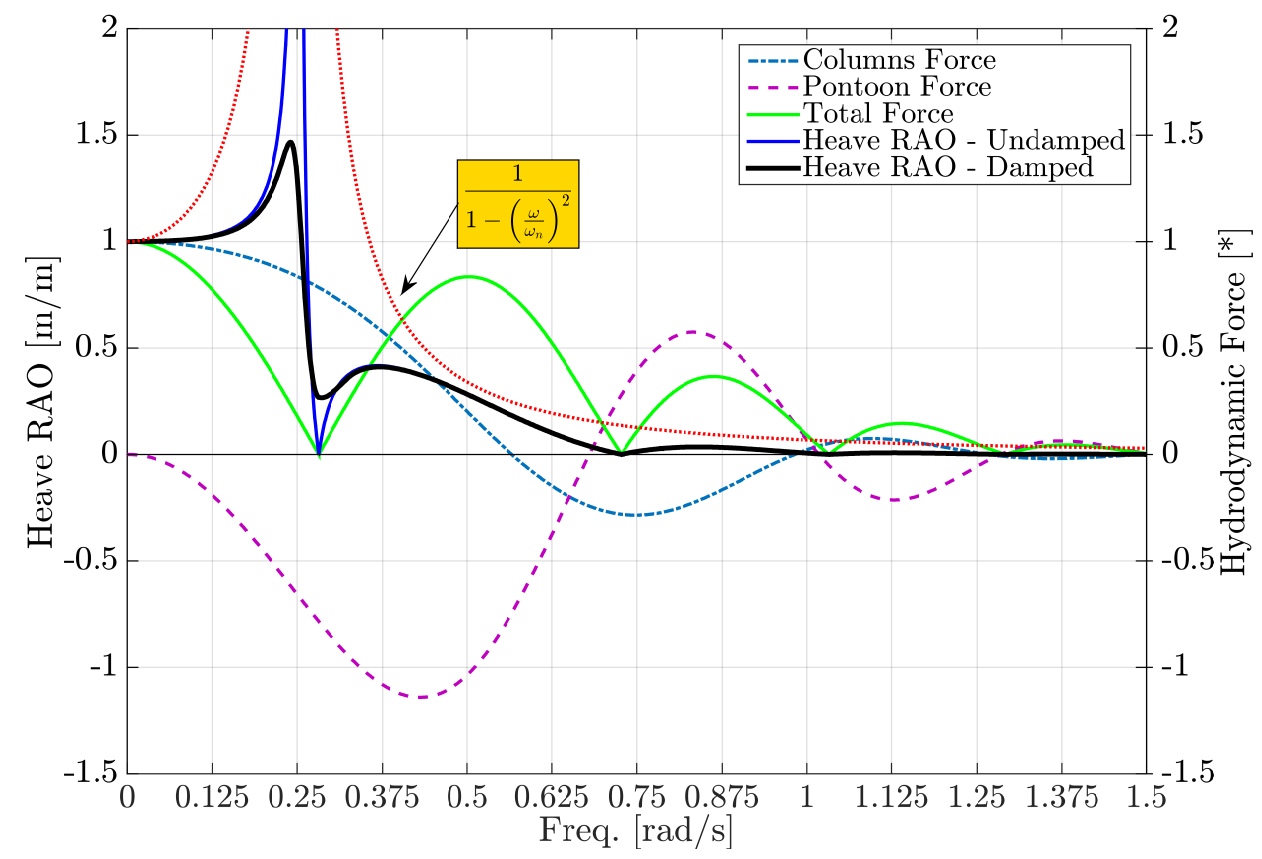

Figure D.1: Illustration of heave RAO of motion.

Equation D.13 provides the transfer function of the heave motion, including the drag and damping forces. Since the value of the velocity-dependent drag force cannot be computed until the velocity of the structure is known, this method to compute the heave motion RAO results in an iterative process. It is convenient to highlight that the resulting transfer function is characterized by:

- As it has been shown before, the inertia forces vanishes close to the resonance of the heave motion. Consequently, the vertical motion of the structure depends exclusively of the velocity-dependent drag forces. Thus, the amplitude at the (former) cancellation point close to the resonance of the motion can be estimated as, 


$$
\left|Z_{z}\right|=\frac{b_{33} \bar{\omega}}{c_{z z}\left(1-\left(\frac{\omega}{\omega_{n}}\right)^{2}\right)}
$$

- Regarding the amplitude of the motion at the resonance frequency, the first term of the denominator is reduced to zero (leading to infinite amplitude of the motions if only the inertia forces are computed), leaving only the damping term. Consequently, the amplitude of the motion at the resonant frequency is given by,

$$
\left|Z_{z}\right|=\frac{F_{z}+b_{33} \bar{\omega}}{\zeta_{a}\left(b_{z z} \bar{\omega}\right)} .
$$

The features described above, with regards to the heave motion transfer function, are also shown in Fig. D.1. This figure provides the RAO of the heave motion estimated through the relations deduced in this appendix and using the characteristics of the platform provided in chapter 5 . This figure also reflects other characteristics previously listed in section 3.3 regarding Fig. 3.3, such as the fact that in short waves the motion of the platform is minimum and in long waves the platform predictably follows the wave amplitude. The estimated heave RAO also shows that the resonant frequency is equal to $\omega_{n}=0.262 \mathrm{rad} / \mathrm{s}$, that is close to frequency of the vertical (inertia) forces cancellation $\left(\omega_{c}=0.281 \mathrm{rad} / \mathrm{s}\right)$. In the high frequency range, that corresponds to short wave periods, the magnitude of the transfer function indicates successively smaller oscillation amplitudes, with multiple cancellation points due to the phase of the force and the small influence of the velocity-dependent forces. Finally, the analytical approach adopted in this appendix to deduce the heave RAO can be intended as a reasoned explanation of the influence that non-linear viscous forces will have on the heave motion near the resonance, modifying the linearly estimated responses as well as the cancellation point close to the resonance as shown in section 3.3. 


\section{APPENDIX E - CONSTRUCTION OF A SMOOTH PRIOR DISTRIBUTION}

In section 4.4.1 it has been shown that the hypothesis that the unknown spectrum should be smooth can be expressed through a matrix that stands as an approximation of the second order finite difference. Thus, the prior distribution is given by (for a given direction or frequency),

$$
p(\mathrm{x}) \propto \exp \left\{-\frac{\|\mathbf{H x}\|^{2}}{2 \sigma^{2}}\right\},
$$

where $\mathbf{H}$ can be defined for a given frequency $(m)$ as (following a similar procedure that in section 4.4.1),

$$
H=\left[\begin{array}{cccccccc}
1 & -2 & 1 & 0 & \cdots & 0 & 0 & 0 \\
0 & 1 & -2 & 1 & \cdots & 0 & 0 & 0 \\
\vdots & \vdots & \vdots & \vdots & \ddots & \vdots & \vdots & \vdots \\
0 & 0 & 0 & 0 & \cdots & 1 & -2 & 1
\end{array}\right] \in \mathbb{R}^{(K-1) \times(K+1)}
$$

where $K$ is the number of discrete wave heading considered.

Nevertheless, due to the rank of matrix $H$ (that is $K-1$ ) boundary conditions should be imposed. In the direction domain (NIELSEN, 2005) it is assumed that: $S_{\eta \eta}\left(\omega_{m}, \beta_{0}\right)=$ $S_{\eta \eta}\left(\omega_{m}, \beta_{K}\right)$ and $S_{\eta \eta}\left(\omega_{m}, \beta_{K+1}\right)=S_{\eta \eta}\left(\omega_{m}, \beta_{1}\right)$. These conditions leads to the following matrix $\mathbf{H}_{1}$, 


$$
\mathbf{H}_{1}=\left[\begin{array}{cccccccc}
-2 & 1 & 0 & 0 & \cdots & 0 & 0 & 1 \\
1 & -2 & 1 & 0 & \cdots & 0 & 0 & 0 \\
\vdots & \vdots & \vdots & \vdots & \ddots & \vdots & \vdots & \vdots \\
1 & 0 & 0 & 0 & \cdots & 0 & 1 & -2
\end{array}\right] \in \mathbb{R}^{(K+1) \times(K+1)}
$$

When it comes to the frequency-domain, for a given direction $k$, the hypothesis is that $S$ is smooth and close to zero in the boundary. Thus,

$$
\left.S_{\eta \eta}\left(\omega_{0}, \beta_{k}\right)=\frac{1}{2}\left(S_{\eta \eta}\left(\omega_{0-1}, \beta_{k}\right)+S_{\eta \eta}\left(\omega_{0+1}, \beta_{k}\right)\right)+\varepsilon_{p_{0}}=\frac{1}{2}\left(S_{\eta \eta}\left(\omega_{0+1}, \beta_{k}\right)\right)\right)+\varepsilon_{p_{0}}, \quad \varepsilon_{p_{0}} \sim \mathcal{N}\left(0, \sigma_{p}^{2}\right),
$$

$$
\left.S_{\eta \eta}\left(\omega_{M}, \beta_{k}\right)=\frac{1}{2}\left(S_{\eta \eta}\left(\omega_{M-1}, \beta_{k}\right)+S_{\eta \eta}\left(\omega_{M+1}, \beta_{k}\right)\right)+\varepsilon_{p_{M}}=\frac{1}{2}\left(S_{\eta \eta}\left(\omega_{M-1}, \beta_{k}\right)\right)\right)+\varepsilon_{p_{M}}, \quad \varepsilon_{p_{M}} \sim \mathcal{N}\left(0, \sigma_{p}^{2}\right),
$$

It should be highlighted that the wave spectrum has been extended by 0 outside of the frequency-domain since the prior believe says that is smooth. Finally, the resulting $\mathbf{H}_{2}$ is,

$$
\mathbf{H}_{2}=\left[\begin{array}{cccccccc}
2 & -1 & 0 & 0 & \cdots & 0 & 0 & 0 \\
1 & -2 & 1 & 0 & \cdots & 0 & 0 & 0 \\
\vdots & \vdots & \vdots & \vdots & \ddots & \vdots & \vdots & \vdots \\
0 & 0 & 0 & 0 & \cdots & 0 & -1 & 2
\end{array}\right] \in \mathbb{R}^{(M+1) \times(M+1)}
$$

However, since the limits of the frequency-domain are imposed by the limitation due to the frequency insensitivity of the sensor (in this case the semisubmersible platform), there is no reason to believe that $S$ must be zero at the boundary. Since the real values of the spectrum at the boundary in the frequency-domain are unknown, they can be assumed Gaussian random variables, that properly adhere to the Bayesian nature of the problem, such as,

$$
\left.S_{\eta \eta}\left(\omega_{0}, \beta_{k}\right) \sim \mathcal{N}\left(0, \frac{\sigma^{2}}{\lambda_{0}^{2}}\right) \quad \text { and } \quad S_{\eta \eta}\left(\omega_{M}, \beta_{k}\right)\right) \sim \mathcal{N}\left(0, \frac{\sigma^{2}}{\lambda_{M}^{2}}\right)
$$


Therefore, the prior distribution in the frequency-domain (in direction $k$ ) is given by,

$$
P(x) \propto \exp \left\{-\frac{\left\|\mathbf{H}_{2_{n}} \mathbf{x}\right\|^{2}}{2 \sigma^{2}}\right\},
$$

where $\mathbf{H}_{2_{n}}$ is,

$$
\mathbf{H}_{2_{n}}=\left[\begin{array}{cccccccc}
\lambda_{0} & 0 & 0 & 0 & \cdots & 0 & 0 & 0 \\
1 & -2 & 1 & 0 & \cdots & 0 & 0 & 0 \\
\vdots & \vdots & \vdots & \vdots & \ddots & \vdots & \vdots & \vdots \\
0 & 0 & 0 & 0 & \cdots & 0 & 0 & \lambda_{M}
\end{array}\right] \in \mathbb{R}^{(M+1) \times(M+1)}
$$

The value of the variance in each component of $S$ should be computed in order to estimate the values of $\lambda_{0}^{2}$ and $\lambda_{M}^{2}$. From Eq. (4.38) it is already known that the variance of each element of the sea spectrum can be expressed as,

$$
\operatorname{Var}\left(S_{j}\right)=\mathbf{e}_{j}\left(\int_{\mathbb{R}^{M+1}} S^{2} P(S) \mathrm{d} S\right) \mathbf{e}_{j}^{T}=\sigma^{2} \mathbf{e}_{j}\left(\mathbf{H}_{2_{n}}^{T} \mathbf{H}_{2_{n}}\right)^{-1} \mathbf{e}_{j},
$$

where $\mathbf{e}_{j}$ stands for the $j^{\text {th }}$ canonical basis vector in $\mathbb{R}^{M+1}$.

If the relation in Eq. (E.10) is computed using the matrix obtained in Eq. (E.6), one may realize that the maximum uncertainty is reached at the middle of the domain, since it is the furthest point from the boundary. Consequently, in order to make all the values equally uncertain, the following relation must be set:

$$
\operatorname{Var}\left(S_{M / 2}\right)=\operatorname{Var}\left(S_{0}\right)=\operatorname{Var}\left(S_{M}\right)=\sigma^{2} \mathbf{e}_{M / 2}\left(\mathbf{H}_{2_{n}}^{T} \mathbf{H}_{2_{n}}\right)^{-1} \mathbf{e}_{M / 2}
$$

This relation leads to,

$$
\lambda_{0}^{2}=\lambda_{M}^{2}=\frac{1}{\mathbf{e}_{M / 2}\left(\mathbf{H}_{2_{n}}^{T} \mathbf{H}_{2_{n}}\right)^{-1} \mathbf{e}_{M / 2}} .
$$

However, Eq. (E.13) requires to solve a non-linear equation, since $\mathbf{H}_{2_{n}}$ depends on $\lambda_{0}^{2}$ and $\lambda_{M}^{2}$. Thus, in order to simplify the computing process, Eq. (E.13) may be simplified as, 


$$
\lambda_{0}^{2}=\lambda_{M}^{2}=\frac{1}{\mathbf{e}_{M / 2}\left(\mathbf{H}_{2}^{T} \mathbf{H}_{2}\right)^{-1} \mathbf{e}_{M / 2}} .
$$

This modified boundary condition introduces slight changes on the original prior distribution (discussed in section 4.4.1). These modifications may improve the tendency of the method of locating spurious energy peaks at the bow and stern of the vessel in beam sea conditions, increasing the chances of determining these energy peaks elsewhere. Since the data used in this thesis does not reflect the drawback identified by Bispo et al. (2016), the evaluation of the effect resulting from this modification is something that has been left as a future work. 


\section{APPENDIX F - DRAG EFFECTS ARISING FROM MOORING LINES AND RISERS}

The semisubmersible platform, its risers and mooring lines make up a global system that has a complex dynamic response when excited by waves. In fact, the dynamics of a semisubmersible platform is quite different from the one presented by ship-shaped vessel commonly used to make wave inference, such as a FPSO (BISPO et al., 2016). Among other factors, an important part of this difference is related to the fact that the semisubmersible's motions are only weakly damped, one reason for this being the fact that radiated waves are small due large draft and the cylindrical shape of the columns. For this reason, the dynamics of semisubmersibles is known to be more sensitive to damping effects arising from mooring lines and risers (see, for instance Wijaya (2009)). Moreover, the results presented ins ection 5.2.3 suggest that a proper study of the influence of the damping and stiffness induced by risers and mooring lines in the heave RAO is needed to assess the accuracy of the method for estimating extreme waves with very high peak periods (typical above 20s), which will necessarily impose a significant resonant response of the heave motion of the Asgard-B platform.

The relevance of a more refined analysis of the motions in high wave periods becomes even stronger for future wave estimations based on a real semisubmersible platform. For this purpose a sensitive analysis has been carried out by means of time-domain analysis in ANSYS-AQWA, which provides a dedicated resource for the motion analysis of floating platform as well as the effects arising from the submerged lines (see, for instance Jeon et al. (2013)). Accordingly, the resulting model (see Fig. F.1) was composed by the 
hull of the platform, which was attached to 16 spread mooring lines and a flexible riser system of altogether 37 risers, including: Simple catenary risers, lazy wave and lazy-S risers and whose main features are provided in (LIAN, 1997). The riser and mooring lines are modelled in the software as a Morsion elements, while its structural properties and responses are modelled using the ANSYS-MECHANIAL APDL module (see (ANSYS, 2013)). Although the software controls the dynamics of the risers and mooring lines, some coefficients, such as added mass and drag, as well as the main inertia properties of the submerged lines must be provided. For this purpose, the values of the drag and added mass coefficient specified in (SARPKAYA, 2010) have been adopted, meanwhile the data detailed in (LIAN, 1997) are adopted to model the mass and inertia properties of the submerged lines.

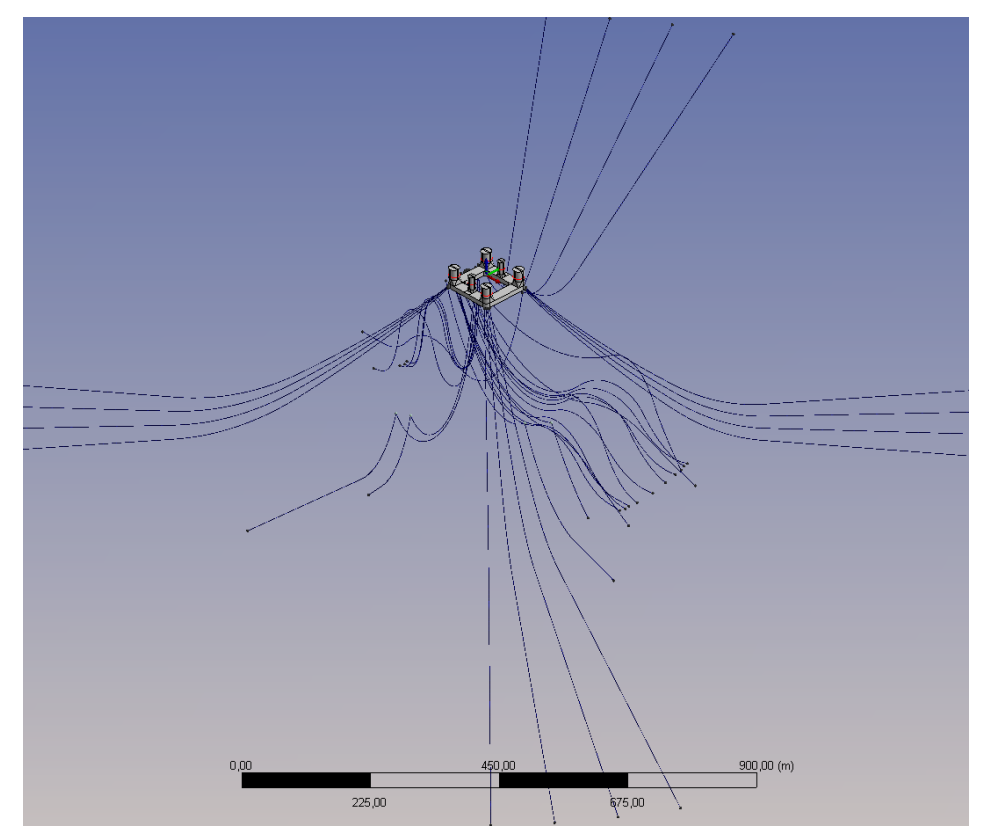

Figure F.1: ANSYS-AQWA model including the submerged lines.

Therefore, mooring and riser systems were included in the analysis in order to reproduce both the damping and stiffness effects. Figure F.2 shows the amplitude of the heave motion, $z$, along the time-domain. This result corresponds to the test carried out with ANSYS-AQWA including risers and mooring lines, with a regular wave with a steepness equal to $2 \%$. The response of the heave motion has been normalized by the wave amplitude, $A$. In the starting region $(T<400 \mathrm{~s})$ the mean amplitude of the heave response fluctuated significantly, time that seems to be related with the time span needed to reach the force equilibrium and, as a consequence, the steady state. Concerning the responses 
estimated for $T>400$ s (referent to the steady state), the mean value of the response amplitude remained approximately constant.

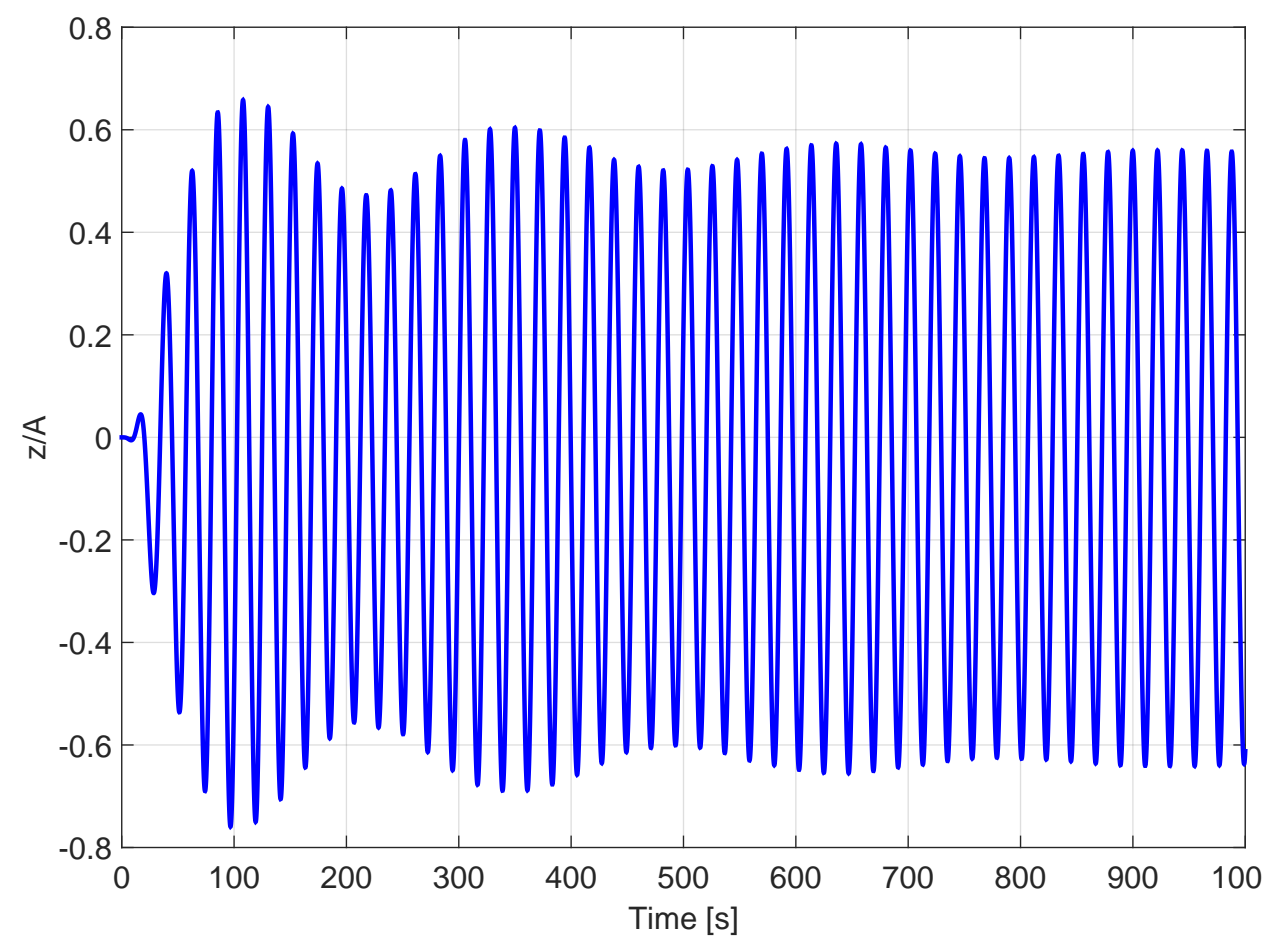

Figure F.2: Normalized heave amplitude, for the test with wave steepness equal to $2 \%$ and including the risers and mooring lines.

The RAO resulting from taking into account the submerged lines is included in Fig. F.3 for two different wave steepnesses: $0.64 \%$ and $2 \%$ (orange rhombus and magenta squares, respectively) along with the RAO obtained previously for the same wave steepness in Fig. 5.6 (magenta rhombus and orange squares, respectively). One may realize that the results in this figure including the submerged lines were carried out only for periods up to $24 \mathrm{~s}$, this is due to the lack of convergence of the simulations for higher periods.

The RAOs presented in Fig. F.3 show that the expected response of the heave motion, including risers and mooring effects in the numerical analysis, present narrow differences with the RAOs obtained taking in account only the drag effects acting on the pontoon. Therefore, this preliminary assessment indicates that no significant differences in the estimations of the sea condition should be expected if this new RAO is adopted. Nonetheless, a dedicated study (experimental and/or numerical) must be carried out in order to accurately determine the possible effects that submerged lines as well as environmental effects (i.e. wind \& currents) may have on the heave responses close to the resonance when deal- 


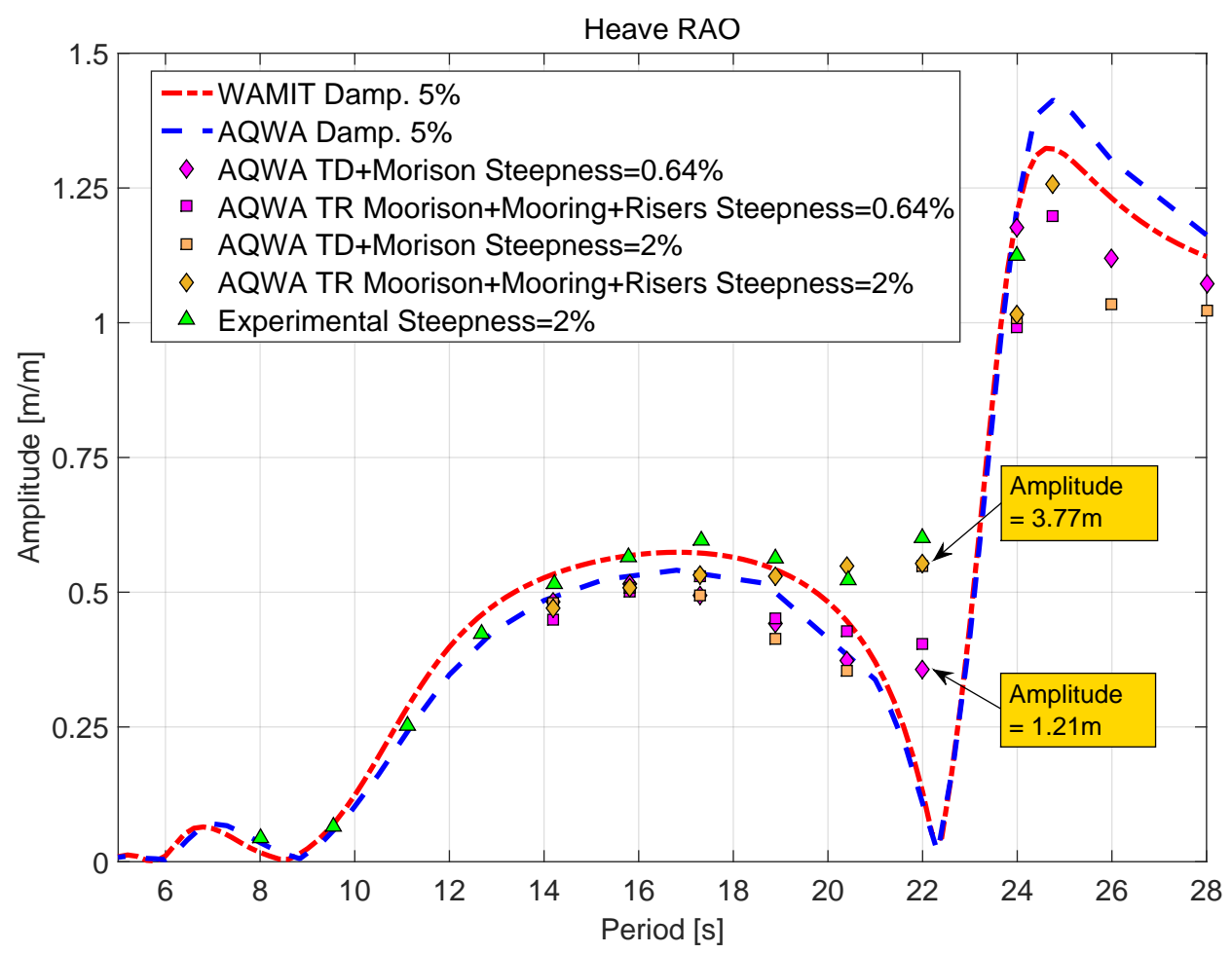

Figure F.3: RAO heave, including the drag effects due to the submerged lines.

ing with the motions recorded aboard a full-scale semisubmersible platform and, thus, avoid unnecessary accuracy losses in the estimation of extreme wave conditions. 